\title{
Faseroptische Gemischbildungsanalyse in Otto-Motoren bei direkteinspritzenden Brennverfahren
}

\author{
Dissertation \\ zur Erlangung des Doktorgrades \\ der Mathematisch-Naturwissenschaftlichen Fakultäten \\ der Georg-August-Universität zu Göttingen
}

vorgelegt von

Olaf Thiele

aus Hannover

Göttingen, 2004 
D7

Referent: Prof. Dr. W. Lauterborn

Korreferent: Prof. Dr. G. Marowsky

Tag der mündlichen Prüfung: 26.10.2004 


\section{Danksagung}

Die vorliegende Arbeit entstand am Laser-Laboratorium Göttingen e.V. Ich möchte mich herzlich bei allen Leuten bedanken, die zum Gelingen meiner Arbeit beigetragen haben.

Herrn Prof. Dr. Werner Lauterborn danke ich für Übernahme und Betreuung meiner Arbeit als Hauptreferent.

Danken möchte ich Herrn Prof. Dr. Gerd Marowsky für die Möglichkeit eine externe Dissertation am Laser Laboratorium Göttingen e.V. durchführen zu können und die vielen hilfreichen Anregungen.

Mein besonderer Dank gilt Herrn Volker Beushausen, dem Leiter der Arbeitsgruppe Verbrennungs- und Strömungsdiagnostik am Laser-Laboratorium Göttingen. Er stand mir immer mit kompetenten Ratschlägen zur Seite und trug in zahlreichen wissenschaftlichen Diskussionen entscheidend zum Erfolg dieser Arbeit bei.

Ebenso möchte ich Dr. Stefan Arndt, Dr. Roman Grzeszik und Dr. Jürgen Reimann für die hervorragende und sehr angenehme Zusammenarbeit im Rahmen der Messkampagne bei der Robert Bosch GmbH danken.

Mein Dank gebührt auch Herrn Dr. Werner Hentschel und Felix Kallmeyer von der Volkswagen AG, die mir als Ansprechpartner in technischen Fragen zur Seite standen und die Betreuung während der Messkampagne in Wolfsburg übernahmen.

Danken möchte ich auch allen meinen Kollegen am Laser-Laboratorium Göttingen für die freundschaftliche Zusammenarbeit. Unsere anregenden Diskussionen führten häufig zu neuen Denkanstößen und sorgten für viel Spaß bei der Arbeit. Dr. Thomas Müller und Thorsten Wesker standen mir bei einigen Experimenten hilfreich zur Seite. Auch den Kollegen Karsten Roetmann, Dr. Jochen Scholz und Alexander Grosch möchte ich für die Unterstützung und Zusammenarbeit danken.

Zuletzt und als erstes gilt mein besonderer Dank meiner Frau Marion, die mit viel Geduld und Liebe mir immer Kraft und Ansporn gab. 



\section{Inhaltsverzeichnis}

1. Einleitung 1

1.1. Motivation . . . . . . . . . . . . . . . . 1

1.2. Überblick . . . . . . . . . . . . . . . . . . . . . . . 3

2. Einführung 5

2.1. Ottomotorische Brennverfahren . . . . . . . . . . . . . 5

2.2. Lichtwellenleiter . . . . . . . . . . . . . . . . . . . . 9

3. Theoretische Grundlagen der angewandten Messverfahren 11

3.1. Raman-Spektroskopie . . . . . . . . . . . . . . . . . . . . . 11

3.1.1. Theoretische Grundlagen . . . . . . . . . . . . . . . 12

3.1.2. Lambda-Wert-Bestimmung . . . . . . . . . . . . . . . . . . . . 16

3.2. Laserinduzierte Fluoreszenz . . . . . . . . . . . . . . . . . . . . . . 18

3.2.1. Theoretische Grundlagen . . . . . . . . . . . . . . . . 18

3.2.2. Lambda-Wert-Bestimmung . . . . . . . . . . . . . . . . . . 20

3.3. Absorptionsspektroskopie . . . . . . . . . . . . . . . . . . 23

3.3.1. Theoretische Grundlagen . . . . . . . . . . . . . . . 23

3.3.2. Linienstärke und -profil von Absorptionslinien . . . . . . . . . . . 24

3.3.2.1. Natürliche Linienbreite . . . . . . . . . . . . . . . . . 25

3.3.2.2. Dopplerverbreiterung . . . . . . . . . . . . . . . 25

3.3.2.3. Druckverbreiterung . . . . . . . . . . . . . 26

3.3.3. Lambda-Wert-Bestimmung . . . . . . . . . . . . . . . . 27

4. Raman-spektroskopische Gemischbildungsanalyse in Ottomotoren 29

4.1. Komponenten des Detektionssystems . . . . . . . . . . . . . . . 30

4.1.1. Lasersystem . . . . . . . . . . . . . . . . . . . . . . 30

4.1.2. Signaldetektion . . . . . . . . . . . . . . 34

4.2. Voruntersuchungen . . . . . . . . . . . . . . . . . 35

4.2.1. Auswahl des Kraftstoffes . . . . . . . . . . . . . . . . . . . 36

4.2.2. Lambda-Wert-Bestimmung in homogenen Gemischen . . . . . . . . 38

4.2.3. Sprayuntersuchungen . . . . . . . . . . . . . . . . . . . . . 39

4.2.4. Eliminierung nichtlinearer Effekte durch Absorberfarbstoff . . . . . 41

4.2.5. Resonatoreigenschaften von Flüssigkeitströpfchen . . . . . . . . . . 45 
4.3. Motorische Untersuchungen . . . . . . . . . . . . . . . 50

4.3.1. Experimenteller Aufbau am Transparentmotor . . . . . . . . . . . 50

4.3.2. Ergebnisse der Freistrahldetektion . . . . . . . . . . . . . 53

4.3.2.1. Raman-spektroskopische $\lambda$-Wert-Bestimmung . . . . . . . 56

4.3.2.2. Zusammenhang zwischen Raman- und LIF-Messungen . . 61

4.4. Faseroptisches Sensorsystem . . . . . . . . . . . . . . . 63

4.4.1. Eigenschaften von Glasfasern bei hoher Laserleistung . . . . . . . . 63

4.4.2. Aufbau des faseroptischen Raman-Sensors . . . . . . . . . . . . . . 66

4.4.3. Motorische Untersuchungen . . . . . . . . . . . . . . . . 67

4.5. Neue Detektionskonzepte . . . . . . . . . . . . . . . . . . . 70

4.5.1. Sensorgeometrie . . . . . . . . . . . . . . . . 70

4.5.2. Spektrale Filterung der Raman-Signale . . . . . . . . . . . . . . . . 71

4.5.3. Vergleich unterschiedlicher Detektoren . . . . . . . . . . . . . 74

4.5.4. Erste Untersuchungen von Gemischzusammensetzungen . . . . . . . 81

4.6. Zusammenfassung und Ausblick . . . . . . . . . . . . . . . . 82

5. Gemischbildungsanalyse mittels Absorptionsspektroskopie 85

5.1. Kraftstoffdichtebestimmung mittels Absorptionsspektroskopie . . . . . . . 86

5.1.1. Experimenteller Aufbau . . . . . . . . . . . . . . . 86

5.1.2. Messungen unter motorischen Bedingungen . . . . . . . . . . . 88

5.1.3. Näherungsverfahren zur $\lambda$-Wert-Bestimmung . . . . . . . . . . . . 91

5.1.4. Untersuchungen am Serienmotor . . . . . . . . . . . . . 92

5.1.5. Fehlerbetrachtung . . . . . . . . . . . . . . 97

5.1.6. Temperatureinfluss auf IR-Absorption . . . . . . . . . . . . . 100

5.2. Spektroskopische Bestimmung der Sauerstoffdichte . . . . . . . . . . . . . . 104

5.2.1. VCSEL-Laser . . . . . . . . . . . . . . . . . . . . . . 104

5.2.2. Signaldetektion . . . . . . . . . . . . . . . . 105

5.2.3. Experimenteller Aufbau . . . . . . . . . . . . . . 106

5.2.4. Druckvariation . . . . . . . . . . . . . . . . 107

5.3. Zusammenfassung und Ausblick . . . . . . . . . . . . . . . . 111

$\begin{array}{ll}\text { Literaturverzeichnis } & 113\end{array}$

A. Anhang 


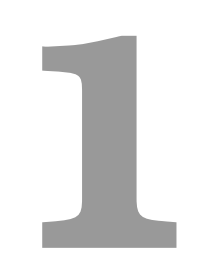

\section{Einleitung}

\subsection{Motivation}

Dem Wunsch der modernen Gesellschaft nach individueller Mobilität stehen die damit verbundenen schädlichen Einflüsse auf die Umwelt gegenüber. Ein schonender Umgang mit den vorhandenen Ressourcen und neue umweltverträglichere Konzepte sind für moderne Motorentechniken daher unumgänglich. Da nach wie vor fossile Brennstoffe als Hauptenergieträger in Kraftfahrzeugen eingesetzt werden, müssen Wege gefunden werden, die Emission klima-, umwelt- und gesundheitsschädigender Abgase zu minimieren. Zwar verringerte sich seit 1985 mit der Einführung des 3-Wege-Katalysators mit $\lambda$-Regelung in der Europäischen Union der Ausstoß verkehrsbedingter Schadstoffe bereits erheblich, doch auch ein geringerer Kraftstoffverbrauch ist notwendig, um die Schadstoffemission trotz eines stetig zunehmenden Individualverkehrs weiter senken zu können.

Bei modernen Ottomotoren setzen sich zur Steigerung der Effizienz der motorischen Verbrennungsprozesse in jüngster Zeit immer mehr Brennverfahren mit Benzindirekteinspritzung durch. Gegenüber der konventionellen Saugrohreinspritzung erreichen Motoren mit dieser Technik derzeit eine Verbrauchseinsparung von bis zu 15 Prozent. Durch ihren Einsatz können heute bereits die mit der Einführung der Euro 4-Norm im Jahre 2005 in Europa geltenden verschärften Emissionsgrenzwerte sowie die Reduzierung des durchschnittlichen Kraftstoffverbrauchs auf 6 Liter pro 100km erreicht werden. Zusätzlich hat sich der Verband der europäischen Automobilhersteller (ACEA) 1998 verpflichtet, die 
mittlere $\mathrm{CO}_{2}$-Emission aller neuzugelassenen Pkw von $187 \mathrm{~g} / \mathrm{km}$ im Jahr 1995 um 25\% auf $140 \mathrm{~g} / \mathrm{km}$ im Jahr 2008 zu senken. Dies alles setzt ein detailliertes Verständnis des Gemischbildungsprozesses und des Verbrennungsvorgangs in Motoren mit Direkteinspritzung voraus, um eine weitere Optimierung dieses Brennverfahrens erreichen zu können. Eine der wichtigsten Größen bei der Entwicklung neuer Brennverfahren ist das lokale Mischungsverhältnis von Luft zu Kraftstoff, der sog. Lambda-Wert. Die Optimierung der zeitlichen Entwicklung und der dreidimensionalen Verteilung dieses Verhältnisses im Brennraum stellt heute eine wesentliche Aufgabe bei der Entwicklung neuer umwelt- und ressourcenschonender Brennverfahren dar.

Die Entwicklung laserspektroskopischer Messtechniken zur Analyse innermotorischer Vorgänge hat in den letzten Jahren einen entscheidenden Beitrag zum Verständnis der Gemischbildungsprozesse in Ottomotoren geliefert. Für den Einsatz dieser Techniken waren oftmals Modifikationen am Motor notwendig, um optische Zugänge in den Brennraum zu ermöglichen. Die sehr komplexen Gemischbildungsprozesse bei benzindirekteinspritzenden Motoren reagieren aber sehr empfindlich auf Veränderungen der äußeren Bedingungen. So hat beispielsweise jede Modifikation an der Geometrie oder den thermodynamischen Eigenschaften des Brennraums Auswirkungen auf den Gemischbildungsprozess.

Das Ziel dieser Arbeit ist es daher, ein optisches und damit berührungsloses Messsystem zur Bestimmung des Lambda-Wertes am Ort der Zündkerze zu entwickeln, welches keine Modifikationen des Motors für den Einsatz benötigt. Als einzigen Zugang zum Brennraum wird dabei die Zündkerzenbohrung verwendet. Die Möglichkeiten und Grenzen von Raman- und absorptionsspektroskopischen Techniken, welche eine hoch zeitaufgelöste Lambda-Wert-Bestimmung auch in Serienmotoren ermöglichen, sollen untersucht werden. 


\section{2. Überblick}

Im Rahmen dieser Arbeit werden zwei unterschiedliche Ansätze zur Analyse innermotorischer Gemischbildungsprozesse in benzindirekteinspritzenden Ottomotoren verfolgt. Zum einen wird dabei eine Raman-spektroskopische und zum anderen eine auf der Absorption von elektromagnetischer Strahlung im infraroten Wellenlängenbereich basierende Messtechnik entwickelt. Für beide Techniken werden Detektionssysteme beschrieben, die mit Hilfe von faseroptischen Sensoren Aufschluss über die Entwicklung des Lambda-Wertes am Ort der Zündkerze geben. Eine wesentliche Randbedingung für alle hier durchgeführten Arbeiten ist die Einsatzfähigkeit der entwickelten Messverfahren unter realen motorischen Bedingungen, insbesondere bei der Verwendung von Realkraftstoffen (z.B. Euro Super), tröpfchenbeladenen Strömungen und möglichst geringen Modifikationen der Motorgeometrie.

Nach einer kurzen Einführung in die physikalischen und technischen Grundlagen der Motorentechnik und verschiedener spektroskopischer und laserdiagnostischer Messmethoden an Ottomotoren wird in Kapitel 4 zunächst die Entwicklung einer Raman-Messtechnik vorgestellt, die es ermöglicht den Lambda-Wert des Luft/Kraftstoffgemisches örtlich und zeitlich aufgelöst zu bestimmen. Um die bei Anregung mit ultraviolettem Laserlicht durch die im Kraftstoff enthaltenen Aromaten auftretenden Fluoreszenzen zu vermeiden, wird auf eine Laseranregung mittels eines frequenzverdoppelten Nd:YAG-Lasers im sichtbaren Spektralbereich bei 532nm ausgewichen. Da der Raman-Streuquerschnitt und damit verbundene Emissionen sehr gering sind, werden neue Techniken entwickelt, die Laserintensität im Messvolumen zu erhöhen und die Effizienz der Detektion zu steigern. Der Einsatz der Raman-Spektroskopie war bislang nur in tröpfchenfreien Strömungen im Messvolumen möglich, da der Lambda-Wert nur für gasförmige Gemische definiert ist, die flüssigen Anteile aber immer Raman-Streulicht erzeugen. Grundlegende Untersuchungen dieser Emissionen aus Kraftstofftröpfchen werden durchgeführt und Möglichkeiten zur Unterdrückung der spontanen und stimulierten Raman-Streuung aufgezeigt, um eine fehlerfreie Bestimmung des Lambda-Wertes unter Realkraftstoffbedingungen in einem Ottomotor zu gewährleisten. Anschließend wird die Einsatzfähigkeit dieser Messtechnik an einem optisch zugänglichen Versuchsmotor unter Beweis gestellt. Dabei wurde das 1dimensional ortsaufgelöste Raman-Messsystem simultan mit einer flächigen Messung von UV-angeregter, laserinduzierter Fluoreszenz des Kraftstoffs kombiniert.

Um künftig in einem Serienmotor den Lambda-Wert bestimmen zu können, wird die vorgestellte Raman-Messtechnik zu einem faseroptischen Sensor weiterentwickelt, bei dem lediglich die Zündkerzenbohrung als optischer Zugang benötigt wird. Dafür werden grundlegende Untersuchungen über die auftretenden Probleme von Glasfasern unter Hochleistungs-Laseranregung durchgeführt. Erfolgreich zeigen dann Messungen an dem Versuchsmotor, dass mit diesem Detektionssystem auch während des Gemischbildungsprozesses im Motor eine Lambda-Wert-Bestimmung am Ort der Zündkerze erfolgen kann. 
Als zweiter Ansatz zur Lambda-Wert-Bestimmung werden in Kapitel 5 absorptionsspektroskopische Techniken vorgestellt. Dabei genügt eine einfache Halogenlampe um über die Absorption von infrarotem Licht bei 3,4 $\mu$ m die Konzentration von Kraftstoff bestimmen zu können. Auf diesem Messprinzip beruhend wird dann ein faseroptischer Sensor entwickelt, welcher anstelle einer Zündkerze in den Motor eingebaut werden kann. Erstmalig kann mit diesem Sensorsystem kurbelwinkelaufgelöst in einzelnen Motorzyklen das Luft/Kraftstoffverhältnis in einem Serienmotor am Ort der Zündkerze näherungsweise bestimmt werden. Gezeigt wird dabei die Einsatzfähigkeit der Messtechnik unter Realkraftstoffbedingungen und bei Motordrehzahlen von 2000U/min. Andere Molekülspezies, wie z.B. Wasser, haben dabei keinen Einfluss auf die Bestimmung des Lambda-Wertes. 


\section{2}

Einführung

\subsection{Ottomotorische Brennverfahren}

Die Verbrennung von Kraftstoff in Motoren ist ein exothermer Oxidationsprozess. Dabei verbinden sich die Kohlenwasserstoffteile Kohlenstoff und Wasserstoff mit Sauerstoff. Das Verhältnis der dem Motor tatsächlich zugeführten Luftmenge $m_{L}$ je Kraftstoffmenge $m_{K}$ zu der zur vollständigen Verbrennung dieser Kraftstoffmenge theoretisch benötigten Luftmenge $m_{L, \text { stö }}$ wird als Luftzahl oder Lambda-Wert $\lambda$ bezeichnet [38] und ist definiert als

$$
\lambda=\frac{\left(m_{L} / m_{K}\right)}{\left(m_{L} / m_{K}\right)_{s t o ̈}}=\frac{\left(m_{L} / m_{K}\right)}{\left(m_{L, s t o ̈} / m_{K}\right)}=\frac{m_{L}}{m_{L, s t o ̈}}
$$

mit:

$$
\begin{aligned}
m_{L} & : \text { vorliegende Luftmenge } \\
m_{K} & : \text { Kraftstoffmenge } \\
m_{L, \text { stö }} & : \text { zur vollständigen Verbrennung theoretisch benötigte Luftmenge }
\end{aligned}
$$

Eine vollständige und damit stöchiometrische Verbrennung erfolgt bei einem LambdaWert von 1. Bei einem Luftüberschuss nach der Verbrennung ist $\lambda>1$ („magerer" Motorbetrieb). Kommt es zu einem Luftmangel, d.h. es bleibt unverbrannter Kraftstoff übrig, ist $\lambda<1$ und man spricht von einem ,fetten" Gemisch. Bei einer stöchiometrischen Ver- 
brennung von Oktan beispielsweise ergibt sich folgende Reaktion:

$$
\mathrm{C}_{8} \mathrm{H}_{18}+12 \frac{1}{2} \mathrm{O}_{2} \rightarrow 8 \mathrm{CO}_{2}+9 \mathrm{H}_{2} \mathrm{O}
$$

Luft/Benzingemische können nur mit einem Lambda-Wert zwischen 0,6 und 1,6 gezündet werden [38]. Dagegen liegen die Zündgrenzen z.B. von Methanol bei 0,4 und 2,4.

In Ottomotoren älterer Bauart wird der Kraftstoff während der Ansaugphase vor dem Einlassventil des Zylinders in das Saugrohr eingespritzt. Zur Minimierung der LambdaWert-Zyklusschwankungen erfolgt dies über eine Mehrpunkt-Kraftstoffeinspritzung mit etwa 4bar Einspritzdruck [2]. Auf diese Weise gelangt bereits ein Gemisch von Luft und Kraftstoff in den Brennraum des Motors. Dem flüssigen Kraftstoff bleibt so genügend Zeit in der Einlass- und anschließenden Kompressionsphase zu verdampfen, so dass zum Zeitpunkt der Zündung ein homogenes Luft/Kraftstoffgemisch vorliegt. Um auch bei geringer Last eine optimale Verbrennung mit $\lambda=1 \mathrm{zu}$ erreichen, muss die angesaugte Luftmenge der geringeren eingespritzten Menge an Kraftstoff angepasst werden. Dazu wird mit Hilfe der Drosselklappe im Ansaugrohr der Luftdruck bei Teillastbetrieb abgesenkt. Gegen den dadurch im Brennraum entstehenden Unterdruck muss der in der Ansaugphase nach unten laufende Kolben somit Arbeit verrichten. Dies führt zu einer Verschlechterung des Wirkungsgrads eines Ottomotors durch Ladungswechselverluste [45]. Die Verbrennung des homogenen Luft/Kraftstoffgemisches erfolgt in diesen Motoren im gesamten Brennraum. Die über die Brennraumwände abgeführte Wärme verursacht einen weiteren, nicht unerheblichen Verlust.

Aus diesen Gründen ist in den letzten Jahren ein neues Brennverfahren entwickelt worden, welches über eine Benzin-Direkteinspritzung (BDE) in den Brennraum deutliche Kraftstoffeinsparungen ermöglicht und in Ottomotoren bereits serienmäßig eingesetzt wird. Bei dieser Motorentechnik kann auf eine Drosselung der Luftmenge weitestgehend verzichtet werden. Dadurch ist eine schnellere Regelung des Motors bei sich ändernder Last oder Drehzahl möglich. Da die Luftfüllung des Zylinders immer annähernd konstant ist, herrscht im Teillastbetrieb ein deutlicher Luftüberschuss mit $\lambda=2-4$. Durch eine direkte Einspritzung des Kraftstoffs in den Brennraum erst spät während der Kompressionsphase wird eine inhomogene Kraftstoffverteilung im Brennraum generiert. Man spricht dabei von einer Ladungsschichtung oder einem geschichteten Betrieb. Auch bei diesem Verfahren muss gewährleistet sein, dass trotz des Luftüberschusses außerhalb der Kraftstoffwolke ein zündfähiges Gemisch an der Zündkerze vorliegt. Da der Kraftstoff bei einer so späten Einspritzung wenig Zeit zum Verdampfen hat, wird dieser durch spezielle Hochdruck-Ventile mit einem Druck von 15-200bar eingespritzt, um so möglichst kleine Tröpfchen zu erzeugen. Für eine optimale Verbrennung mit $\lambda=1$ ist eine sehr genaue Anpassung des Einspritzdruckes, des Zeitpunktes der Einspritzung und der Menge des eingespritzten Kraftstoffs an die Motordrehzahl und die angelegte Last notwendig. Aus diesem Grund erfolgt die Steuerung eines BDE-Motors elektronisch.

Durch einen ungedrosselten Betrieb bis zu mittleren Drehzahlen werden im Teillastbereich bei diesem Brennverfahren Ladungswechselverluste vermieden. Zudem besitzt die 
lokal um die Zündkerze positionierte Kraftstoffwolke bei der Verbrennung kaum Wandkontakt, so dass die Wandwärmeverluste ebenfalls sehr gering sind. Dies beides führt zu einer erheblichen Steigerung des Wirkungsgrads bei Teillast und so zu einer Kraftstoffeinsparung von bis zu $15 \%$.

Allerdings kann der BDE-Motor bei höheren Drehzahlen und größerer Last durch hochturbulente Zylinderinnenströmungen und die Zündkerze benetzende, nicht verdampfte Kraftstofftröpfchen im geschichteten Betrieb nicht zündaussetzerfrei betrieben werden. Der Motor wird dann im sog. Homogenbetrieb gefahren. Dabei wird durch die Motorelektronik eine frühe Einspritzung während des Ansaugtaktes veranlasst und die Luftzufuhr über die Drosselklappe geregelt. Der Kraftstoff hat bei hohen Drehzahlen nur so genügend Zeit zu verdampfen. Unter Volllast erfolgt keine Drosselung der Luftmenge.

Bei BDE-Motoren gibt es verschiedene Verfahren, das Luft/Kraftstoffgemisch an der Zündkerze zu generieren und dem Kraftstoff über einen längeren Weg Zeit zum Verdampfen zu ermöglichen.

- Beim wandgeführten Brennverfahren wird der Kraftstoff aus dem seitlich positionierten Einspritzventil nach unten in Richtung der muldenförmigen Kolbenoberfläche gespritzt und von dieser wieder nach oben zur Zündkerze umgelenkt.

- Durch Klappen im Ansaugrohr und Mehrventiltechniken werden beim luftgeführten Brennverfahren Wirbel der einströmenden Luft im Brennraum erzeugt, welche den Kraftstoff leiten.

- Bei den strahlgeführten Brennverfahren wird der Kraftstoff in unmittelbarer Nähe zur Zündkerze eingespritzt und das Kraftstoffspray gegen Einspritzende am hinteren Teil des Sprays entzündet.

Alle diese direkteinspritzenden Brennverfahren sind sehr komplexe Systeme, die genaue Kenntnisse der Gemischbildungsprozesse erfordern, um einen optimalen Betrieb eines Ottomotors zu gewährleisten. Neben der Wahl einer Einspritztechnik (Drallventil, Mehrlochventil, usw.) ist beispielsweise auch die Lage und Ausrichtung der Einspritzventile (Injektoren) wichtig [57]. Die eingespritzte Kraftstoffmenge hängt von der Zeit zwischen dem Öffnen des Injektors am Ansteuerbeginn (ASB) und dem Schließen am Ansteuerende (ASE) sowie dem Einspritzdruck ab. In der Motorentechnik erfolgen alle Zeitangaben innerhalb eines Motorzyklus immer bezogen auf den Punkt der höchsten Kompression, dem sog. oberen Totpunkt (OT). Als Zeitpunkt gibt dabei der Winkel des Kurbelzapfens der Kurbelwelle zum OT in Grad Kurbelwinkel $\left({ }^{\circ} \mathrm{KW}\right)$ die Stellung des Kolbens wieder. Beispiel: $15^{\circ} \mathrm{KWvOT}=15$ Grad Kurbelwinkel vor oberem Totpunkt.

Eine nicht optimale Verbrennung führt zu einem erhöhten Auftreten von Schadstoffen. In fetten Gemischen erfolgt die Verbrennung des Kraftstoffs nur unvollständig und unverbrannte Kraftstoff-Moleküle führen zur Bildung von Rußpartikeln und Kohlenmonoxid im Abgas. Bei mageren Luft/Kraftstoffgemischen verbindet sich der unverbrannte, 
überschüssige Restsauerstoff im heißen Brennraum mit an der Verbrennung unbeteiligtem Stickstoff zu besonders schädlichen und unerwünschten Stickoxiden [5]:

$$
\mathrm{N}_{2}+\mathrm{O}_{2} \rightarrow 2 \mathrm{NO} \quad \text { oder } \quad \frac{1}{2} \mathrm{~N}_{2}+\mathrm{O}_{2} \rightarrow 2 \mathrm{NO}_{2}
$$

Für die Wahl des optimalen Zündzeitpunktes ist es notwendig, den Lambda-Wert und dessen zeitliche Entwicklung am Ort der Zündkerze zu kennen. In Kapitel 4 wird eine Methode vorgestellt, mit der dieser Wert an einem Transparentmotor mittels spontaner Raman-Streuung bestimmt werden kann. Eine Modifikation des Motors durch das Einsetzen von zusätzlichen optischen Zugängen, wie Glasfenstern, beeinflusst die Temperaturverteilung im Brennraum durch unterschiedliche Wärmeleitfähigkeitskoeffizienten der Materialien und darüber das Verdampfungsverhalten und den Gemischbildungsprozess des Kraftstoffs. Die innermotorischen Bedingungen in einem solchen Motor entsprechen somit nur teilweise denen in einem Serienmotor. Daher ist die Entwicklung eines miniaturisierten Messsystems, welches nur die Zündkerzenbohrung als einzigen Zugang zum Brennraum verwendet, das Ziel dieser Arbeit. 


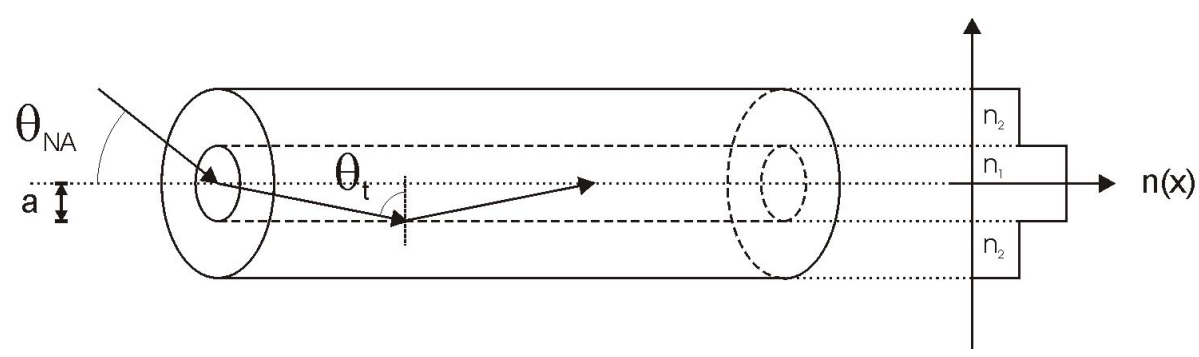

Abbildung 2.1.: Schematischer Aufbau einer Glasfaser mit Stufenindexprofil

\subsection{Lichtwellenleiter}

Das Prinzip der Ausbreitung von Lichtwellen in einem sog. Lichtwellenleiter beruht auf der Brechung des Lichtes beim Übergang zwischen zwei Materialien mit verschiedenen Brechungsindizes. Ein Wellenleiter mit Stufenindexprofil, z.B. eine Glasfaser, besteht aus einem Kern (engl. core) mit höherer Brechzahl $n_{1}$ und Durchmesser $2 a$, der von einem Mantel (engl. cladding) mit niedrigerer Brechzahl $n_{2}$ umgeben ist. Der Aufbau einer solchen Glasfaser ist in Abbildung 2.1 schematisch dargestellt. Bei dieser wird das Licht durch Vielfachreflexion an der Grenzschicht zwischen dem optisch dichteren Kern und dem optisch dünneren Mantel geleitet. Der Grenzwinkel für die Totalreflexion $\left(\theta_{2}=90^{\circ}\right)$ für Licht im Kern am Mantel ist dabei gegeben durch das Snelliussche Brechungsgesetz:

$$
\sin \theta_{t}=\frac{n_{2}}{n_{1}}
$$

Quarzglasfasern werden aus hochreinem $\mathrm{SiO}_{2}$ hergestellt. Dabei kann der Brechungsindex des Glases durch eine geeignete Dotierung mit Oxiden während des Herstellungsprozesses verändert werden. Beispielsweise führt der Einbau von Fluor $(\mathrm{F})$ oder Bortrioxid $\left(\mathrm{B}_{2} \mathrm{O}_{3}\right)$ zu einer niedrigeren Brechzahl. Dagegen kann mit Germaniumoxid $\left(\mathrm{GeO}_{2}\right)$ oder Phosphorpentoxid $\left(\mathrm{P}_{2} \mathrm{O}_{5}\right)$ eine höhere Brechzahl erzielt werden.

Der Kerndurchmesser bei Standard-Telekommunikationsfasern beträgt 8,3 $\mu \mathrm{m}$ und der Durchmesser des Mantels 125 $\mu \mathrm{m}$. Der Brechzahlunterschied (bei Standardfasern 0,36\%) bewirkt, dass genau das Licht in der Glasfaser geführt wird, welches innerhalb eines maximalen Akzeptanzwinkels $\theta_{N A}$ in die Faser eingestrahlt wird. Dieser Grenzwinkel für die Einkopplung am Faserende legt die numerische Apertur NA der Faser fest:

$$
\mathrm{NA}=n_{0} \sin \theta_{N A}=\sqrt{n_{1}^{2}-n_{2}^{2}}
$$

wobei $n_{0}$ die Brechzahl der Umgebung, z.B. Luft, ist. Die numerische Apertur gibt in Einmodenfasern sowie in voll ausgeleuchteten Mehrmodenfasern gleichzeitig an, mit welchem Divergenzwinkel das geführte Licht am Faserende wieder austritt. Bei den meisten Quarzglasfasern liegt die NA bei 0,22. 
Die Beschreibung der Lichtwellenleitung nach den Regeln der geometrischen Optik setzt voraus, dass die Abmessungen der Faser groß gegenüber der Lichtwellenlänge sind. Da diese Bedingung beispielsweise bei den in der Nachrichtentechnik verwendeten Wellenlängen zwischen $1,2 \mu \mathrm{m}$ und 1,6 $\mathrm{m}$ nicht erfüllt ist, muss der Wellencharakter des Lichtes berücksichtigt werden. Dabei zeigt sich, dass nur solche Wellenfronten im Lichtleiter geführt werden, die sich nach der Reflexion an der Grenzfläche konstruktiv überlagern. Die diese Bedingung erfüllende elektromagnetische Welle wird als Mode bezeichnet. Welche Moden im Faserkern geführt werden, hängt vom Kernradius $a$, der Wellenlänge $\lambda$ und der numerischen Apertur der Faser ab. Für die normierte Frequenz $V$ als charakteristische Größe gilt dabei [54]:

$$
V=\frac{2 \pi}{\lambda} a \sqrt{n_{1}^{2}-n_{2}^{2}}=\frac{2 \pi}{\lambda} a \mathrm{NA} .
$$

Von $V=0$ an führt die Faser nur eine Mode, die sog. Grundmode. Erst wenn $V$ einen Grenzwert $V_{C}=2,405$ überschreitet, können sich höhere Moden ausbreiten. Der Grenzwert entspricht der ersten Nullstelle der die Feld- und Intensitätsverteilung beschreibenden Besselfunktion. Für Wellenlängen oberhalb der zu $V_{C}$ gehörenden Cut-Off-Wellenlänge $\lambda_{C}$ ist die Faser eine Einmodenfaser (engl. single-mode-fiber). Mehrmodenfasern (engl. multi-mode-fiber) können Kerndurchmessern von bis zu 1,5mm haben.

Eine charakteristische Größe für Lichtwellenleiter ist die Dämpfung, also der Verlust von Energie bei der Übertragung elektromagnetischer Wellen. So reduziert sich die Leistung $P_{0}$ nach einer Faserlänge L auf

$$
P_{T}=P_{0} \exp (-\alpha L)
$$

mit der Dämpfungskonstante $\alpha$. Typischerweise wird der Dämpfungskoeffizient in der logarithmischen Einheit Dezibel [dB] angegeben:

$$
\alpha_{d B}=-10 \log \left(\frac{P_{T}}{P_{0}}\right)
$$

Dies hat den Vorteil, dass sich verschiedene Dämpfungswerte einfach addieren lassen. Üblicherweise wird daher die Dämpfung einer Faser in $\mathrm{dB} / \mathrm{km}$ angegeben.

Für die Dämpfung optischer Signale in einer Glasfaser gibt es verschiedene Ursachen. So treten infolge der Wechselwirkung zwischen der Lichtwelle und den Elektronen und Atomen des Materials Leistungsverluste durch Absorptionsdämpfung auf. Bei Lichtwellenleitern aus Quarzglas nimmt die Absorption durch starke Absorptionsbanden des Materials und der Dotierstoffe unterhalb von $1 \mu \mathrm{m}$ und oberhalb von $1,6 \mu \mathrm{m}$ Werte größer als $0,1 \mathrm{~dB} / \mathrm{km}$ an [29]. Neben der Absorptionsdämpfung treten weitere Verluste durch Rayleigh-Streuung an Dichteschwankungen des Glases und eingelagerten Dotierstoffen auf. 


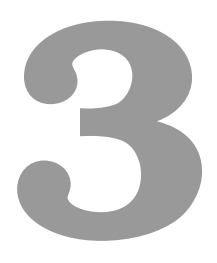

\section{Theoretische Grundlagen der angewandten Messverfahren}

In diesem Kapitel werden die verschiedenen spektroskopischen Messtechniken vorgestellt, die im Rahmen der Entwicklung eines optischen Sensors zur Bestimmung des Luft/Kraftstoffverhältnisses in Ottomotoren eingesetzt wurden. Neben den physikalischen Grundlagen dieser Verfahren sollen deren Möglichkeiten und Grenzen zur Lambda-WertBestimmung erläutert werden.

\subsection{Raman-Spektroskopie}

Die Verbrennungsdiagnostik mittels Raman-spektroskopischer Messmethoden besitzt gegenüber anderen Spektroskopietechniken eine Reihe von Vorteilen. Da es sich um ein nicht resonantes Messverfahren handelt, ist beipielsweise zur gleichzeitigen Detektion aller Molekülspezies eines Gemisches nur eine Anregungswellenlänge notwendig. Dabei kann jede Wellenlänge verwendet werden, ohne eine Abstimmung auf Resonanzen einer Molekülart. Bei der Raman-Streuung handelt es sich um einen nahezu instantanen inelastischen Streuprozess, so dass die Raman-Streulichtintensitäten einer Gemischkomponente unabhängig von den übrigen Spezies und Stößen mit anderen Molekülen des Gemisches im Messvolumen sind. Allerdings ist dieses Streulicht im Vergleich zu resonanten Prozessen extrem schwach. 


\subsubsection{Theoretische Grundlagen}

Licht, welches ein Gas, eine Flüssigkeit oder einen transparenten Festkörper durchstrahlt, tritt immer in Wechselwirkung mit den Atomen, Molekülen oder Gitterstrukturen des Mediums. Die einfallende Welle induziert dabei ein elektrisches Dipolmoment $\overrightarrow{p_{i n d}}$, das proportional zur elektrischen Feldstärke $\vec{E}$ ist und ein eventuell bereits im Molekül vorhandenes permanentes Dipolmoment $\overrightarrow{p_{0}}$ überlagert. In das gesamte Dipolmoment

$$
\vec{p}=\overrightarrow{p_{0}}+\alpha \cdot \vec{E}
$$

geht dabei die Polarisierbarkeit $\alpha$ des Moleküls ein. Dieser Tensor setzt sich zusammen aus einem festen Anteil $\alpha_{0}$ und einem Anteil, der von der relativen Lage $Q_{n}$ der Kerne des Moleküls zueinander abhängt. Das Dipolmoment lässt sich damit nach den Normalkoordinaten entwickeln. Es gilt:

$$
\begin{gathered}
\alpha=\alpha_{0}+\sum_{n}\left(\frac{\partial \alpha}{\partial Q_{n}}\right)_{0} Q_{n} \\
\vec{p}=\vec{p}_{0}+\sum_{n}\left(\frac{\partial \vec{p}}{\partial Q_{n}}\right)_{0} Q_{n}
\end{gathered}
$$

Die Normalkoordinaten können für kleine Schwingungsamplituden durch harmonische Schwingungen mit Amplituden $Q_{n, 0}$ und den Frequenzen $\omega_{n}$ beschrieben werden:

$$
Q_{n}(t)=Q_{n, 0} \cdot \cos \omega_{n} t
$$

Das äußere elektrische Feld des einfallenden Lichtes mit der Frequenz $\omega$

$$
\vec{E}(t)=\overrightarrow{E_{0}} \cdot \cos \omega t
$$

führt somit zu einem zeitabhängigen elektrischen Dipolmoment im Molekül [13]:

$$
\begin{aligned}
\vec{p}=\overrightarrow{p_{0}} & +\sum_{n}\left(\frac{\partial \vec{p}}{\partial Q_{n}}\right)_{0} Q_{n, 0} \cdot \cos \omega_{n} t \\
& +\alpha_{0} \overrightarrow{E_{0}} \cdot \cos \omega t \\
& +\left(\sum_{n}\left(\frac{\partial \alpha}{\partial Q_{n}}\right)_{0} Q_{n, 0} \cdot \cos \left(\omega \pm \omega_{n}\right) t\right) \cdot \frac{\vec{E}_{0}}{2}
\end{aligned}
$$

Die ersten beiden Terme beschreiben das permanente Dipolmoment und den mit den Molekülschwingungen oszillierenden Anteil. Letzterer ist verantwortlich für die Emission und Wechselwirkung des Moleküls mit Licht im infraroten Wellenlängenbereich (siehe Abschnitt 3.3). Der nächste Term beschreibt die Rayleigh-Streuung. Die einfallende Welle 
führt zu einem induzierten Dipolmoment im Molekül, welches daraufhin als oszillierender elektrischer Dipol elektromagnetische Wellen auf der induzierten Oszillationsfrequenz $\omega$ abstrahlt. Dabei hängt die Amplitude der elastischen Streuwelle von der Polarisierbarkeit des Moleküls in Richtung $\vec{E}_{0}$ des äußeren elektrischen Feldes ab.

Der letzte Term beschreibt den Einfluss des induzierten Dipolmoments auf die Kernschwingungsfrequenz $\omega_{n}$. Die dadurch ebenfalls entstehende Dipolstrahlung der inelastischen Raman-Streuung wird durch die Abhängigkeit der Polarisierbarkeit von den Auslenkungen der Kerne bestimmt. Das Molekül wird als Raman aktiv bezeichnet, wenn

$$
\left(\frac{\partial \alpha}{\partial Q_{n}}\right)_{0} \neq 0
$$

gilt. In diesem Fall treten zusätzlich neben der Rayleigh-Streuung jeweils rot - und blauverschobene Raman-Spektrallinien auf. Aus historischen Gründen werden die Spektrallinien mit den Frequenzen $\left(\omega-\omega_{n}\right)$ als Stokes-Linien und die mit $\left(\omega+\omega_{n}\right)$ als Anti-Stokes-Linien bezeichnet. Bei den letzteren erfolgt ein Energietransfer vom Molekül an das einfallende Photon. Daher können Anti-Stokes-Linien nur bei höheren Temperaturen auftreten, wenn höhere Energieniveaus des Moleküls thermisch besetzt sind.

Das Molekül wirkt durch das sich periodisch mit der Zeit ändernde induzierte Dipolmoment wie ein Herzscher Dipol, dessen abgegebene Strahlungsintensität pro Raumwinkel gegeben ist durch:

$$
I^{\Omega}(\theta)=\frac{|\vec{p}|^{2} \omega^{4} \sin ^{2} \theta}{32 \pi^{2} \epsilon_{0} c^{3}}
$$

Dabei ist $\theta$ der relative Abstrahlwinkel bezüglich des durch den Polarisierbarkeitstensor $\alpha$ des Moleküls vorgegebenen induzierten Dipolmoments $\vec{p}$. Die Strahlungsintensität des Streulichts mit gleicher Polarisationsrichtung wie das Anregungslicht ist um so größer, je geringer die Richtungsabweichung des Dipolmoments zum äußeren Feld $\vec{E}$ ausfällt.

Quantenmechanisch betrachtet, verursacht ein einfallendes Photon bei der RamanStreuung in der stabilen Wellenfunktion der Elektronenhülle eines Moleküls eine Störung. Ist die Photonenenergie zu gering für eine elektronische und zu hoch für eine vibratorische oder rotatorische Anregung, kann kein Übergang in ein stabiles Energieniveau sondern nur in einen extrem kurzlebigen Zwischenzustand erfolgen. In der klassischen Betrachtung repräsentieren diese eigentlich nicht erlaubten Zwischenzustände das induzierte Dipolmoment. Die Elektronenhülle relaxiert in einen erlaubten Zustand in weniger als $10^{-12} \mathrm{~s}$ und sendet dabei ein Photon aus. Der Streuprozess erfolgt somit nahezu instantan. Eine strahlungslose Entvölkerung der Zwischenzustände durch Molekülstöße ist durch deren extrem kurze Lebensdauer daher auch bei hohen Drücken nahezu ausgeschlossen. Allerdings ist der Anteil der inelastischen Raman-Streuung um etwa einen Faktor 1000 geringer als der der Rayleigh-Streuung.

Die Energie eines Moleküls kann sich nur auf quantenmechanisch definierte Zustände verteilen. Ein Energieaustausch zwischen dem Photon und dem Molekül ist also nur 
in der Größe erlaubter Vibrations- oder Rotationsübergänge möglich, für die folgende Auswahlregeln gelten:

$$
\begin{aligned}
& \Delta v=0 \quad \Delta J= \pm 2 \quad \text { für die Rotations-Raman-Streuung und } \\
& \Delta v= \pm 1 \quad \Delta J=0, \pm 2 \quad \text { für die Vibrations-Rotations-Raman-Streuung }
\end{aligned}
$$

Bei einer monochromatischen Anregung führen diese Auswahlregeln somit zu einem diskreten Emissions-Linienspektrum. Die Frequenzverschiebung der Raman-Linien ist gegenüber der Rayleigh-Linie bei einem Molekül unabhängig von der Frequenz des eingestrahlten Lichts, da die individuellen intramolekularen Kräfte einer Molekülspezies die Energieabstände der verschiedenen Niveaus bestimmen. Das Spektrum einer Raman-Streuung an einem Molekül ist also charakteristisch für diese Spezies und liefert eine Art spektralen „Fingerabdruck“. So lassen sich einzelne Komponenten innerhalb eines Stoffgemischs anhand ihrer charakteristischen Linien im Raman-Spektrum identifizieren.

Sowohl die Rayleigh- als auch die Raman-Streuung sind lineare Streuprozesse, d.h. die Streulichtintensität hängt linear von der Anzahl der am Streuprozess beteiligten Moleküle ab. Beide bieten daher die Möglichkeit in einem Gemisch verschiedener Spezies Moleküldichten zu bestimmen. Dabei ist das Rayleigh-Signal ein Maß für die Gesamtdichte innerhalb eines Messvolumens, wo hingegen die Raman-Streuung die Konzentration einzelner Komponenten wiedergibt. Die Streulichtintensität einer Raman-Spektrallinie ist gegeben als

$$
I_{\text {Raman }, i}=\eta_{c} I_{0} \Omega V_{c} N_{i}\left(\frac{\partial \sigma}{\partial \Omega}\right)_{\text {Raman }, i}
$$

mit

$$
\begin{aligned}
\eta_{c} & : \text { Quanteneffizienz des Detektionssystems } \\
I_{0} & : \text { eingestrahlte Laserintensität }\left[\mathrm{W} / \mathrm{cm}^{2}\right] \\
N_{i} & : \text { Anzahl an Molekülen der Spezies } i\left[\mathrm{~cm}^{-3}\right] \\
\Omega & : \text { Raumwinkel der Detektionsoptik }[\mathrm{sr}] \\
V_{c} & : \text { Detektionsvolumen }\left[\mathrm{cm}^{3}\right] \\
\left(\frac{\partial \sigma}{\partial \Omega}\right)_{\text {Raman }, i}: & \text { Raman-Streuquerschnitt der Molekülspezies } i
\end{aligned}
$$

Die Besetzung der Vibrations- und Rotationsniveaus ist abhängig von der Temperatur und wird durch eine Boltzmannverteilung beschrieben. Dabei ergibt sich, dass obere Vibrationsniveaus erst bei höheren Temperaturen nennenswert besetzt werden. Beispielsweise ist bei Stickstoffmolekülen das 1. Vibrationsniveau erst ab einer Temperatur von etwa 600K zu 1\% besetzt [15]. Ein Temperatureinfluss auf die Streulichtintensität existiert daher erst deutlich oberhalb dieser Temperatur und in Gleichung 3.10 muss dementsprechend die Temperaturabhängigkeit des Raman-Streuquerschnitts mit berücksichtigt werden [64].

Die Intensität der induzierten Dipol-Strahlung eines Moleküls skaliert gemäß Gleichung 3.8 mit der vierten Potenz der Schwingungsfrequenz des induzierten Dipolmoments und 
damit mit der Frequenz des eingestrahlten Lichtes. Da in gleicher Weise auch der Rayleighund Raman-Streuquerschnitt mit der Frequenz des Lichtes ansteigt, werden Laser mit ultravioletter Strahlung vielfach als Lichtquelle in Streulichtexperimenten eingesetzt.

Liegen sehr hohe Leistungsdichten der Laseranregung in einem Messvolumen vor, so kann stimulierte Raman-Streuung (SRS) auftreten. Trotz der extrem kurzen Lebensdauer der verbotenen Zwischenzustände wird dabei ein angeregtes Molekül von dem spontan emittierten Raman-Photon eines anderen Moleküls zur Abgabe eines weiteren Photons gebracht. In Flüssigkeiten und Gasen erfolgt die stimulierte Raman-Streuung vornehmlich in Vorwärts- und Rückwärtsrichtung des eingestrahlten Laserlichtes, da sich beide angeregten Moleküle im Laserstrahl befinden. 


\subsubsection{Lambda-Wert-Bestimmung}

Die Energieabstände der erlaubten vibratorischen und rotatorischen Zustände hängen von den Kernmassen und -abständen in einem Molekül sowie von den innermolekularen Bindungsstärken ab und sind für Vibrationsniveaus deutlich größer als für Rotationsniveaus. Das Schwingungs-Rotations-Spektrum von Übergängen zwischen Schwingungs-RotationsNiveaus $\left(v_{i}, J_{i}\right) \leftrightarrow\left(v_{k}, J_{k}\right)$ innerhalb desselben elektronischen Zustandes mit $v_{i} \neq v_{k}$ liegt im infraroten Spektralbereich $(2-10 \mu m)$, das reine Rotationsspektrum für $v_{i}=v_{k}$ dagegen im Mikrowellenbereich. Durch diese größere Energieverschiebung lassen sich Vibrationsniveaus spektral deutlich einfacher trennen.

Im Rahmen dieser Arbeit waren insbesondere die Stokes-Ramanlinien 1.Ordnung von Bedeutung. Die Schwingungsfrequenzen und Raman-Streuquerschnitte bei zwei Anregungswellenlängen verschiedener für die motorische Verbrennung bedeutsamer Moleküle sind in Tabelle 3.1 zusammengestellt.

Der Lambda-Wert eines Gemischs ist, wie in Kapitel 2.1 gezeigt, über das Luft/Kraftstoffverhältnis definiert. Zwar ist der Streuquerschnitt von Sauerstoff etwas größer als der von Stickstoff, doch in Raumluft ist die Teilchenzahl pro Mol Luft von $\mathrm{N}_{2}$ fast 4 mal größer. Da die Raman-Streulichtintensität mit der Anzahl der Moleküle einer Spezie skaliert, ist es daher ratsam als Repräsentant des Luftanteils die intensivere Stickstofflinie zu wählen. Bei der Bestimmung des Lambda-Wertes eines Gemisches ist eine solche indirekte Messung immer dann zulässig, wenn sich keine Abgase im Messvolumen befinden, bzw. die Verbrennung noch nicht eingesetzt hat.

\begin{tabular}{|c|c|cc|}
\hline Molekül & $\begin{array}{c}\text { Schwingungs- } \\
\text { frequenz }\left[\mathrm{cm}^{-1}\right]\end{array}$ & $\begin{array}{c}\text { Streuquerschnitt } \\
{\left[\times 10^{-30} \mathrm{~cm}^{2} / \mathrm{sr}\right]}\end{array}$ \\
\hline & & $248 \mathrm{~nm}$ & $532 \mathrm{~nm}$ \\
\hline $\mathrm{N}_{2}$ & 2331 & 13,0 & 0,46 \\
$\mathrm{O}_{2}$ & 1556 & 16,6 & 0,65 \\
$\mathrm{CO}_{2}$ & 1388 & 15,1 & 0,60 \\
$\mathrm{CH}_{4}$ & 3017 & 53,7 & 1,72 \\
$\mathrm{H}_{2} \mathrm{O}$ & 3657 & 30,8 & 0,89 \\
\hline
\end{tabular}

Tabelle 3.1.: Charakteristische Schwingungsfrequenzen und Raman-Streuquerschnitte verschiedener Moleküle bei zwei unterschiedlichen Anregungswellenlängen (Quelle: [63]) 
Die Streulichtintensität der Raman-Kraftstofflinie ergibt sich nach Gleichung 3.10 für $N_{K}$ Kraftstoffmoleküle im Messvolumen zu:

$$
I_{K}=\eta_{c} \cdot \Omega \cdot V_{c} \cdot\left(\frac{\partial \sigma_{K, C H}}{\partial \Omega}\right) \cdot I_{0} \cdot N_{K}=c_{K} \cdot I_{0} \cdot N_{K}
$$

und analog für $N_{N_{2}}$ Stickstoffmoleküle:

$$
I_{N_{2}}=\eta_{c} \cdot \Omega \cdot V_{c} \cdot\left(\frac{\partial \sigma_{N_{2}}}{\partial \Omega}\right) \cdot I_{0} \cdot N_{N_{2}}=c_{N_{2}} \cdot I_{0} \cdot N_{N_{2}}
$$

Das Verhältnis der beiden Intensitäten hängt also von der Anzahl der Stickstoff- und Kraftstoffmoleküle im Messvolumen ab und ist bis auf die Konstanten $c_{K}$ und $c_{N_{2}}$

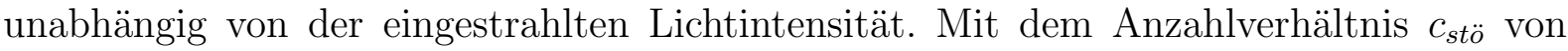
Kraftstoff- zu Sauerstoffmolekülen bei einer stöchiometrischen Verbrennung ergibt sich für den Lambda-Wert daraus:

$$
\lambda=c_{s t \ddot{o}} \cdot \frac{N_{N_{2}}}{N_{K}}=c_{s t \ddot{o}} \cdot \frac{c_{K} I_{N_{2}}}{c_{N_{2}} I_{K}}=c \cdot \frac{I_{N_{2}}}{I_{K}}
$$

Die Bestimmung des Lambda-Wertes ist also bis auf eine Konstante $c$ aus dem Verhältnis der Raman-Streulichtintensitäten von Stickstoff und Kraftstoff möglich.

Werden beide Raman-Linien simultan detektiert, wie es bei der Verwendung eines Mehrkanalanalysators (optical multichannel analyzer, OMA) der Fall ist, so wirken sich alle die Messung beeinflussenden Störfaktoren wie beispielsweise Schwankungen der Laserintensität, Verschmutzungen der Optik, Abbildungsfehler oder konstante Hintergrundsignale, auf beide Signalintensitäten in identischer Weise aus. Dies hat den großen Vorteil, dass alle diese Störgrößen bei der zur Bestimmung des $\lambda$-Wertes durchgeführten Verhältnisbildung der Lininenintensitäten herausfallen. Für den Druck gilt dies ebenso, da die Anzahldichten beider Molekülspezies identisch mit einer Druckänderung skalieren.

Diese simultane Detektion der Raman-Linien ermöglicht also eine hoch genaue quantitative Messung des Lambda-Wertes. Leider relativieren die sehr niedrigen RamanSignalintensitäten diesen Vorteil ein wenig. 


\subsection{Laserinduzierte Fluoreszenz}

Mit der im voran gegangenen Kapitel beschriebenen spontanen Raman-Streuung ist auf Grund der geringen Signalintensitäten eine Bestimmung sehr geringer Moleküldichten extrem schwierig. Auch mit Hilfe stimulierter spektroskopischer Messmethoden, wie der Coherent Anti-Stokes Raman Spectroscopy (CARS), können nur Konzentrationen > 0, 1\% (1000ppm) bestimmt werden. Typischerweise liegt die Konzentration von bei einer Verbrennung auftretenden Radikalen, wie z.B. OH, CH, NH oder CN, unter 0,01\% (100ppm) [15]. Laserinduzierte Fluoreszenz besitzt dagegen auf Grund der sehr viel höheren Streuquerschnitte die Möglichkeit auch bei so geringen Konzentrationen diese Molekülspezies zu detektieren. Auch eine flächige Visualisierung von Kraftstoffdichteverteilungen kann durch laserinduzierter Fluoreszenz (LIF) erfolgen. Durch die Zugabe von Exciplexen zum Kraftstoff ist es desweiteren möglich, mit LIF eine 2-dimensionale Temperaturbestimmung in Sprays sowie eine flächige Analyse der flüssigen und gasförmigen Kraftstoffverteilung im Brennraum eines Motors zu erzielen [35].

\subsubsection{Theoretische Grundlagen}

Der Übergang zwischen zwei elektronischen Niveaus in einem Molekül ist möglich, wenn die Energie eines absorbierten Photons der Energiedifferenz der beiden Zustände entspricht. Dabei liegt die Wellenlänge solcher Photonen im sichtbaren und ultravioletten Spektralbereich. Wird für die Anregung ein Laser als Lichtquelle verwendet, spricht man von laserinduzierter Fluoreszenz (LIF). Durch spontane Emission kann das angeregte Molekül nach einer gewissen Zeit unter Ausstrahlung eines Photons wieder in einen niedrigeren energetischen Zustand übergehen. Liegt dieses Energieniveau oberhalb des Ausgangsniveaus wird das Fluoreszenzlicht rot-verschoben. Bei höheren Temperaturen kann auf Grund der Besetzung auch ein Übergang in ein niedrigeres Niveau erfolgen. Das emittierte Photon ist dann blau-verschoben. Die als Fluoreszenzlebensdauer bezeichnete Zeit in der sich das Molekül im oberen Niveau befindet, liegt zwischen $10^{-10} \mathrm{~s}$ und $10^{-5} \mathrm{~s}$. Übergänge zwischen Vibrations- oder Rotationsniveaus innerhalb eines elektronischen Zustandes können strahlungslos erfolgen. Der Abstand der Energieniveaus ist dabei von den innermolekularen Kräften abhängig. So besitzt ein zweiatomiges Molekül, wie z.B. $O_{2}$, ein Absorptionsspektrum mit diskreten Linien. Die mit der großen Anzahl an Freiheitsgraden in größeren Molekülen verbundene hohe Dichte an erlaubten Übergängen dagegen führt zu kontinuierlichen Absorptionsspektren.

Die lange Fluoreszenzlebensdauer hat zur Folge, dass im Gegensatz zur RamanStreuung hier zwischen der Anregung des Moleküls und der Aussendung eines Photons Stöße mit anderen Molekülen auftreten können. Dabei erfolgt ein Energieübertrag auf ein oder mehrere andere Moleküle und das ursprünglich angeregte Molekül kann so strahlungslos wieder in den Grundzustand übergehen. Die Fluoreszenzlichtintensität eines Molekülgemischs wird dadurch verringert. Dieser Effekt wird als Fluoreszenzlöschung oder 
Quenching bezeichnet.

Wie bei der Raman-Streuung kann eine Abregung der elektronisch angeregten Zustände bei einer sehr hohen Energiedichte auch stimuliert erfolgen. Dieser Effekt wird z.B. bei Lasern ausgenutzt.

In erster Näherung können die Wechselwirkungsprozesse von elektromagnetischer Strahlung mit einem Atom, Ion oder Molekül und die damit verbundenen Absorptionsund Emissionsprozesse zwischen einem elektronischen Grundzustand und einem angeregten Zustand durch ein Zwei-Niveau-Modell beschrieben werden. Bei diesem vereinfachten Modell werden Vibrations- und Rotationsübergänge innerhalb eines elektronischen Zustands oder die Entleerung des Grundzustandes bei sehr hoher Anregungsintensität (Sättigung) nicht berücksichtigt.

Die Wahrscheinlichkeiten dafür, dass als Wechselwirkung von einem eingestrahlten Photon mit einem Molekül eine Absorption $\left(W_{12}\right)$ oder eine stimulierte Emission $\left(W_{21}\right)$ erfolgt, hängt vom zeitlichen Intensitätsverlauf $I(t)$ des Laserpulses und dem Überlappungsintegral $g\left(\nu_{a b s}, \nu_{\text {laser }}\right)$ des spektralen Profils des Lasers und der spektralen Breite der beteiligten Absorptionslinien/-banden ab. Mit den Einsteinkoeffizienten für die Absorption $B_{12}$ und die stimulierte Emission $B_{21}$ gilt:

$$
\begin{aligned}
& W_{12}(t)=B_{12} I(t) g\left(\nu_{a b s}, \nu_{\text {laser }}\right) \\
& W_{21}(t)=B_{21} I(t) g\left(\nu_{a b s}, \nu_{\text {laser }}\right) .
\end{aligned}
$$

Die spontane Emission ist unabhängig von einem äußeren Strahlungsfeld. Die Wahrscheinlichkeit dafür ist durch den Einsteinkoeffizienten $A_{21}$ gegeben. Die Gesamtbesetzungsdichte eines Molekülensembles ist $N=N_{1}(t)+N_{2}(t)$ mit Besetzungsanzahl $N_{1}$ des unteren Zustandes und $N_{2}$ der Anzahl der angeregten Moleküle, wobei $N_{2}(t=0)=0$ gilt. Mit der durch Stöße verursachten Quenchingrate $Q_{21}$ ergibt sich für die Ratengleichungen der Besetzungsdichte $N_{2}$ :

$$
\frac{d N_{2}(t)}{d t}=W_{12}(t) N_{1}(t)-N_{2}(t)\left[W_{21}(t)+A_{21}+Q_{21}\right]
$$

Geht man von einer Laseranregung mit einem Rechteckpuls der Länge $\tau$ aus, so erhält man nach Integration die zeitliche Entwicklung der Besetzung des angeregten Zustandes während der Laseranregung $(0<t<\tau)$ :

$$
N_{2}(t)=\frac{N W_{12}}{\left(A_{21}+Q_{21}\right)}\left[1-e^{-\left(A_{21}+Q_{21}\right) t}\right]
$$

unter der Annahme, dass $W_{12}$ und $W_{21} \ll\left(A_{21}+Q_{21}\right)$ und der Randbedingung $N_{2}(t=$ $0)=0$. Für die Fluoreszenz nach dem Abklingen des Laserpulses $(\tau<t<\infty)$ ergibt sich:

$$
N_{2}(t)=N_{2}(\tau) e^{-\left(A_{21}+Q_{21}\right)(t-\tau)}
$$


Die gesamte durch spontane Emission abgestrahlte Fluoreszenzintensität $S_{f}$ aus dem Volumen $V$ ist dann:

$$
\begin{aligned}
S_{f}= & \int_{0}^{\infty} N_{2}(t) A_{21} V d t \\
= & \int_{0}^{\tau} A_{21} \frac{N W_{12}}{\left(A_{21}+Q_{21}\right)}\left[1-e^{-\left(A_{21}+Q_{21}\right) t}\right] V d t \\
& +\int_{\tau}^{\infty} A_{21} N_{2}(\tau) e^{-\left(A_{21}+Q_{21}\right)(t-\tau)} V d t
\end{aligned}
$$

Dabei ist der Term $q_{f}:=A_{21} /\left(A_{21}+Q_{21}\right)$ die Quantenausbeute der Fluoreszenz und wird auch als Stern-Vollmer-Faktor bezeichnet. Unter der Annahme, dass die Laserintensität zeitlich einem Rechteckpuls mit $E=I \tau$ folgt, ergibt sich dann aus der Integration die gesamte Fluoreszenzintensität zu:

$$
S_{f}=B_{12} E g\left(\nu_{a b s}, \nu_{l a s e r}\right) N \frac{A_{21}}{A_{21}+Q_{21}}
$$

\subsubsection{Lambda-Wert-Bestimmung}

In der Kompressionsphase eines Motors steigt zwar der Gesamtdruck und damit die Molekülzahl im Messvolumen, doch ebenso nimmt die Zahl der Stöße zwischen Molekülen in einem Gemisch mit steigendem Druck zu. Als Folge davon sinkt die Intensität einer laserinduzierten Fluoreszenz durch die Fluoreszenzlöschung, also dem strahlungslosen Energietransfer. Verschiedene Spezies, wie $\mathrm{O}_{2}, \mathrm{NO}, \mathrm{NO}_{2}$ und andere, auch bei der Verbrennung auftretende Radikale, löschen die Fluoreszenz sehr effektiv. Stickstoff, der Hauptbestandteil der Raumluft, quencht dagegen nur sehr wenig und auch andere Gase, wie $\mathrm{H}_{2} \mathrm{O}$ oder $\mathrm{CO}_{2}$ besitzen einen vernachlässigbaren Fluoreszenzlöschungseinfluss. Die Quenchingrate $Q_{21}$ ist bei hohen Drücken sehr viel größer als die Rate der spontanen Emission $A_{21}$. So ergibt sich für die Fluoreszenzintensität nach Gleichung 3.20 mit $Q_{21} \gg A_{21}$ näherungsweise:

$$
S_{f} \approx C_{1} A_{21} \frac{N}{Q_{21}} \quad \text { mit } C_{1}=B_{12} E g\left(\nu_{a b s}, \nu_{\text {laser }}\right)
$$

Wenn die Eigenfluoreszenzlöschung des Kraftstoffs oder andere Abregungsprozesse vernachlässigt werden können, hängt die Rate $Q_{21}$ nur von der Sauerstoffdichte bzw. konzentration $\left[\mathrm{O}_{2}\right]$ ab. Ebenso ist $N$ proportional zur Konzentration des fluoreszierenden Kraftstoffs [Kraftstoff]:

$$
N=k_{2}[\text { Kraftstoff }] \quad Q_{21}=k_{3}\left[\mathrm{O}_{2}\right]
$$

Aus Gleichung 3.21 folgt damit, dass das Fluoreszenzsignal proportional zum Verhältnis der Kraftstoff- zur Sauerstoffkonzentration ist:

$$
S_{f} \approx C_{1} A_{21} \frac{k_{2}[\text { Kraftstoff }]}{k_{3}\left[\mathrm{O}_{2}\right]} \quad \text { mit } \quad Q_{21} \gg A_{21}
$$




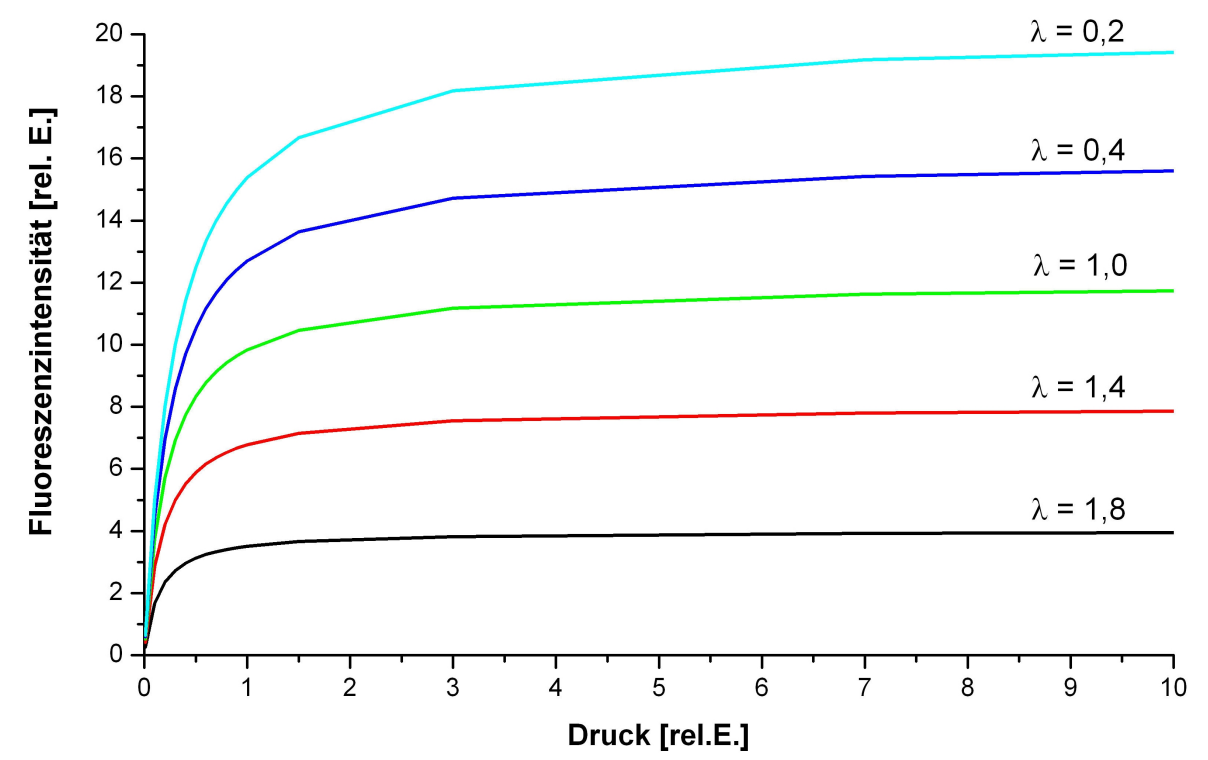

Abbildung 3.1.: Theoretische Fluoreszenzintensität bei verschiedenen Lambda-Werten in Abhängigkeit vom Druck

Wie in Kapitel 2.1 gezeigt, ist der Lambda-Wert genau dem umgekehrten Verhältnis der Konzentrationen proportional und damit ergibt sich mit der Konstante $k$ :

$$
S_{f}=k \frac{1}{\lambda} \quad b z w . \quad \lambda=k \frac{1}{S_{f}}
$$

Der Lambda-Wert ist also bei sehr hohen Drücken und damit verbundener starker Fluoreszenzlöschung durch Molekülstöße umgekehrt proportional zur Fluoreszenzintensität. Eine genaue Kalibration der Konstante $k$ ist allerdings notwendig, um Aussagen über einen Lambda-Wert treffen zu können. Eine Möglichkeit dazu ist, die Intensität mit bekannten Luft/Kraftstoffverhältnissen zu korrelieren [48].

Aus dem vorgestellten Modell ergibt sich die in Abbildung 3.1 dargestellte berechnete Abhängigkeit der laserinduzierten Fluoreszenz eines Luft/Kraftstoffgemisches vom Druck. Mit zunehmendem Druck steigt zunächst auch die Fluoreszenzintensität, da sich die Kraftstoffdichte im Messvolumen ebenfalls erhöht. Der Einfluss des Sauerstoffs auf die Fluoreszenzlöschung verstärkt sich dann aber bei hohen Drücken und führt dazu, dass die Fluoreszenz bei gleichem $\lambda$-Wert trotz steigender Kraftstoffmoleküldichte nahezu konstant bleibt [47]. Bei einem höheren Kraftstoffanteil in einem Luft/Kraftstoffgemisch, also bei einem niedrigeren $\lambda$-Wert, erhöht sich aber das Fluoreszenzsignal bei gleichem Druck.

Verschiedene Untersuchungen an Gasgemischen oder mit Fluoreszenztracern versetzten Ersatzkraftstoffen (z.B. Isooktan mit Toluol) zeigten das beschriebene Verhalten der Intensität in Abhängigkeit vom Umgebungsdruck [31, 19]. 
Bei motorischen Untersuchungen zur Bestimmung des Lambda-Wertes werden im Rahmen dieser Arbeit Raman- und LIF-Messungen kombiniert, um eine Kalibration des LIFSignals mit den aus den Raman-Messungen ermittelten $\lambda$-Werten zu ermöglichen. $\mathrm{Zu}$ späten Zeitpunkten der Kompression und daher bei hohen Zylinderinnendrücken sollte eine Korrelation der Messsignale beider Verfahren unter der Voraussetzung $Q_{21} \gg A_{21}$ und vernachlässigbarem Eigenquenching des Kraftstoffs erkennbar sein. In Abschnitt 4.3.2 wird auf das Verhalten der Fluoreszenzintensität von Realkraftstoff bei im Zuge dieser Arbeit durchgeführten motorischen Untersuchungen eingegangen. 


\subsection{Absorptionsspektroskopie}

Die Absorptionsspektroskopie ist eine der ältesten Techniken zur Analyse von Molekülgemischen. Die Lage und Intensität der Absorptionsbanden einer Substanz sind stoffspezifisch und lassen sich zur Identifizierung nutzen. Über die Stärke der Absorption kann zudem die Konzentration oder Absorberdichte in einem Gemisch sehr genau bestimmt werden. Mit Laser-Absorptionsspektroskopie ist es sogar möglich, gleichzeitig die Temperatur oder den Druck zu ermitteln. Allerdings ist es bei der Spektroskopietechnik über Absorption notwendig das zu untersuchende Gemisch zu durchstrahlen. Dadurch erfolgt immer eine integrale Messung entlang des Lichtweges.

Im Rahmen dieser Arbeit war insbesondere die Molekülabsorption von Licht im infraroten Wellenlängenbereich von Interesse. Daher wird im Folgenden genauer darauf eingegangen.

\subsubsection{Theoretische Grundlagen}

Ein Lichtquant mit der Frequenz $\nu_{n m}$ wird von einem Atom oder Molekül nur absorbiert, wenn die Photonenenergie exakt der Energiedifferenz zwischen zwei erlaubten Energieniveaus $E_{n}$ und $E_{m}$ des Moleküls entspricht, so dass

$$
h \nu_{n m}=h c \tilde{\nu}_{n m}=E_{n}-E_{m} .
$$

Dabei ist $\tilde{\nu}_{n m}$ die in der Spektroskopie üblicherweise verwendete Wellenzahl des Photons in der Einheit $\left[\mathrm{cm}^{-1}\right]$. Die Intensität der Absorption, also die von eingestrahltem Licht in einem Medium auf einer Weglänge $\Delta x$ absorbierte Energie, ist gegeben durch [24]:

$$
\Delta I\left(\nu_{n m}\right)=I_{0}\left(\nu_{n m}\right) N_{m} B_{m n} h \tilde{\nu}_{n m} \Delta x
$$

wobei $I_{0}\left(\tilde{\nu}_{n m}\right)$ die eingestrahlte Lichtintensität, $N_{m}$ die Anzahl von Molekülen im unteren Energieniveau und $B_{m n}$ der Einsteinkoeffizient der Absorption ist.

Das Spektrum von Übergängen zwischen Schwingungs-Niveaus innerhalb desselben elektronischen Zustandes in einem Molekül liegt im infraroten Spektralbereich. Eine Interaktion von eingestrahltem infrarotem Licht mit einem vibrierenden Molekül ist allerdings nur möglich, wenn das äußere elektrische Feld mit der selben Frequenz wie das Dipolmoment des Moleküls schwingt. Wie bereits in Abschnitt 3.1.1 beschrieben, führt das elektrische Feld des Lichtes beim Auftreffen auf dem Molekül zu einem zeitabhängigen Dipolmoment $\vec{p}$. Das Molekül wird als Infrarot aktiv bezeichnet, wenn dieses Dipolmoment von der Auslenkung der Kerne bestimmt wird und

$$
\left(\frac{\partial \vec{p}}{\partial Q_{n}}\right)_{0} \neq 0
$$

in Gleichung 3.6 gilt, also ein permantentes Dipolmoment vorhanden ist. Ist kein Dipolmoment von vornherein vorhanden, so können zumindest Schwingungen angeregt werden, 
bei denen durch asymmetrische Verlagerung der Ladungsschwerpunkte ein Dipolmoment entsteht. Bei einigen Schwingungen erfolgt keine Dipolmomentänderung und man bezeichnet diese als Infrarot inaktiv. Ein Beispiel dafür ist die symmetrische Schwingung der Kohlendioxidmoleküle [21].

Bei homonuklearen Molekülen, wie z.B. $\mathrm{O}_{2}$ oder $\mathrm{N}_{2}$, besitzen die atomaren Bausteine gleiche Kernladungszahl und damit ist $\left(\partial \vec{p} / \partial Q_{n}\right)=0$. Sie können daher prinzipiell nicht durch IR-Strahlung zu Schwingungen angeregt werden, besitzen also kein IR-Spektrum.

Quantenmechanisch sind nur Übergänge zwischen erlaubten Vibrations- oder Rotationsniveaus möglich. Dabei gelten folgende Auswahlregeln:

$$
\begin{aligned}
& \Delta v=0 \quad \Delta J= \pm 1 \quad \text { für reine Rotationsanregung und } \\
& \Delta v= \pm 1,2,3 \ldots \quad \Delta J= \pm 1 \quad \text { für Vibrations-Rotations-Übergänge }
\end{aligned}
$$

Daraus folgt, dass Schwingungsübergange zwischen beliebigen Schwingungsniveaus stattfinden können, der damit verbundene Rotationsquantensprung aber nur in den nächsthöheren oder nächstniedrigeren Energiezustand erfolgt. Reine Schwingungsübergänge mit $\Delta J=0$ sind also verboten. In einem Rotationsschwingungsspektrum wird die Linienserie mit $\Delta J=+1$ als R-Zweig, die mit $\Delta J=-1$ als P-Zweig bezeichnet.

Für homogen verteilte Absorbermoleküle im Gemisch ergibt sich aus der Integration von Gleichung 3.26 allgemeiner das Lambert-Beer-Gesetz für die Absorption:

$$
I(\lambda)=I_{0}(\lambda) e^{-\sigma_{\lambda} C L}
$$

mit der Absorberkonzentration C (g/l, mol/l, o.ä.), dem Absorptionsweg L und der eingestrahlten Intensität $I_{0}$. Der lineare Absorptionskoeffizient $\sigma_{\lambda}$ in den entsprechenden Einheiten der Konzentration ist abhängig von der Wellenlänge.

\subsubsection{Linienstärke und -profil von Absorptionslinien}

Der Absorptionskoeffizient $\sigma$ lässt sich schreiben als ein Produkt aus wellenlängenunabhängiger Linienstärke $S$ und normiertem Linienprofil $g\left(\tilde{\nu}-\tilde{\nu}_{0}\right)\left[1 / \mathrm{cm}^{-1}\right]$ mit Halbwertsbreite $\gamma\left[\mathrm{cm}^{-1}\right]$ und Mittenwellenzahl $\tilde{\nu}_{0}$ als [62]:

$$
\begin{gathered}
\sigma(\tilde{\nu})=S g\left(\tilde{\nu}-\tilde{\nu}_{0}\right), \quad \int_{-\infty}^{\infty} g\left(\tilde{\nu}-\tilde{\nu}_{0}\right) d \nu=1 \\
S=\int_{-\infty}^{\infty} \sigma(\tilde{\nu}) d \tilde{\nu}
\end{gathered}
$$

Die Linienstärke $S$ entspricht also dem Integral über die gesamte spektrale Breite der Absorptionslinie. Mit der Temperatur ändert sich die Besetzung der einzelnen Energieniveaus. Dies führt dazu, dass $S$ stark temperaturabhängig ist. Eine Temperaturänderung von einigen hundert Grad kann die Größe der Linienstärke um zwei Größenordnungen ändern. 


\subsubsection{Natürliche Linienbreite}

Bei einem ruhendem Atom oder Molekül hängt die spektrale Breite einer Absorptionslinie von der Lebensdauer der beiden am Übergang beteiligten Niveaus ab. Die mittlere Lebensdauer eines Zustandes $\tau_{i}=1 / A_{i}$ wird bestimmt durch den Einstein-Koeffizient $A_{i}$ der spontanen Emission:

$$
\gamma_{N}=A_{i}=\frac{1}{\tau_{i}}
$$

Dabei ist $\gamma_{N}\left[1 / \mathrm{cm}^{-1}\right]$ die Halbwertsbreite der Absorptionslinie. Dies gilt ebenso für die Emission. Durch spontane Prozesse können elektromagnetische Wellen vom Atom oder Molekül absorbiert bzw. emittiert werden. Beschreiben lässt sich dies mit Hilfe des klassischen Modells eines harmonischen Oszillators. Daraus ergibt sich, dass die Form der natürlichen Absorptions- bzw. Emissionslinie von einem Lorentz-Profil beschrieben wird $[52]$ :

$$
g_{\text {Lorentz }}\left(\tilde{\nu}-\tilde{\nu}_{0}\right)=\frac{1}{\pi} \frac{\gamma}{\left(\tilde{\nu}-\tilde{\nu}_{0}\right)^{2}+\gamma^{2}}
$$

Ist die Lebensdauer eines Übergangs groß, so wird dessen natürliche Linienbreite sehr schmal.

\subsubsection{Dopplerverbreiterung}

Auch ein bewegtes Atom oder Molekül emittiert bzw. absorbiert Licht bei einem Übergang zwischen zwei Niveaus immer mit seiner natürlichen Linienbreite. Doch die Wellenlänge dieses Lichts wird für einen ruhenden Beobachter aufgrund seiner relativen Geschwindigkeit zur Lichtquelle dopplerverschoben. Aus der maxwellschen Geschwindigkeitsverteilung von Atomen im thermischen Gleichgewicht ergibt sich dann als normierte dopplerverbreiterte Linienform bei niedrigen Drücken das Gauss-Profil:

$$
g_{\text {Doppler }}\left(\tilde{\nu}-\tilde{\nu}_{0}\right)=\sqrt{\frac{4 \ln 2}{\pi}} \frac{1}{\gamma_{D}} \exp \left[-4 \ln 2\left(\frac{\tilde{\nu}-\tilde{\nu}_{0}}{\gamma_{D}}\right)^{2}\right]
$$

mit der Mittelwellenzahl $\tilde{\nu}_{0}$. Die Halbwertsbreite $\gamma_{D}\left[1 / \mathrm{cm}^{-1}\right]$ hängt von der Masse der Moleküle und der Temperatur ab:

$$
\begin{aligned}
\gamma_{D} & =\frac{\tilde{\nu}_{0}}{c} \sqrt{\frac{8 k_{B} T \ln 2}{M}} \\
& =7,1623 \cdot 10^{-7} \tilde{\nu}_{0} \sqrt{\frac{T}{M}}
\end{aligned}
$$

mit der Boltzmann-Konstate $k_{B}$, der Temperatur in $[\mathrm{K}]$ und der Masse des Moleküls in atomaren Masseneinheiten. 


\subsubsection{Druckverbreiterung}

In einem Gas führen bei Drücken oberhalb von 1bar Stöße zwischen den Molekülen vermehrt zu einer Verkürzung der Lebensdauer von angeregten Zuständen und damit nach Gleichung 3.31 zu verbreiterten Linien. Neben der Linienverbreiterung tritt allerdings auch noch eine geringe Verschiebung der Mittenwellenzahl auf. Die Linienform wird ebenso wie bei der natürlichen Linienbreite durch das Lorentz-Profil (Gleichung 3.32) beschrieben. Dabei hängt die Halbwertsbreite $\gamma(p, T)\left[\mathrm{cm}^{-1}\right]$ ab von der durch Fremdgas hervorgerufenen Linienverbreiterung $\gamma_{f}$ und der durch Moleküle der selben Spezies verursachten Verbreiterung $\gamma_{s}$ nach:

$$
\gamma(p, T)=\left(\frac{T_{0}}{T}\right)^{n}\left(\gamma_{f} \frac{p-p_{s}}{p_{0}}+\gamma_{s} \frac{p_{s}}{p_{0}}\right)
$$

Dabei sind $\gamma_{f}$ und $\gamma_{s}$ die Linienverbreiterungen in $\left[\mathrm{cm}^{-1} / \mathrm{bar}\right]$ bei $T_{0}=296 \mathrm{~K}$ und $p_{0}=1013 \mathrm{mbar}$. Der Faktor $n$ beschreibt die Temperatur-Abhängigkeit der Verbreiterung und ist für jede Molekülspezie verschieden. Beispielsweise ist $n=0,71$ für $\mathrm{O}_{2}$ und $n=0,64$ für $\mathrm{CO}_{2}[49]$.

Liegen Druck- und Temperaturbedingungen vor, so dass es sowohl zu einer Druck- als auch einer Dopplerverbreiterung der Linie kommt, so lässt sich die Linienform durch ein Voigt-Profil beschreiben. Dies ist eine Faltung eines Lorentz- und eines Gauss-Profils. 


\subsubsection{Lambda-Wert-Bestimmung}

Mit Hilfe der Absorptionsspektroskopie lassen sich die Konzentrationen von Molekülspezies in einem Gemisch sehr gut bestimmen, wenn deren Absorptionsbanden im betrachteten Wellenlängenbereich nicht überlappen. Für die Bestimmung des Lambda-Wertes eines Luft/Kraftstoffgemisches ist es daher notwendig, solche Wellenlängenbereiche für Untersuchungen auszuwählen, in denen sich Sauerstoff- und Kraftstoffkonzentrationen ohne Interferenzen anderer Spezies eindeutig bestimmen lassen.

Die in Kraftstoff enthaltenen Kohlenwasserstoffe sind infrarot aktive Moleküle und besitzen sehr starke Absorptionsbanden im mittleren infraroten Spektralbereich. Beispielhaft sind die verschiedenen Banden von Methan in diesem Wellenlängenbereich in Abbildung 3.2 dargestellt. Berechnet wurde dieses Absorptionssektrum mit Hilfe der HITRAN database [49]. Die stärkste Absorptionsbande der Kohlenwasserstoffe um 3,4 $\mu$ m resultiert aus der CH-Streckschwingung. Der erste Oberton dieser Schwingung liegt um 1,66 $\mu \mathrm{m}$. Kombinationsschwingungen sind die Ursache der anderen beiden Banden. In einem Motor können nur kurze Absorptionsstrecken bei der Bestimmung der Kraftstoffkonzentration am Ort der Zündkerze generiert werden. Daher erfolgen die Untersuchungen im Rahmen dieser Arbeit im Wellenlängenbereich der CH-Grundschwingung um 3,4 $\mu \mathrm{m}\left(=2941 \mathrm{~cm}^{-1}\right)$. Die Absorption von $\mathrm{H}_{2} \mathrm{O}$ bei dieser Wellenlänge ist um etwa einen Faktor 100 schwächer als die der Kohlenwasserstoffe [49], und weitere Absorptionsbanden anderer Molekülspezi-

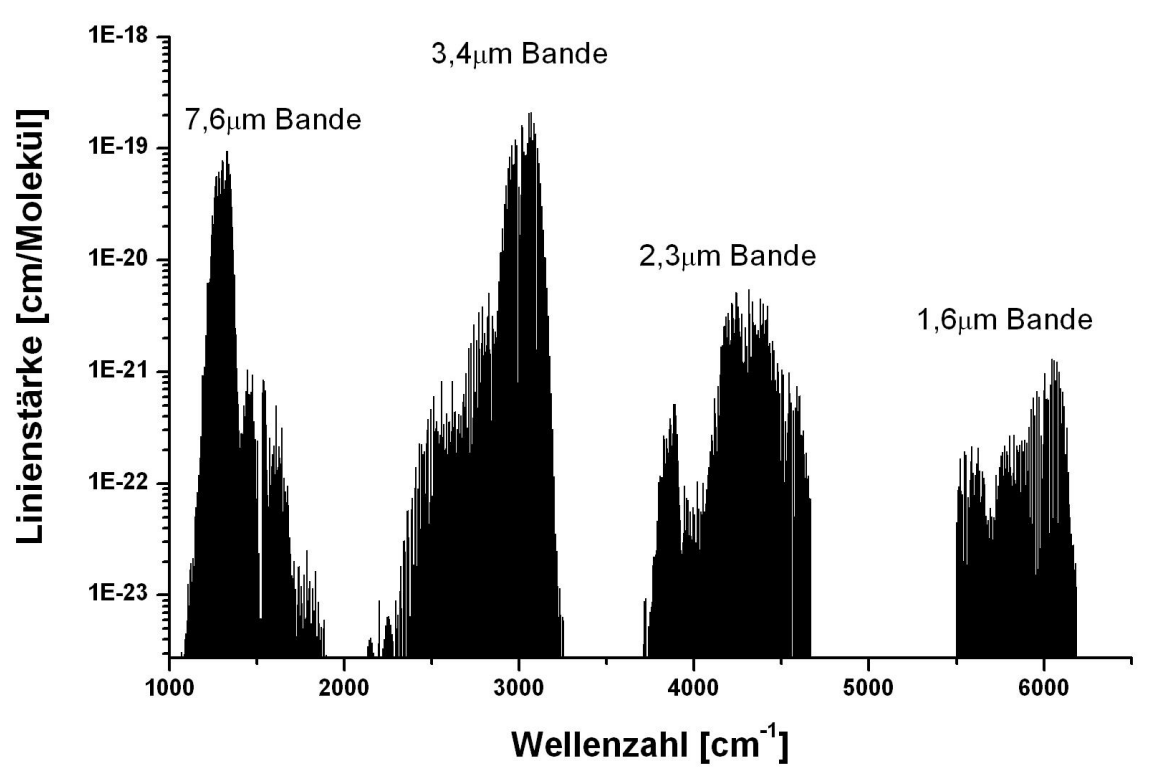

Abbildung 3.2.: Absorptionslinien von Methan (berechnet mit HITRAN database [49]) 
es eines Luft/Kraftstoffgemischs in diesem Spektralbereich existieren nicht, so dass immer eine eindeutige Zuordnung zur Kraftstoffkonzentration möglich ist.

Sauerstoff dagegen ist als homonukleares Molekül Infrarot inaktiv und besitzt daher keine Absorptionsbanden in diesem Wellenlängenbereich. Die stärkste Absorptionsbande der $\mathrm{O}_{2}$-Moleküle im sichtbaren Spektralbereich ist der elektronisch dipolverbotene Übergang $b^{1} \Sigma_{g}^{+}\left(v^{\prime}=0\right)-X^{3} \Sigma_{g}^{-}\left(v^{\prime \prime}=0\right)$ um $760 \mathrm{~nm}$ mit einer Linienstärke um $5 \cdot 10^{-24} \mathrm{~cm} /$ Molekül bei Raumtemperatur [49]. Damit ist die Absorption um etwa fünf Größenordnungen schwächer als die der Kohlenwasserstoffmoleküle um 3,4 $\mu \mathrm{m}$.

Anders als bei der Raman-Spektroskopie ist es also bei der Absorptionsspektroskopie für die Bestimmung der Sauerstoff- und Kraftstoffkonzentration notwendig Lichtquellen mit unterschiedlichen Wellenlängen in getrennten Detektionssystemen einzusetzen. Werden beide Systeme, um die jeweilige Konzentration zu bestimmen, einzeln unter Verwendung von Gleichung 3.29 kalibriert, so kann anschließend mit Hilfe der Gleichung 2.1 der $\lambda$-Wert von Luft/Kraftstoffgemischen bestimmt werden.

Im Rahmen dieser Arbeit wurden Messsysteme für beide Spezies realisiert. Mit dem Detektionssystem zur Bestimmung der Kraftstoffkonzentration mittels InfrarotAbsorptionsspektroskopie wurden Untersuchungen am Ottomotor durchgeführt (Abschnitt 5.1). 


\section{Raman-spektroskopische Gemischbildungsanalyse in Ottomotoren}

In Ottomotoren wurde in der Vergangenheit die quantitative Bestimmung des $\lambda$-Wertes mittels spontaner Raman-Streuung auf Grund der höheren Raman-Streuquerschnitte üblicherweise mit ultravioletter Laserstrahlung durchgeführt [53, 32]. Da allerdings mit UV-Licht angeregter Realkraftstoff durch die in ihm enthaltenen aromatischen Anteile unerwünschte Fluoreszenzen zeigt und diese das schwächere Raman-Signal überlagern, konnten Untersuchungen bislang nur mit aromatenfreien Ersatzkraftstoffen durchgeführt werden. Als Ersatzkraftstoffe wurden beispielsweise Einkomponentenkraftstoffe wie Isooktan, Alkohole (Methanol, Ethanol) oder alkanische Mehrkomponentenkraftstoffe (MKK) verwendet [40]. Neuere Untersuchungen zeigen jedoch das unterschiedliche Verhalten von Ersatzkraftstoffen und Realkraftstoffen, wie z.B. Euro Super, bei der Gemischbildung und Sprayausbreitung in Ottomotoren [35]. Daher ist für eine Optimierung von benzindirekteinspritzenden Brennverfahren die Analyse der realen innermotorischen Vorgänge durch ein hoch zeitaufgelöstes Messverfahren an Realkraftstoffen unerlässlich.

Da der $\lambda$-Wert nur für den gasförmigen Zustand definiert ist, ergeben sich weitere Probleme bei dessen exakter Bestimmung mit Hilfe der spontanen Raman-Streuung, wenn Kraftstofftröpfchen im Messvolumen vorhanden sind. Zudem erschwert eine besondere Art der Wechselwirkung kleiner transparenter Tröpfchen mit Laserstrahlung hoher Leistung 
die Analyse: Einerseits kommt es zu einer starken Fokussierung des hochenergetischen Laserlichtes und damit zur Generierung von Gasdurchbrüchen mit sehr hellen, die schwachen Ramanlinien vollständig überlagernden Emissionen. Andererseits führt die in Tröpfchen mit ihrer hohen Dichte erzeugte extrem starke, zum Teil stimulierte Raman-Streuung zu einer Verfälschung der $\lambda$-Wert-Messung. Somit war es notwendig, eine Messtechnik zu entwickeln, die auch unter diesen Bedingungen erfolgreich einsetzbar ist, da in Ottomotoren sogar noch bis zum Zeitpunkt der Zündung Kraftstofftröpfchen vorkommen.

In diesem Kapitel soll zunächst ein Detektionssystem vorgestellt werden, mit dem es erstmals möglich ist, Raman-spektroskopische Messungen des $\lambda$-Wertes in tröpfchenbeladenen Realkraftstoffsprays durchzuführen. Um interferierende Fluoreszenzemissionen von aromatischen Kohlenwasserstoffkomponenten zu vermeiden, wird dabei Laserstrahlung im sichtbaren Spektralbereich verwendet. Zudem kann durch die Zugabe eines nicht verdampfenden Absorberfarbstoffes die starken Raman-Emissionen unterdrückt werden. Der erfolgreiche Einsatz dieses Messsystems für ortsaufgelöste Messungen des Luft/KraftstoffVerhältnisses an einem Transparentmotor wird gezeigt.

Diese Erkenntnisse bilden die Grundlage für die Entwicklung eines faseroptischen Sensorsystems zur Bestimmung des $\lambda$-Wertes, welches anstatt der Zündkerze in einem Ottomotor eingesetzt wird. Im Weiteren wird in diesem Kapitel der experimentelle Aufbau und die Anforderungen an ein solches System beschrieben und detailliert die Möglichkeiten und Grenzen eines faseroptischen Detektionssystems erläutert.

\subsection{Komponenten des Detektionssystems}

\subsubsection{Lasersystem}

Der für die Raman-Experimente verwendete Laser war ein Blitzlampen gepumpter Quantel Brilliant B Nd:YAG-Laser, der auf eine Wellenlänge von 532nm frequenzverdoppelt wurde. Die Pulsdauer betrug 5ns mit einer Pulsenergie von bis zu 250mJ. Der Laserstrahl war stark polarisiert und besaß ein Gauß-Profil mit einem Durchmesser von $8 \mathrm{~mm}$. Allerdings enthielt das Laserstrahlprofil einige „Hot Spots“, also Bereiche sehr hoher Leistungsdichte. Dieses führte zu einigen Problemen bei dem Versuch, das Laserlicht zu fokussieren, um eine möglichst hohe Laserenergiedichte in einem Messvolumen oder zur Einkopplung in eine Glasfaser zu erhalten. So geschah es beispielsweise gelegentlich, dass Frontseiten von Glasfasern beim Einkoppeln des Laserlichtes zerstört wurden. Dieses hatte einen großen Einfluss auf die Wahl der Glasfasern und die letztendlich realisierte faseroptische Sensorkopfgeometrie (siehe Abschnitt 4.4).

Zudem bestand die Gefahr der Zerstörung des Quarzglases von optischen Zugängen in Druckkammern oder Transparentmotoren. Insbesondere bei Transparentmotoren, also modifizierten Ottomotoren, bei denen ein Teil des Motorzylinders aus Glas besteht, stellt 
dies durch den hohen Zylinderinnendruck von mehr als 10bar und damit der Gefahr des Berstens des Glases ein großes Sicherheitsrisiko dar.

Ein weiteres Problem bestand darin, dass es auf Grund der hohen Feldstärken in den „Hot Spots“ des fokussierten Laserstrahls durch die Ionisation von Luftmolekülen zu Plasmazündungen kam. Die Schwelle für Luftdurchbrüche in reiner partikelfreier Luft bei einer Laserwellenlänge von $532 \mathrm{~nm}$ liegt bei ca. $10^{11} \mathrm{~W} / \mathrm{cm}^{2}[44,58]$. Die Rekombination der Elektronen im Plasma führt zu einer breitbandigen Emission, welche stärker ist als das Raman-Streulicht. Bei hohen Drücken, wie sie bei der Kompression im Motor entstehen, sinkt die Durchbruchschwelle. Sie ist näherungsweise umgekehrt proportional zum Druck und damit zur Dichte [15]. Der genaue Schwellwert hängt allerdings sehr stark von den Randbedingungen, wie z.B. Oberflächen des Brennraumes, ab [61].

Um die Bereiche hoher Leistungsdichte im Laserstrahlprofil zu minimieren, wurde ein Strahlhomogenisator (siehe Abbildung 4.1) eingesetzt. Der Laserstrahl wurde dafür zunächst durch ein Galileo-Teleskop fokusfrei aufgeweitet und durchlief dann den Homogenisator [51]. Dieser besteht aus zwei direkt hintereinander liegenden um $90^{\circ}$ gekreuzten Zylinderlinsenfeldern aus je sieben Linsen, welche eine Brennweite von $800 \mathrm{~mm}$ und eine Größe von $50 \mathrm{~mm} \times 4 \mathrm{~mm}$ besitzen. Anschließend wurden die dadurch entstandenen Einzelstrahlen durch eine Sammellinse fokussiert.

Die so entstandenen 49 Einzelfoki besaßen nicht die Leistungsdichten um Luftdurchbrüche zu erzeugen. In der dahinterliegenden Strahltaille werden die Teilstrahlen des Homogenisators überlagert und erzeugen so ein gemeinsames, quadratisches Abbild. Ihr Durchmesser hängt von der Brennweite der Sammel- und Zylinderlinsen und deren Breite ab:

$$
S=\frac{d f_{\text {sammel }}}{f_{z y l}}
$$

mit

$$
\begin{aligned}
S & : \text { Durchmesser der Fokustaille } \\
d & : \text { Breite der Zylinderlinsen } \\
f_{\text {sammel }} & : \text { Brennweite der Sammellinse } \\
f_{z y l} & : \text { Brennweite der Zylinderlinsen }
\end{aligned}
$$

Die Brennweite der Sammellinse wurde jeweils den verschiedenen Bedingungen der Experimente angepasst. Im faseroptischen Aufbau bestimmte die numerische Apertur (NA) der verwendeten Glasfaser die Wahl der Linse. Dagegen war es bei den Freistrahlexperimenten am Transparentmotor wichtig, dass die Vorfoki außerhalb des Glasringes lagen, um die Möglichkeit für dessen Beschädigung durch Plasmadurchbrüche im Glas auszuschließen. 


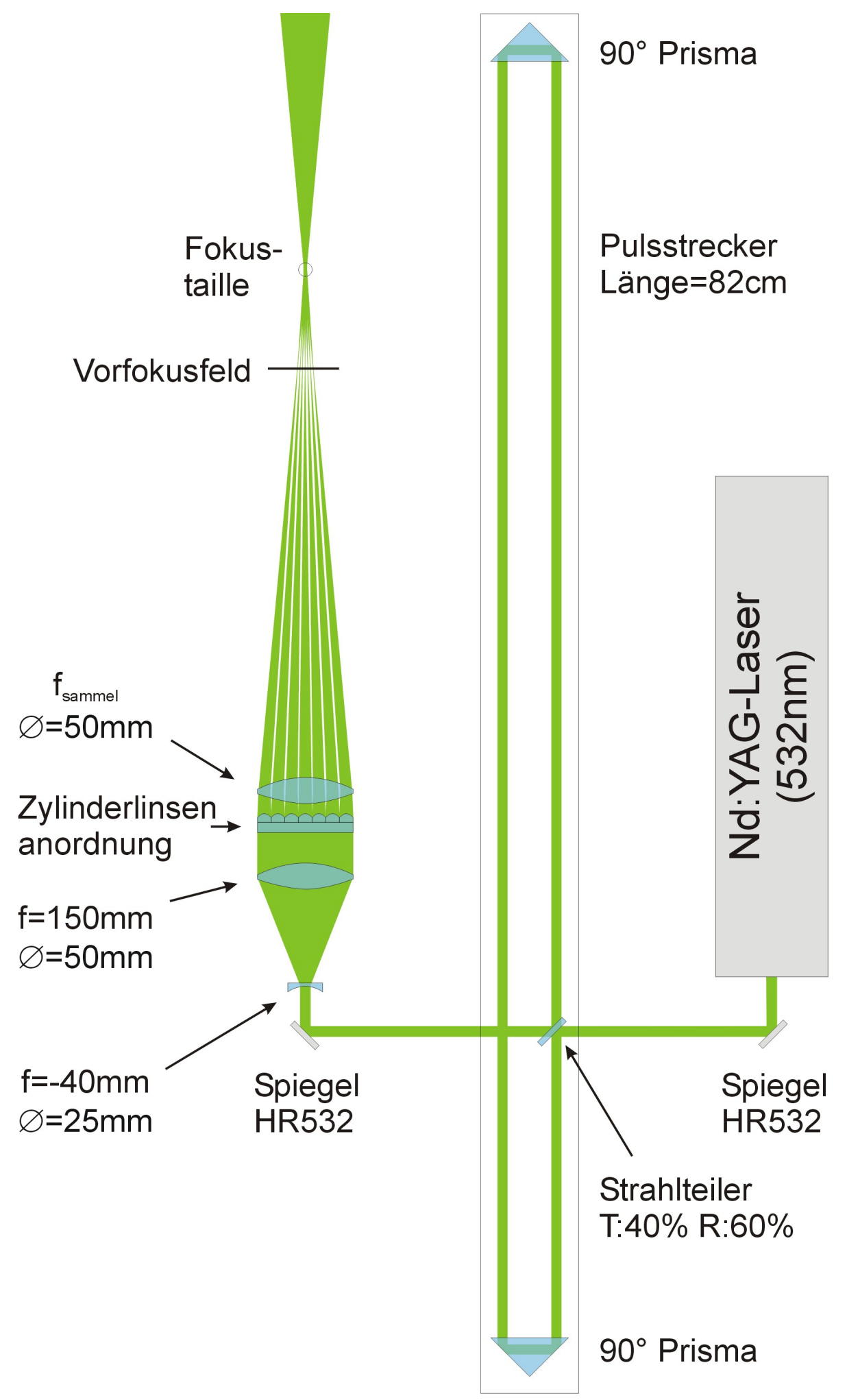

Abbildung 4.1.: Aufbau des Lasersystems mit Strahlhomogenisator und Pulsstrecker 


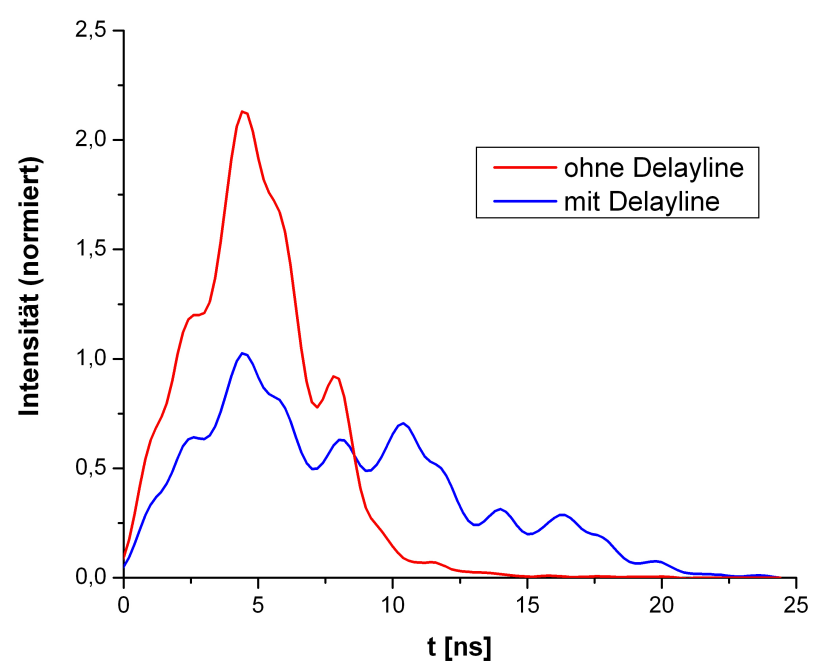

Abbildung 4.2.: Zeitlicher Laserpulsverlauf mit und ohne Delayline

Durch die Dauer des Laserpulses erhält man die Zeitaufösung der mit der spontanen Raman-Streuung beobachteten Vorgänge. Sie liegt mit 5ns weit unter der, die man zur Analyse der innermotorischen Gemischbildungsprozesse benötigt. Daher bestand eine weitere Möglichkeit die hohen Leistungsdichten zu verringern darin, den Laserpuls durch einen Pulsstrecker zeitlich zu strecken. Da die Frequenzverdopplung der Fundamentalwellenlänge des Nd:YAG-Lasers (1064nm) um so effektiver ist, je kürzer der Laserpuls und damit die Leistungsdichte ist, konnte die Pulsstreckung nur danach erfolgen.

Der Pulsstrecker bestand aus einem Strahlteiler und zwei $90^{\circ}$-Präzisionsprismen, die als Verzögerungsstrecke angeordnet waren (Abbildung 4.1). Bei diesem Aufbau wird der einlaufende Laserpuls in viele Teilpulse zerlegt, die zeitlich, durch die Länge der Verzögerungsstrecke vorgegeben, hintereinander angeordnet werden. Das Strahlteilerverhältnis und die Streckenlänge wurden durch ein Simulationsprogramm bestimmt [50], welches eine Variation der Prismenabstände, Strahlteilerverhältnisse und Umlaufverluste erlaubt. Als optimale Konfiguration, um die Spitzenintensität zu minimieren und den Puls zusammenhängend zu strecken, ergab sich ein Prismenabstand von $82 \mathrm{~cm}$ und ein Strahlteilerverhältnis von 60\% Reflektion und 40\% Transmission. Das experimentelle Ergebnis für den gestreckten Laserpuls ist in Abbildung 4.2 dargestellt. Dabei wurden die Pulse mit einem Photomultiplier, welcher eine hohe Zeitauflösung ermöglicht, und einem 500MHz-Speicheroszilloskop aufgezeichnet und über 128 Pulse gemittelt. Die Spitzenintensität konnte so halbiert werden bei ca. 15\% Verlusten durch Oberflächenreflexe.

Durch die Verwendung des Homogenisators und des Pulsstreckers konnte sowohl eine Zerstörung des Glasringes, als auch eine Fehlzündung des hochkomprimierten Luft/Kraftstoffgemisches im Zylinder des Transparentmotors vermieden werden. 


\subsubsection{Signaldetektion}

Die Detektion des durch die Anregung mit dem Nd:YAG-Laser entstandenen RamanStreulichts erfolgte durch einen optischen Mehrkanal-Analysator (OMA). Eingesetzt wurde hierfür ein ortsauflösender Gitterspektrograph (Acton Research - „SpectraPro 300i“) mit einem $14 \mathrm{~mm}$ langen Eintrittsspalt und einer f-Zahl von 3,8. Das verwendete 600Linien/mm Gitter besitzt eine Blazewellenlänge von 500nm. Um eine Überstrahlung des Spektrographen durch Rayleigh- oder Mie-gestreutes Licht zu verhindern, wurde vor dem Eintrittsspalt eine Filterkombination aus einem Farbfilter (Schott - OG550) und einem Notchfilter mit $<20 \mathrm{~nm}$ Bandbreite (Kaiser Optik) zur Unterdrückung der Anregungswellenlänge 532nm plaziert.

Für nichtortsaufgelöste Messungen wurde zur spektralen Selektion eine zweite Spaltblende am Ausgang des Spektrographen installiert und das transmittierte Licht mit einem Channel Photomultiplier (CPM) der Firma PerkinElmer (Typ MH-972P) detektiert. Dieser besaß einen sehr niedrigen Dunkelstrom und war mit einem Verstärkungsfaktor von bis zu $10^{8}$ auf Einzelphotonenzählen ausgelegt.

Sollte dagegen die Ortsinformation erhalten bleiben, konnte das Spektrum mit einer intensivierten slow scan CCD-Kamera (LaVision - „Flamestar III“) aufgenommen werden. Der vor dem eigentlichen CCD-Chip der Kamera befindliche hochspannungsbetriebene Bildverstärker erlaubt es zum einen die Belichtungszeit des CCD-Chips zu reduzieren, um so weniger Hintergrundstrahlung aufzunehmen, zum anderen das einfallende Signal zu verstärken und so das Signal zu Rauschverhältnis zu verbessern. Zudem war die Kamera mit einer sog. Pixel-Binning-Funktion ausgestattet. Damit war es möglich auf dem CCD-Chip einzelne Pixel einer Spalte vor dem Auslesen durch Ladungsverschiebung zu Superpixeln zusammenzufassen, um so das Ausleserauschen zu vermindern . Die Kamera wurde so orientiert, dass die Aufösung der spektralen Achse erhalten blieb. Obwohl auf diese Weise die örtliche Auflösung reduziert wird, können so durch die Erhöhung des Signal/Rausch-Verhältnisses auch noch sehr schwache Signale detektiert werden. Bei allen Untersuchungen wurde ein Binning-Faktor von 8 gewählt, das heißt, es werden acht Pixel zu einem Superpixel zusammengefasst. Bei einer Auflösung des CCD-Chips von 1024x1024 Pixel führt dies zu 128 Superpixeln auf der Ortsachse und damit bei 14mm Messbereich zu einer Ortsauflösung von 0,1mm. Diese war für die Analyse der örtlichen Verteilung des Luft/Kraftstoffgemisches ausreichend. 


\subsection{Voruntersuchungen}

Zunächst wurde eine Druckkammer für erste Voruntersuchungen konstruiert, welche den Abmessungen des Transparentmotors, der für Untersuchungen realer motorischer Vorgänge zur Verfügung stand, entsprach. Insbesondere die Position und Dicke der Fenster waren dem Glasring des Motors nachempfunden. Die Druckkammer war sowohl vakuum- als auch überdrucktauglich bis 3bar und konnte mittels einer Drehschieberpumpe bis auf etwa $10^{-4}$ bar evakuiert werden. Um in der Kammer definierte homogene Luft-Kraftstoffgemische untersuchen zu können, konnte diese mit Hilfe eines beheizbaren Vormischbehälters befüllt werden. Dabei wurde der Kammerinnendruck mit einer Genauigkeit von 0,2mbar bestimmt. Zur Untersuchung des Verhaltens eines Kraftstoffsprays konnte in die Kammer ein BDE-Injektor (Firma Bosch) eingebaut werden. Der experimentelle Aufbau der Kammeruntersuchungen ist in Abbildung 4.3 dargestellt.

Der durch den Homogenisator von „Hot Spots“ befreite und durch den Pulsstrecker zeitlich gedehnte Laserpuls des Nd:YAG-Lasers wurde mit einer langbrennweitigen Linse $\left(f_{\text {sammel }}=400 \mathrm{~mm}\right)$ in die Druckkammer fokussiert. Dabei war die Brennweite so gewählt, dass die Vorfoki außerhalb der Kammer und daher auch außerhalb des Glasringes des Transparentmotors lagen. Dadurch entstand ein langer Fokus mit einer $2 \mathrm{~mm} \times 2 \mathrm{~mm}$ großen Strahltaille. Das Messvolumen in der Kammer wurde senkrecht zum Laserstrahl f-Zahl angepasst auf den Spektrographenspalt abgebildet.
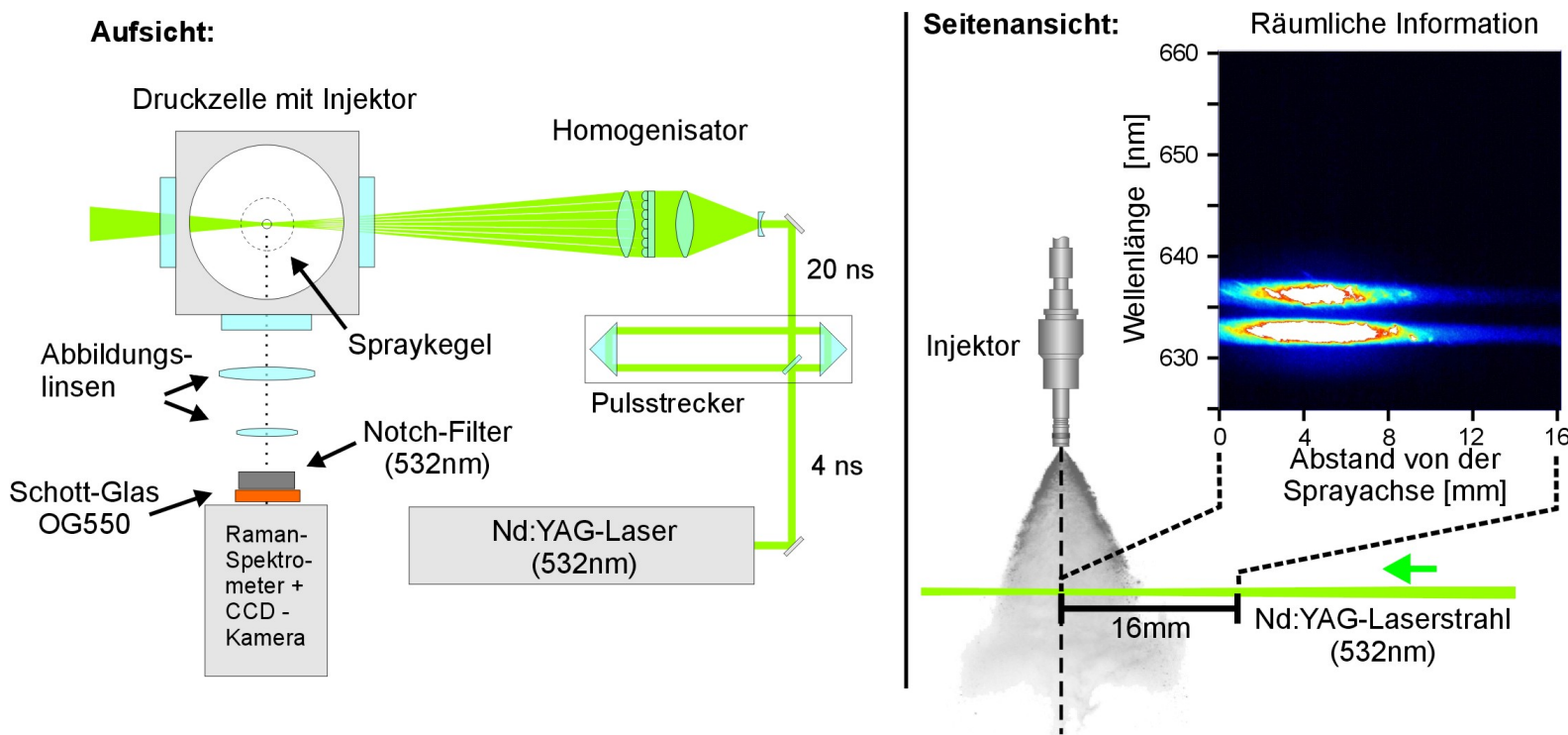

Abbildung 4.3.: Experimenteller Aufbau für Raman-spektroskopische Experimente in einer Druckkammer. Eine eingebaute Einspritzdüse ermöglichte Untersuchungen an Kraftstoffsprays. 


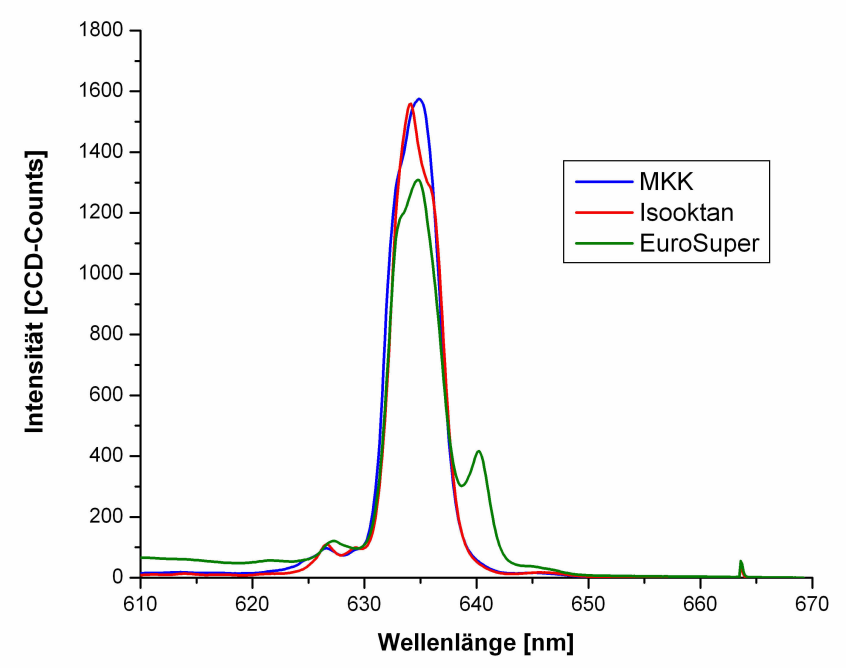

Abbildung 4.4.: Raman-Spektren verschiedener flüssiger Kraftstoffe

Aus Sicherheitsgründen wurde bei allen Untersuchungen keine Luft, sondern Stickstoff verwendet und die Kammer bei Sprayexperimenten Stickstoff gespült betrieben, um eine unbeabsichtigte Zündung des Luft-Kraftstoffgemisches zu verhindern. Da die Kammer nur eine Druckfestigkeit von etwa 3bar besitzt, würde sie dementsprechend einem Explosionsdruck nicht standhalten. Durch das feste Verhältnis von Sauerstoff zu Stickstoff in Raumluft kann immer auf den tatsächlichen Lambda-Wert des Gemisches zurück gerechnet werden.

\subsubsection{Auswahl des Kraftstoffes}

Um eine Analyse des Gemischbildungsprozesses bei Bezindirekteinspritzung mit einer Laserwellenlänge von 532nm durchführen zu können, wurden zunächst Raman-Spektren von flüssigen Modell- und Realkraftstoffen in einer Küvette untersucht. Dazu wurde eine Küvette im Laserfokus plaziert und mit verschiedenen Kraftstoffen befüllt. In Abbildung 4.4 sind die aus 500 Einzelschüssen gemittelten Raman-Spektren von MKK, Isookten und Euro Super gezeigt. Die Spaltbreite des Spektrometerspaltes betrug 0,5mm. Das detektierte Signal bei etwa $663 \mathrm{~nm}$ ist auf einen Fehler des CCD-Chips der Kamera zurückzuführen.

Im Gegensatz zu den Experimenten mit UV-Licht-Anregung waren die durch die Aromaten im Euro Super verursachten breitbandigen Fluoreszenzemissionen sehr klein. Deutlich zu sehen ist die Vibrations-Ramanlinie der CH-Bindungen aller drei Kraftstoffe bei etwa 635nm. Bei Euro Super allerdings, welcher als einziger Kraftstoff aromatische Bestandteile (z.B. Benzol) enthält, taucht ein zusätzlicher Peak bei etwa 640nm auf. In 

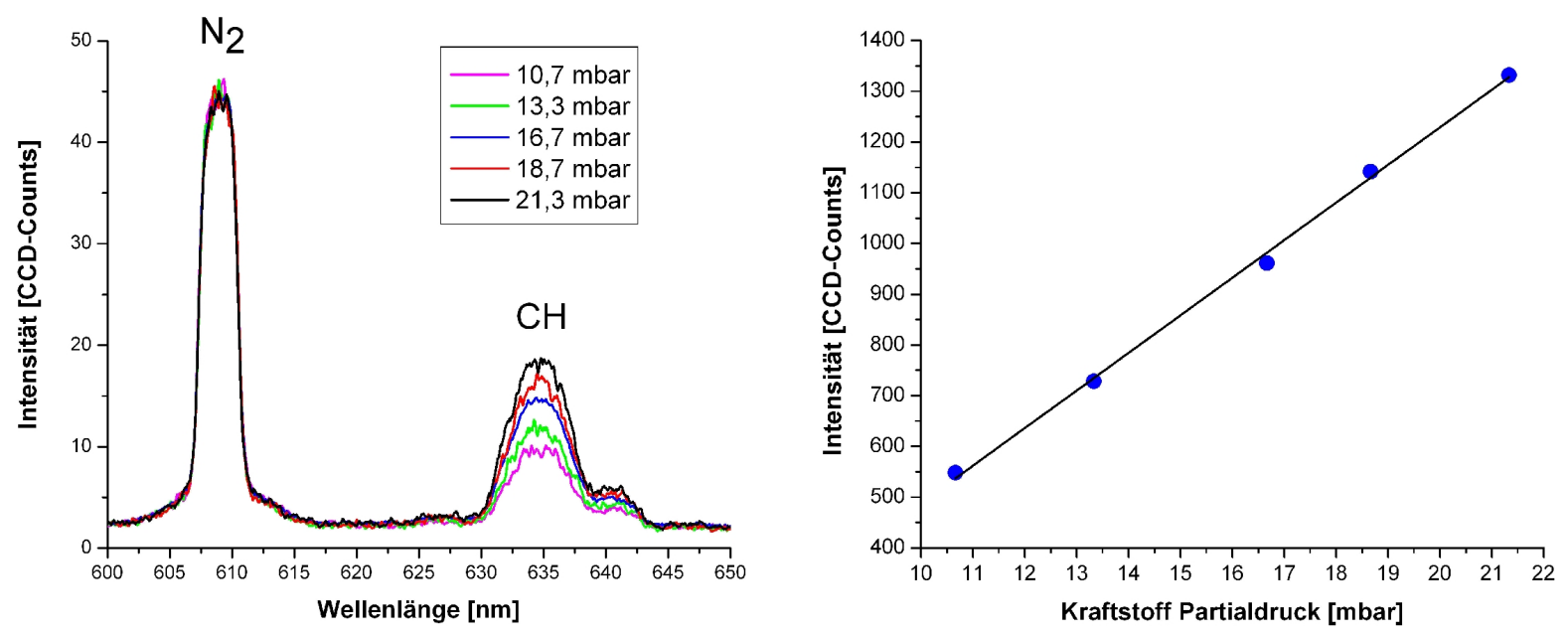

Abbildung 4.5.: a) Raman-Spektren verschiedener Euro Super/Stickstoff-Gemische mit konstantem Gesamtdruck (1bar) und unterschiedlichem Partialdruck von Euro Super b) Das untergrundkorrigierte CH-Signal zeigt eine lineare Abhängigkeit zum Kraftstoff-Partialdruck

ungesättigten Kohlenwasserstoffen ist die Elektronegativität des Kohlenstoffes und damit die Stärke der Bindung zwischen $\mathrm{C}$ und $\mathrm{H}$ erhöht. Diese stärkere Bindung führt bei der Raman-Streuung des Laserlichtes an einem Aromat zu einer Verschiebung der CH-Linie zu größeren Wellenlängen hin. Da Realkraftstoffe Komponenten mit unterschiedlichen Siedetemperaturen enthalten, ließe sich so eine mögliche Fraktionierung von alkanischen und aromatischen Kohlenwasserstoffmolekülen bei der Verdampfung in einem Spray verfolgen.

Bei ersten Versuchen mit gasförmigem Euro Super zeigten sich bräunlich-schwarze Ablagerungen auf den Kammerfenstern an der Stelle wo der Laserstrahl hindurchtrat. Diese waren auf Bitumenrestanteile im handelsüblichen Euro Super-Kraftstoff zurückzuführen, welche das Laserlicht absorbierten und sich auf der Oberfläche der Ein- und Austrittsfenster ablagerten. Zur Vermeidung dieser Probleme wurde in allen folgenden Raman-spektroskopischen Untersuchungen ein von der „Deutschen Shell AG“ zur Verfügung gestellter spezieller Kraftstoff verwendet, der alle Spezifikationen der Euro SuperNorm (DIN EN 228) erfüllt, jedoch keine Bitumenanteile mehr enthält. Dieser farblose Euro Super-Kraftstoff besitzt die für Superkraftstoffe übliche Oktanzahl (ROZ) von 95. Aus der Elementaranalyse (siehe Anhang) lässt sich ein stöchiometrischer Luftbedarf von $14,52 \mathrm{~kg}$ Luft pro Mol bestimmen. Der Durchschnittswert liegt für Superkraftstoffe bei $14,02 \mathrm{~kg} / \mathrm{Mol}$ und für Isooktan bei $15,12 \mathrm{~kg} / \mathrm{Mol}$. 


\subsubsection{Lambda-Wert-Bestimmung in homogenen Gemischen}

Flüssige Kraftstoffe erzeugen auf Grund ihrer sehr viel höheren Dichte ein größeres RamanSignal als gasförmige. Untersuchungen an Raumluft zeigten aber schon, dass das optische Messsystem in der Lage war Sauerstoff und Stickstoff in geringen Konzentrationen zu detektieren. Es sollte daher ebenfalls möglich sein, in Ottomotoren auftretende LuftKraftstoffgemischkonzentrationen zu analysieren. Zunächst sollten tröpfchenfreie und daher für die Messtechnik wesentlich unproblematischere homogene Bedingungen, wie sie in Motoren z.B. bei hohen Drehzahlen vorkommen, untersucht werden.

Die Druckkammer wurde dazu mit verschiedenen Partialdrücken gasförmiger Gemische von Euro Super farblos und Stickstoff homogen aus dem Vormischbehälter befüllt. Der Gesamtdruck betrug dabei 1bar. Für Isooktan lässt sich der Partialdruck von stöchiometrischen Verhältnissen aus der Gleichung 2.2 bestimmen. Er liegt für $\lambda=1$ und einem Gesamtdruck von 1bar bei 16,7mbar und entspricht ungefähr dem von Euro Super. Allerdings bestand das Problem, dass Teile der Druckkammer und ihre Zuleitungen nicht auf eine Temperatur oberhalb von $184^{\circ} \mathrm{C}$ gebracht werden konnten. Bei dieser Temperatur liegt das Siedeende von Euro Super (siehe Anhang, Prüfbericht Euro Super) und somit können bei niedrigeren Temperaturen hochsiedende Komponenten des Kraftstoffes auskondensieren. Der tatsächliche Lambda-Wert des Luft/Kraftstoffgemisches lag daher eher höher.

In den detektierten Raman-Spektren (Abbildung 4.5a) ist zu sehen, dass das StickstoffRaman-Signal bei $608 \mathrm{~nm}$ nahezu konstant blieb, während das Kohlenwasserstoff-Signal mit steigendem Partialdruck zunimmt. Summiert man das CH-Signal zwischen 625nm und 650nm auf (nach Untergrundabzug), so zeigt sich dessen lineare Abhängigkeit vom Partialdruck, wie aus Abbildung 4.5b hervorgeht. Mit diesem optischen Messsystem war es möglich, den Lambda-Wert eines Gemisches mit einer Genauigkeit von etwa 2\% bestimmen zu können. 


\subsubsection{Sprayuntersuchungen}

In Ottomotoren liegen lediglich in einigen Betriebszuständen homogene LuftKraftstoffgemische vor. Im geschichteten Betrieb, d.h. bei einer Kraftstoffeinspritzung in den Brennraum während der Kompression, treten oft tröpfchenbeladene Gasströmungen auf. Um das Verhalten des Detektionssystems in Anwesenheit von Tröpfchen bei einer Anregungswellenlänge von 532nm zu untersuchen, wurde in der Kammer zusätzlich ein BDE-Injektor installiert (siehe Abbildung 4.3). Der Aufnahmezeitpunkt der Kamera war mit dem Laser und der Ansteuerung für das Magnetventil des Injektors synchronisiert, so dass Raman-Messungen zu definierten, frei wählbaren Zeitpunkten während der Einspritzung erfolgten. Zudem konnte durch eine geeignete Wahl der Abbildungslinsen vor dem Spektrographen ein Bereich von 16mm eingesehen werden.

Die ersten Sprayuntersuchungen wurden mit Methanol durchgeführt. Müller et al. [41] hatten eine Methode zur quantitativen Flüssigkeits/Gasphasentrennung mittels RamanSpektroskopie beschrieben. Dieses Verfahren beruht auf der besonderen Eigenschaft der Alkanole im flüssigen Zustand Wasserstoffbrückenbindungen auszubilden, deren energetischer Einfluss sich in der spektralen Position und Bandbreite der Ramanemission der Hydroxylschwingungen niederschlägt.

a.)

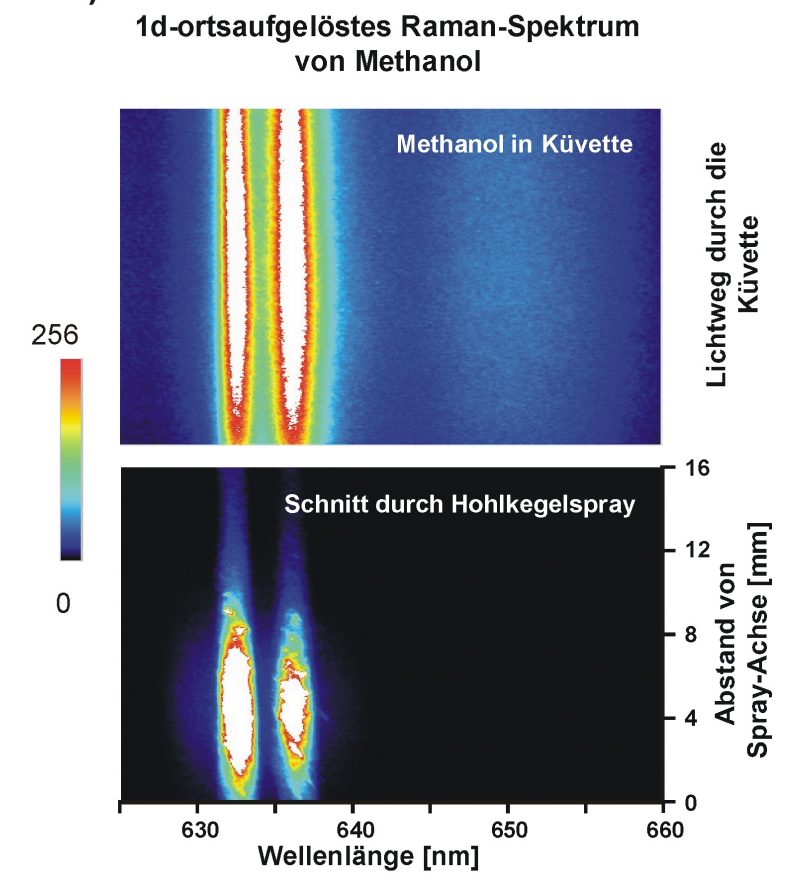

b.)

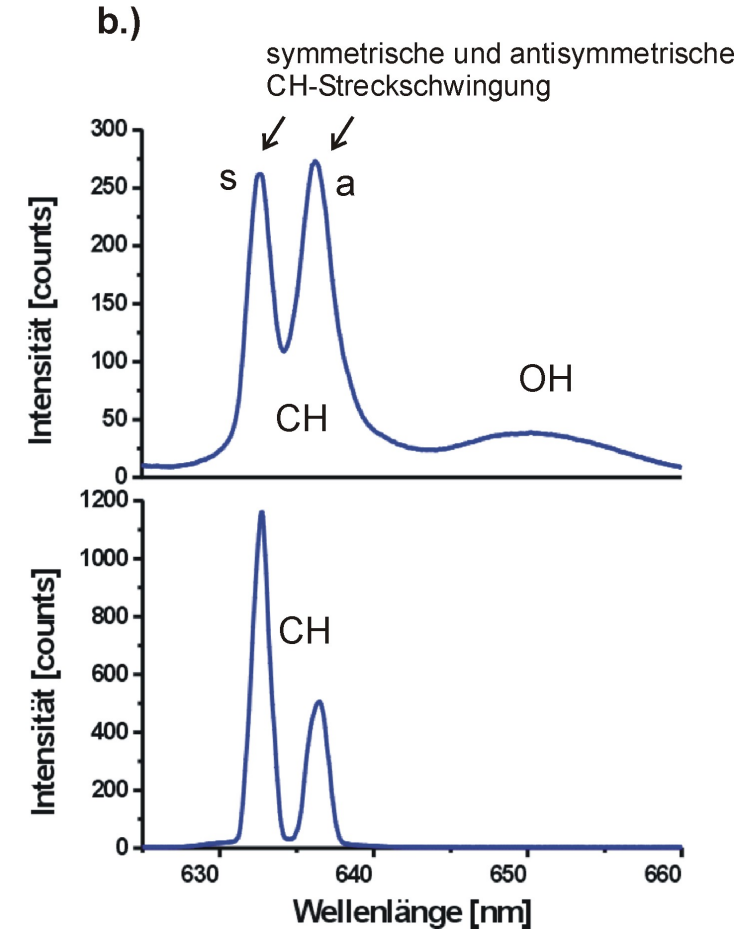

Abbildung 4.6.: Raman-Emission von flüssigem Methanol in einer Küvette und in einem Hohlkegelspray a) ortsaufgelöstes Spektrum b) örtlich gemitteltes Spektralprofil 
a)
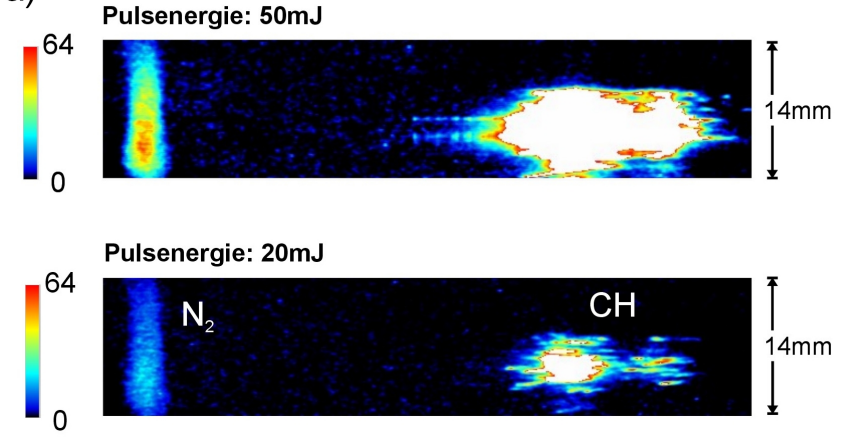

Pulsenergie: $5 \mathrm{~mJ}$

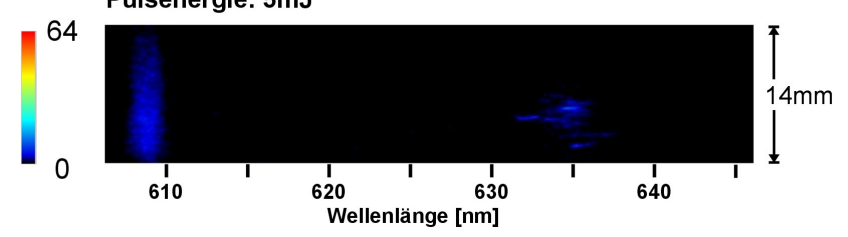

b)

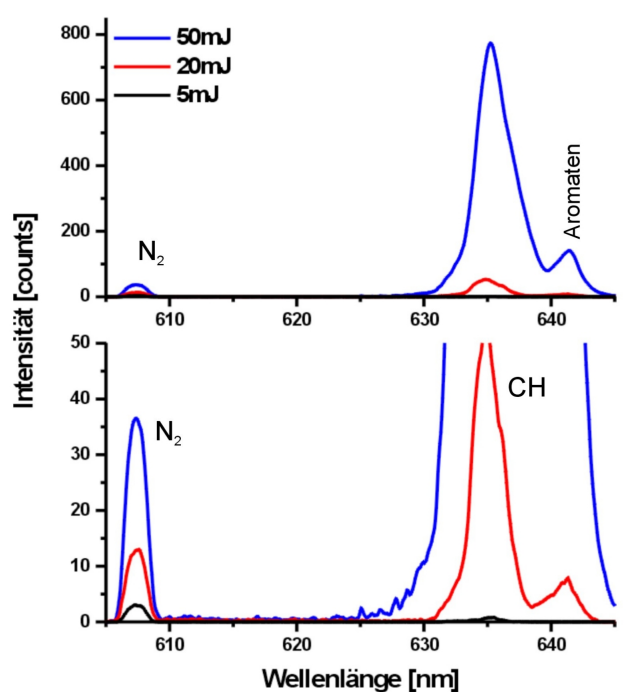

Abbildung 4.7.: Nichtlineares Verhalten der Raman-Emissionen im Euro Super-Spray bei verschiedenen Anregungsenergien a) ortsaufgelöstes Spektrum b) örtlich gemitteltes Spektralprofil

Im Raman-Spektrum von flüssigem Methanol in einer Küvette (Abbildung 4.6 oben) waren zum einen deutlich die beiden Raman-Emissionen der symmetrischen und der antisymmetrischen CH-Streckschwingung zu sehen, die zwischen 630nm und 640nm liegen. Zum anderen trat eine breite Bande der OH-Raman-Emission auf. In der Flüssigphase haben benachbarte Moleküle durch Wasserstoffbrückenbindungen einen Einfluss auf die Stärke der intramolekularen OH-Bindung. Diese Brückenbindung verringert die Anziehung zwischen dem Kohlenstoffatom und der OH-Gruppe beim Methanol und bewirkt so eine energetische Verlagerung der OH-Vibrationsniveaus. Die OH-Schwingung in der Flüssigkeit liegt im Bereich von $\nu_{O H, f l u ̈ s s i g}=3200-3600 \mathrm{~cm}^{-1}$ und damit bei einer Anregungswellenlänge von $532 \mathrm{~nm}$ im Wellenlängenbereich von etwa $641 \mathrm{~nm}$ bis $658 \mathrm{~nm}$. Die Raman-Verschiebung für eine ungestörte OH-Schwingung liegt in der Gasphase bei $\nu_{O H, g a s}=3683 \mathrm{~cm}^{-1}$, würde also bei etwa $662 \mathrm{~nm}$ auftreten.

Ein ähnliches Spektrum sollte sich also auch in einem tröpfchenbeladenen Spray ergeben. In Abbildung 4.6 unten ist das Raman-Spektrum vom Rand eines Spraykegels $10 \mathrm{~mm}$ unterhalb des Injektors kurz nach dem Beginn der Einspritzung dargestellt. Unter diesen Bedingungen bildet das Spray einen kompakten Flüssigkeitskegel und an dieser Stelle gibt es kein gasförmiges Methanol. Im Spektrum sind wieder die beiden $\mathrm{CH}-$ Streckschwingungen zwischen 630nm und 640nm zu sehen. Allerdings war das Verhältnis der Raman-Signal-Intensitäten nicht mehr annähernd gleich groß, sondern hatte sich verschoben. Zudem war das Signal gegenüber der Messung von flüssigem Methanol in der Küvette deutlich intensiver. Die OH-Bande fehlte im Raman-Spektrum jedoch ganz, ob- 
wohl sie in flüssigem Methanol in einem festen Verhältnis zu den CH-Raman-Linien stehen sollte. Aus diesen Untersuchungen wird deutlich, dass im Gegensatz zu den Messungen in der Küvette das Vorhandensein von Tröpfchen einen Einfluss auf die Raman-Emissionen aus der Flüssigphase hat, denn in Sprays von Isooktan und Euro Super war dieser Effekt ebenfalls zu beobachten.

Genauere Untersuchungen zeigten eine starke Abhängigkeit der Kraftstoff-RamanEmission von der eingestrahlten Laserenergie. Dies wird deutlich aus den Raman-Spektren von Euro Super bei der Variation der Laserenergie von $5 \mathrm{~mJ}$ bis $50 \mathrm{~mJ}$, welche in Abbildung 4.7 dargestellt sind. Bei diesen Aufnahmen wurde über 100 Einzelschüsse gemittelt und die Kamera mit 8 Pixeln Binning betrieben. Bei 608nm ist das Stickstoffsignal zu sehen, dessen Intensität linear mit der Anregungsenergie skaliert. Das CH-Raman-Signal des Kraftstoffes dagegen steigt überproportional an. Diese selektive Überhöhung der CH-RamanIntensität erfolgt durch stimulierte Raman-Streuung in den Spraytröpfchen. Transparente Mikrokugeln, wie z.B. Kraftstofftröpfchen in Sprays, können wie winzige Laserresonatoren wirken $[7,16]$. Das bedeutet, dass einfallende Photonen durch vielfache Totalreflektion im Tröpfchen gefangen werden, so dass sich in den Tröpfchen eine ausgesprochen hohe Strahlungsenergiedichte aufbaut. Dies kann bei genügend hoher Molekül- und Photonendichte zur Erzeugung von stimulierter Raman-Streuung in den Tröpfchen führen. Da die stimulierte im Gegensatz zu der spontanen Raman-Streuung ein nichtlinearer Prozess ist, hängt die Signalintensität im wesentlichen stark von der Laserleistung, aber in geringerem Maße auch von der Tröpfchengröße und der Tröpfchenform ab (siehe Abschnitt 4.2.5).

Bei Untersuchungen von Kraftstoffsprays mit ultravioletter Laseranregung [40] wurde keine stimulierte Raman-Streuung in Tröpfchen beobachtet. Dies könnte mit der geringeren Kohärenzlänge der Excimer-Laser von unter $100 \mu \mathrm{m}$ zusammenhängen. In den Tröpfchen mit einem größeren Durchmesser als diese Kohärenzlänge können sich keine Resonanzmoden ausbilden. Dagegen besitzen Nd:YAG-Laser Kohärenzlängen typischerweise von einigen Zentimetern. Zudem ist durch die größere Bandbreite dieser Laser und die damit verbundene Verteilung der Strahlungsenergie auf mehrere Moden die Wahrscheinlichkeit für einen Überlapp mit den Resonanzmoden des Tröpfchens größer.

\subsubsection{Eliminierung nichtlinearer Effekte durch Absorberfarbstoff}

Die beschriebenen nichtlinearen Streuprozesse hatten zur Folge, dass eine Bestimmung des $\lambda$-Wertes in Anwesenheit von Tröpfchen nicht möglich war. Insbesondere das an Stickstoffmolekülen elastisch gestreute Licht der in den Tröpfchen erzeugten überhöhten CHRaman-Streuung lieferte eine Fehlinformation über die tatsächliche örtliche Kraftstoffverteilung. Eine Reduzierung der Laserleistung, um zu verhindern, dass die Lasingschwelle der stimulierten Raman-Streuung in den Tröpfchen überschritten wird, war auf Grund der ohnehin sehr schwachen Raman-Signale durch eine Anregung bei einer Wellenlänge von 532nm nicht möglich. Nur durch ein Verringerung der Resonatorgüte konnte daher 


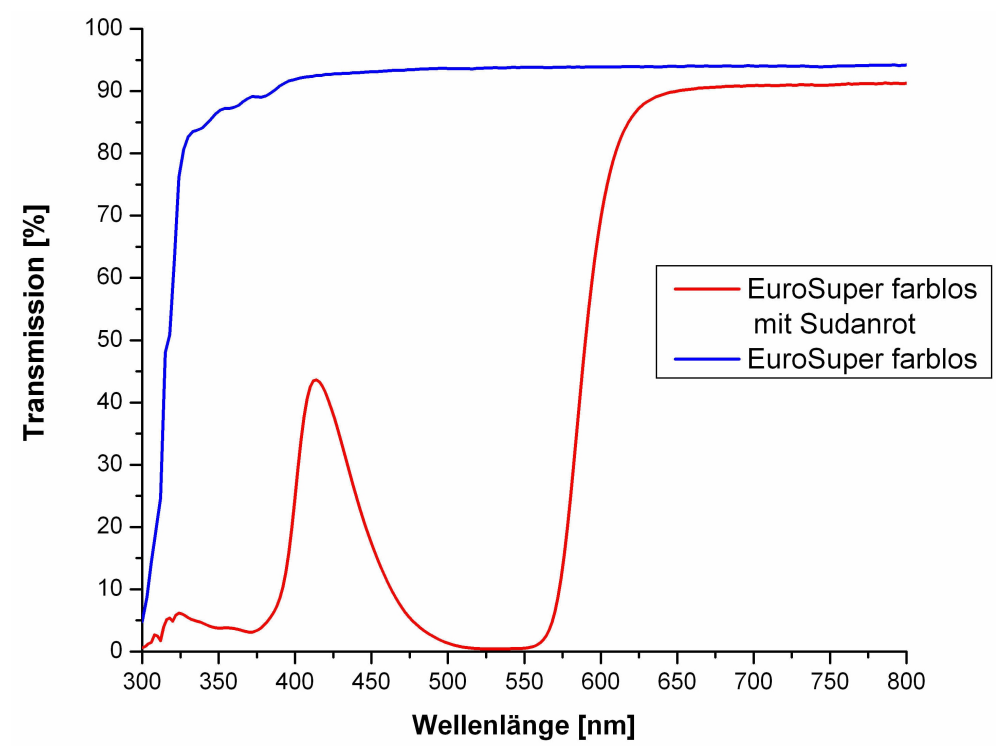

Abbildung 4.8.: Transmission von Euro Super mit und ohne Absorberfarbstoff Sudanrot

eine Erhöhung der Schwelle erzielt werden.

Um dies zu erreichen, wurde dem Kraftstoff Euro Super farblos ein Absorberfarbstoff beigemischt. Es war dabei notwendig, einen Farbstoff zu wählen, der das Laserlicht bei 532nm effektiv absorbiert, aber die von Luft und gasförmigem Kraftstoff erzeugten Raman-Linien zwischen $600 \mathrm{~nm}$ und 650nm transmittiert. Letzteres ist wichtig, da sonst eine Lambda-Wert-Bestimmung im Inneren eines Spraykegels durch die Abschirmung des Raman-Streulichtes durch die Flüssigkeit nicht möglich wäre. Der Azo-Farbstoff Sudanrot erfüllt diese Voraussetzungen (Abbildung 4.9)[18].

Für Sudanrot (auch als Ceresinrot oder engl. Solvent Red 23 bezeichnet) lautet die exakte chemische Nomenklatur: Benzolazo-4-benzolazo-1-(2-naphthol), 1-(4Phenylazophenylazo)-2-naphthol, Aminoazobenzol-azo-b-naphthol mit der Summenformel $\mathrm{C}_{22} \mathrm{H}_{16} \mathrm{~N}_{4} \mathrm{O}$. Unpolare Substanzen wie z.B. Öle, Fette, Harze oder Kohlenwasser-

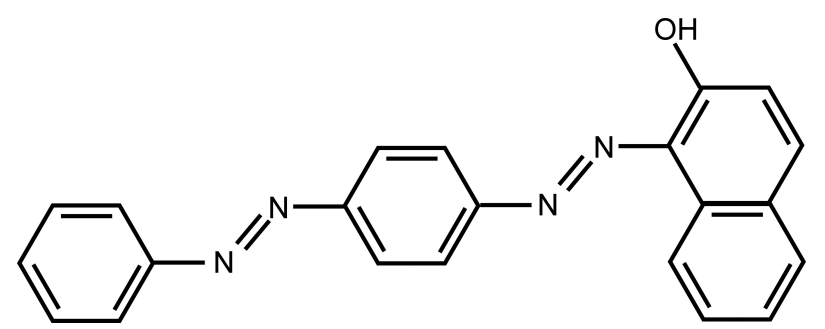

Abbildung 4.9.: Strukturformel von Sudanrot 
a.)

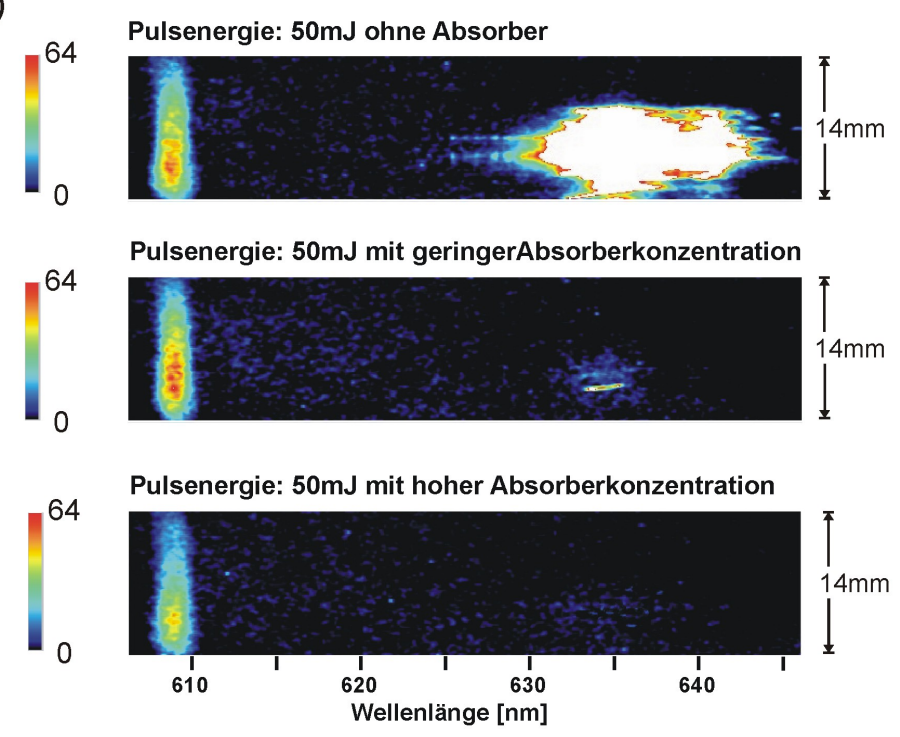

b.)

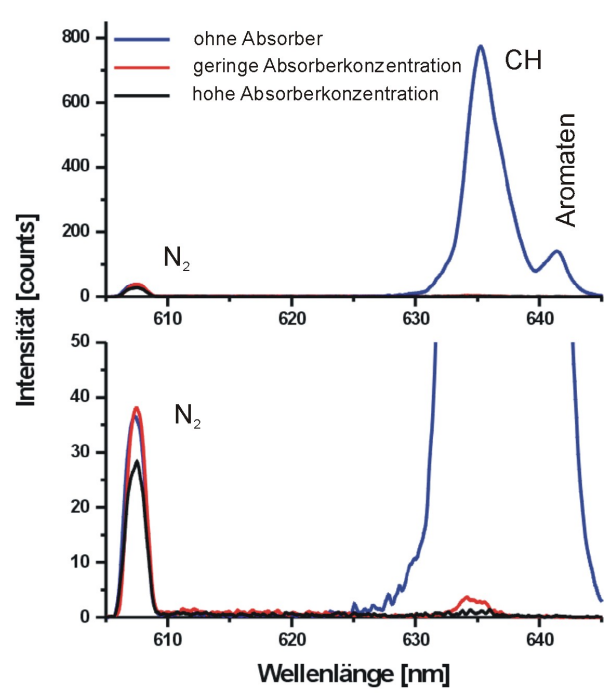

Abbildung 4.10.: Stimulierte Raman-Streuung im Kraftstoffspray in Abhängigkeit von der Konzentration des Absorberfarbstoffes a) ortsaufgelöstes Spektrum b) örtlich gemitteltes Spektralprofil

stoffe können als Lösungsmittel verwendet werden. Aus der Transmissionsmessung von mit Sudanrot versetztem Euro Super farblos $(100 \mu \mathrm{g} / \mathrm{l})$ in einer $1 \mathrm{~mm}$ dicken Flüssigkeitsschicht (Abbildung 4.8) geht hervor, dass die Anregungswellenlänge 532nm vollständig absorbiert wird, die Raman-Signale oberhalb von $600 \mathrm{~nm}$ aber transmittiert werden. Das Raman-Streulicht von Sauerstoff bei 580nm wird durch das Sudanrot allerdings sehr stark abgeschwächt. Daher wurde in allen folgenden Experimenten der Lambda-Wert immer aus dem Verhältnis zwischen dem Stickstoff- und dem Kohlenstoff-Raman-Signal bestimmt unter der Annahme, dass in der Luft im Brennraum des Motors immer ein festes Verhältnis der $\mathrm{N}_{2^{-}}$zur $\mathrm{O}_{2}$-Konzentration herrscht. Diese Bedingung ist solange erfüllt, wie das Gemisch nicht gezündet und verbrannt wird und keine Abgasrückführung in den Brennraum erfolgt. Für die späteren Experimente am Transparentmotor, die in Abschnitt 4.3 beschrieben werden, galt dies. Allerdings ist bei der Verhältnisbildung zu beachten, dass das Stickstoff-Raman-Signal bei 608nm von Sudanrot etwas stärker absorbiert wird als das CH-Raman-Signal bei 635nm. Dieses kommt aber nur bei einer großen Flüssigkeitsmenge zum tragen, z.B. zu sehr frühen Zeitpunkten der Sprayentwicklung.

Sudanrot besitzt einen Schmelzpunkt von $199^{\circ} \mathrm{C}$ und fällt bei der Verdampfung des Kraftstoffes als Festkörper aus. Ablagerungen an den optischen Zugängen der Druckkammer und des Transparentmotors auch nach Laserbeschuss wurden jedoch nicht beobachtet. Bei einer Anregungswellenlänge von 532nm erfolgte eine sehr schwache breitbandige Fluoreszenzemission des Absorberfarbstoffs Sudanrot im Wellenlängenbereich zwischen etwa 400nm und 800nm. Diese Emission konnte bei allen Messungen als Hintergrundsignal vom 
Messsignal abgezogen werden. 
Es wurde nun untersucht, welchen Einfluss der Farbstoff Sudanrot auf die stimulierten Effekte im Kraftstoffspray hat. In Abbildung 4.10 sind die Raman-Spektren aus dem Rand des Sprays bei verschiedenen Konzentrationen des Absorberfarbstoffes dargestellt. Die Bedingungen waren die gleichen, wie bei den Untersuchungen in Abschnitt 4.2.3. Die Laserpulsenergie betrug 50mJ. Schon bei einer geringen Absorberkonzentration in Euro Super farblos von ca. $30 \mu \mathrm{g} / \mathrm{l}$ ist eine deutliche Abnahme der stimulierten CH-RamanEmissionen aus den Tröpfchen zu beobachten, wogegen das Stickstoff-Signal gleich groß bleibt. Erst bei einer hohen Absorberkonzentration von $100 \mu \mathrm{g} / \mathrm{l}$ nimmt das $\mathrm{N}_{2}$-RamanSignal ab. Das CH-Raman-Signal verschwand dabei nahezu vollständig.

Durch den Absorberfarbstoff wird somit das Laserlicht absorbiert und so die Resonatorgüte der Tröpfchen soweit gesenkt, dass keine stimulierten Effekte auftraten. Eine Bestimmung des Luft/Kraftstoffverhältnisses im Brennraum eines Ottomotors ist mit dieser Methode damit erstmalig auch in tröpfchenbeladenen Strömungen und unter Verwendung von Realkraftstoff möglich.

\subsubsection{Resonatoreigenschaften von Flüssigkeitströpfchen}

Die Flüssigkeit eines Sprays verlässt die Düse zunächst als kompakter Strahl. Dieser bildet dann wellenförmige Strukturen, anschließend Ligamente und zerfällt dann in einzelne Tröpfchen. Dabei treten nicht nur sphärische, sondern auch oblate und prolate Tröpfchenformen auf, die auf Grund des Entstehungsprozesses vibrieren. Genauere Untersuchungen an einzelnen Kraftstofftröpfchen sollten nun Aufschluss über die Entstehung von stimulierter Raman-Streuung in Tröpfchen bei Laseranregung und den Einfluss des Absorberfarbstoffes darauf geben. Insbesondere sollte geklärt werden, welche Absorberkonzentration für eine hohe Lasingschwelle notwendig ist.

Wird ein Flüssigkeitströpfchen mit kohärentem Licht bestrahlt, so können verschiedene Effekte durch die sphärische Morphologie auftreten. Erstens wird in das Tröpfchen eingekoppeltes Laserlicht auf der laserabgewandten Seite fokussiert und führt dort zu hohen Feldstärken. Dieser Effekt ist in Abbildung 4.11 skizziert. Durch das senkrecht einfallende Laserlicht entsteht ein weiterer Bereich hoher Feldstärke auf der laserzugewandten Seite des Tröpfchens. Mit Hilfe der Lorenz-Mie-Theorie lassen sich diese Effekte beschreiben. Dabei ergibt sich, dass die Feldstärke im Fokuspunkt der laserabgewandten Seite etwa 100mal größer ist, als in der gegenüberliegenden Seite $[39,55]$. Die Energiedichte in diesen beiden Bereichen nimmt mit steigendem Tröpfchenradius $a \mathrm{zu}$.

Zweitens kann sich das Tröpfchen wie ein optischer Resonator verhalten. Da der Brechungsindex eines Kraftstofftröpfchens größer ist als der der umgebenden Luft, gibt es einen Brechungsindexsprung und damit die Möglichkeit der Totalreflexion von im Tropfen befindlichem Licht an der Tröpfchenoberfläche. Experimentell ergab sich bei Untersuchungen von Beugungserscheinungen mit einem Helium-Neon-Laserstrahl für alle hier verwendeten Kraftstoffe (Methanol, Isooktan und Euro Super) ein Brechungsindex von 1,36. 


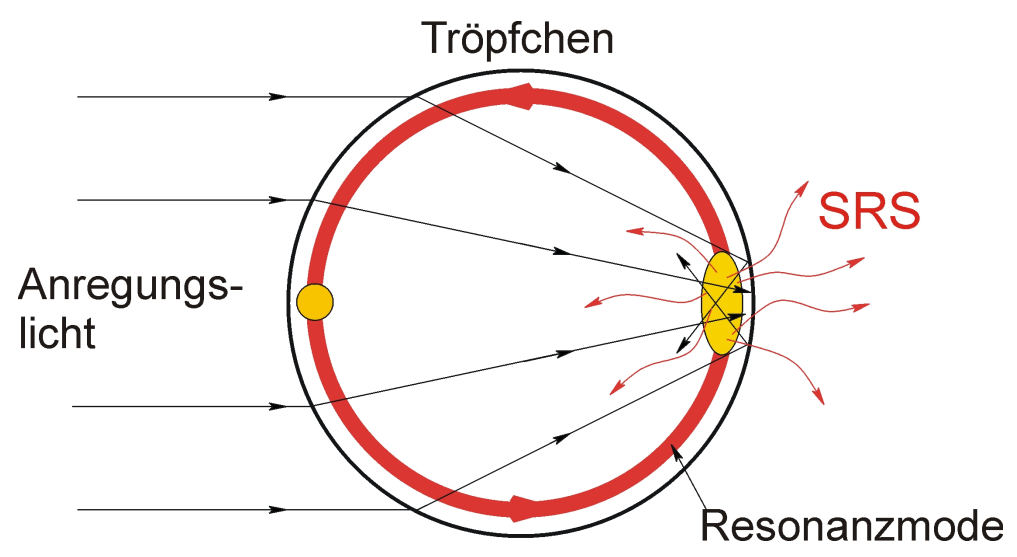

Abbildung 4.11.: Entstehung von stimulierter Raman-Streuung durch Resonanzmoden in Tröpfchen

Wird Anregungslicht in den Tropfen eingekoppelt oder im Tropfeninneren Licht erzeugt, beispielsweise durch spontane Raman-Streuung, so kann dies an der Grenzschicht Tröpfchen/Luft beim Auftreffen unter Winkeln größer als der Grenzwinkel der Totalreflexion vollständig reflektiert werden und weiter umlaufen. Trifft das Licht nach einem Umlauf im Tröpfchen phasengleich wieder auf seinen Ausgangspunkt, so führt dies zu einer konstruktiven Interferenz und der Ausbildung von morphologieabhängigen Resonanzmoden (morphology dependent resonance - MDR) [17, 8]. Diese Resonanzen entstehen, wenn die Länge des Lichtlaufweges im Tröpfchen einem ganzzahligen Vielfachen der Wellenlänge entspricht. Die Schichtdicke $d$, in denen die Resonanzmoden auftreten, hängt mit dem Grenzwinkel der Totalreflexion zusammen und ergibt sich aus den Brechungsindizes der Flüssigkeit $n_{f}$ und der umgebenden Luft $n_{l}$ für ein Tröpfchen mit Radius $a$ :

$$
d=a\left(1-\frac{n_{l}}{n_{f}}\right)
$$

Sowohl für eingekoppeltes Laserlicht als auch für im Tröpfchen entstandenes RamanStreulicht können Resonanzmoden existieren. Sind die Verluste eines Umlaufes geringer als die Verstärkung durch den Resonator, können nichtlineare Effekte auftreten. Überlagert sich nicht nur das in den zwei Bereichen fokussierte Anregungslicht mit der Resonanzmode des Raman-Streulichtes sondern existiert auch eine zu dieser passende Resonanzmode des Anregungslichtes, so tritt starke stimulierte Raman-Streuung auf. Der Gütefaktor $Q$ des optischen Resonators im Tröpfchen ist definiert als das Verhältnis der im Resonator befindlichen Energie zu den Verlusten pro Umlauf:

$$
Q=\frac{\text { Energie im Resonator }}{\text { Energieverlust pro Umlauf }}
$$

Die Schwelle, ab der Lasing oder stimulierte Raman-Streuung auftritt, sinkt mit steigender Tröpfchengröße. Die Ursache hierfür ist zum einen, dass mehr Licht in ein größeres Tröpfchen durch den größeren Querschnitt eingekoppelt wird, zum anderen vergrößert 
sich die Anzahl der Moden und damit die Eindringtiefe der Mode ins Tröpfcheninnere. Letzteres verbessert den räumlichen Überlapp der Resonanzmode mit den zwei Gebieten hoher Intensität [9]. Die Lasingschwelle liegt für in einem Kraftstoffspray auftretende Tröpfchengrößen bei einer Resonatorgüte von $Q=10^{6}$ bis $10^{8}$ [1]. Exakte theoretische Vorhersagen über die Lasingschwelle gestalten sich jedoch sehr schwierig.

Als Drittes kann die Fluoreszenz und der Raman-Streuquerschnitt für spontane Emission der Moleküle im Tropfen größer sein als die in einem kompakten Flüssigkeitsvolumen. Für in einer Flüssigkeit gestreute Photonen sind die Wellenfunktionen im Kontinuum die erlaubten Endzustände. In einem Tropfen werden allerdings die morphologieabhängigen Resonanzmoden bevorzugt und die Emission bei anderen Wellenlängen gehemmt [36, 10].

Für die experimentellen Untersuchungen der Raman-Streuung in Tröpfchen wurden diese mit definiertem Durchmesser mittels eines Tröpfchengenerators [37] erzeugt. Dabei gelangt der Kraftstoff über eine Zuleitung unter leichtem Überdruck von 0,3bar $0,8 \mathrm{bar}$ in ein metallenes Rohrstück. Dieses ist mit einer kleinen Blende (Durchmesser $100 \mu \mathrm{m})$ abgeschlossen, durch die die Flüssigkeit herausgedrückt wird. Das Rohrstück ist von einem ringförmigen Piezo-Element umgeben, welches über einen Frequenzgenerator zu Schwingungen im Bereich von 20-120kHz angeregt wird. Die dadurch dem austretenden Strahl aufgeprägten Schwingungen führen zu einer periodischen Strahleinschnürung und letztendlich zu einer gleichmäßigen Tröpfchenbildung. Der Durchmesser der entstehenden Tröpfchen entspricht immer ungefähr dem doppelten Blendendurchmesser.

Das Strahlprofil des Nd:YAG-Lasers wurde über ein Zylinderlinsenteleskop verkleinert (Strahlquerschnitt: $1 \mathrm{~mm} \times 4 \mathrm{~mm}$ ) und der Tröpfchenstrahl so direkt vom Laserlicht beleuchtet. Die Pulsenergie betrug 50mJ. Die Abbildung der beobachteten Tropfen erfolgte mit Hilfe eines Fernfeldmikroskops (Questar - QM100) auf die bildverstärkte CCDKamera. Um das Raman-Streulicht aus dem Gesamtstreulicht des Tröpfchens herauszufiltern wurde ein Bandpassfilter (siehe Abschnitt 4.5.2) in den Strahlengang vor der CCD-Kamera plaziert. Alle diese Untersuchungen erfolgten mit Euro Super als Kraftstoff. In Abbildung 4.12 sind beispielhaft einige Aufnahmen von Tröpfchen dargestellt in denen stimulierte Raman-Streuung auftrat. Bei geringer Pulsenergie sind zunächst nur die heller leuchtenden Bereiche auf der Laser zu- und abgewandten Seite der reinen Kraftstofftröpfchen zu beobachten. Wurde die Pulsenergie erhöht, so zeigten sich an verschiedenen Positionen an der Tröpfchenoberfläche helle Bereiche. Ab einer Pulsenergie von etwa 5mJ kam es vereinzelt zu stimulierter Raman-Streuung, die sich in blitzartigen Überstrahlungen bemerkbar machte.

Nun wurden dem Kraftstoff Euro Super verschiedene Konzentrationen des in Abschnitt 4.2.4 vorgestellten Absorberfarbstoffes Sudanrot beigemischt. Dabei zeigte sich, dass mit steigender Konzentration immer höhere Laserenergien notwendig waren, um die Schwelle für stimulierte Raman-Streuung zu erreichen (Tabelle 4.1). Bei Raumtemperatur löste sich in 1 Liter Euro Super maximal nur etwa $100 \mu \mathrm{g}$ Sudanrotpuler rückstandsfrei. Da bei dieser Konzentration auch die Lasingschwelle am höchsten war, wurde diese bei allen weiteren Untersuchungen verwendet. Bereits im vorherigen Abschnitt wurde in Spray- 


\begin{tabular}{|c|c|}
\hline Absorberkonzentration & Lasingschwelle \\
\hline ohne & $5 \mathrm{~mJ}$ \\
$20 \mu \mathrm{g} / 1$ & $7 \mathrm{~mJ}$ \\
$40 \mu \mathrm{g} / 1$ & $12 \mathrm{~mJ}$ \\
$60 \mu \mathrm{g} / 1$ & $18 \mathrm{~mJ}$ \\
$80 \mu \mathrm{g} / 1$ & $22 \mathrm{~mJ}$ \\
$100 \mu \mathrm{g} / 1$ & $25 \mathrm{~mJ}$ \\
\hline
\end{tabular}

Tabelle 4.1.: Schwelle für stimulierte Raman-Streuung bei verschiedenen Absorberkonzentrationen

untersuchungen gezeigt, dass bei dieser Konzentration die nichtlinearen Effekte deutlich abnehmen. In den in Abbildung 4.12 gezeigten Aufnahmen von Tröpfchen ist deutlich zu sehen, dass die Emissionen aus den absorberversetzten Tröpfchen um einen Faktor 1000 bis 2000 geringer sind, als bei den Tröpfchen mit reinem Euro Super farblos. Dies ist anhand der unterschiedlichen Intensitätsskalen erkennbar.

Mit Hilfe des Absorberfarbstoffes ist es also möglich, die Resonatorgüte $Q$ der Tröpfchen zu senken und damit die Schwelle für stimulierte Raman-Streuung zu erhöhen. 


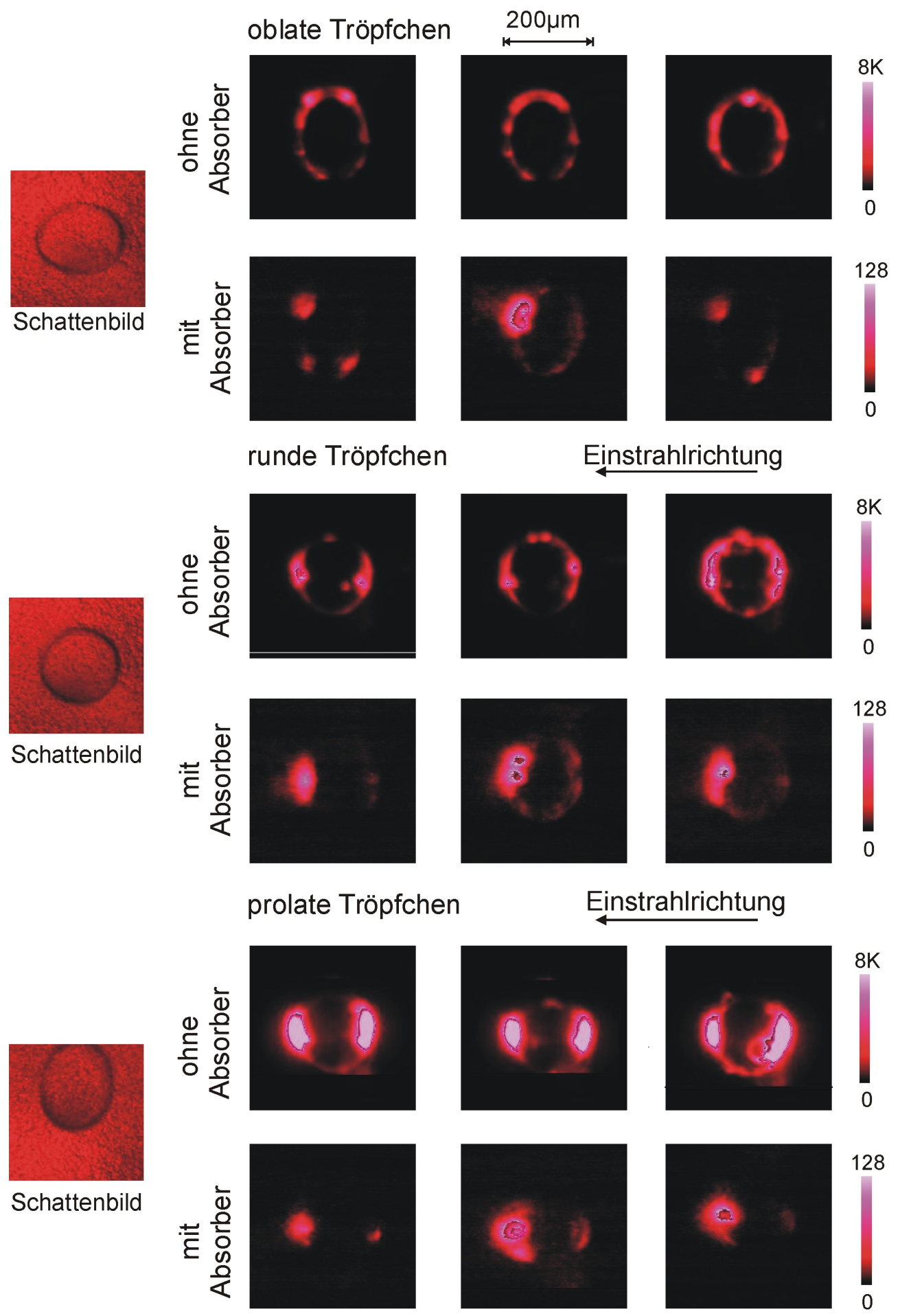

Abbildung 4.12.: Raman-Emission aus Einzeltröpfchen mit und ohne Absorberfarbstoff (gleiche Laserenergie und Aufnahmeparameter, aber verschiedene Intensitätsskalierung) 


\subsection{Motorische Untersuchungen}

Die Einsatzfähigkeit des vorgestellten Raman-spektroskopischen Detektionsverfahrens unter Realkraftstoffbedingungen und tröpfchenbeladenen Strömungen im Brennraum konnte an einem Transparentmotor unter Beweis gestellt werden. In einer mehrwöchigen Messkampagne im Forschungszentrum eines Auftraggebers aus der Automobilindustrie wurden Untersuchungen durchgeführt, bei denen verschiedene Einspritzdüsen für ein strahlgeführtes Brennverfahren (siehe Kapitel 2.1) charakterisiert wurden. Mit Hilfe des 1-dimensional ortsaufgelösten Messverfahrens sollte dabei der optimale Abstand der Zündkerze von der Einpritzdüse gefunden werden, bei dem der $\lambda$-Wert des Luft/Kraftstoffgemisches in den meisten Zyklen zum Zündzeitpunkt nahe dem Wert 1 liegt. Drei Injektortypen (Drallventil, Mehrlochventil und Ringspaltventil) wurden untersucht und Parameter wie Einspritzzeitpunkt, Raildruck und Einbaulage variiert. Daneben zeigten auch durch die Einlassventile erzeugte Wirbelströmungen im Zylinder, Drosselklappen im Ansaugrohr und Mehrfachpulse der Einspritzdüsen einen Einfluss auf die Gemischbildungsprozesse.

Im folgenden Abschnitt werden die Durchführung und Ergebnisse dieser Untersuchungen insbesondere im Hinblick auf die Einsatzfähigkeit des Messsystems dargestellt. Jeweils nach dem Ansteuerende (ASE) der Einspritzdüse wurden 7 Aufnahmezeitpunkte der Kamera im Abstand von $3^{\circ} \mathrm{KW}$ gewählt. Zusätzlich zu der 1-dimensional ortsaufgelösten Bestimmung des $\lambda$-Wertes mit Hilfe der spontanen Raman-Streuung wurde parallel dazu eine 2-dimensionale LIF-Visualisierung der Kraftstoffverteilung mittels eines UV-Excimerlasers durchgeführt. Neben der Analyse der Kraftstoffverteilung am Ort der Raman-Messungen im Brennraum sollte so die Möglichkeit untersucht werden, ob aus der gemessenen Fluoreszenzintensität eine flächige Lambda-Wert-Verteilung ermittelbar ist. Denn als Folge der Fluoreszenzlöschung durch die Stöße zwischen Molekülen ist der $\lambda$-Wert bei hohen Drücken umgekehrt proportional zur Fluoreszenzintensität (siehe Abschnitt 3.2). Eine Kallibration wäre dabei über das aus dem Raman-Signal bestimmten Luft/Kraftstoffverhältnis möglich.

\subsubsection{Experimenteller Aufbau am Transparentmotor}

Der verwendete Versuchsmotor war ein 1-Zylinder Glasringmotor mit einem Bohrungsdurchmesser von $80 \mathrm{~mm}$, der mittels eines Elektromotors geschleppt betrieben wurde. Da nur die Gemischbildung analysiert werden sollte, war eine Zündung des Gemisches nicht vorgesehen. Der obere Teil der Zylinderwand bestand aus einem Quarzglasring, so dass der Brennraum von allen Seiten einsehbar war. Desweiteren war der Kolbenboden ebenfalls mit einem Quarzglasfenster versehen und ermöglichte so über Langlöcher in einem verlängerten Kolben und einen im Kolben plazierten Aluminiumspiegel den Einblick in den Brennraumgiebel. Der schematische Aufbau ist in Abbildung 4.13, 4.14 und 4.15 skizziert. 


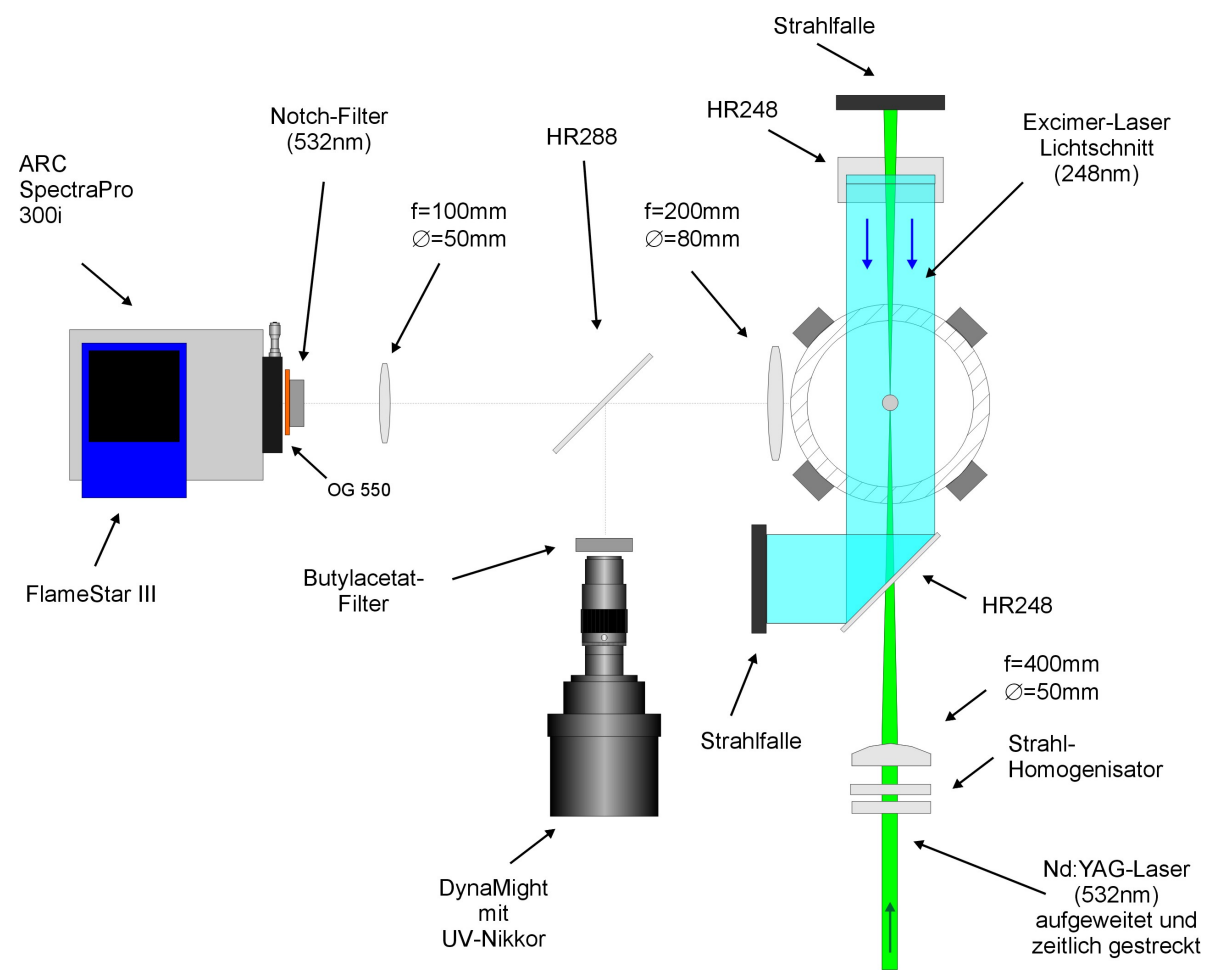

Abbildung 4.13.: Experimenteller Aufbau am Transparentmotor - Aufsicht

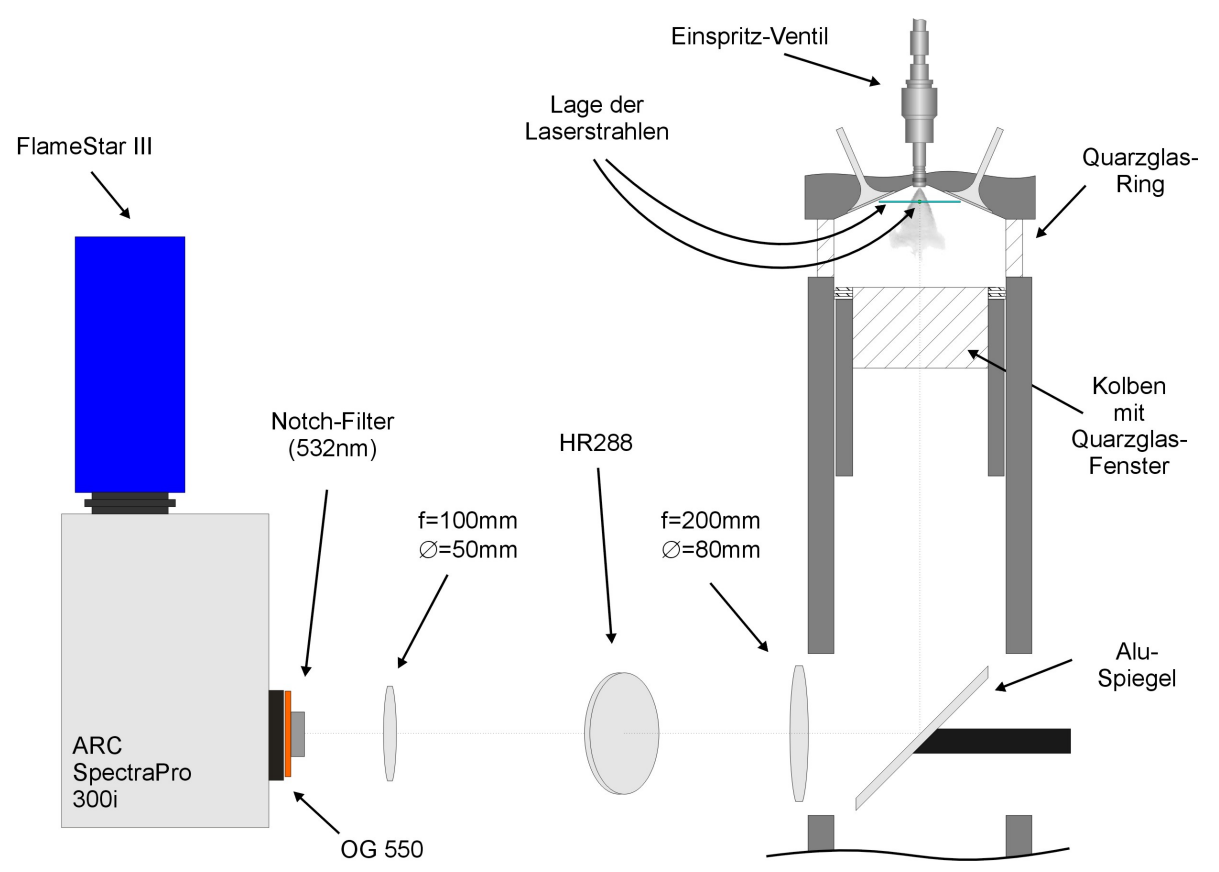

Abbildung 4.14.: Experimenteller Aufbau am Transparentmotor - Seitenansicht frontal 


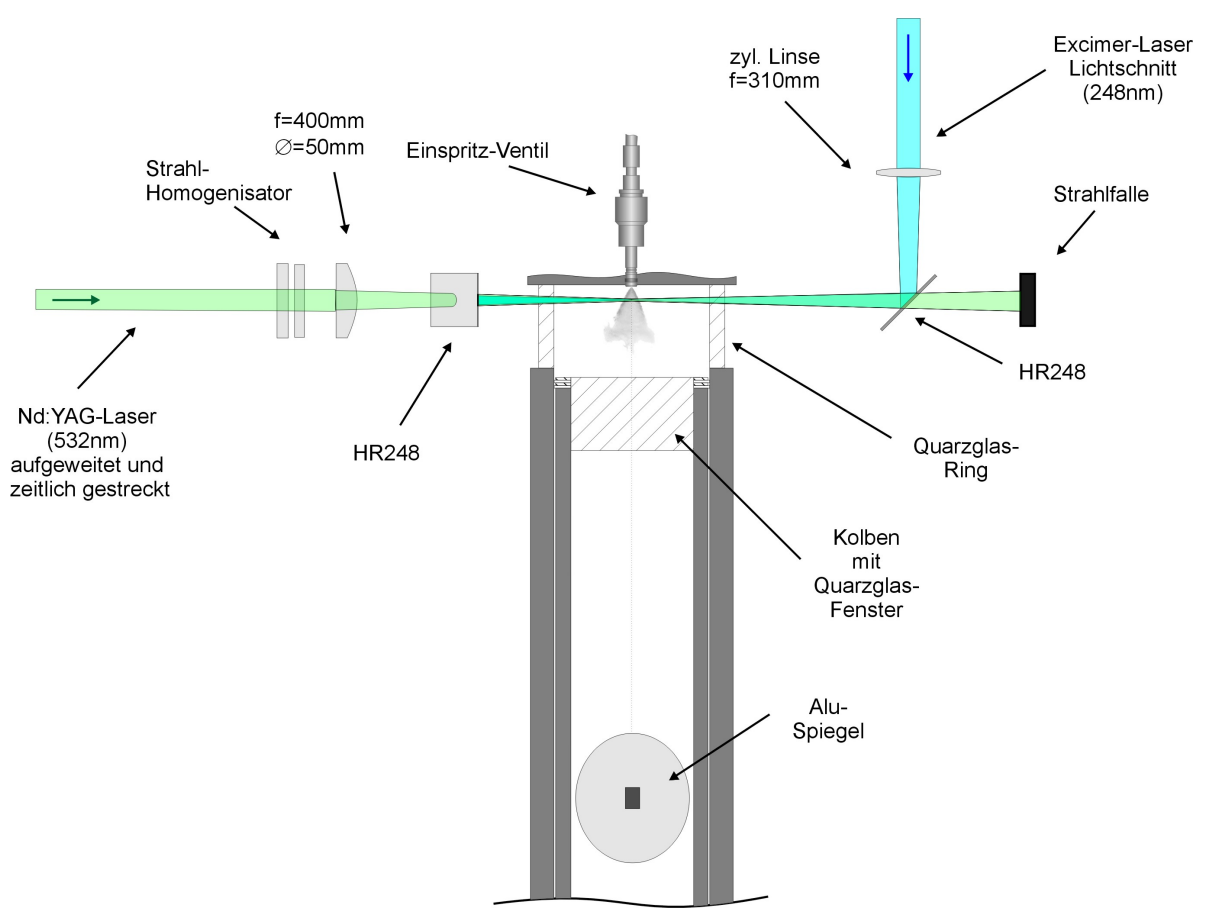

Abbildung 4.15.: Experimenteller Aufbau am Transparentmotor - Seitenansicht

Die Laseranregung bei 532nm und die Detektion der spontanen Raman-Emission erfolgte, wie in den Vorversuchen (Abschnitt 4.2) beschrieben, 10mm unterhalb des Einspritzventils. Die Polarisation des Nd:YAG-Laserstrahls war so angepasst, dass die Dipolabstrahlung der induzierten Raman-Streuung vertikal war und so durch den Kolbenboden ausgekoppelt und über den Aluminiumspiegel auf den Spektrographen abgebildet werden konnte.

Zusätzlich wurde das als flaches Lichtband geformte UV-Licht eines KrF-ExcimerLasers (Lambda-Physik - LX150) bei 248nm in Höhe des grünen Laserstrahls horizontal durch den Glasring gestrahlt. Das vom KrF-Laserstrahl angeregte Fluoreszenzlicht wurde ebenfalls durch den Kolbenboden beobachtet und mittels eines bei 288nm hochreflektierenden Spiegels aus dem Strahlengang ausgekoppelt und auf eine bildverstärkte UV-CCD-Kamera mit 16bit-Dynamik (LaVision - DynaMight) abgebildet. Ein vor der Kamera plazierter Kantenfilter (Butylacetat-Flüssigfilter mit einer Schichtdicke von 1cm) absorbierte das Rayleigh- und Mie-gestreute UV-Anregungslicht bei $248 \mathrm{~nm}$ vollständig.

Die Aufnahmefrequenz des Detektionssystems war durch die festgelegte Wiederholrate von etwa $10 \mathrm{~Hz}$ des Nd:YAG-Lasers begrenzt. Mit Hilfe einer speziell für die Synchronisation des Motors mit dem Laser entwickelten Elektronik (LaVision - YEX-Modul) wurde die Wiederholrate des Lasers so zwischen $9 \mathrm{~Hz}$ und $11 \mathrm{~Hz}$ verschoben, dass abhängig von der Drehzahl des Motors möglichst häufig der gewünschte Beobachtungszeitpunkt und der Laserpuls zusammenfielen und damit wenig Motorzyklen auftraten, in denen keine 
Aufnahmen erfolgten. Die Ansteuerung für den Excimer-Laser und die UV-Kamera war gegenüber dem Nd:YAG-Laser um einige 100ns zeitversetzt, um eine gegenseitige Beeinflussung der beiden Messungen zu vermeiden. Dies ist erlaubt, da die Gemischbewegung innerhalb dieser kurzen Zeit nur sehr klein ist.

\subsubsection{Ergebnisse der Freistrahldetektion}

Die mit Hilfe des UV-angeregten Fluoreszenzlichtes des Kraftstoffs sichtbar gemachten Sprayentwicklungen der drei untersuchten Injektortypen sind in Abbildung 4.16 dargestellt. Um einen Eindruck von der räumlichen Sprayausbreitung zu erhalten, sind die Aufbauskizzen und Schattenaufnahmen des Spraykegels (mit 10mm Größenmaßstab) ebenfalls gezeigt. Das Ansteuerende (ASE) des Einspritzventils, also der Zeitpunkt an dem die Einspritzung des Kraftstoffes beendet wird, lag in allen drei gezeigten Fällen bei $30^{\circ} \mathrm{KWvOT}$ bei einem Raildruck von 100bar. Die 2-dimensionalen Aufnahmen sind jeweils über 100 Einzelschüsse gemittelt. Der Laserstrahl wird beim Durchtritt durch flüssige Anteile des Kraftstoffs abgeschwächt. Dadurch entstehen Intensitätsunterschiede von der rechten zur linken Seite der Bilder. Das Einspritzventil befindet sich bei allen 2-dimensionalen LIFBildern in der Mitte des Bildes.

Im Inneren des Drallventils wird in der Drallkammer der flüssige Kraftstoff in Rotation versetzt. Nach dem Öffnen des Ventils schießt zunächst ein sehr schlanker Flüssigkeitsstrahl aus der Düsenöffnung, der so genannte Vorstrahl. Dieser ist in den Fluoreszenzaufnahmen bis etwa $18^{\circ} \mathrm{KWvOT}$ zu sehen. Erst etwa $400 \mu$ s nach Einspritzbeginn baut sich durch die rotierende Flüssigkeit ein Hohlkegel von der Spitze des Injektors her auf und formt so den Hauptstrahl. Durch den sich im Spraykegel aufbauenden Unterdruck, wird Luft ins Innere des Sprays gezogen (air-entrainment) und damit die Verdampfung des Kraftstoffes beschleunigt. Eine detailliertere Darstellung dieses Vorgangs hat Müller [40] gezeigt.

Das Mehrlochventil erzeugt zehn sehr kompakte Einzelstrahlen, welche so ringförmig angeordnet sind, dass sie einen Spalt freilassen (hier an der rechten Seite). An dieser Stelle wird normalerweise die Zündkerze positioniert, um sie nicht direkt mit Kraftstoff zu benetzen. Die Einzelstrahlen sind ebenfalls bis etwa $18^{\circ} \mathrm{KWvOT}$ sichtbar, bevor sie sich zu einem Vollkegel verbinden.

Im Gegensatz zum Drallventil besitzt das Ringspaltventil keinen Vorstrahl und erzeugt einen sehr viel dünneren und ausladenden Hohlkegel, der dadurch schneller verdampft. Bei diesem Ventil wird eine Wirbelströmung erzeugt, die den Kraftstoff wieder nach oben in Richtung Brennraumgiebel trägt, was in den Fluoreszenzaufnahmen ab etwa $18^{\circ} \mathrm{KWvOT}$ als äußerer Ring erkennbar ist. 

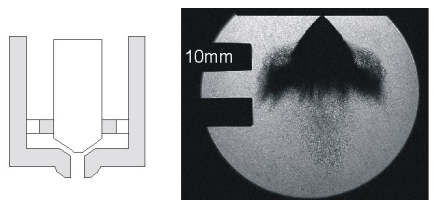

Drallventil

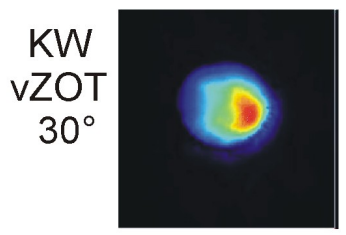

$27^{\circ}$

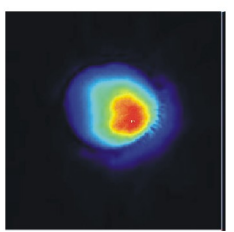

$24^{\circ}$

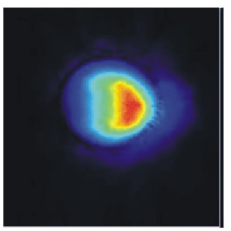

$21^{\circ}$

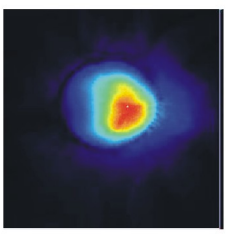

$18^{\circ}$

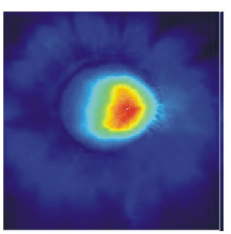

$15^{\circ}$

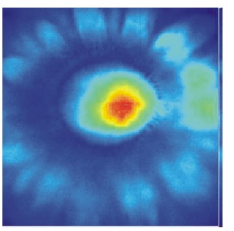

$12^{\circ}$

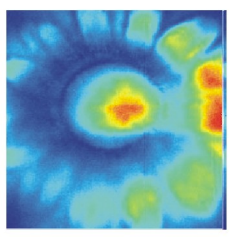

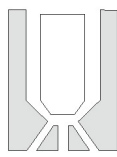

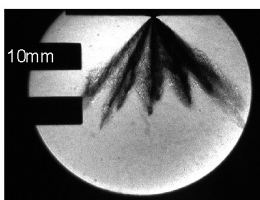

Mehrlochventil

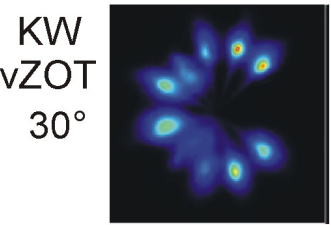

$27^{\circ}$

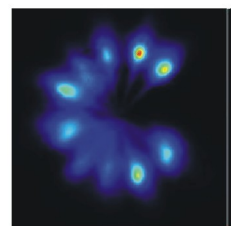

$24^{\circ}$

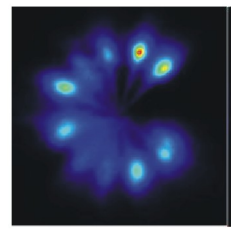

$21^{\circ}$

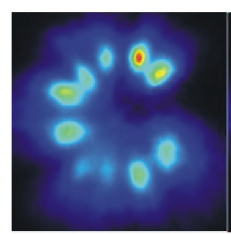

$18^{\circ}$

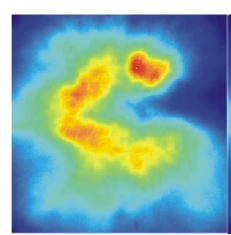

$15^{\circ}$

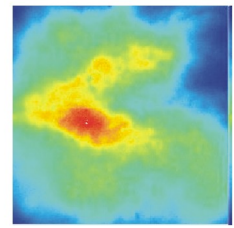

$12^{\circ}$

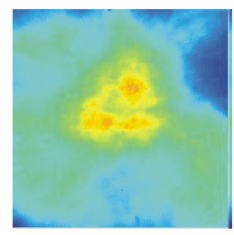

$\max$

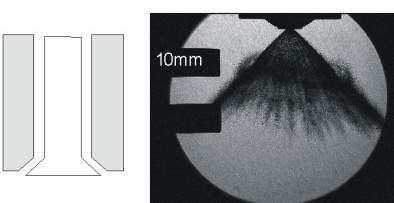

Ringspaltventil

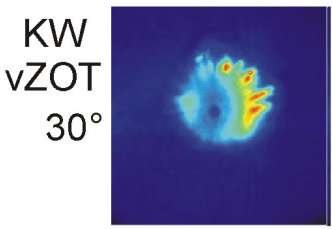

$27^{\circ}$

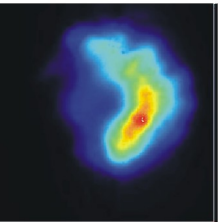

$24^{\circ}$

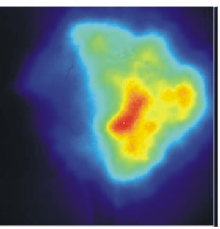

$21^{\circ}$

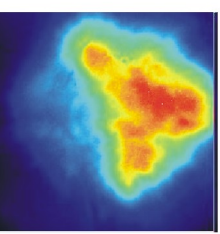

$18^{\circ}$

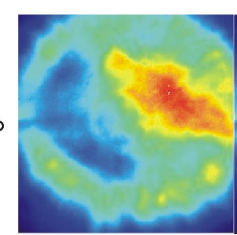

$15^{\circ}$

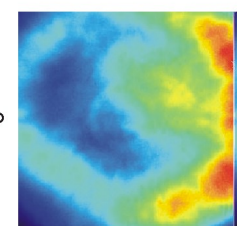

$12^{\circ}$

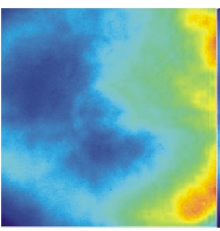

0

Einstrahlrichtung

Abbildung 4.16.: UV-LIF-Aufnahmen der Sprayentwicklung der drei untersuchten Injektoren $\left(\mathrm{ASE}=30^{\circ} \mathrm{KWvOT}\right.$, Raildruck $\left.=100 \mathrm{bar}\right)$ 
a.)
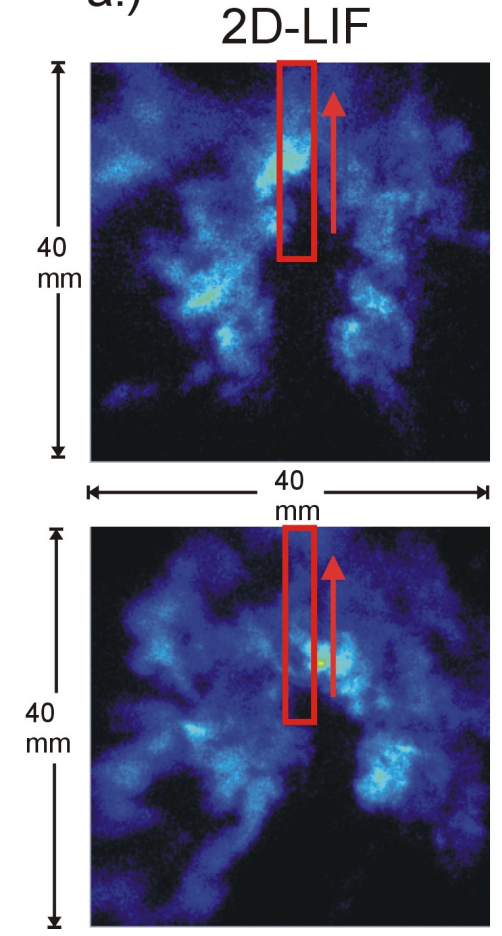

b.)
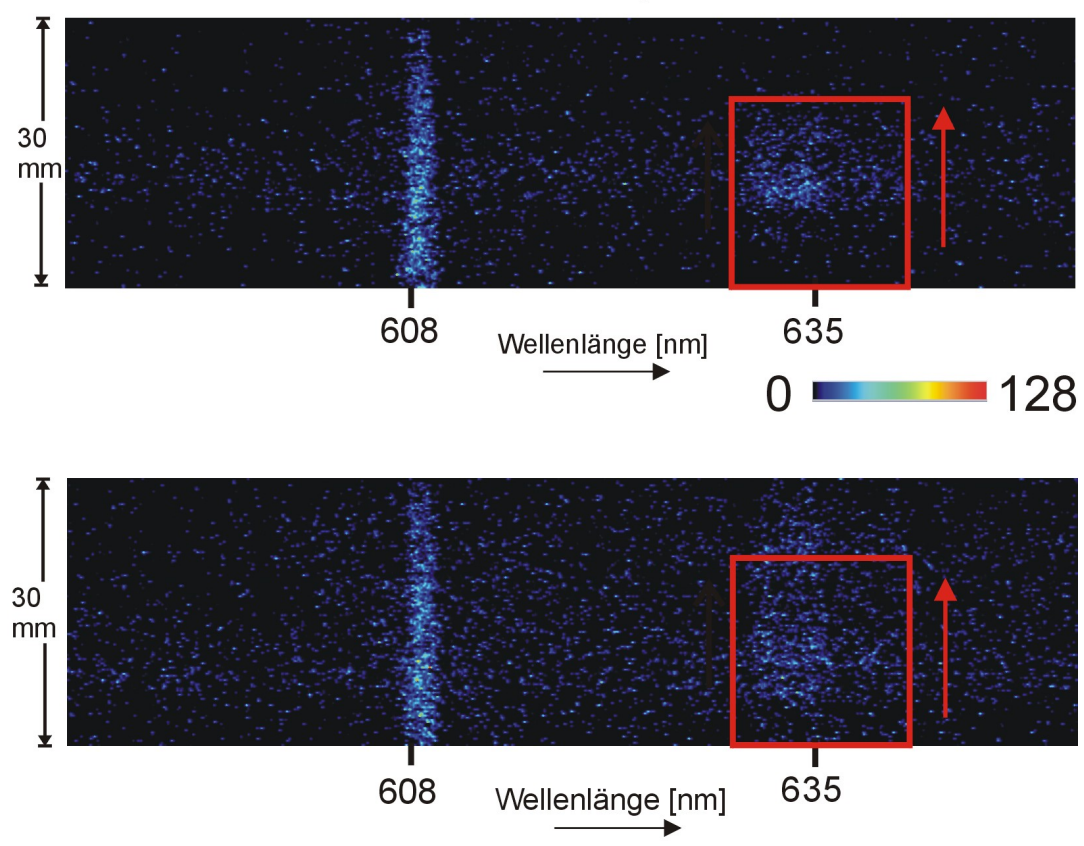

Abbildung 4.17.: Korrelation zwischen a) LIF-Einzelaufnahmen und b) den dazugehörigen RamanSpektren am Beispiel zweier Einspritzungen des Mehrlochventils. Der rote Pfeil gibt die Orientierung des gemeinsamen, umrandeten Messvolumens an

In Abbildung 4.17 sind zwei LIF-Einzelaufnahmen von Einspritzungen des Mehrlochventils mit den dazugehörigen Raman-Spektren beispielhaft gezeigt, um die Korrelation zwischen den beiden Techniken zu verdeutlichen. Die örtliche Auflösung der RamanSignale betrug 0,25mm bei der gewählten Abbildung und dem Pixel-Binning der CCDKamera (siehe Abschn. 4.1.2). Im Spektrum ist das Raman-Signal von Stickstoff bei einer Wellenlänge von $608 \mathrm{~nm}$ und das vom Kraftstoff bei $635 \mathrm{~nm}$ erkennbar. Im oberen Beispiel ist in der Mitte des eingerahmten Messvolumens eine starke UV-Laser-induzierte Fluoreszenz des Kraftstoffes zu sehen, im unteren Teil, also am Injektor, ist diese nicht sichtbar. Das selbe zeigt sich im dazu gehörigen Raman-Spektrum. Dabei gibt der rote Pfeil die Orientierung des Messvolumens an. Im unteren Beispiel sieht man in der LIF-Aufnahme gleichmäßig verteilten Kraftstoff, was auch im Raman-Spektrum zu beobachten ist. 


\subsubsection{Raman-spektroskopische $\lambda$-Wert-Bestimmung}

Wird ein Motor gezündet betrieben, so werden die Abgase in der Auslassphase eines Motorzykluses nicht vollständig ausgestoßen und es verbleiben dadurch Verbrennungsreste im Brennraum. Da aber der Versuchsmotor bei allen Messungen geschleppt betrieben und keine Abgase erzeugt wurden, kann das Stickstoff- zu Sauerstoffverhältnis als konstant angenommen werden. Aus dem Verhältnis der Intensitäten der Raman-Linien des Luft/Kraftstoffgemisches wurde ein ortsaufgelöster Lambda-Wert bestimmt. Um das Intensitätsverhältnis der $\mathrm{N}_{2^{-}}$zur CH-Raman-Linie zu kalibrieren, wurde der Motor homogen betrieben. Der $\lambda$-Wert ist dabei überall im Brennraum gleich und kann in diesem Betriebsmodus aus der eingespritzten Kraftstoffmenge und dem angesaugten Luftmassenstrom berechnet werden. Abbildung 4.18 zeigt drei auf diese Weise gewonnene Profile von Raman-Spektren, die zur Kalibration der ortsaufgelösten Spektren verwendet wurden.

Eine weitere Möglichkeit zur Kalibration besteht darin, den Motor in den verwendeten Betriebspunkten gefeuert zu betreiben und über den Restsauerstoffgehalt im Abgas mit Hilfe einer Lambda-Sonde den Lambda-Wert zu bestimmen.

Mit Hilfe der Kalibration konnte der Lambda-Wert ortsaufgelöst mit Hilfe der RamanSpektren in den geschichteten Betriebspunkten bestimmt werden. Um Abbildungsfehler und Verschmutzungen im optischen Strahlengang außerhalb des Motors aus den Raman-

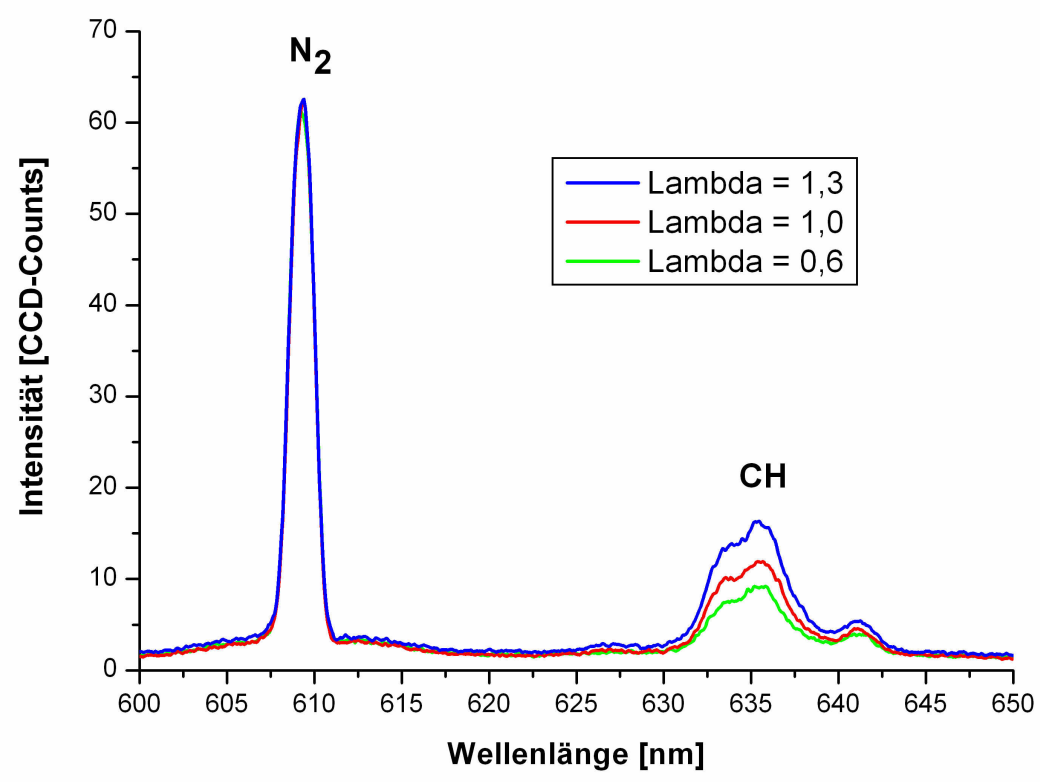

Abbildung 4.18.: Raman-Spektren im Homogenbetrieb zur Kalibration des Lambda-Wertes 
Spektren herausrechnen zu können, wurde zunächst der Motor nur geschleppt und ohne Einspritzung betrieben. Ein hierbei gemitteltes Raman-Spektrum der Ansaugluft wurde zum Normieren der im geschichteten Betrieb aufgenommenen Raman-Spektren verwendet.

$\mathrm{Zu}$ jedem geschichteten Betriebspunkt wurden dann die 100 gemessenen, ortsaufgelösten Raman-Spektren gemittelt und kalibriert. Danach wurde zu jedem Ort im Wellenlängenbereich zwischen $615 \mathrm{~nm}$ und 625nm aus dem detektierten Signal ein Hintergrundsignal ermittelt und jeweils vom Stickstoff- und vom Kraftstoff-Raman-Signal abgezogen. Die beiden Signalintensitäten wurden dann über ihre spektrale Breite gemittelt und ergaben so ortsaufgelöste Intensitätsprofile. Anschließend wurden die Ortsprofile der Raman-Linien mit einem gleitenden Mittelwert (Breite: 5 Pixel) geglättet und ins Verhältnis gesetzt. Der aus den Messungen im homogenen Betrieb ermittelte Kalibrationsfaktor (Abbildung 4.18) ermöglichte dann die Umrechnung auf einen ortsaufgelösten Lambda-Wert. Der aus der Photonenstatistik der Raman-Signale berechnete relative Fehler lag im Mittel bei etwa $10 \%$.

Beispielhaft sind in den oberen Graphen der Abbildung 4.19, 4.20 und 4.21 die so gewonnenen Lambda-Wert-Verteilungen der drei untersuchten Einspritzventile bei einem Betriebspunkt ( $\mathrm{ASE}=30^{\circ} \mathrm{KWvOT}$, Raildruck=100bar) zu verschiedenen Aufnahmezeiten (Kurbelwinkel vor ZOT) dargestellt. Zu frühen Zeitpunkten nach dem Ansteuerende der Einspritzventile, bei denen ein starker, flüssiger Hohlkegel des Sprays ausgebildet war, verursachte das auf die Tröpfchen des mit dem Farbstoff versetzten Euro Super auftreffende Laserlicht des Nd:YAG-Lasers eine sehr starke breitbandige Emission, die wahrscheinlich von Gasdurchbrüchen herrührte und das Raman-Signal überlagerte. Eine Auswertung dieser Aufnahmen war dadurch unmöglich. Erst ab etwa $9^{\circ} \mathrm{KW}$ nach dem ASE konnte aus den Raman-Spektren jeweils eine ortsaufgelöste Lambda-Wert-Verteilung bestimmt und damit eine zeitliche Entwicklung dargestellt werden.

Zusätzlich zu dem $\lambda$-Wert wurde aus den gemittelten 100 einzelnen Raman-Spektren eines Aufnahmezeitpunktes ein Schwellwert des Kraftstoff-Raman-Signals bestimmt, ab dem man von der tatsächlichen Anwesenheit von Kraftstoff an einem Ort ausgehen kann. Wird dieser Schwellwert an einem Ort in einer Einzelaufnahme überschritten, so wird dieser mit 1 bewertet, ansonsten mit 0. Auf diese Weise erhält man aus den 100 Spektren für jede Position eine Wahrscheinlichkeit für das Auftreten von Kraftstoff. Dadurch lassen sich zündsichere Positionen mit geringer Fluktuation finden. In den Abbildungen 4.19, 4.20 und 4.21 unten sind die Kraftstoffwahrscheinlichkeitsverteilungen der drei Injektoren bei dem entsprechenden Betriebspunkt dargestellt. Beispielsweise trat beim Ringspaltventil (Abbildung 4.21) 20mm vom Einspritzventil entfernt zu frühen Zeiten nach Ansteuerende $\left(21^{\circ} \mathrm{KWvOT}\right)$ noch kein Kraftstoff auf. Doch $12^{\circ} \mathrm{KW}$ nach ASE, also ab $9^{\circ} \mathrm{KWvOT}$, ist Kraftstoff hier fast immer vorhanden. Diese Struktur wird im LIF-Bild als äußerer Ring sichtbar (siehe Abbildung 4.16). 

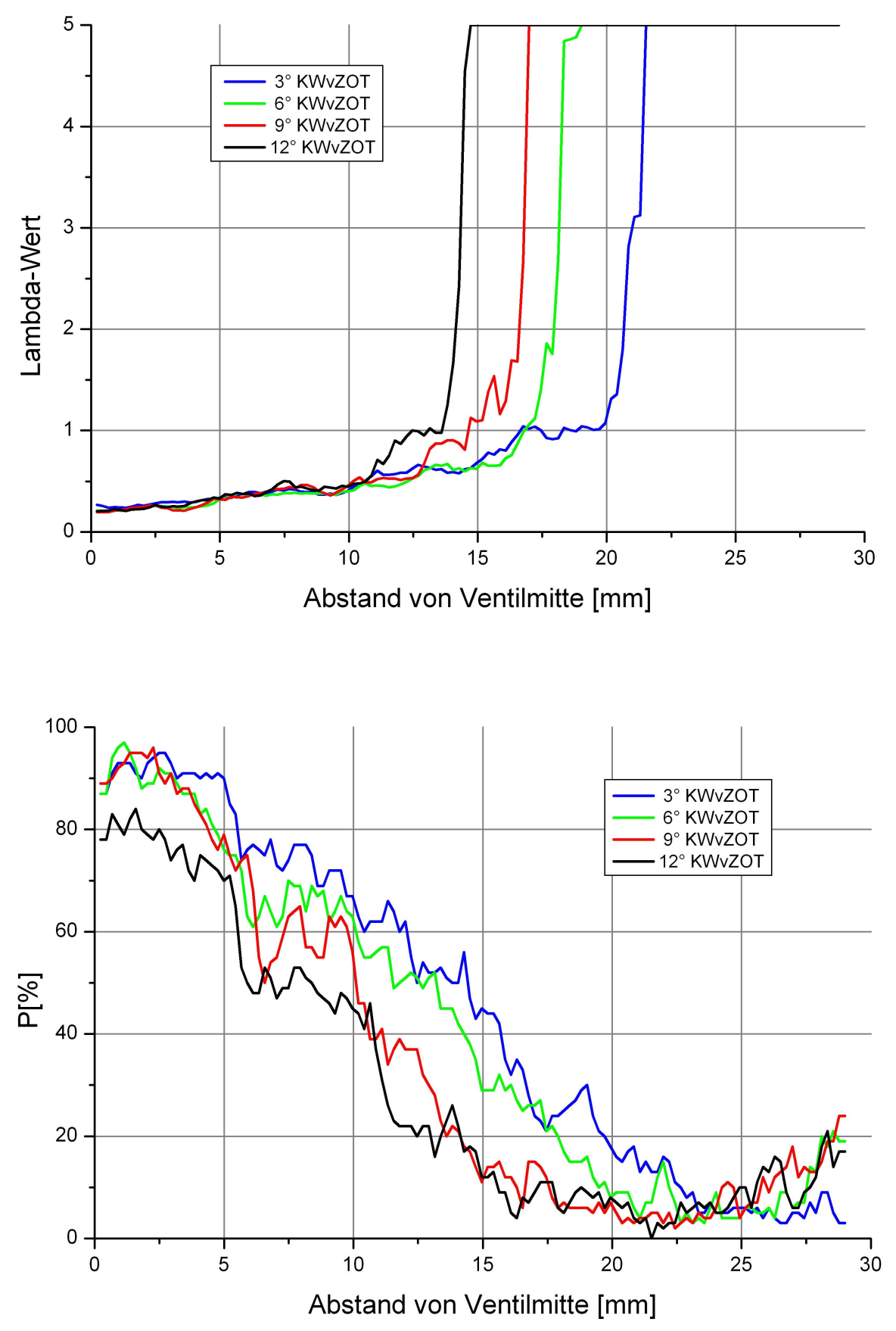

Abbildung 4.19.: Beispiel eines Ergebnisses der Raman-Messungen für das Drallventil - oben dargestellt ist die ortsaufgelöste Lambda-Wert-Verteilung und unten die Kraftstoffwahrscheinlichkeitsverteilung 

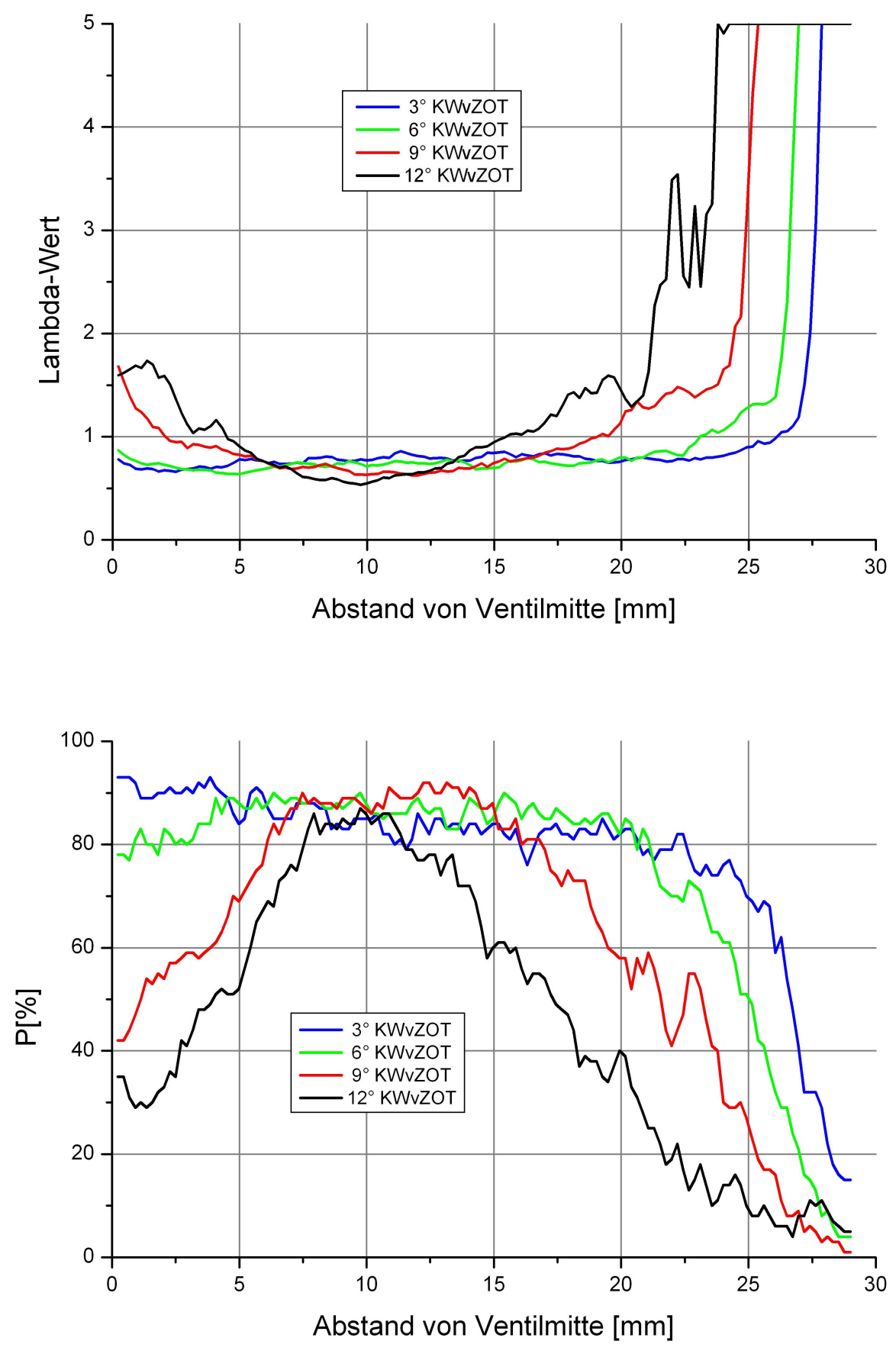

Abbildung 4.20.: Beispiel eines Ergebnisses der Raman-Messungen für das Mehrlochventil - oben dargestellt ist die ortsaufgelöste Lambda-Wert-Verteilung und unten die Kraftstoffwahrscheinlichkeitsverteilung 

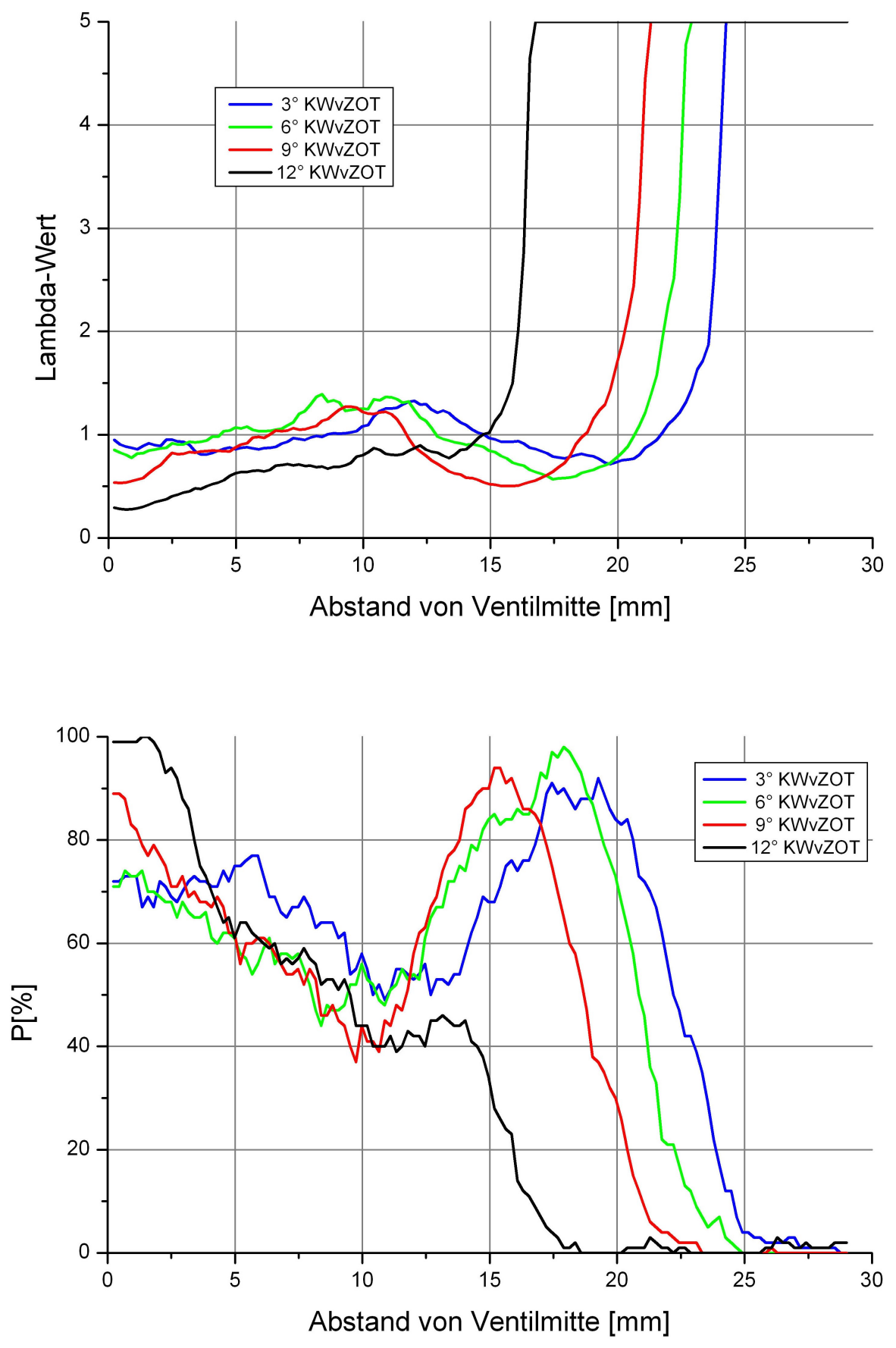

Abbildung 4.21.: Beispiel eines Ergebnisses der Raman-Messungen für das Ringspaltventil - oben dargestellt ist die ortsaufgelöste Lambda-Wert-Verteilung und unten die Kraftstoffwahrscheinlichkeitsverteilung 


\subsubsection{Zusammenhang zwischen Raman- und LIF-Messungen}

In Abschnitt 3.2 wurde die Fluoreszenzlöschung der Kraftstoff-Fluoreszenz durch Sauerstoff beschrieben. Dieser Prozess kommt erst bei hohen Drücken, also zu späten Zeitpunkten der Kompression des Motors zum tragen. Um die Möglichkeit einer flächigen LambdaWert-Bestimmung mittels laserinduzierter Fluoreszenz (LIF) zu untersuchen, wurden die Intensitätsprofile der deckungsgleichen Messvolumina aus den LIF-Messungen mit den dazugehörenden Raman-Signalen verglichen (siehe Abbildung 4.22). Eine Korrelation der beiden Profile gibt einen Aufschluss darüber, zu welchen Zeitpunkten eine Kalibration des flächigen LIF-Signals mit der aus den Raman-Messungen gewonnenen ortsaufgelösten Lambda-Wert-Verteilung möglich wäre.

Beispielhaft ist in Abbildung 4.23 für die Einspritzung mit dem Mehrlochventil zu einem Betriebspunkt (ASE $=30^{\circ} \mathrm{KWvOT}$, Raildruck=100bar) die zeitliche Entwicklung der beiden Intensitätsprofile während der Kompression dargestellt. Zu frühen Zeitpunkten $\left(24^{\circ} \mathrm{KWvOT}\right.$ und $\left.21^{\circ} \mathrm{KWvOT}\right)$ sind die Verläufe der LIF- und der Raman-Signalprofile noch sehr unterschiedlich. Erst zu späten Zeitpunkten $\left(18^{\circ} \mathrm{KWvOT}\right.$ und $\left.15^{\circ} \mathrm{KWvOT}\right)$ ist der Kraftstoff weitestgehend verdampft und der Zylinderinnendruck so hoch, dass die durch das Quenching des Sauerstoffs beeinflusste Fluoreszenzintensität dem RamanSignal-Verlauf ähnlich ist. Um eine flächige Lambda-Wert-Verteilung zu bestimmen, wäre

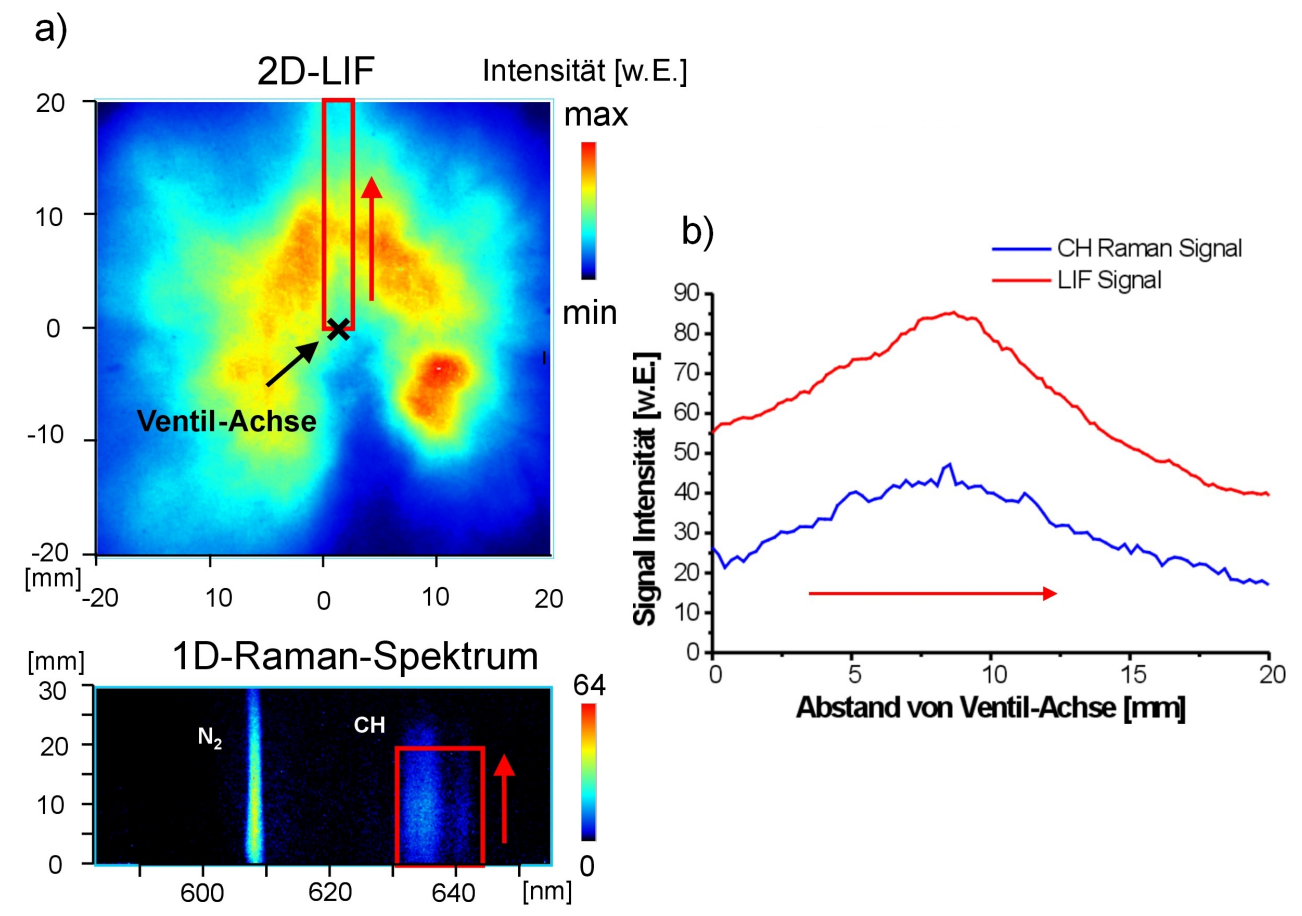

Abbildung 4.22.: Vergleich der Intensitätsprofile deckungsgleicher Messvolumina (rot umrandet) in gemittelten LIF- und Raman-Messungen 


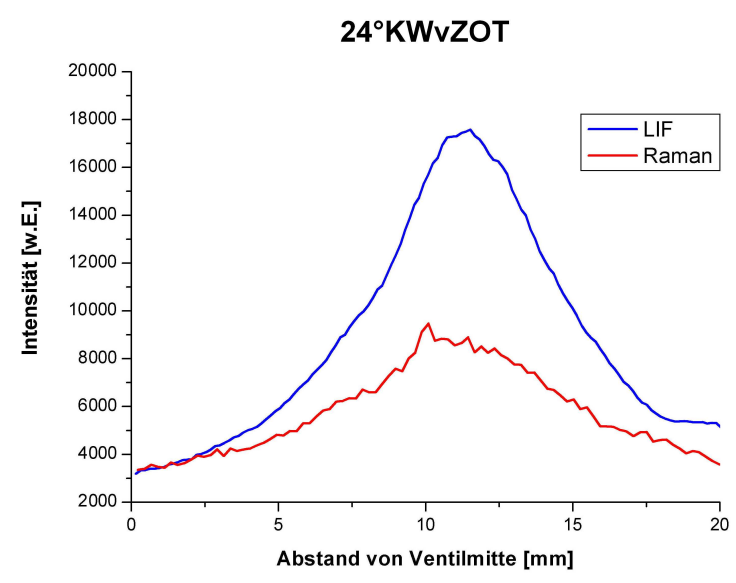

$18^{\circ} \mathrm{KWvZOT}$

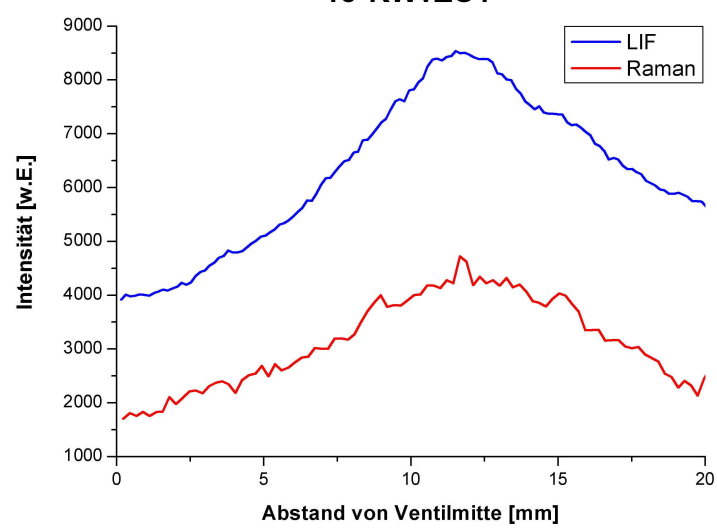

$21^{\circ} \mathrm{KWvZOT}$

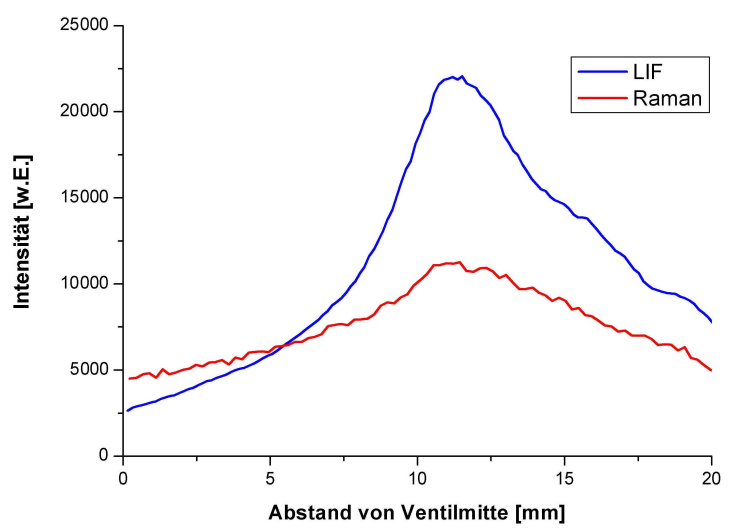

$15^{\circ} \mathrm{KWvZOT}$

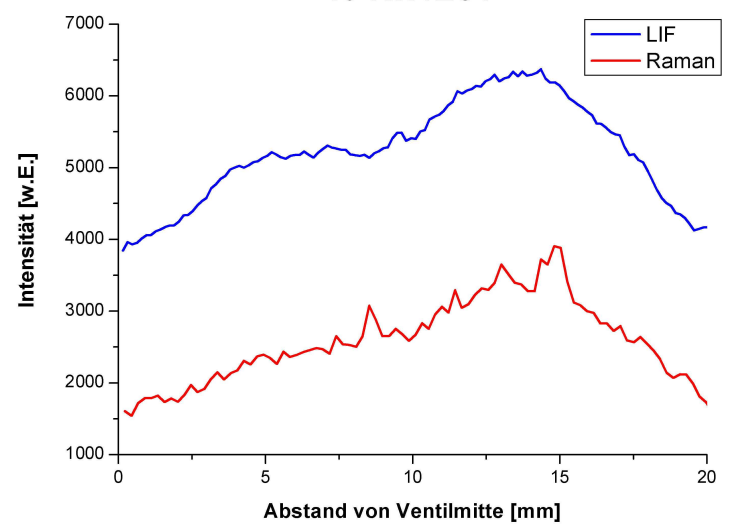

Abbildung 4.23.: Zeitliche Entwicklung der LIF- und Raman-Signalintensitäten beim Mehrlochventil (Ansteuerende (ASE) des Injektors ist $30^{\circ} \mathrm{KWvOT}$ bei $100 \mathrm{bar}$ Raildruck)

eine Kalibration des LIF-Signals also nur zu diesen Zeitpunkten möglich. Allerdings ist zu beachten, dass die Absorption des Kraftstoffs den anregenden UV-Laserstrahl abschwächt und so die Fluoreszenzintensität an Orten gleicher Kraftstoffdichte nicht identisch ist. Dieses müsste rechnerisch durch iterative Prozesse korrigiert werden. 


\subsection{Faseroptisches Sensorsystem}

Eine Optimierung benzindirekteinspritzender Brennverfahren setzt die genaue Kenntnis über die zeitliche Entwicklung des $\lambda$-Wertes an der Zündkerze voraus, da Parameter wie Einspritzdruck, -dauer und -zeitpunkt einen Einfluss darauf haben, ob und wann der eingespritzte Kraftstoff zündfähig ist. Dieses wurde schon bei den in Abschnitt 4.3 beschriebenen motorischen Untersuchungen deutlich. Um eine Gemischbildungsanalyse nicht nur in einem für Forschungszwecke präparierten Transparentmotor, sondern auch in einem nichtmodifizierten Serienmotor durchführen zu können, sollte daher ein faseroptischer Sensor entwickelt werden, der anstelle der Zündkerze in den Motor eingesetzt werden kann und dort mittels spontaner Raman-Streuung das Luft/Kraftstoffverhältnis misst.

In diesem Abschnitt werden neben dem Aufbau des entwickelten Raman-Sensors auch die damit erfolgten motorischen Untersuchungen an dem im vorhergehenden Abschnitt beschriebenen Transparentmotor dargestellt.

\subsubsection{Eigenschaften von Glasfasern bei hoher Laserleistung}

Durch den im Vergleich zur UV-Anregung geringeren Raman-Streuquerschnitt bei 532nm musste zunächst untersucht werden, was bei der Verwendung von Glasfasern beim Transport hoher Lichtleistungen zu beachten ist. Dazu wurde das Strahlprofil des Nd:YAGLasers mit Hilfe des Homogenisators (Abschnitt 4.1.1) homogenisiert und anschließend mit einer an die NA der verwendeten Glasfaser von 0.22 angepassten Linse in diese eingekoppelt. Der Versuchsaufbau ist in Abbildung 4.24 skizziert. Im sichtbaren Wellenlängenbereich werden üblicherweise Glasfasern mit hohem OH-Gehalt gewählt, da diese eine geringe Dämpfung des transmittierten Lichtes aufweisen. Die hier verwendete $11 \mathrm{~m}$ lange Glasfaser (3M Optical Fiber - FG-550-UER) besaß eine Dämpfung von etwa 10dB/km bei $532 \mathrm{~nm}$ und einen Kerndurchmesser von $550 \mu \mathrm{m}$. Das aus der Faser austretende Laserlicht wurde mit einer kurzbrennweitigen Linse fokussiert und das im Fokus entstandene Raman-Streulicht senkrecht dazu auf den Eintrittsspalt des Spektrographen abgebildet. Zur Signaldetektion wurde der in Abschnitt 4.1.2 vorgestellte Channel Photomultiplier verwendet und das Gitter des Spektrographen in 1nm Schritten gedreht, um die Zentralwellenlänge zu variieren. Zu jedem Schritt wurde das Signal des Multipliers aufgezeichnet und über 16 Laserpulse gemittelt, so dass ein vollständiges Spektrum entstand.

Die durch die Glasfaser transmittierte Pulsenergie betrug 14mJ. Das Spektrum des im Messvolumen entstandenen Streulichtes ist in Abbildung 4.25 gezeigt. Durch die hohe Energiedichte im Kern der Faser entsteht durch stimulierte Raman-Streuung (SRS) Stokes-Strahlung an den $\mathrm{SiO}_{2}$-Molekülen des Glases mit einer Frequenzverschiebung von $440 \mathrm{~cm}^{-1}$ [56]. Daraus resultiert die 1. Stokes-Linie bei etwa 545nm. Durch die hohe Streulichtintensität bei dieser Wellenlänge wurde die Sättigung des Channel Multiplier erreicht, so dass das eigentliche Raman-Signal größer ist, als hier dargestellt. Da die 1. Stokes-Linie wieder eine sehr große Energiedichte in der Glasfaser erzeugte, entstand ebenfalls durch 


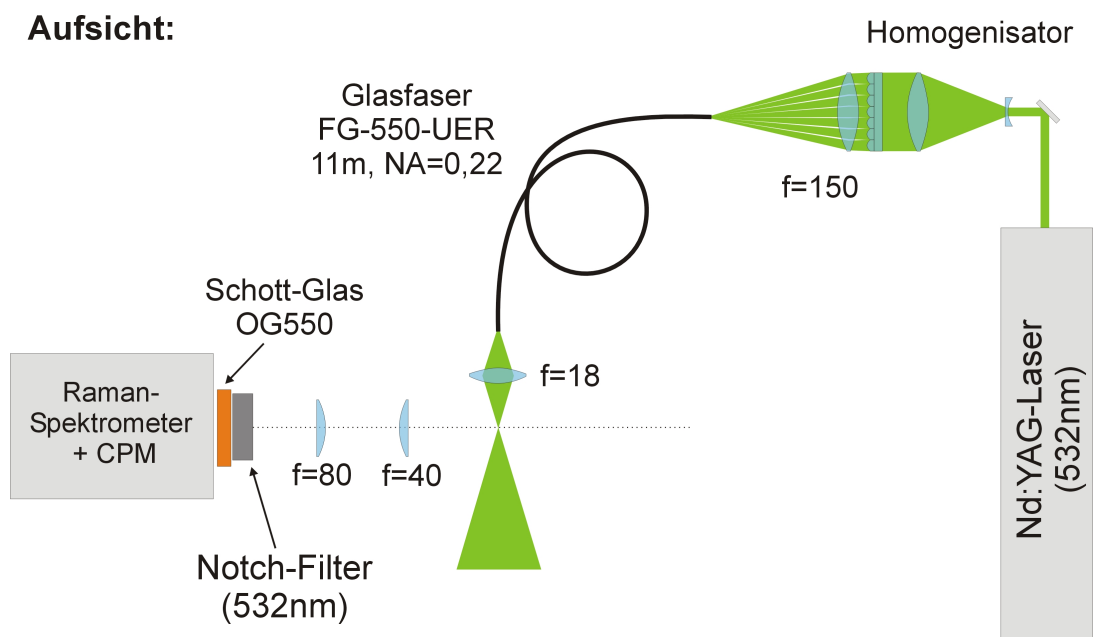

Abbildung 4.24.: Experimenteller Aufbau zur Untersuchung der Einkoppeleigenschaften von Glasfasern

stimulierte Raman-Streuung eine Stokes-Strahlung 2. Ordnung bei etwa 558nm. Im Spektrum ist erkennbar, dass auf diese Weise in der Glasfaser Stokes-Strahlung bis zur 8. Ordnung entstand. Allerdings ist sie hier aufbaubedingt nur sichtbar, da sie an Luftmolekülen Rayleigh-gestreut und so auf den Spektrographen abgebildet wurde.

Eine genauere Untersuchung des Spektrums zeigte, dass weitere Spektrallinien durch Raman-Streuung im Messvolumen erzeugt wurden, wie in Abbildung $4.26 \mathrm{zu}$ sehen ist. Neben der durch SRS im Glas erzeugten Stokes-Strahlung erfolgte Raman-Streuung des ursprünglich eingestrahlten Lichtes des Nd:YAG-Lasers bei 532nm an Raumluft. Es sind Vibrations-Ramanlinien von Sauerstoff bei $585 \mathrm{~nm}$ und von Stickstoff bei $608 \mathrm{~nm}$ erkennbar. Die durch die $\mathrm{SiO}_{2}$-Moleküle bei 545nm entstandene Stokes-Strahlung 1. Ordnung ist so stark, dass die Raman-Streuung an $\mathrm{O}_{2}$ - und $\mathrm{N}_{2}$-Molekülen der Raumluft ebenfalls sichtbar ist.

Das Ergebnis dieser Untersuchung war, dass die Vibrations-Ramanlinie einer CH-Bindung bei $635 \mathrm{~nm}$ keine Überlagerung durch andere Raman-Linien besitzt, aber sowohl Stickstoff als auch Sauerstoff mit $\mathrm{SiO}_{2}$-Linien überlappen. Dieses würde eine Bestimmung des $\lambda$ Wertes erschweren. Je länger eine Glasfaser ist, desto länger ist die Wechselwirkungslänge des Laserlichts mit dem Glas und um so niedriger liegt die Schwelle für stimulierte RamanStreuung [3]. Um diese bei hohen Laserleistungen anzuheben, sollte ein optischer Sensor mit möglichst kurzen Glasfasern ausgestattet sein. Zusätzlich lässt sich die Leistungsdichte in ihrem Inneren durch die Verwendung größerer Faserkerne senken.

Eine zusätzliche Möglichkeit besteht darin, das Laserlicht nicht nur in eine einzelne Glasfaser, sondern in ein ganzes Bündel davon einzukoppeln. Es hat sich allerdings in den Experimenten gezeigt, dass Bündel von Fasern eher durch die hohen Leistungsdichten zerstört wurden, als dies bei einer einzelnen der Fall war. Grund dafür ist die Überstrahlung der Faserendflächen, d.h., nicht nur im Kern, sondern auch im Mantel der Fasern ist die Leistungsdichte des Laserlichts hoch. Die größere Absorption durch die eingelagerten Zusatzstoffe (siehe Abschnitt 2.2) dort senkt die Zerstörschwelle. 


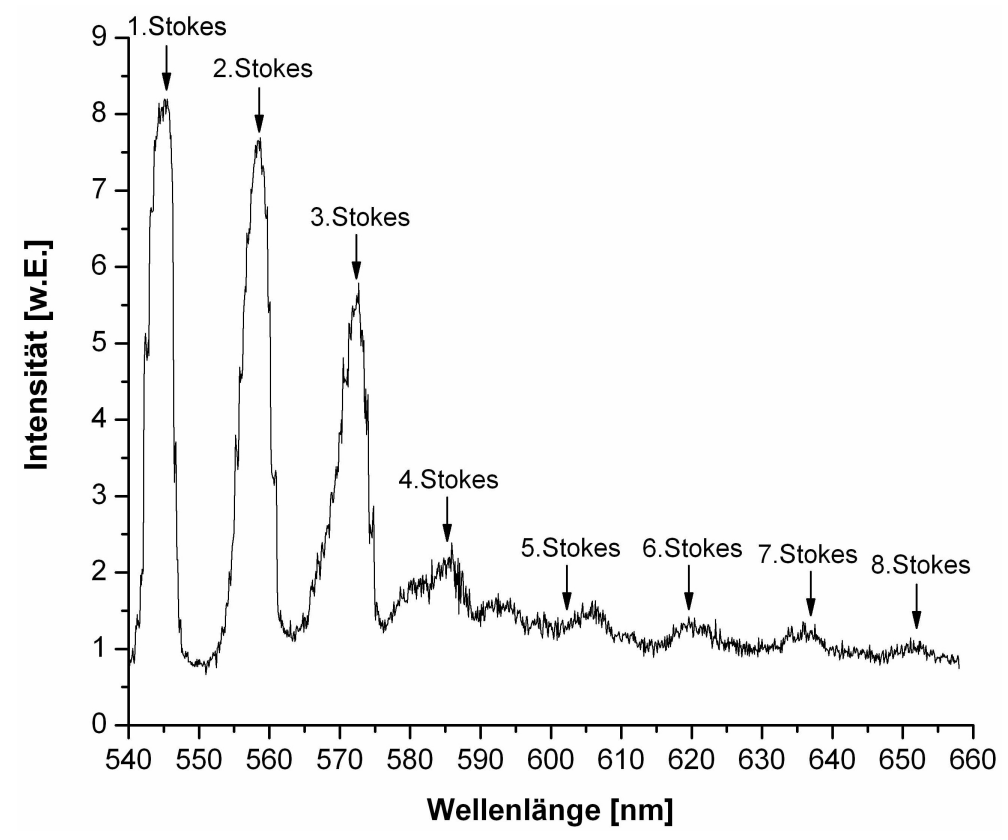

Abbildung 4.25.: In einer Glasfaser durch SRS erzeugte Raman-Linien verschiedener Ordnung

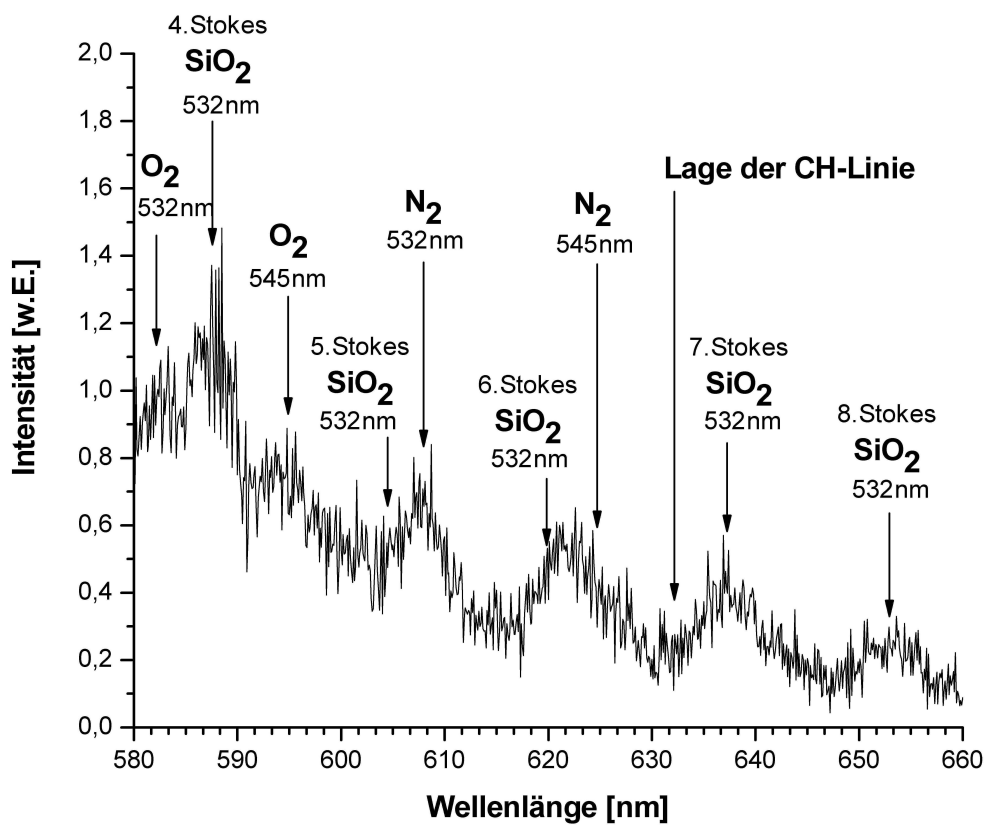

Abbildung 4.26.: Spektrum der im Messvolumen erzeugten Streuung. Angegeben ist, woran die RamanStreuung erfolgte und durch welche Wellenlänge. 


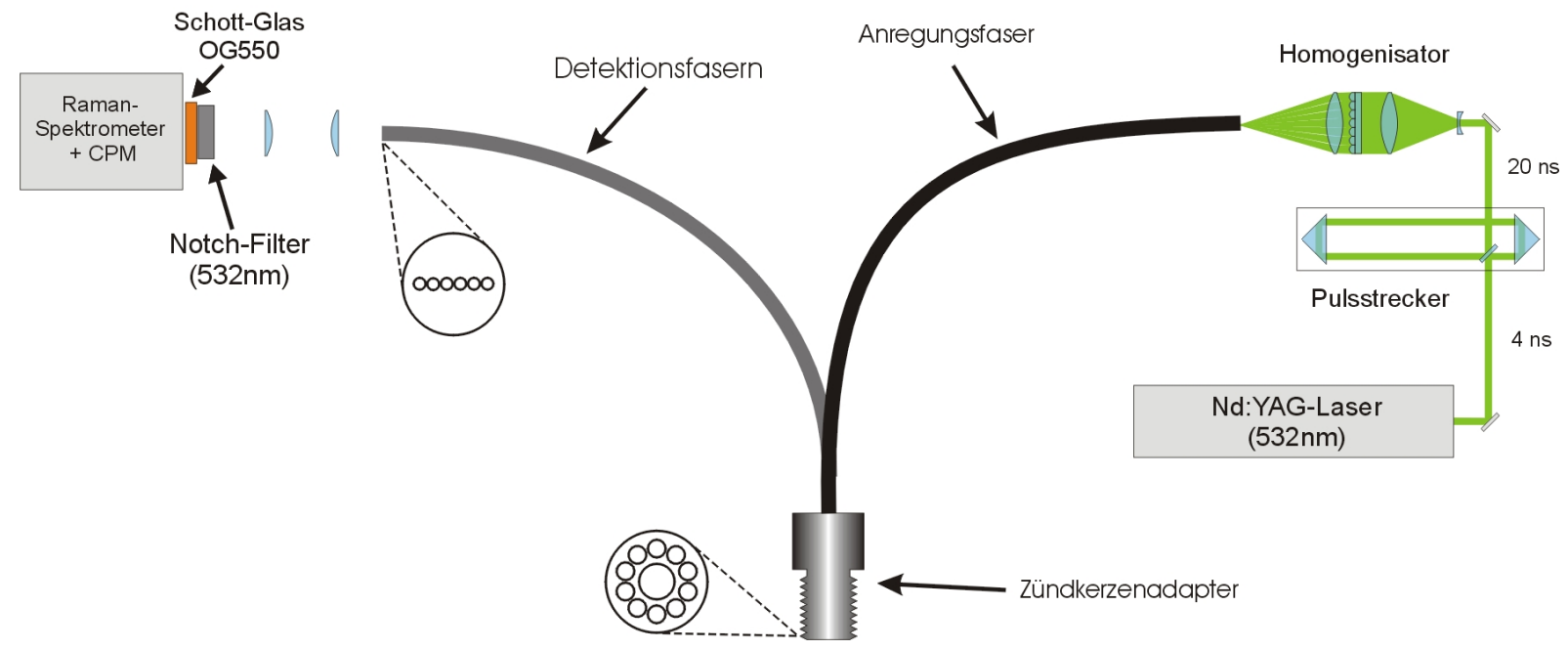

Abbildung 4.27.: Experimenteller Aufbau des faseroptischen Raman-Sensors

\subsubsection{Aufbau des faseroptischen Raman-Sensors}

Die gewonnenen Erkenntnisse über die Eigenschaften von Glasfasern lieferten einen großen Beitrag zur Konzeption eines faseroptischen Raman-Sensors für den Einsatz an einem Ottomotor. Der Aufbau des Sensors ist in Abbildung 4.27 skizziert. Das Laserlicht des Nd:YAG-Lasers durchlief zunächst den Pulsstrecker und den Homogenisator (Abschnitt 4.1.1). Anschließend wurde es in eine 2m lange Glasfaser (Fiberguide - XPS1500) fokussiert, die einen Kern von 1,5mm Durchmesser besaß. Durch diese Faser konnte das Licht des Lasers mit einer Pulsenergie von bis zu $75 \mathrm{~mJ}$ zerstörungsfrei in den Brennraum eines Motors geleitet werden. Bei diesen hohen Energien allerdings war die Leistungsdichte im Kern der Faser so hoch, dass stimulierte Raman-Streuung auftrat. Aus diesem Grund wurden alle weiteren Messungen mit deutlich weniger Lichtleistung gemacht. Der Zündkerzenadapter des Sensors besaß die Abmessungen einer Zündkerze und konnte wie diese eingeschraubt werden. Um möglichst viel Raman-gestreutes Licht aufsammeln zu können, war die große Anregungsfaser von 10 Glasfasern umgeben, die einen $600 \mu \mathrm{m}$ großen Kern hatten. Die 10 Detektionsfasern wurden auf der Auskopplungsseite nebeneinander angeordnet. So konnte das Licht aus den Fasern optimal auf dessen Eintrittsspalt abgebildet werden. Genau wie bei den in Abschnitt 4.3 beschriebenen motorischen Untersuchungen wurde mit der bildverstärkten CCD-Kamera das erzeugte Spektrum aufgenommen. 


\subsubsection{Motorische Untersuchungen}

Mit dem faseroptischen Raman-Sensor wurden ebenfalls Untersuchungen am Glasringmotor vorgenommen. An Stelle des Einspritzventils (siehe Abbildung 4.14) wurde der Fasersensor eingesetzt. Die Einspritzung des Kraftstoffes erfolgte durch ein an der Seite des Brennraumdaches plaziertes Drallventil.

Im Spektrum der durch den Fasersensor erzeugten und detektierten spontanen RamanStreuung zeigten sich deutlich von den einzelnen Detektionsfasern verursachte spektral breite Fluoreszenzen (Abbildung 4.28). Ursache hierfür ist die hohe Laserleistungsdichte in der zentralen Anregungsfaser. Im Zündkerzenadapter waren die Glasfasern von ihrer Schutzummantelung befreit und mit einem durch Hitze aushärtenden Optikkleber eingeklebt. Da der Brechungsindex im Kleber ähnlich dem der Faser ist und so die Lichtleitung durch fehlende Totalreflexion nicht mehr auf die Glasfaser beschränkt war, konnte Licht aus der Anregungsfaser in die Detektionsfasern überkoppeln. Aus der Literatur ist bekannt, dass Farbzentren, welche beim Herstellungsprozess von Glasfasern durch Defekte in der Kristallstruktur der $\mathrm{SiO}_{2}$-Bindungen entstehen, und im Mantelbereich (Cladding) zur Dotierung eingelagertes Fluor Ursache von spektral breiten Fluoreszenzen bei starker Bestrahlung mit Laserlicht sind [30, 28]. Die in der Anregungsfaser transmittierte Pulsenergie des Laserlichts betrug bei allen motorischen Untersuchungen etwa $45 \mathrm{~mJ}$.

Wie sich bereits bei den Voruntersuchungen am Kraftstoffspray (Abschnitt 4.2.3) gezeigt hatte, verursachte das mittels Glasfaser in den Motor transmittierte Laserlicht in den Tröpfchen des eingespritzten Kraftstoffes stimulierte Raman-Streuung. Dies führte zu einer Überhöhung des Kraftstoff-Raman-Signals (Abbildung 4.29). Deshalb war es auch beim Einsatz des faseroptischen Raman-Sensors notwendig, dem Kraftstoff Euro Super farblos den Absorberfarbstoff Sudanrot beizumengen.

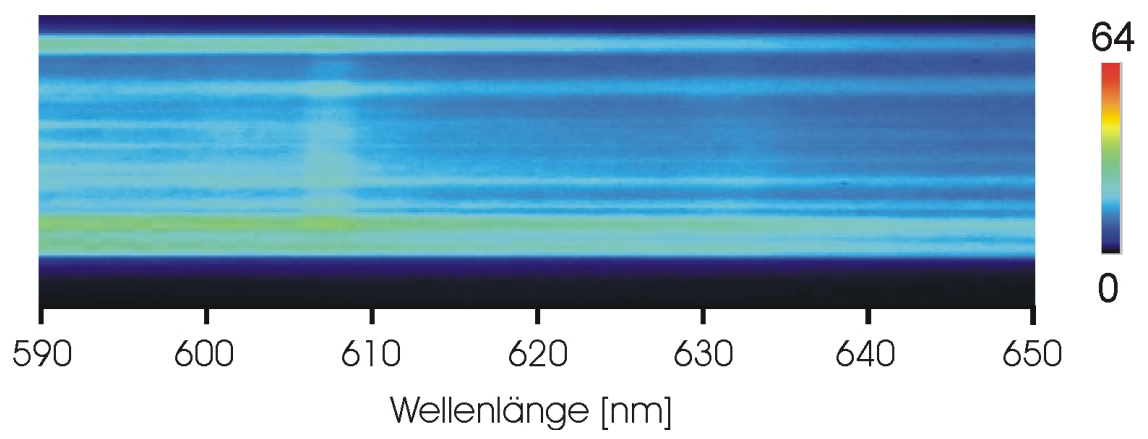

Abbildung 4.28.: Das mit Hilfe des Fasersensors im Motor gewonnene Spektrum der spontanen RamanStreuung zeigt deutlich die von den einzelnen Glasfasern verursachte spektral breite Fluoreszenz 


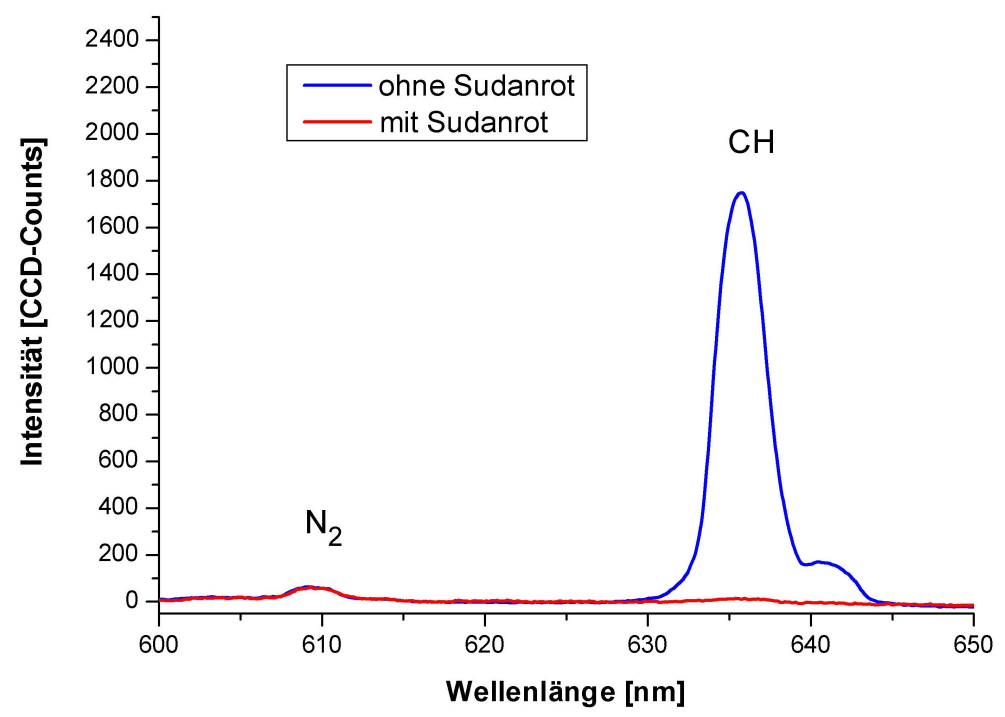

Abbildung 4.29.: Bei hoher Laserleistung mit dem faseroptischen Raman-Sensor gemessene RamanSpektren eines Kraftstoffgemischs mit und ohne Absorberfarbstoff

Zunächst wurden Messungen am Transparentmotor im homogenen Betrieb vorgenommen. Dabei erfolgte keine Kalibration des Luft/Kraftstoffverhältnisses zum LambdaWert. In Abbildung 4.30 sind die mit Hilfe des Fasersensors angeregten und detektierten Raman-Spektren für verschiedene Einspritzdauern des Injektors im Homogenbetrieb dargestellt. Die Spektren wurden über 400 Einzelschüsse gemittelt und anschließend der Fluoreszenzuntergrund abgezogen. Neben den Stickstoff- und Kraftstoff-RamanLinien traten im Spektrum weitere Linien auf. Diese sind, wie in Abschnitt 4.4.1 gezeigt, durch Raman-Streuung am $\mathrm{SiO}_{2}$ in der Anregungsfaser erzeugte Raman-StokesLinien 5. Ordnung, die die Stickstoff-Linie überlagern. Die Bestimmung eines exakten Luft/Kraftstoffverhältnisses aus den Signalen war dadurch nicht möglich. Dennoch ließ sich ein Verlauf dieses Verhältnisses am Ort der Zündkerze näherungsweise bestimmen (siehe Abbildung 4.31). Das Ansteuerende des Einspritzventils lag bei $42^{\circ} \mathrm{KWvOT}$.

Somit wurde gezeigt, dass die Zündkerzenbohrung eines Motors als einziger optischer Zugang ausreicht, um mit Hilfe der spontanen Raman-Streuung den Lambda-Wert eines Gemisches im Brennraum zu bestimmen. Allerdings war es bei den Messungen notwendig, über viele Einzelschüsse zu mitteln, da die einzelnen Aufnahmen zu verrauscht und nicht auswertbar waren. Im Gegensatz zu den Freistrahlexperimenten war es damit nicht möglich eine Statistik der Zyklusschwankungen anzugeben. Die genaue Kenntnis dieser Schwankungen geben allerdings erst einen Hinweis auf die tatsächlichen Gemischbildungsvorgänge. 


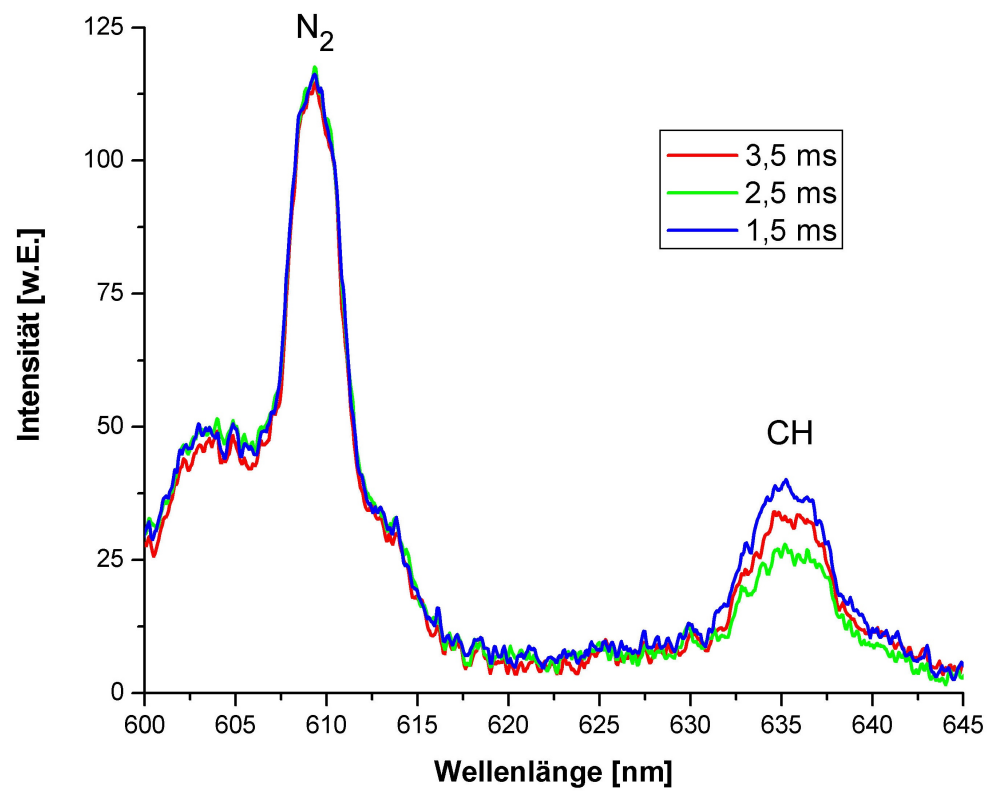

Abbildung 4.30.: Durch den Fasersensor angeregte und detektierte Raman-Spektren (gemittelt über 400 Einzelschüsse) für versch. Einspritzdauern des Injektors im Homogenbetrieb

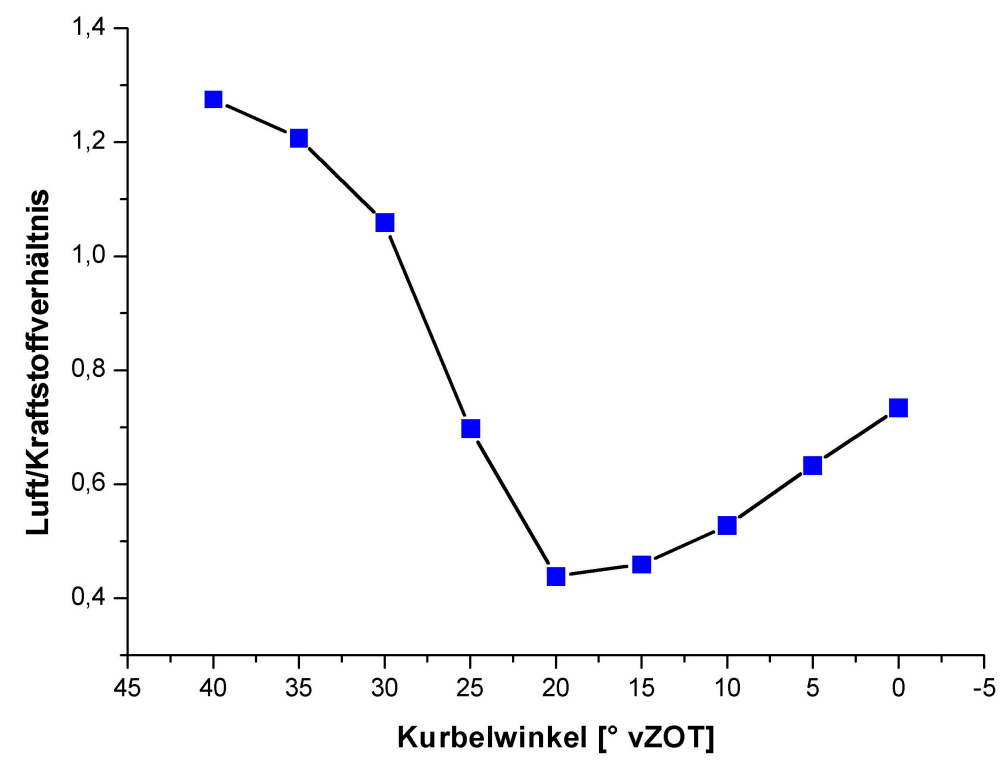

Abbildung 4.31.: Verlauf des angenäherten Luft/Kraftstoffverhältnisses am Ort der Zündkerze $\left(\mathrm{ASE}=42^{\circ} \mathrm{KWvOT}, 100 \mathrm{bar}\right.$ Raildruck) 


\subsection{Neue Detektionskonzepte}

Die ersten motorischen Untersuchungen mit dem vorgestellten faseroptischen RamanSensor haben gezeigt, dass die Bestimmung des Lambda-Wertes an der Zündkerze mit dieser Technik prinzipiell möglich ist. Allerdings war das Signal/Rausch-Verhältnis der Einzelaufnahmen nicht ausreichend, um zyklische Schwankungen der Gemischbildung bestimmen zu können. Um dieses zu ermöglichen, kommt aber eine Erhöhung der eingestrahlten Laserenergie nicht in Frage. Wie bereits gezeigt, führen kurze Pulsdauern und inhomogene Strahlprofile leicht zu Beschädigungen optischer Komponenten im Strahlengang. Für eine Optimierung des Messsystems sind daher neben einer effizienteren SignalSammeloptik eine Minimierung der Übertragungsverluste der Messsignale und eine Maximierung der Detektorempfindlichkeit notwendig. Im folgenden Abschnitt werden daher die weiteren Entwicklungsschritte, die zu einer Verbesserung des Signal/Rausch-Verhältnisses führen, dargestellt.

\subsubsection{Sensorgeometrie}

Bei einem rein faseroptischen Raman-Sensor ist es unbedingt notwendig, dass es keine Möglichkeit eines direkten Übersprechens des Laserlichtes aus der Anregungs- in die Detektionsfasern gibt. Die in Abschnitt 4.4.3 gezeigten spektral breiten Fluoreszenzen, welche im Optikkleber und im Cladding der Glasfasern entstanden, lassen sich durch eine Ummantelung der Fasern mit einer optisch undurchlässigen Beschichtung vermeiden. Beispielsweise wäre es möglich den standardmäßig vorhandenen Schutzmantel (Buffer) bis zur Faserspitze beizubehalten. Allerdings würde sich dadurch der Abstand der Glasfasern zueinander vergrößern, was zu einer geringeren Überlappung von Anregungs- und Nachweiskegel der Fasern führt [20]. Eine Möglichkeit die Sammeleffizienz der Detektionsfasern dennoch zu verbessern, ist ein Anschrägen der Faserendfläche, um die Überlappung zu vergrößern $[14,6,11,12]$. Darüber hinaus würde die Verwendung einer Laserlichtquelle mit deutlich längerer Pulsdauer die Leistungsdichte im Faserkern deutlich reduzieren und so weniger stimulierte Effekte zur Folge haben. Eine zeitliche Auflösung von einem Grad Kurbelwinkel wäre bei einer Motordrehzahl von 2000U/min noch mit Pulsdauern bis $80 \mu \mathrm{s}$ gewährleistet.

Eine Prinzipskizze einer alternativen Sensorkopfgeometrie ist in Abbildung 4.32 dargestellt. Der Sensorkopf besteht aus zwei voneinander getrennten Kanälen; einem für die Einkopplung des Laserlichts zur Anregung und einem weiteren für die Auskopplung des detektierten Raman-Streulichts. Diese strikte Separierung ist notwendig, um Überlagerungen der Raman-Signale aus dem Messvolumen mit laserinduzierter Streustrahlung im Inneren des Sensorkopfes zu verhindern. Damit Effekte wie die stimulierte Raman-Streuung in Glasfasern (siehe Abschnitt 4.4.1) generell vermieden werden, erfolgt die Strahlführung des Laserlichts zur Anregung konventionell über Spiegel, z.B. einem Spiegelarm. Die Signalübertragung des detektierten Signals ließe sich über eine einzige Faser mit großem Kerndurchmesser realisieren. 


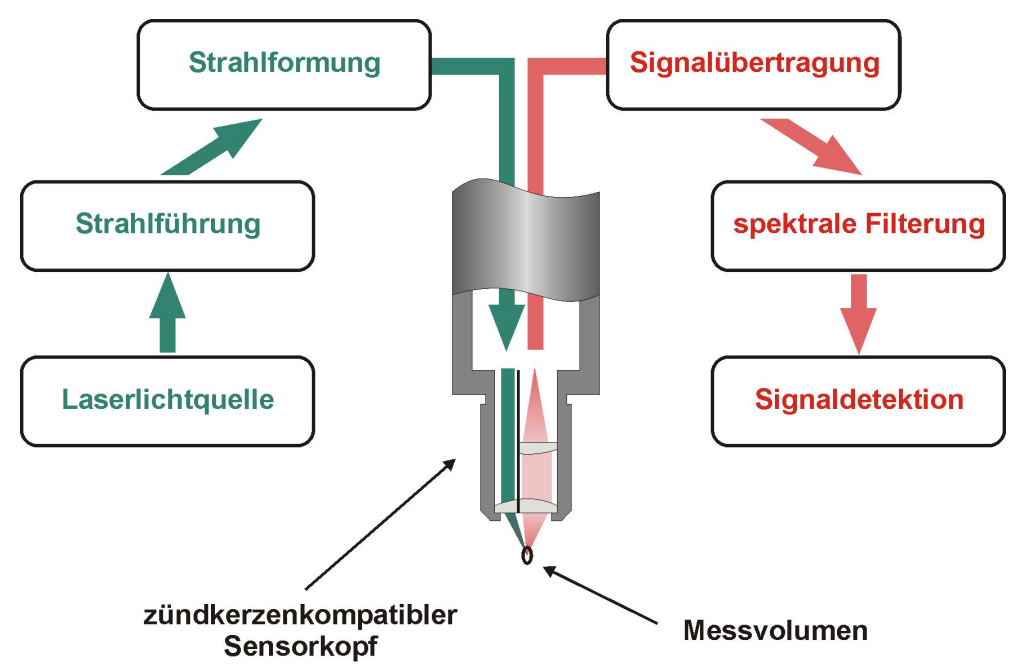

Abbildung 4.32.: Prinzipskizze einer derzeit untersuchten Sensorkopfgeometrie

\subsubsection{Spektrale Filterung der Raman-Signale}

Die spektrale Filterung und Detektion der auftretenden Raman-Signale erfolgte in den vorangegangenen Untersuchungen mit einem Spektrographen und einer bildverstärkten CCD-Kamera (siehe Abschnitt 4.1.2). Um die Entwicklung eines weniger komplexen und kostengünstigeren Messsystems zu ermöglichen, wurden für eine effektive Separation der Raman-Signale spezielle Bandpass-Filter der Firma LOT untersucht. Sie bestehen aus einem Schott-Farbglas als Hochpass-Filter, auf das zusätzlich ein dielektrischer MehrschichtFilter aufgedampft ist. Die Bandbreite beträgt etwa 10nm bei einem maximalen Transmissionsgrad von mehr als 65\%. In Abbildung 4.33 ist beispielhaft die Transmissionskurve des Filters zur Kraftstoffdetektion (LOT - 633FS10-25) gezeigt. Eine Besonderheit dieses Filtertyps ist, dass sich die Zentralwellenlänge der Transmission beim Verkippen des Filters zu kürzeren Wellenlängen hin verschiebt. Außerhalb des transmittierten Spektralbereiches liegt die Reflektivität bei über 99\%. An der Druckkammer erfolgten nun Untersuchungen mit dem bestehenden OMA-System, um die Separationseigenschaften der einzelnen Filter für die Raman-Signale zu testen. Hierzu wurde die Kammer mit einem Luft/Kraftstoffgemisch gefüllt und Raman-Spektren aufgenommen (Abbildung 4.34). Um ein Spektrum des Raman-Streulichtes von Sauerstoff, Stickstoff und Kraftstoff als Referenzspektrum zu erhalten, wurde das elastische Streulicht bei 532nm durch einen vor dem Spektrographen plazierten Hochpass-Filter OG550 unterdrückt. Zur Einzelspeziesdetektion wurde anschließend jeweils ein LOT-Bandpass-Filter unter einem optimierten Winkel vor dem Spektrographen positioniert, so dass eine spektrale Trennung jeweils für Sauerstoff (Filter 580FS10-25), Stickstoff (Filter 610FS10-25) und Kraftstoff (Filter 633FS10-25) erfolgte. 


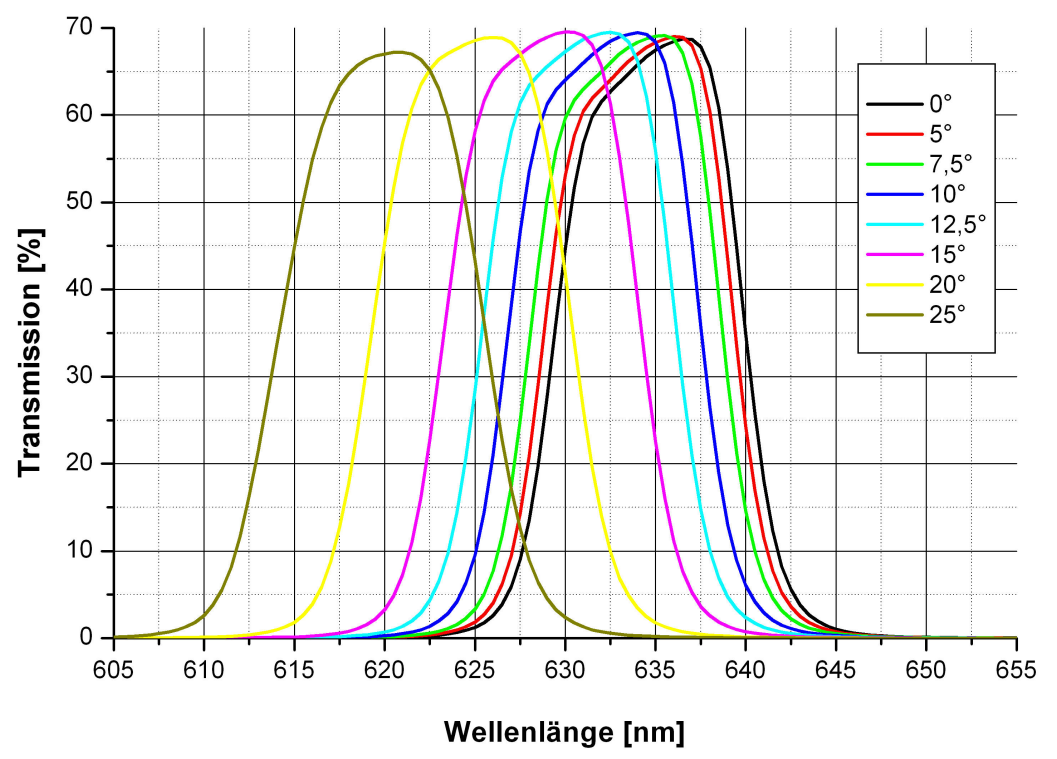

Abbildung 4.33.: Zentralwellenlängenänderung der Transmissionskurve der verwendeten Bandpassfilter unter Verkippung um verschiedene Winkel am Beispiel des Filters zur Kraftstoffdetektion

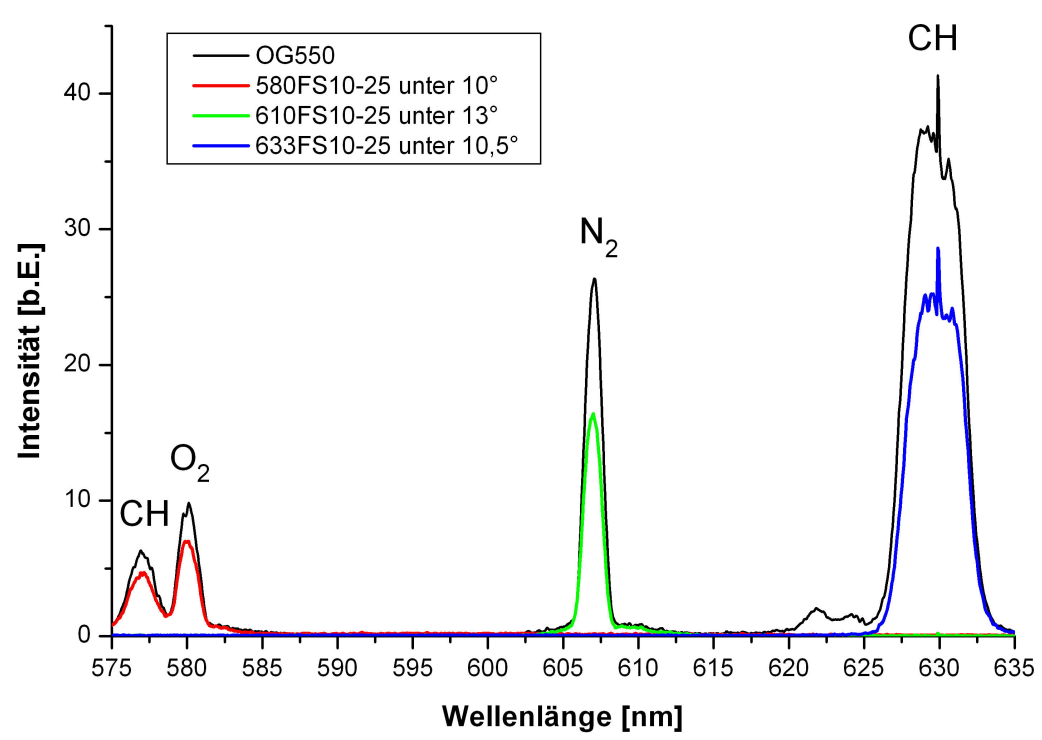

Abbildung 4.34.: Filterwirkung auf Raman-Signale von Sauerstoff, Stickstoff und Kraftstoff 


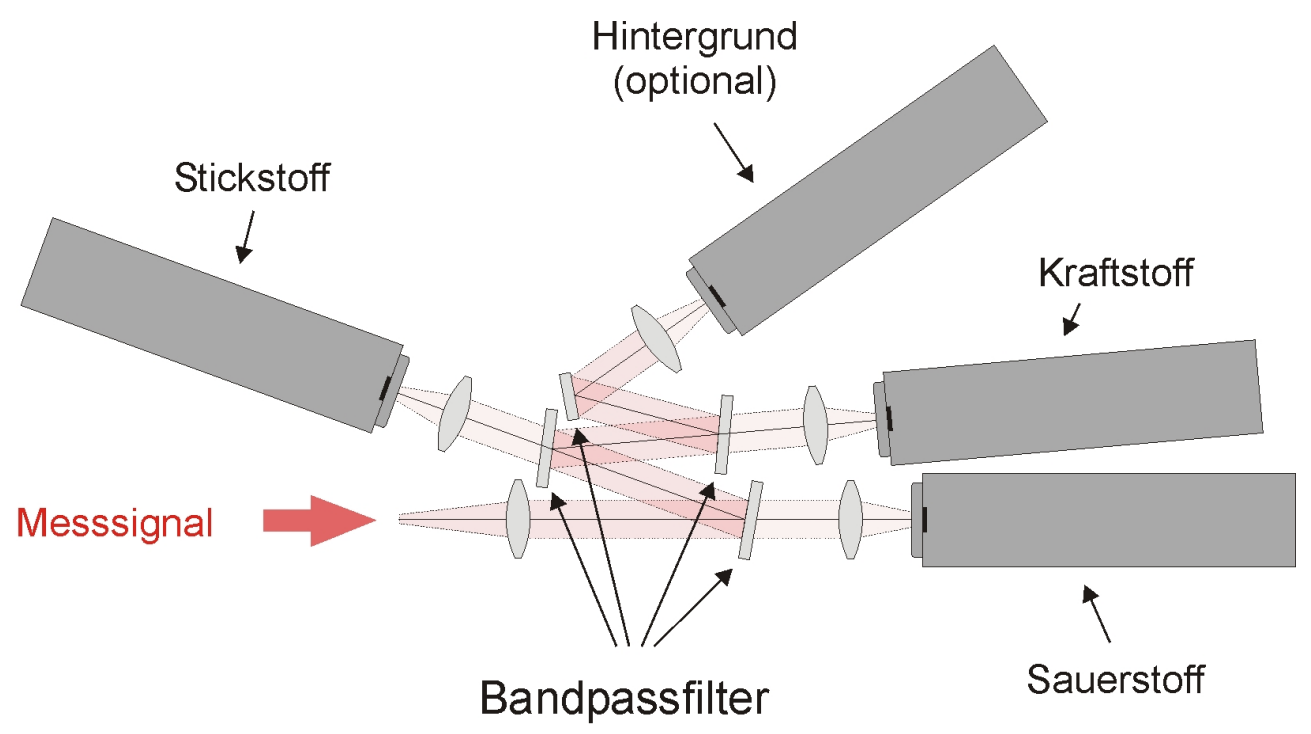

Abbildung 4.35.: Filter- und Detektoranordnung zur Separierung der einzelnen Raman-Signale

Es zeigt sich, dass durch die Bandpass-Filter eine gute spektrale Trennung erfolgt. Bei der Sauerstoffdetektion ist allerdings zu beachten, dass die Raman-Linie der CH-BiegeSchwingung mit einer Raman-Verschiebung von 1342,4 $\mathrm{cm}^{-1}$ [25] die Sauerstoff-RamanLinie bei $1556 \mathrm{~cm}^{-1}$ überlagert. Da die spektrale Bandbreite des Sauerstoff-Bandpassfilters nicht klein genug ist beide Linien spektral zu trennen, beinhaltet das gemessene RamanSignal immer Beiträge beider Spezies. Für eine korrekte Bestimmung des Lambda-Wertes ist es daher notwendig, das Verhältnis zum Stickstoff-Signal mitzubestimmen.

Eine optimal auf die jeweilige Raman-Spektrallinie angepasste Transmission wird unter Winkeln im Bereich von 10-13 ${ }^{\circ}$ zur Spiegelnormalen erzielt. Dadurch ergibt sich die Möglichkeit, die Filter zu kaskadieren und so die Raman-Signale der einzelnen Spezies nacheinander herauszufiltern. Dieses Prinzip wurde in einem Aufbau realisiert, der die simultane Detektion des Sauerstoff-, Stickstoff- und Kraftstoff-Raman-Signals erlaubt (Abbildung 4.35). Das einfallende Licht wird zunächst durch eine Linse kollimiert und auf den Sauerstoff-Raman-Filter geleitet. Das transmittierte Raman-Signal des Sauerstoffs wird auf einen Detektor abgebildet, während die langwelligeren Messsignalanteile auf den Stickstoff-Raman-Filter treffen. Anschließend wird das Kraftstoff-Signal mit Hilfe eines dritten Bandpass-Filters selektiert. Zusätzlich wäre die Aufnahme eines UntergrundSignals zur Korrektur der übrigen Messsignale mittels einer weiteren Filter/DetektorEinheit möglich.

Bei dieser Anordnung ist eine gute Kollimation des Lichtes notwendig, damit auf dem Weg zu und zwischen den einzelnen Filtern kein Signalverlust auf Grund einer zu starken Divergenz auftritt. Zudem würde sich der Transmissionsbereich der Filter bei anderen als durch den gewählten Kippwinkel auftretenden Einfallswinkeln des Lichtes auf die Filter verändern. 


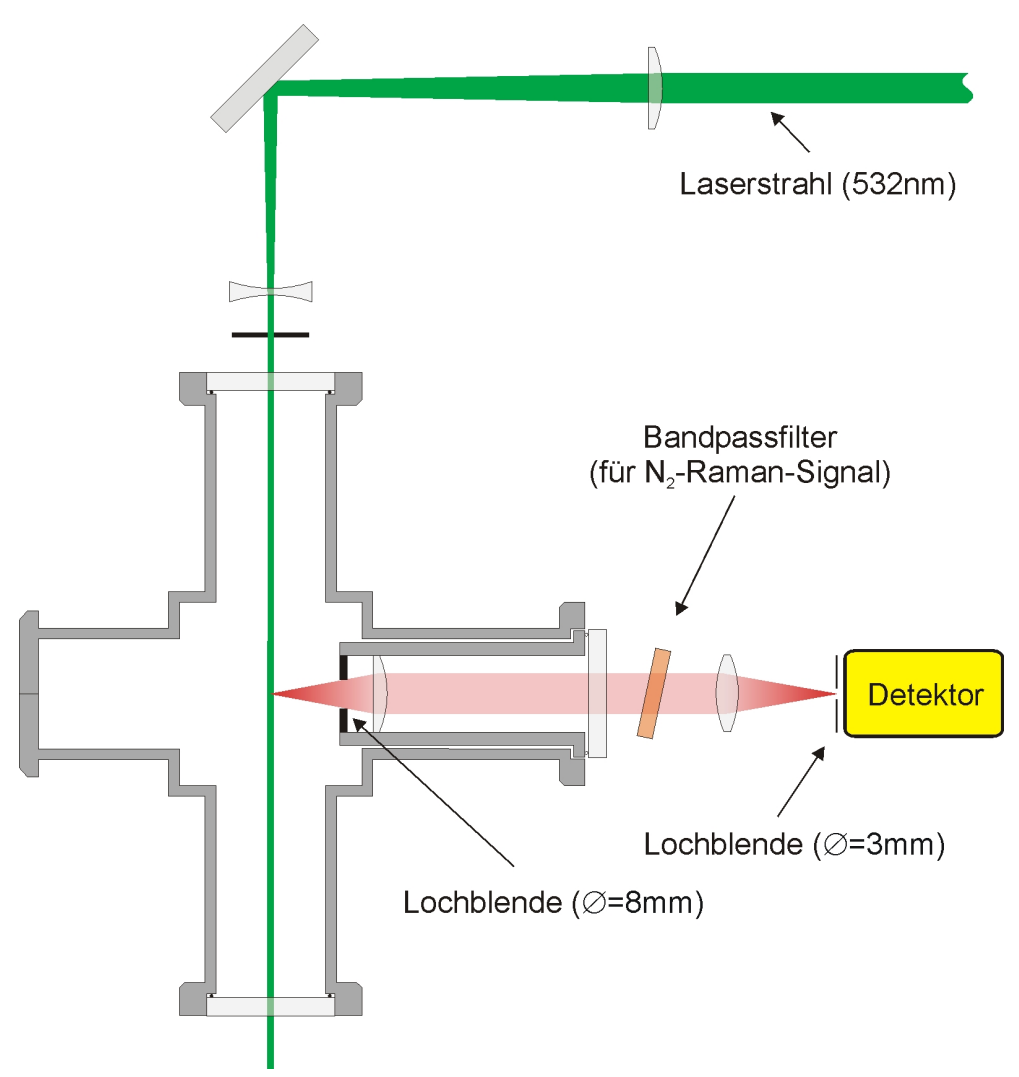

Abbildung 4.36.: Experimenteller Aufbau zur Charakterisierung verschiedener Detektoren

\subsubsection{Vergleich unterschiedlicher Detektoren}

Bei den bisherigen Raman-Untersuchungen wurde zur Detektion eine bildverstärkte CCDKamera verwendet. Die entstehenden zweidimensionalen Signalverteilungen geben einen Aufschluss über die Abbildungseigenschaften der Sammeloptiken und helfen diese zu optimieren. Allerdings ist die Arbeitsfrequenz der Kamera sehr niedrig, so dass eine Analyse der Lambda-Wert-Entwicklung innerhalb eines einzelnen Motorzyklus nicht möglich ist. Verzichtet man auf Ortsinformationen, ist die Verwendung schnellerer Detektoren möglich. Zwei Detektoren, ein Channel Multiplier Tube (CMT) der Firma Perkin Elmer (MH-720P) und eine Avalanche-Photodiode (APD) der Firma Hamamatsu (C4777-01-SPL-S53443), wurden hinsichtlich ihrer Linearität und ihres Rauschverhaltens untersucht und mit der Kamera verglichen.

Der experimentelle Aufbau zum Vergleich der Detektoren ist in Abbildung 4.36 skizziert. Um gleichzeitig zu untersuchen, ob prinzipiell Raman-Messungen mit einer gewählten Sensorkopfgeometrie eines Zündkerzenadapters möglich sind, wurde über ein Teleskop zunächst der Durchmesser des Nd:YAG-Laserstrahls von $8 \mathrm{~mm}$ auf $1,6 \mathrm{~mm}$ verkleinert und in eine Kreuzzelle eingekoppelt. Rechtwinklig zum Laserstrahl erfolgte die Detektion der 


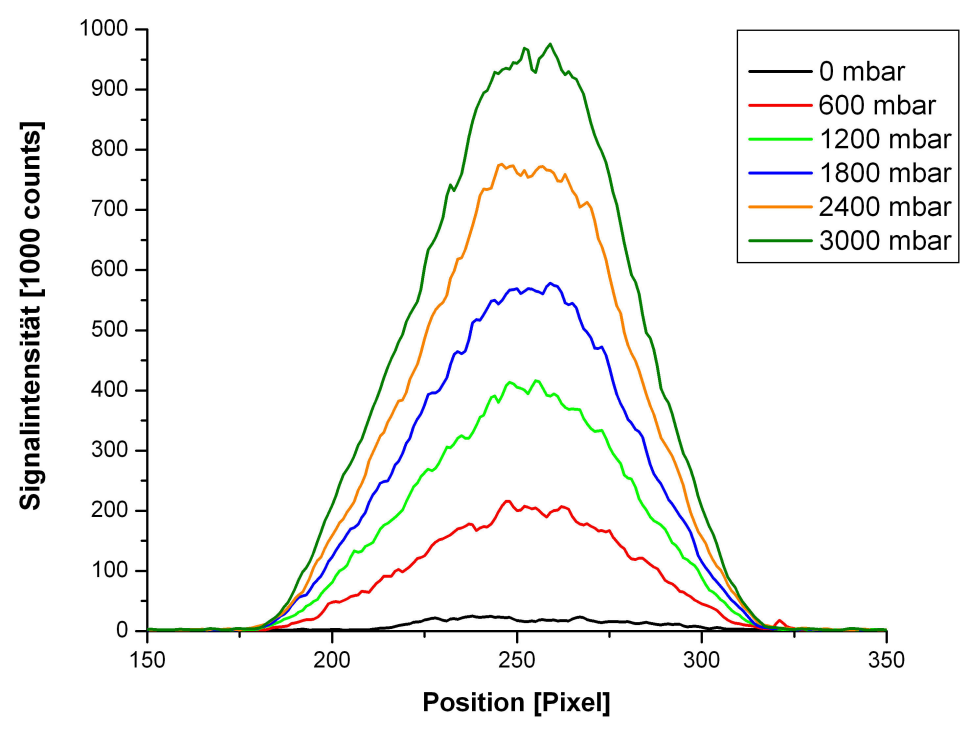

Abbildung 4.37.: Druckabhängigkeit der Raman-Signalverteilung bei der ICCD-Kamera (jeweils gemittelt über 250 Einzelschüsse, Testgas $=$ Stickstoff, Gain $=10.0$, Temperatur $=20^{\circ} \mathrm{C}$ )

Messsignale. Dabei wurde die f-Zahl der Sammeloptik mit Hilfe einer Lochblende von $8 \mathrm{~mm}$ Durchmesser, die im Abstand von $18 \mathrm{~mm}$ zum Messvolumen platziert wurde, auf 2, 25 festgelegt und damit auch an die Geometrie eines Sensorkopfes angepasst. Alle Untersuchungen erfolgten mit reinem Stickstoff als Testgas. Aus diesem Grund wurde aus dem Messsignal das Stickstoff-Raman-Signal über den verkippten LOT-Bandpass-Filter selektiert. Um sicher zu stellen, dass auf alle drei zu untersuchenden Detektoren (CMT, APD und ICCD-Kamera), trotz unterschiedlicher Eintrittsöffnungen, das gleiche Signal fiel, wurde eine weitere Lochblende mit 3mm Durchmesser vor den Detektoren positioniert. Die Pulsenergie des Lasers betrug $\sim 6 \mathrm{~mJ}$.

Zunächst wurden die Signaleigenschaften der bildverstärkten CCD-Kamera (ICCD) charakterisiert. Eine eindeutige Beziehung zwischen einer Messgröße, wie der Moleküldichte, und einem ermittelten Messwert ist Grundvoraussetzung für die Quantifizierbarkeit von Messergebnissen. Um dies zu untersuchen, wurden in der Zelle Drücke von 0-3000mbar in Schritten von 200mbar eingestellt und gemittelt über 250 Einzelschüsse die StickstoffRaman-Signale mit der CCD-Kamera aufgenommen. Das zweidimensionale Bild jeder einzelnen Aufnahme wurde dabei entlang einer Achse gemittelt und zu einem eindimensionalen Intensitätsprofil zusammengefasst. In Abbildung 4.37 ist die Raman-Signalverteilung in Abhängigkeit von verschiedenen Stickstoffdrücken dargestellt. Erfolgt eine örtliche Integration der Intensitätsprofile zu einem Gesamtsignal, zeigt sich eine sehr gute Linearität der aufgenommenen Intensität zum Druck (Abbildung 4.38). 


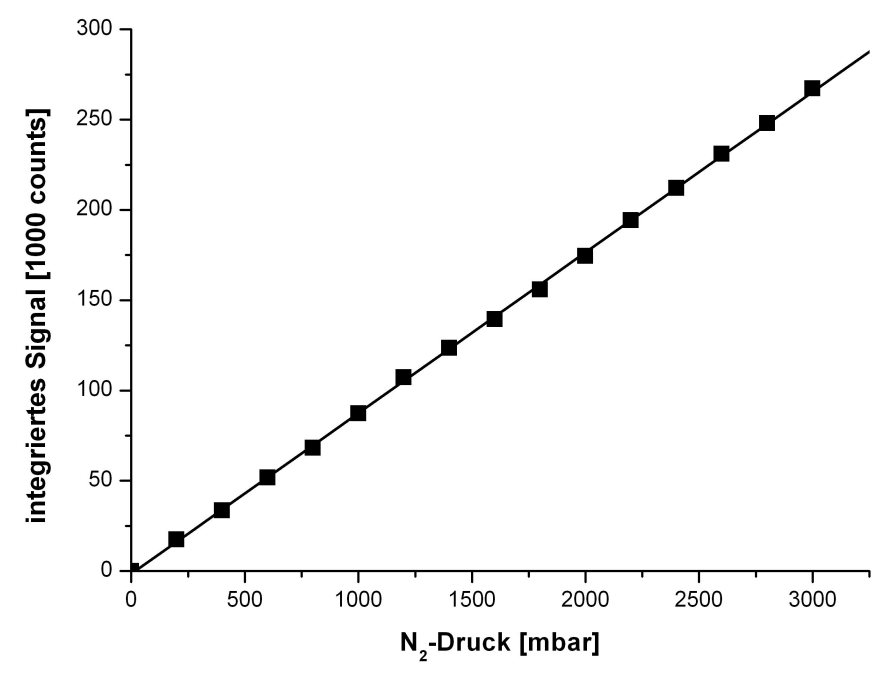

Abbildung 4.38.: Linearität der ICCD-Kamera-Signale zu verschiedenen Drücken (jeweils gemittelt über 250 Einzelschüsse, Testgas $=$ Stickstoff, Gain $=10.0$, Temperatur $=20^{\circ} \mathrm{C}$ )

Aus der Signalintensität der spontanen Raman-Streuung nach Gleichung 3.10 lässt sich theoretisch für die gewählte Sammeloptik die Anzahl von Photonen, die den Detektor erreichen, bestimmen. Der differenzielle Raman-Streuquerschnitt von Stickstoff beträgt $4,6 \cdot 10^{31} \mathrm{~cm}^{2} / \mathrm{sr}$ bei $532 \mathrm{~nm}$ [63]. Bei 3bar Druck, einem Detektionsvolumen von $5,12 \mathrm{~mm}^{3}$ und einer Temperatur von $20^{\circ} \mathrm{C}$ treffen 19682 Photonen auf die erste Linse (Streuverluste an Linsen und Fenstern werden nicht mitberücksichtigt). Die Quanteneffizienz des gesamten Detektionssystems $\eta_{c}$ setzt sich zusammen aus der CCD-Kamera mit 11\% und der Transmission des Bandpassfilters mit etwa 68\%. Daraus folgt, dass theoretisch 1472 Photoelektronen in der ICCD-Kamera erzeugt werden. Es ergeben sich aber aus dem tatsächlich gemessenen Signal nur 810 erzeugte Photoelektronen. Dies zeigt die bei dieser Kamera auftretenden elektronischen Verluste bei der Signalübertragung und Verarbeitung.

\begin{tabular}{|c|c|c|c|}
\hline Detektor & $\mathrm{QE}_{\text {Detektor }}[\%]$ & $\begin{array}{c}\text { Photoelektronen } \\
\text { (theoretisch) }\end{array}$ & $\begin{array}{c}\text { Photoelektronen } \\
\text { (gemessen) }\end{array}$ \\
\hline Kamera & 11 & 1472 & 810 \\
CMT & 11 & 1472 & 1410 \\
APD & 89 & 11912 & 6970 \\
\hline
\end{tabular}

Tabelle 4.2.: Berechnete und gemessene Anzahl von Photoelektronen am Detektor (3bar Druck, Temperatur $=20^{\circ} \mathrm{C}$ ) 
a)
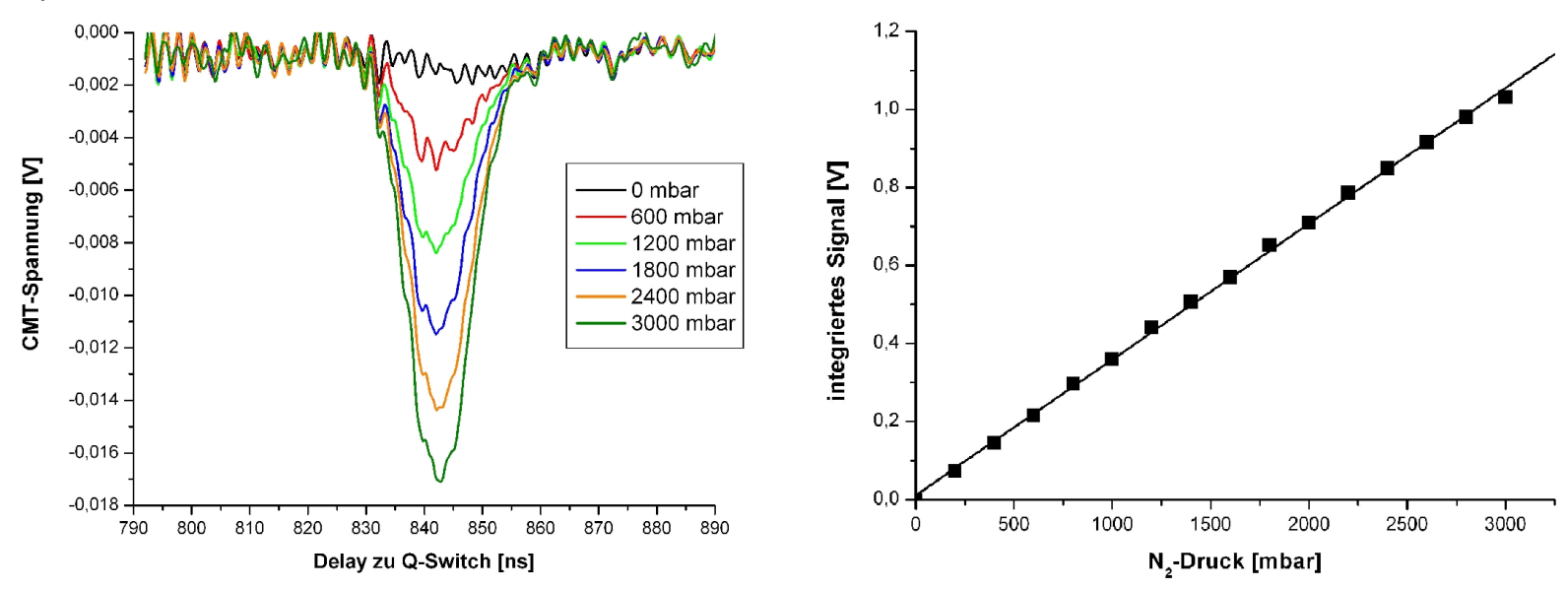

b)
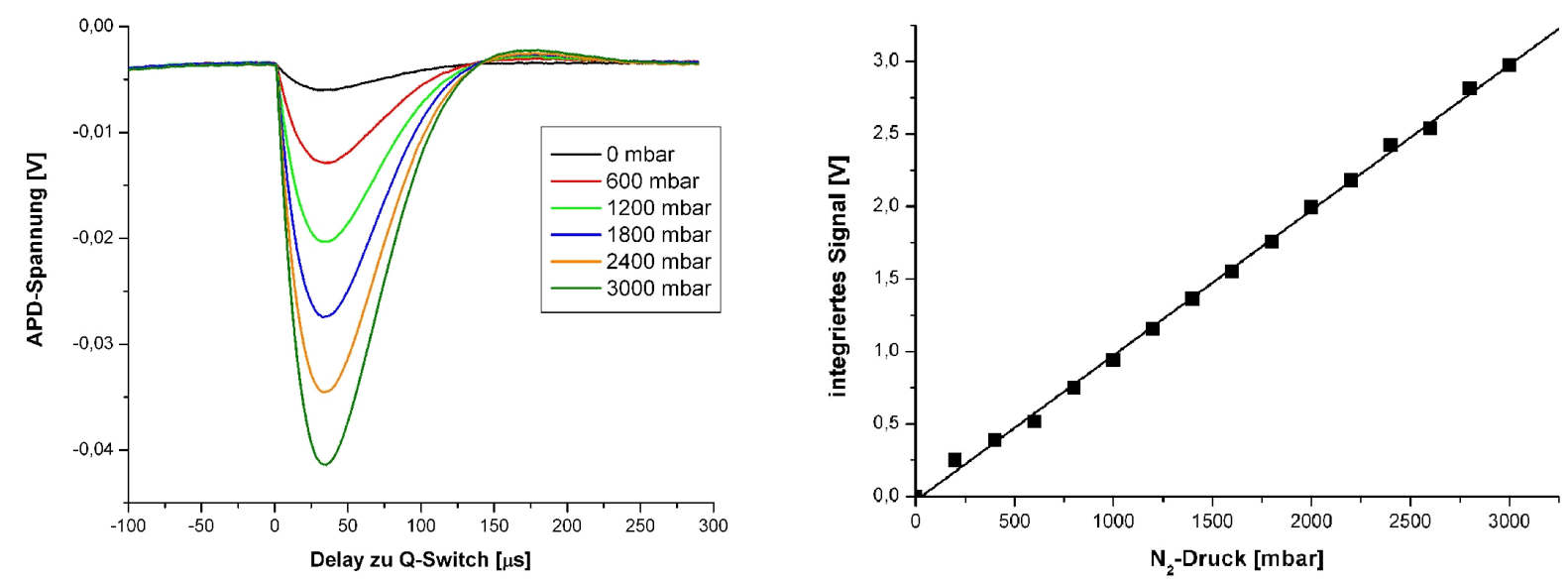

Abbildung 4.39.: Druckabhängigkeit des a) CMT-Signals und b) APD-Signals, jeweils zusammen mit der Abhängigkeit der integrierten Signale vom Druck (gemittelt über 250 Einzelschüssen, Testgas $=$ Stickstoff, Temperatur $=20^{\circ} \mathrm{C}$ )

Die gleichen Messungen wurden nun mit dem APD und dem CMT durchgeführt, um diese zu charakterisieren. Beide zeigen das gleiche lineare Verhalten wie die Kamera (siehe Abbildung 4.39). Die eingesetzte Channel Multiplier Tube (CMT) reagierte dabei empfindlich auf äußere elektrische Felder. Die in Abbildung 4.39a den CMT-Signalen überlagerte Schwingung resultierte von der beim Q-Switch das Lasers angesteuerten Pockelszelle. Diese Schwingung war daher bei allen Messungen identisch und trägt somit nicht zum Rauschen bei. Die Verstärkung der erzeugten Photoelektronen ist beim CMT durch die Wahl der Verstärkungsspannung im Multiplier einstellbar. Allerdings bestand bei großer Spannung keine Linearität mehr zwischen CMT-Signal und Druck, da der erzeugte Anodenstrom im gepulsten Betrieb auf Grund der hohen Photonendichte zu groß war. Die 
schon am Anfang des Pulses im Multiplier entstehende große Elektronenwolke kann durch die lange Regenerationszeit des CMT von etwa $3 \mu$ s nicht abgeführt werden, so dass das Licht am Ende des Laserpulses nicht mehr detektiert wird. Bei der Verwendung von CWLasern tritt dieses Problem aber nicht auf.

Für die beiden Detektoren wurden ebenfalls die bei 3bar Stickstoff-Druck in den Detektoren theoretisch erzeugten Photoelektronen berechnet (siehe Tabelle 4.2). Die Messung zeigte, dass durch die höhere Quanteneffizienz von etwa $89 \%$ unter gleicher Beleuchtungsstärke bei der APD fast neun mal mehr Photoelektronen erzeugt werden, wie bei der Kamera oder dem Channel-Multiplier-Tube (CMT). Die Verluste in diesem Detektor sind allerdings im Verhältnis deutlich größer.

Bei den bisherigen Messungen zum Detektorvergleich wurden die Messwerte über viele Einzelschüsse gemittelt. Die hohe Geschwindigkeit der Gemischbildungsprozesse und die zyklischen Schwankungen machen aber Einzelmessungen mit einer hohen zeitlichen Auflösung notwendig. Die Genauigkeit dieser Messungen hängt vom Signal/RauschVerhältnis ab. Die Photoelektronenstatistik liefert dabei eine entscheidende Aussage über den Messfehler $\sigma$ eines optisch-elektrischen Detektorsystems. Das Rauschen der Einzelphotoelektronen (shot noise) definiert die untere Grenze über die Anzahl der gemessenen Photoelektronen $N$.

$$
\sigma_{P e l}=\frac{1}{\sqrt{N}}
$$

Damit würde die Avalanche-Photo-Diode (APD) die besten Voraussetzungen besitzen. Mit einer Quanteneffizienz von nahezu 90\% werden bei diesem Detektor unter gleicher Beleuchtungsstärke fast neunmal so viele Photoelektronen erzeugt, wie bei den anderen beiden Detektionssystemen. Um dies zu klären, wurden nun statistische Untersuchungen durchgeführt, bei denen zu jedem Messpunkt eine Anzahl von Einzelmessungen erfolgte. Bei der Kamera waren dies 100 und bei der APD 1000 Einzelmessungen. Der CMT wurde bei diesen Untersuchungen nicht eingesetzt, da keine Elektronik zur Verfügung stand, die die Aufnahme einer Vielzahl von Einzelmessungen ermöglicht hätte. Bei der Kamera konnten die Einzelmessungen mit Hilfe der zugehörigen Software aufgenommen werden, während die zeitliche Integration der APD-Signale über einen Boxcar-Integrator erfolgte. Dessen Ausgangsspannung wiederum wurde mit einem Analog/Digital-Konverter in einen PC eingelesen. Die Ergebnisse dieser Untersuchungen sind in Abbildung 4.40 dargestellt. Dabei markieren die Fehlerbalken den 2s-Bereich der Einzelwertstatistik. Dies bedeutet das über 95\% der Einzelmessungen innerhalb des angezeigten Intervalls liegen. Für den angegebenen relativen Messfehler gilt das ebenso. Zum Vergleich sind zusätzlich noch die sich alleine aus der Photoelektronenstatistik ergebenden Fehlerwerte dargestellt. Bei beiden Untersuchungen liegt der effektive Gesamtmessfehler $\sigma$ deutlich oberhalb des Fehlers $\sigma_{P e l}$. Hierbei muss allerdings noch berücksichtigt werden, dass die Puls-zu-PulsSchwankungen des Lasers von 5\% darauf einen Einfluss haben. Insgesamt folgt aus diesen statistischen Untersuchungen, dass sich für einen gepulsten Laserbetrieb die AvalanchePhoto-Diode am besten eignet. Neben ihrer hohen Quanteneffizienz liegt ihr Fehler bei Einzelmessungen bereits ab einem Stickstoffdruck von 1bar mit einer Wahrscheinlichkeit 
a)

\section{bildverstärkte CCD-Kamera}

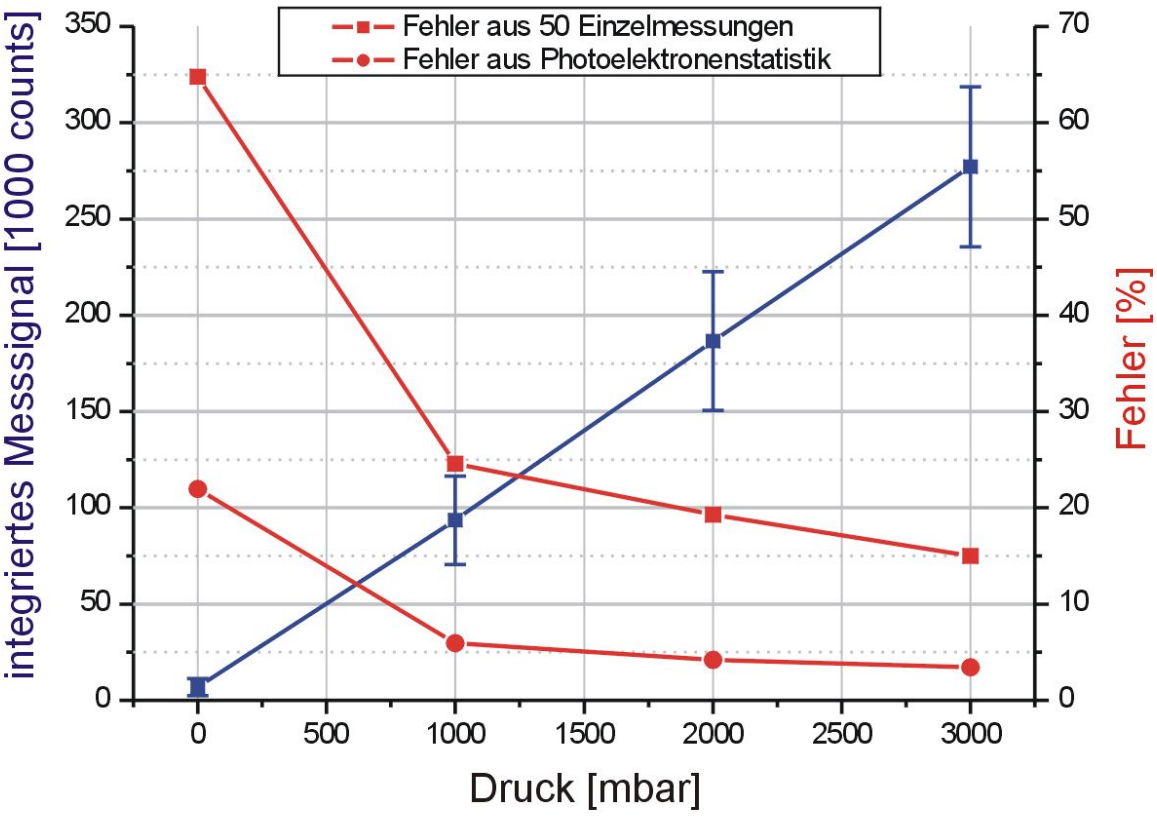

b)

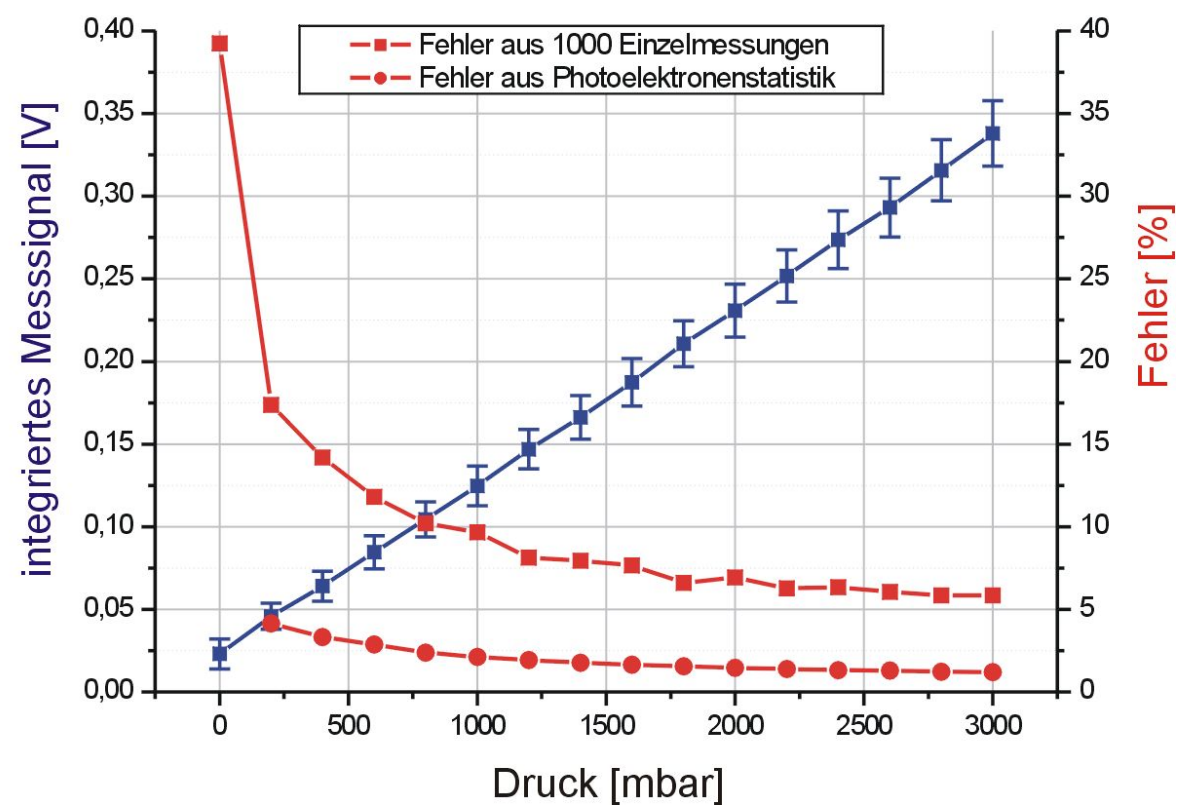

Abbildung 4.40.: Vergleich der Ungenauigkeit von Einzelmessungen bei verschiedenen $\mathrm{N}_{2}$-Drücken, wobei eine Messsicherheit von $\mathrm{P}>95 \%$ vorausgesetzt wurde (2s-Intervall) a) bildverstärkte CCD-Kamera b) Avalanche Photo Diode 
von über $95 \%$ unterhalb von $10 \%$.

Anders verhält sich das bei Raman-Messungen mit einem kontinuierlichen Laser. In dem Fall werden auf Grund der geringen Photonendichte auch bei hohen Moleküldichten nur sehr kleine Raman-Streulichtsignale auftreten. Bei der APD gehen diese kleinen Signale im hohen Grundrauschen des Detektors unter. Dagegen ist der CMT für Einzelphotonenereignisse ausgelegt und ermöglicht so die Detektion von extrem schwachen Lichtsignalen. 


\subsubsection{Erste Untersuchungen von Gemischzusammensetzungen}

Mit Hilfe des in Abschnitt 4.5.2 beschriebenen Detektionssystems wurden nun erste Messungen an einem Luft/Kraftstoffgemisch vorgenommen. Dabei wurde das System mit drei zur Verfügung stehenden Channel-Multiplier-Tubes (CMT) ausgerüstet. Die gleichzeitige Aufzeichnung der Signale dieser Detektoren erfolgte mittels eines Oszilloskops. Der experimentelle Aufbau der Laserstrahlführung und der Sammeloptik des Streulichtes entsprach dem in Abbildung 4.36 dargestellten Aufbaus. An Stelle des Detektors wurde im experimentellen Aufbau eine Glasfaser (Durchmesser 1,5mm) platziert, um das in der Druckzelle erzeugte Raman-Licht zum Detektionssystem zu übertragen. Ein in einem Vormischbehälter erzeugtes Luft/Kraftstoffgemisch ermöglichte es, die Druckzelle mit verschiedenen Drücken, aber gleichbleibendem Verhältnis der Spezies zu befüllen.

Aus 256 Einzelschüssen gemittelt wurden nun für Stickstoff, Sauerstoff und Kraftstoff die Raman-Signale aufgezeichnet und dann über den zeitlichen Verlauf integriert. In Abbildung 4.41 ist der Verlauf der Signalintensitäten in Abhängigkeit vom Gesamtdruck dargestellt. Dabei zeigt sich ein nahezu linearer Zusammenhang der detektierten Raman-Streulichtintensitäten zur Konzentration. Das Sauerstoffsignal allerdings ist durch die mangelnde spektrale Separation vom Kraftstoffsignal der CH-Biege-Schwingung überlagert (vergl. Abschnitt 4.5.2). Auf einem Detektor werden also die Signale zweier Spezies gleichzeitig gemessen. Zusätzlich führen bei allen drei Signaldetektionen die hohen Photonendichten durch den gepulsten Laser und dem daraus resultierenden großen Anodenstrom zusammen mit der langen Regenerationszeit des CMT insbesondere bei höheren Drücken zu einer Abweichung von einem linearen Zusammenhang. Dies ließe sich aber bei gepulster Laseranregung durch die Verwendung von Avalanche-Photo-Dioden beheben.

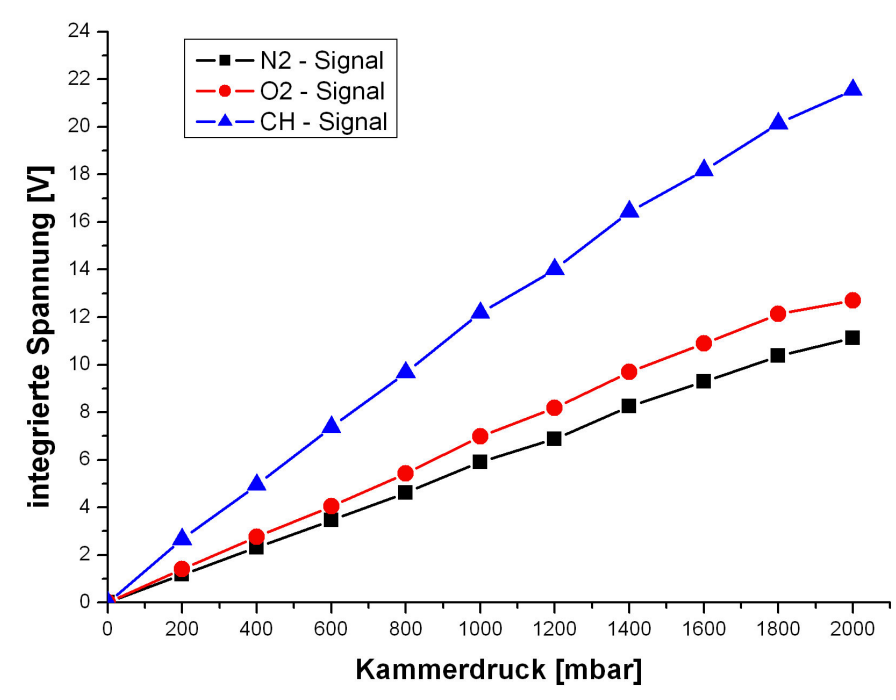

Abbildung 4.41.: Erste Messungen an einem Luft/Kraftstoffgemisch in der Druckzelle 


\subsection{Zusammenfassung und Ausblick}

In diesem Kapitel wurde eine Methode vorgestellt, mit der die Bestimmung des LambdaWerts im Brennraum mit Hilfe spontaner Raman-Streuung möglich ist. Um reale Bedingungen der Gemischbildungsprozesse untersuchen zu können, erfolgten alle Messungen mit Euro Super-Benzin. Der große Anteil an aromatischen Kohlenwasserstoffverbindungen, wie z.B. Benzol, machte die Verwendung eines Nd:YAG-Lasers als Lichtquelle im sichtbaren Wellenlängenbereich bei 532nm notwendig. In Voruntersuchungen an Kraftstoffsprays in einer Druckkammer stellte sich heraus, dass bei sichtbarer Laseranregung in tröpfchenbeladenen Strömungen nichtlineare stimulierte Raman-Streuung auftritt. Um Streulichtemissionen aus der Flüssigphase von Realkraftstoffsprays zu unterdrücken und deren Einfluss auf den gemessenen Lambda-Wert zu minimieren, wurde dem Kraftstoff ein Absorberfarbstoff beigemengt. Darüber hinaus wurden, wie in Kapitel 4.1 gezeigt, die Laserpulse zeitlich gestreckt und das Strahlprofil homogenisiert, um zerstörungsfrei in einem Transparentmotor mit hohen Laserleistungen messen zu können. So war es erstmals möglich an einem Versuchsmotor eines Auftraggebers aus der Automobilindustrie Messungen mit Realkraftstoff durchzuführen. Ziel der Untersuchungen war es, eine 1dimensional ortsaufgelöste Lambda-Wert-Verteilung mittels spontaner Raman-Streuung am Rand des eingespritzten Kraftstoffsprays zu ermitteln, um so die optimale Position für eine Zündkerze in einem strahlgeführten Brennverfahren zu finden. Um eine größere Aussagekraft zu erzielen, wurden die Raman-Messungen mit einer flächigen Fluoreszenzuntersuchung kombiniert. Die Variation von motorischen Bedingungen und die Charakterisierung des Sprayverhaltens unterschiedlicher Einspritzventile zeigte die Einsetzbarkeit des neuen Messkonzepts an benzindirekteinspritzenden Ottomotoren.

Da Serienmotoren nur über wenige optisch nutzbare Zugänge in den Brennraum verfügen, sollte ein Sensor entwickelt werden, der an Stelle der Zündkerze eingeschraubt wird und über Glasfasern mittels spontaner Raman-Streuung den Lambda-Wert am Ort der Zündkerze ermittelt. Voruntersuchungen gaben Aufschluss über die Probleme eines faseroptischen Sensorsystems bei hohen Laserleistungen. Die Laseranregung erfolgte wie bei den vorherigen Messungen mit dem Nd:YAG-Laser. Dieses Sensorsystem wurde ebenfalls am Versuchsmotor eingesetzt. Es zeigte sich, dass mit der gewählten Sensorgeometrie gemittelte Messungen möglich waren. Auf Grund des geringen Raman-Signals konnten aber keine Einzelaufnahmen ausgewertet und zyklische Schwankungen ermittelt werden.

Um eine zyklusaufgelöste Bestimmung der Lambda-Wert-Entwicklung zu ermöglichen, wurde ein neues Detektionskonzept entwickelt. Dabei erfolgten keine ortsaufgelösten Messungen, sondern der Lambda-Wert wurde lokal am Ort der Zündkerze bestimmt. Grundlegende Untersuchungen ergaben, dass eine spektrale Separation des Raman-Streulichts der relevanten Molekülspezies Sauerstoff, Stickstoff und der Kohlenwasserstoffe über eine kaskadierte Filteranordnung mit mehreren Photomultiplieren eine optimale Detektion sehr kleiner Messsignale ermöglicht. In ersten Messungen konnte die Einsatzfähigkeit dieses Systems unter Beweis gestellt werden. 
Insgesamt ist dieses vorgestellte Detektionssystem zur Lambda-Wert-Bestimmung mittels spontaner Raman-Streuung zwar ein technisch aufwendiges, dafür aber sehr genaues Messprinzip. Derzeit laufen erste Untersuchungen an einem Ottomotor. In Zukunft soll eine leicht geänderte Sensorkopfgeometrie auch die Möglichkeit der Zündung des Kraftstoffgemischs beinhalten. 


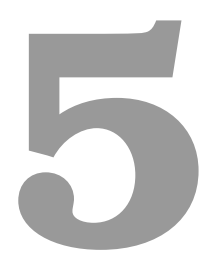

\section{Gemischbildungsanalyse mittels Absorptionsspektroskopie}

Für die Analyse von Verbrennungsprozessen in Motoren und deren Optimierung ist neben der zeitlichen Entwicklung des $\lambda$-Wertes in einem Zyklus auch die Kenntnis der Kraftstoffkonzentration und die Konzentrationen der durch die Verbrennung im vorhergehenden Zyklus erzeugten Restgase, wie z.B. $\mathrm{CO}_{2}, \mathrm{H}_{2} \mathrm{O}$, Stickoxide oder unverbrannte Kohlenwasserstoffverbindungen wichtig. Eine Möglichkeit der direkten Bestimmung des Luft/Kraftstoffverhältnisses bietet die in Kapitel 4 vorgestellte Raman-spektroskopische Gemischanalyse. Im Gegensatz zu dieser Messtechnik sind allerdings die Wirkungsquerschnitte bei der Absorptionsspektroskopie, beispielsweise im infraroten Spektralbereich, um etwa 10 Größenordnungen höher [52]. Dadurch ist eine Bestimmung sehr viel geringerer Molekülkonzentrationen möglich als bei der Raman-Spektroskopie. Die Absorptionsspektroskopie ist eine sehr einfache Messmethode, die es auch erlaubt mit nur sehr kleinen optischen Zugängen, wie der Zündkerzenbohrung, Einblicke in die Prozesse im Motor zu gewinnen. Allerdings ist sie ein integrales Messverfahren über die Absorptionsstrecke hinweg und damit nicht ortsauflösend.

In diesem Kapitel soll zunächst eine Messtechnik vorgestellt werden, bei der mit Hilfe der Absorption im infraroten Spektralbereich die Kraftstoffkonzentration am Ort der Zündkerze bestimmt werden kann. Über eine kurze Absorptionsstrecke in einem Zündkerzenadapter ist es damit möglich, die Konzentration von Realkraftstoff kurbelwinkelaufgelöst an einem Serienmotor zu messen. Unter bestimmten Voraussetzungen kann mit 
diesem System der Lambda-Wert näherungsweise bestimmt werden.

Desweiteren wird eine Messmethode und deren experimenteller Aufbau zur Bestimmung der Sauerstoffkonzentration beschrieben. Dabei wird die Absorption des Lichtes eines Vertical Cavity Surface-Emitting Lasers (VCSEL-Laser) um 760nm durch die $\mathrm{O}_{2^{-}}$ Moleküle ausgenutzt. Durch die Schmalbandigkeit des Lasers können einzelne Rotationslinien spektral aufgelöst werden. Daraus ergeben sich verschiedene Möglichkeiten die Temperatur des Gases zu bestimmen. Die Einsetzbarkeit und Grenzen dieser Messtechnik unter innermotorischen Bedingungen wird diskutiert.

\subsection{Kraftstoffdichtebestimmung mittels Absorptionsspektroskopie im mittleren Infrarot}

Im mittleren infraroten Wellenlängenbereich $2 \mu \mathrm{m}<\lambda<20 \mu \mathrm{m}$ besitzen viele Molekülspezien starke Absorptionsbanden auf Grund von Energieübergängen zwischen Vibrationsniveaus. Auch die an innermotorischen Verbrennungsprozessen beteiligten Moleküle absorbieren infrarotes Licht, beispielsweise $\mathrm{CO}_{2}$ bei $4,3 \mu \mathrm{m}$ und $15 \mu \mathrm{m}$, CO bei etwa $4,7 \mu \mathrm{m}$ und NO bei $5,3 \mu \mathrm{m}$. Wasser besitzt sehr breite Absorptionsbanden zwischen $2,5 \mu \mathrm{m}-3 \mu \mathrm{m}$ und $5 \mu \mathrm{m}-8 \mu \mathrm{m}$.

Die Entwicklung von im infraroten Wellenlängenbereich transparenten Lichtwellenleitern machte in diesem Spektralbereich arbeitende faseroptische Sensoren erst möglich. Da die in der Telekommunikation eingesetzten Glasfasern kein Licht oberhalb einer Wellenlänge von etwa $2 \mu \mathrm{m}$ transmittieren, werden daher meistens Saphir- oder Chalkogenid-Fasern eingesetzt. Letztere besitzen zwar eine geringe Dämpfung $(<4 \mathrm{~dB} / \mathrm{m})$ im Spektralbereich zwischen etwa $2,5 \mu \mathrm{m}$ bis $12,5 \mu \mathrm{m}$, sind aber sehr spröde und nur bis zu Temperaturen um $300^{\circ} \mathrm{C}$ verwendbar. Saphirfasern besitzen dagegen eine sehr hohe Schmelztemperatur von über $2000^{\circ} \mathrm{C}$ und können daher auch unter Verbrennungstemperaturen eingesetzt werden. Allerdings sind sie nur bis etwa $3,7 \mu \mathrm{m}$ transparent, was die Anzahl der detektierbaren Molekülspezies stark einschränkt.

\subsubsection{Experimenteller Aufbau}

Für die Bestimmung der Kraftstoffdichte mit Hilfe der Absorptionsspektroskopie wird insbesondere die Absorption von Licht durch Vibrationsübergänge im Spektralbereich um 3,4 $\mu \mathrm{m}$ ausgenutzt (siehe Abschnitt 3.3). Das aus der Grundschwingung der C-H-

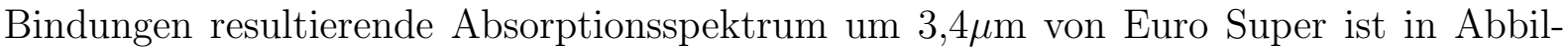
dung 5.2 dargestellt, welches mit Hilfe eines FT-IR-Spektrometers der Firma Brucker aufgenommen wurde. Da der Kraftstoff aus vielen verschiedenen Kohlenwasserstoffverbindungen besteht, bei denen jeweils durch unterschiedliche intramolekulare Kräfte die 


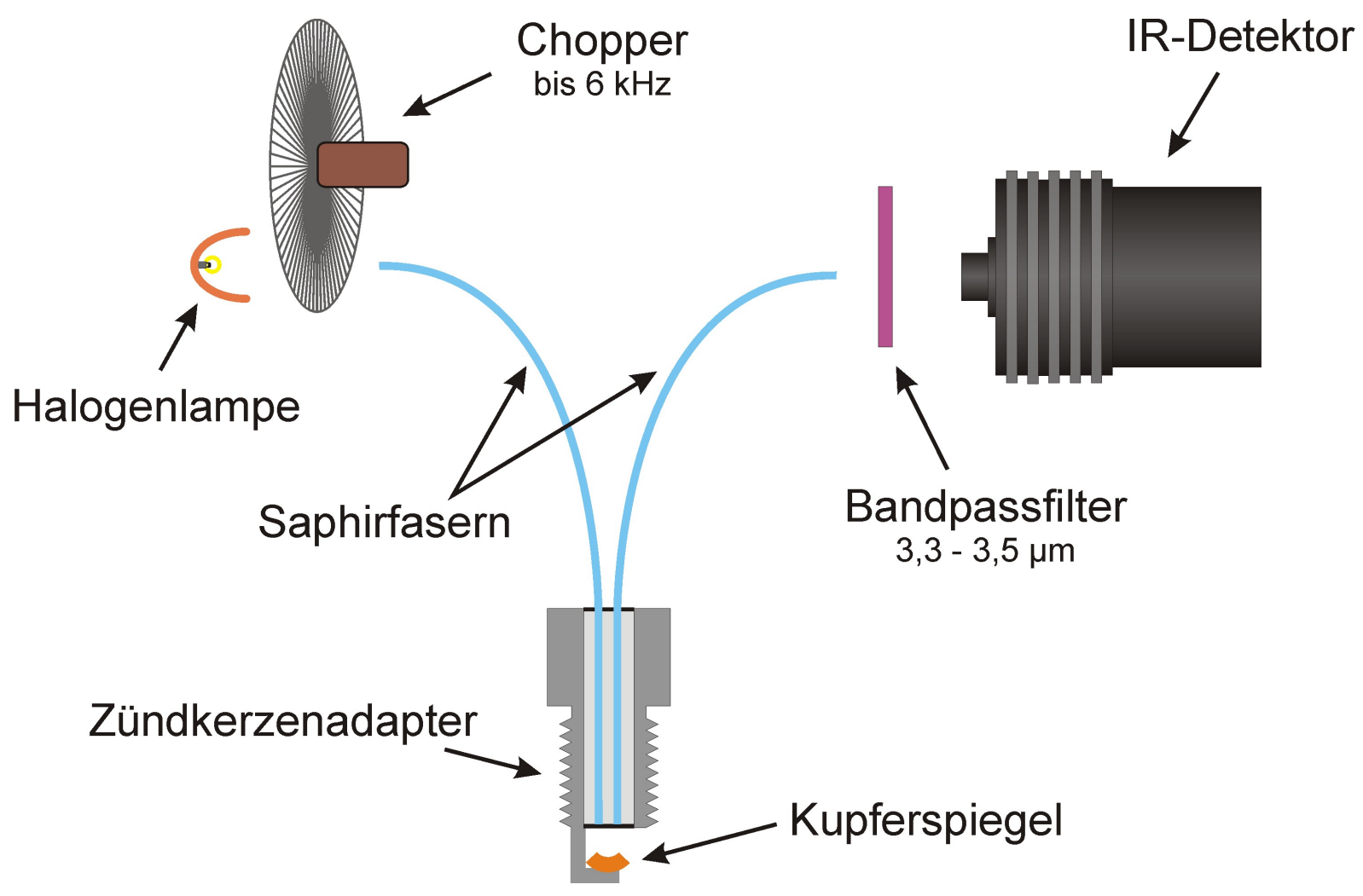

Abbildung 5.1.: Experimenteller Aufbau des Messsystems zur Bestimmung der Kraftstoffdichte im Motor

spektrale Position der Absorptionslinien variiert, überlagern sich die einzelnen Rotationslinien zu einer breiten Absorptionsbande.

Koenig und Hall [33, 34] haben eine Messmethode vorgestellt, bei der als Lichtquelle eine Halogenlampe verwendet wird. Ein ähnliches Messsystem wurde auch im Rahmen dieser Arbeit für motorische Untersuchungen realisiert. Der Aufbau dieses Systems ist in Abbildung 5.1 skizziert. Dabei wird das Licht einer Halogenlampe mit Hilfe eines elliptischen, goldbeschichteten Reflektors auf das Ende einer 1,5m langen Saphirfaser mit $425 \mu \mathrm{m}$ Durchmesser (Saphicon Inc.) fokussiert und in einen Zündkerzenadapter geleitet. Dieser Adapter lässt sich anstelle einer Zündkerze in einen Ottomotor einschrauben. Das aus der Faser austretende Licht wird nach einer kurzen Absorptionsstrecke im Brennraum durch einen goldbeschichteten konkaven Kupferspiegel (Radius 8mm) in eine zweite Saphirfaser fokussiert. Die Absorptionsstrecke beträgt damit 16mm. Da die Fasern durch ein $1 \mathrm{~mm}$ dickes Saphirfenster vor Verunreinigungen und Schäden geschützt werden, reduzierte sich der eigentliche Absorptionsweg auf $14 \mathrm{~mm}$. Als Detektor wird ein Peltier-gekühlter HgCdTe-Detektor (Vigo - PDI-2TE-4) verwendet. Der vor dem Detektor platzierte Bandpassfilter mit einer Zentralwellenlänge von $3,42 \mu \mathrm{m}$ und einer Halbwertsbreite von etwa 150nm lässt gerade nur den Wellenlängenbereich der CH-Absorptionsbande durch. Befin- 


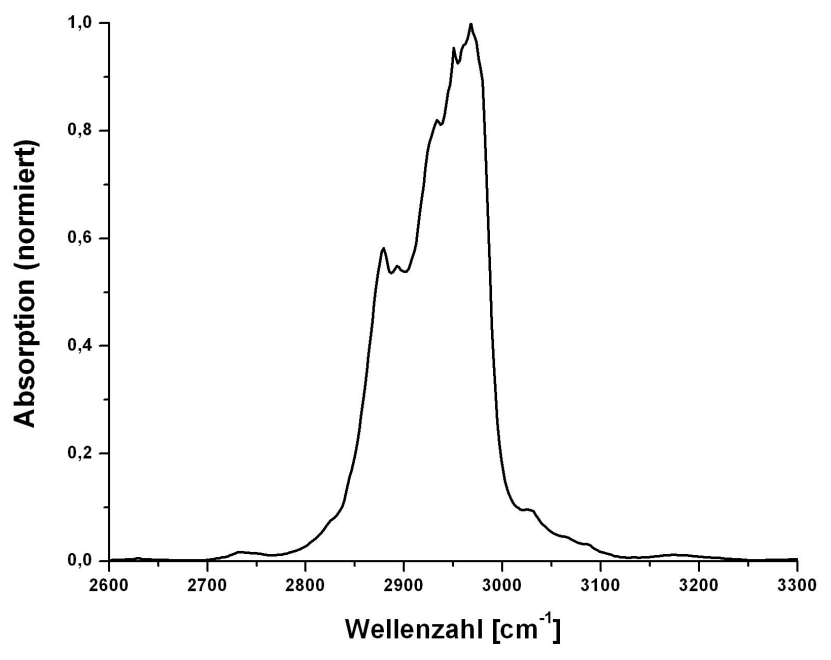

Abbildung 5.2.: Absorptionsspektrum von Euro Super um 3,4 $4 \mathrm{~m}$

det sich Kraftstoff in der Absorptionsstrecke zwischen den Saphirfasern und dem Kupferspiegel im Zündkerzenadapter, so führt dies zu einem Absinken der detektierten Lichtintensität. Wenn keine Beleuchtung durch die Halogenlampe erfolgt, lässt sich eine mögliche infrarote Untergrundstrahlung ermitteln, die insbesondere beim Einsatz im Brennraum durch die Kompression und die dabei auftretende Erhitzung des Kraftstoffgemischs zu erwarten ist. Genau diese beiden Zustände werden durch den Einsatz eines mechanischen Choppers erzeugt.

Mit Hilfe eines Spannungsverstärkers (Femto - DLPVA-100) ist es möglich, das Signal/Rausch-Verhältnis des Detektorsignals zu verbessern, da der Verstärker zusätzlich mit einem Tiefpassfilter bis $100 \mathrm{kHz}$ ausgerüstet ist. Ein Sample\&Hold-Board und ein A/D-Wandler (PlugIn - CIO1M) ermöglichen die Datenaufnahme mit einem PC.

\subsubsection{Messungen unter motorischen Bedingungen}

Erste Untersuchungen an einem geschleppt betriebenen Ottomotor sollten nun die Einsatzfähigkeit des Detektionssystems unter motorischen Bedingungen zeigen. An diesem Motor erfolgte die Einspritzung des Kraftstoffs in das Saugrohr, so dass schon im Ansaugtakt ein Luft/Kraftstoffgemisch in den Brennraum gelangte und sich dort homogen vermischen konnte. Die Frequenz des Choppers betrug bei diesen Messungen $5 \mathrm{kHz}$ und die Leistung der Halogenlampe 75W. In Abbildung 5.3 sind das IR-Detektorsignal und der gleichzeitig aufgezeichnete relative Zylinderdruck eines kompletten ungefeuerten Motorzyklus bei 1050 U/min dargestellt, bei dem Euro Super eingespritzt wurde. Die Druckkurve verdeutlicht hierbei den zeitlichen Verlauf der Kompression im Motorzyklus. In dieser Darstellung erscheint das schnell modulierte IR-Detektionssignal durch die geringe Zeitauflösung als 
breites Band.

In der Darstellung ist zu Beginn der Einlassphase ein leichtes Absinken der IRLichtintensität zu sehen. Dies zeigt, dass zunächst eintretender Kraftstoff am Ort der Zündkerze vorbei strömt und dann durch nachströmende Luft nach unten in den Brennraum verdrängt wird. Mit steigendem Druck während der Kompressionsphase sinkt das Signal dann wieder stark, d.h. die Kraftstoffkonzentration in der Absorptionsstrecke an der Zündkerze nimmt zu. Gleichzeitig ist zu beobachten, dass das Hintergrundsignal ebenfalls steigt. Es wird also Infrarotstrahlung detektiert, obwohl keine von der Halogenlampe eingestrahlt wird. Erfolgte keine Einspritzung, so dass nur eine Kompression der angesaugten Luft stattfindet, konnte kein Hintergrundsignal detektiert werden. Dies lässt darauf schließen, dass die vom Sensor detektierte Hintergrundstrahlung allein vom in Folge der Kompression aufgeheizten Kraftstoff resultiert und nicht von Brennraumoberflächen, wie z.B. dem sich nach oben bewegenden Kolben oder der Spiegeloberfläche des Sensor verursacht wird. Während der Auslassphase wird das Luft/Kraftstoffgemisch durch die geöffneten Auslassventile gepresst, was sowohl zu einem erneuten leichten Anstieg des Druckes als auch der Absorption führt.

Zur Verdeutlichung des durch den Chopper mit $5 \mathrm{kHz}$ modulierten Infrarotsignals ist in Abbildung 5.4 ein Ausschnitt aus dem Motorzyklus kurz vor dem oberen Totpunkt (OT) dargestellt. Mit Hilfe eines selbstentwickelten Software-Programms können wie bei einem Hardware-Boxcar-Averager Zeitintervalle in einem Signalzyklus gewählt werden, in denen dann mehrere Datenpunkte zusammengefasst und gemittelt werden. Dadurch ist es möglich das Gesamt- und das Hintergrundsignal innerhalb einer Modulationsperiode zu bestimmen. Die Differenz zwischen diesen beiden Signalen ist das reine Absorptionssignal, welches normiert mit der Anfangsintensität die Transmission ergibt. Die Zeitauflösung ist abhängig von der Chopperfrequenz und entspricht bei $5 \mathrm{kHz}$ einer Auflösung von 0,2ms oder auf motorische Anwendungen bezogen bei 1000U/min einer Zeitauflösung von $1,2^{\circ} \mathrm{KW}$.

Das Ergebnis einer solchen Analyse ist in Abbildung 5.5 gezeigt und wurde aus dem in Abbildung 5.3 dargestellten Rohsignal gewonnen. Das darin beschriebene Verhalten des Detektionssignals zeigt sich nun wesentlich deutlicher.

Mit diesem Messsystem ist es also möglich, die Absorption durch den Kraftstoff am Ort der Zündkerze kurbelwinkelaufgelöst in einem kompletten Motorzyklus zu bestimmen. Für die absolute Bestimmung der Kraftstoffkonzentration ist eine Kalibrierung mit bekannten Gemischzusammensetzungen notwendig. 


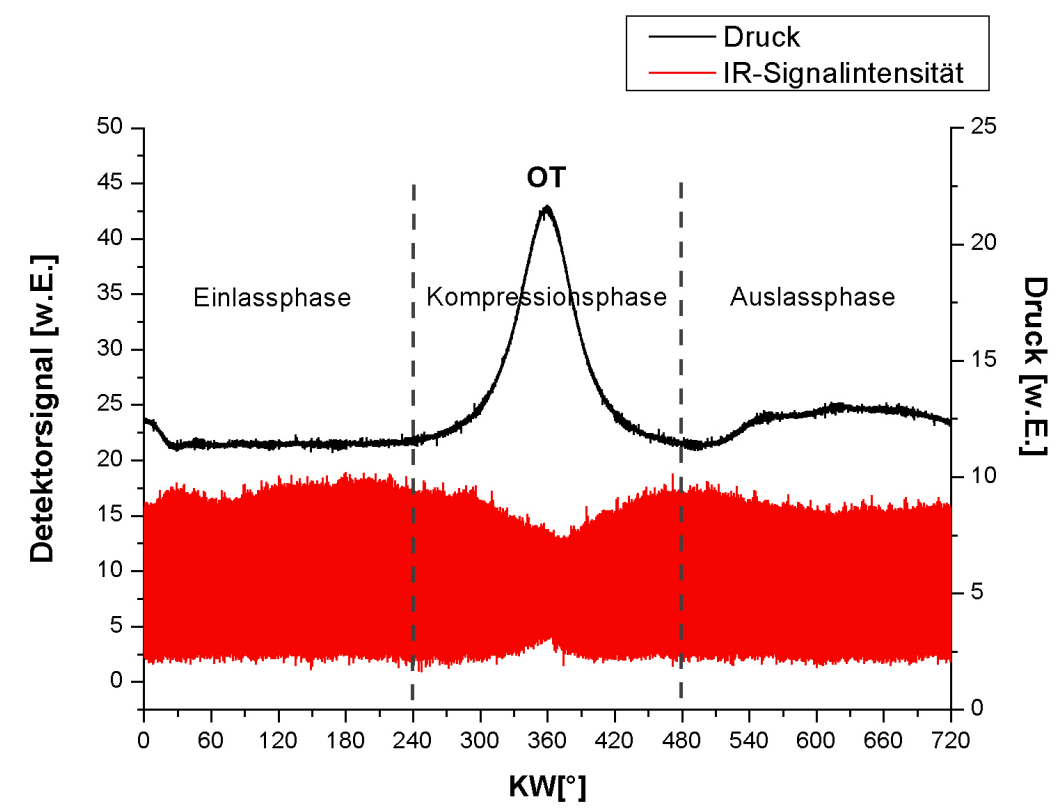

Abbildung 5.3.: Das IR-Signal (untere Kurve) und zugehöriger Zylinderdruck (obere Kurve) eines nicht gefeuerten Motorzyklus mit Einspritzung von Euro Super

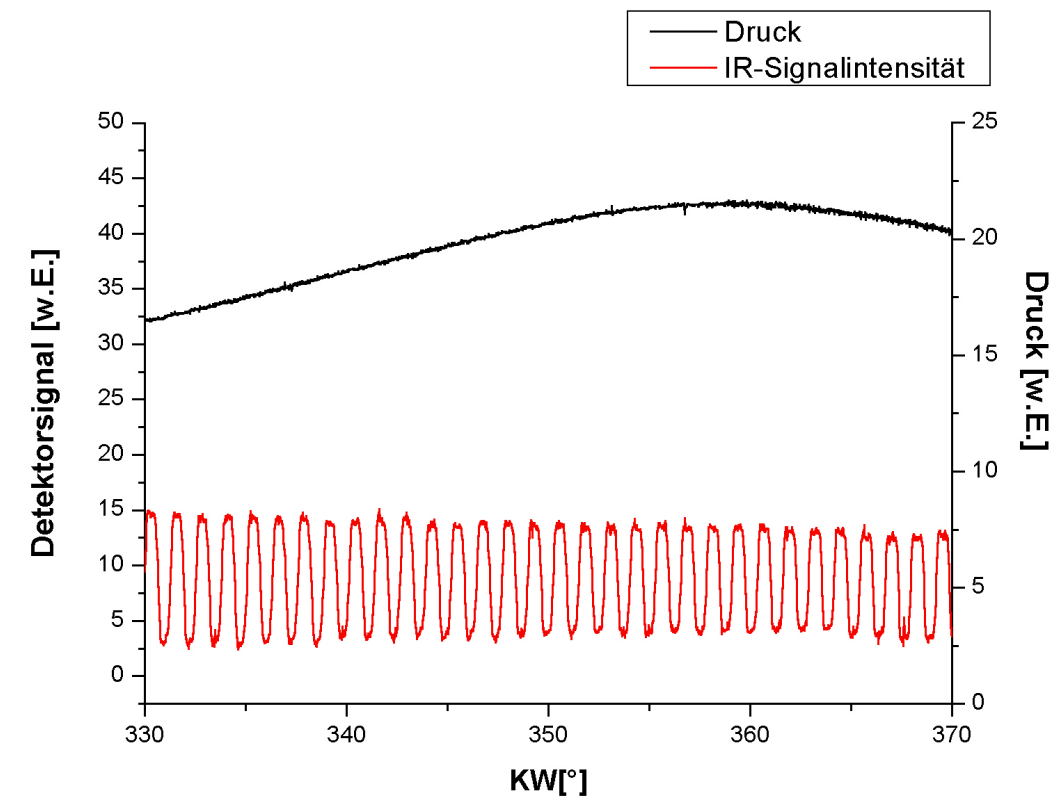

Abbildung 5.4.: Ausschnittsvergrößerung von Abbildung 5.3 zur Verdeutlichung des modulierten Detektorsignals 


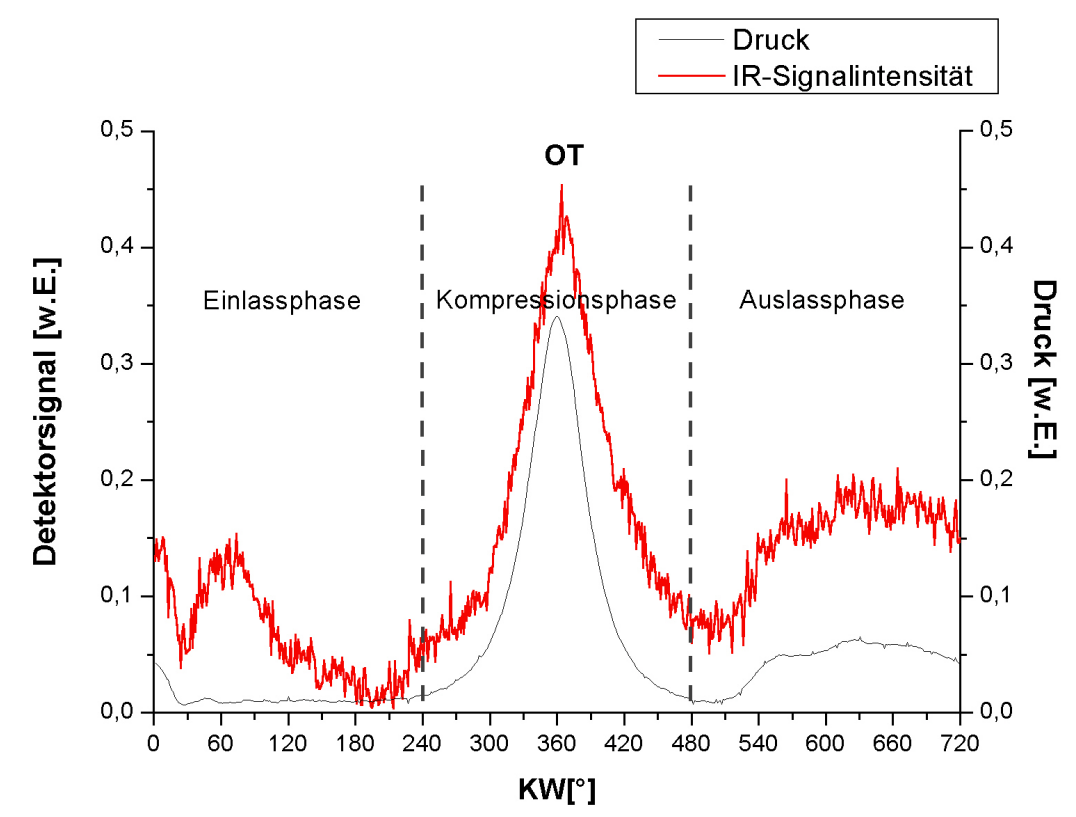

Abbildung 5.5.: Aus dem IR-Signal mit Hilfe des Software-Boxcars ermitteltes Absorptionssignal eines Motorzyklus mit Einspritzung von Euro Super

\subsubsection{Näherungsverfahren zur $\lambda$-Wert-Bestimmung}

Bei motorischen Untersuchungen zur Bestimmung der Güte der Gemischbildung ist der Lambda-Wert eine wichtige Größe (siehe Kapitel 2.1). Dafür müsste aber sowohl die Kraftstoffkonzentration, als auch die Sauerstoffkonzentration gleichzeitig bestimmt werden. Letztere lässt sich aber mit diesem Messsystem nicht ermitteln, da Sauerstoff ein homonukleares Molekül und daher Infrarot inaktiv ist. Es besteht aber die Möglichkeit aus der Absorptionsgleichung 3.29 und der Gleichung für ideale Gase ein physikalisches Näherungsverfahren zu entwickeln, um aus den Messdaten einen kurbelwinkelaufgelösten Verlauf des Lambda-Wertes gewinnen zu können.

In einem Luft/Kraftstoffgemisch ist die Kraftstoffkonzentration $C$ im durchstrahlten Volumen $V$ gegeben durch die Anzahl der absorbierenden Moleküle $v_{k}$ des Kraftstoffes in mol:

$$
C=\frac{v_{k}}{V}
$$

Mit dem Lambert-Beerschen Absorptionsgesetz ergibt sich dann:

$$
\ln \left(\frac{I}{I_{0}}\right)=-\sigma_{\lambda} \frac{v_{k}}{V} L
$$

Betrachtet man das Luft/Kraftstoffgemisch als ideales Gas, so wird der Zusammenhang zwischen Druck $p$ und Temperatur $T$ beschrieben durch

$$
p V=\left(v_{l}+v_{k}\right) R T
$$


mit $v_{l}$ mol Luft, $v_{k}$ mol Kraftstoff und der Gaskonstante $R=8,31 \mathrm{~J} \mathrm{~K}^{-1} \mathrm{~mol}^{-1}$. Bei einem zündfähigen Luft/Kraftstoffgemisch ist die Kraftstoffmenge $v_{k}$ klein im Vergleich zur angesaugten Luftmenge. Aus diesem Grund kann der Kraftstoff in der Gleichung des idealen Gases 5.3 vernachlässigt und nur die Luftmenge berücksichtigt werden. Der Einfluss von Druck und Temperatur auf das gemessene Absorptionssignal $I$ lässt sich durch Substitution von $V$ herausrechnen und es ergibt sich für den konzentrationsabhängigen Wert $\alpha$ :

$$
\alpha=\frac{-\ln \left(\frac{I}{I_{0}}\right)}{\frac{p}{T}}=\frac{\sigma_{\lambda} L}{R} \frac{v_{k}}{v_{l}}
$$

Als Verhältnis von $v_{k}$ und $v_{l}$ ist $\alpha$ dann vergleichbar mit einem relativen LambdaWert. Für eine absolute Bestimmung des $\lambda$-Wertes ist eine Kalibration mit bekannten Luft/Kraftstoffverhältnissen notwendig. Der Druck lässt sich experimentell durch einen zusätzlich im Brennraum installierten Druckaufnehmer bestimmen. Die lokale Temperatur im Messvolumen zu ermitteln, gestaltet sich sehr viel schwieriger, da die meisten Messtechniken schnelle Temperaturänderungen, wie sie im Brennraum auftreten, nicht registrieren oder diese Änderungen zu groß sind und daher die Strömungen des Gemischbildungsprozesses beeinflussen. Allerdings kann die Temperatur auch rechnerisch näherungsweise bestimmt werden (siehe Abschnitt 5.1.5).

Bei diesem Näherungsverfahren wird davon ausgegangen, dass sich nur reine Raumluft und Kraftstoff im Brennraum befindet. Restgase der vorherigen Verbrennung oder durch Abgasrückführung zugeleitete Gase werden dabei nicht berücksichtigt.

\subsubsection{Untersuchungen am Serienmotor}

Mit dem vorgestellten IR-Messsystem wurden erstmalig Untersuchungen an einem benzindirekteinspritzenden Otto-Serienmotor der Volkswagen AG (FSI 1,4l) durchgeführt. Um das Detektionssystem kalibrieren zu können, wurde der Motor homogen bei sieben verschiedenen definierten Lambda-Werten zwischen $\lambda=0,8$ und $\lambda=1,4$ betrieben. Da im Homogenbetrieb bei diesem Motorentyp erst kurz vor dem Zeitpunkt der höchsten Kompression tatsächlich ein homogenes und damit definiertes Gemisch vorliegt, wurde für die Kalibration nur das Absorptionssignal am oberen Totpunkt (OT) verwendet. Der Druck wurde synchron zu diesem aufgezeichnet und die Temperatur der Ansaugluft gemessen. Mit Hilfe der Adiabatengleichung

$$
\frac{T^{\prime}}{T}=\left(\frac{p^{\prime}}{p}\right)^{\frac{\kappa-1}{\kappa}}
$$

kann aus dem Druckverlauf und der Ansaugtemperatur die Gemischtemperatur für den gesamten Motorzyklus extrapoliert werden. Für den Adiabaten-Exponenten wird dabei $\kappa=1,32$ für Euro Super angenommen. Die Temperatur kann nur rechnerisch indirekt 
ermittelt werden, da es derzeit noch sehr schwierig ist, sie direkt im Brennraum kurbelwinkelaufgelöst zu messen.

Aus den gemessenen Absorptionssignalen, dem gemessenen Druck und der berechneten Temperatur bei den verschiedenen homogenen Betriebszuständen am OT können nun die zugehörigen $\alpha$-Werte bestimmt werden. Geht man davon aus, dass zu diesem Zeitpunkt der eingestellte Lambda-Wert am Messort (an der Zündkerze) auch tatsächlich vorliegt, so lässt sich eine Kalibrationskurve für das eingesetzte Messsystem erstellen (Abbildung $5.6)$.

Die beste Übereinstimmung mit den Messwerten wurde mit einer Funktion des Typs $a-b \cdot \ln x$ erzielt. Damit ergibt sich der Lambda-Wert aus dem errechneten Wert $\alpha$ durch:

$$
\lambda=a-b \cdot \ln (\alpha)=a-b \cdot \ln \left(-\frac{T}{p} \ln \left(\frac{I}{I_{0}}\right)\right)
$$

mit den Kalibrationskonstanten $a$ und $b$. Da für die Anpassung der Funktion nur LambdaWerte zwischen 0,8 und 1,4 im Homogenbetrieb zur Verfügung standen, sind aus den Messwerten berechnete $\lambda$-Werte außerhalb dieses Bereiches nur bedingt richtig.

Mit dem IR-Messsystem wurden eine Vielzahl von Betriebspunkten des Motors vermessen. Neben den sieben homogenen Betriebszuständen für die Kalibration des Messsystems, erfolgten fünf Messungen im geschichteten Betrieb, d.h. bei einer Einspritzung des Kraftstoffes während der Kompression, mit einer Variation des Ansteuerendes (ASE) des Injektors. Für jeden Betriebspunkt des Motors wurden jeweils 20 Zyklen vermessen. Nach Gleichung 5.6 wurden anschließend daraus die über diese Zyklen gemittelten druck- und temperaturkorrigierten Lambda-Werte berechnet. Die absolute Standardabweichung $s$ der

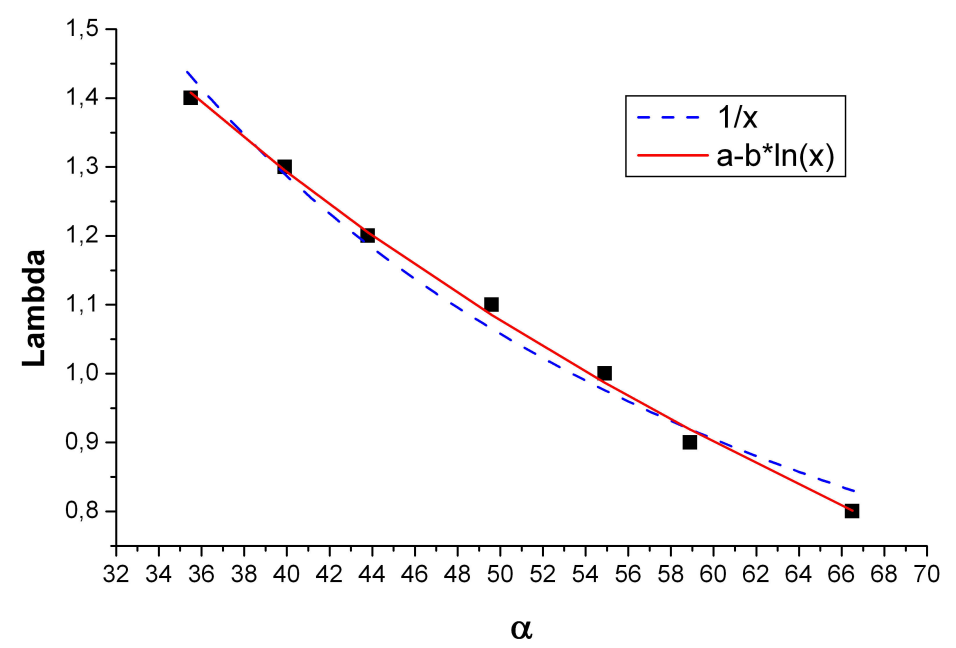

Abbildung 5.6.: Kalibrationskurve zur Bestimmung des Lambda-Wertes am Serienmotor 


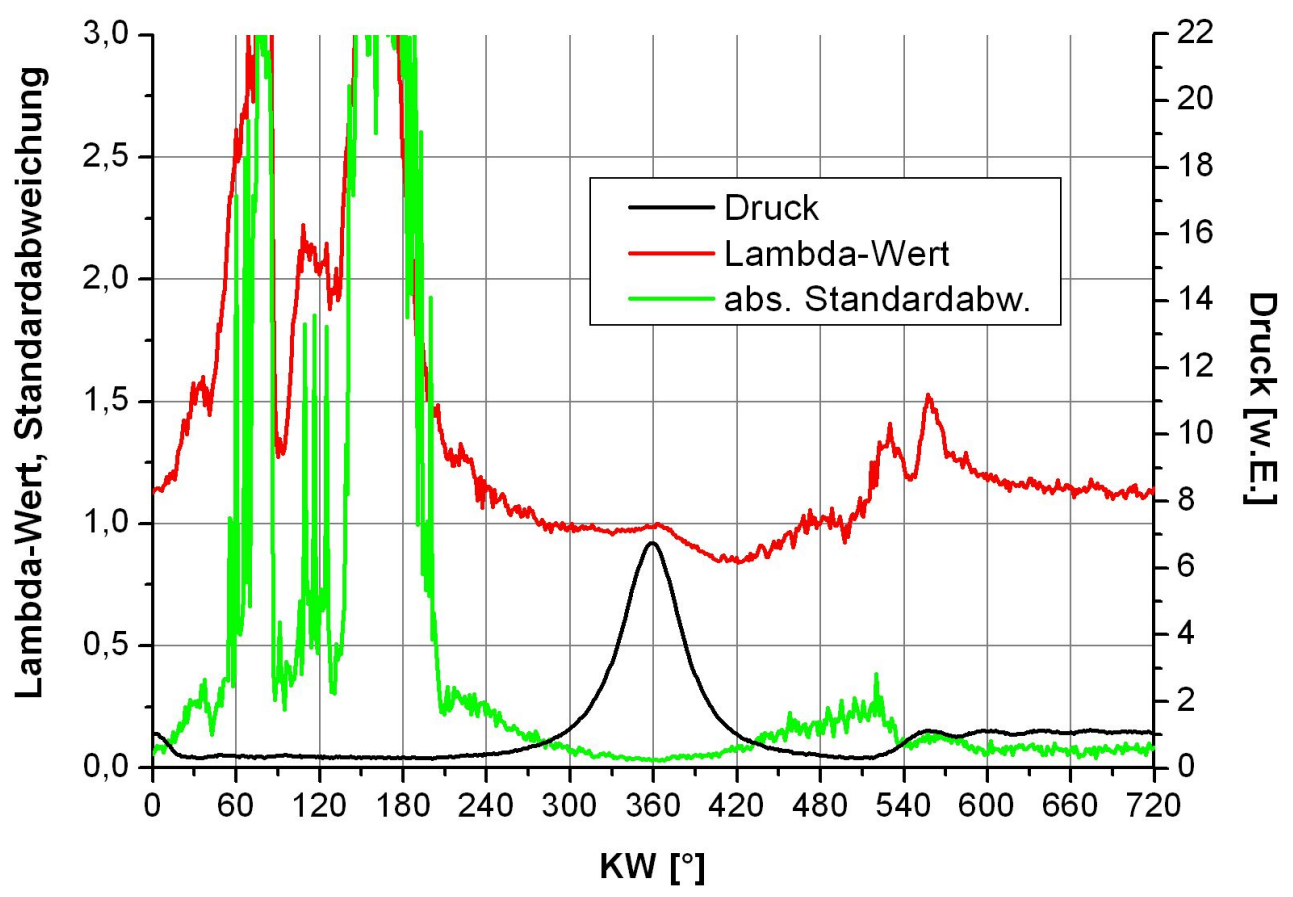

Abbildung 5.7.: Mit Hilfe des Näherungsverfahrens ermittelter Verlauf des Lambda-Wertes im homogenen Betrieb mit $\lambda=1,0$

$n$ Einzelzyklen vom gemittelten Lambda-Verlauf ist dabei

$$
s=\sqrt{\frac{1}{n-1} \sum_{i=1}^{n}\left(\lambda_{i}-\bar{\lambda}\right)^{2}} .
$$

In Abbildung 5.7 ist der mit Hilfe des Näherungsverfahrens gewonnene gemittelte Verlauf des Lambda-Wertes im Homogenbetrieb bei $\lambda=1$ dargestellt. Zur Verdeutlichung der Druckverhältnisse ist der Druck ebenfalls mit angegeben. Da der Motor ungezündet betrieben wird, ist bei $0^{\circ} \mathrm{KW}$ als Restgas noch unverbranntes Luft/Kraftstoffgemisch des vorherigen Zyklus im Brennraum vorhanden, welches durch die angesaugte Luft in der Einlassphase verdünnt und damit mager wird. Die Einspritzung beginnt bei etwa $80^{\circ} \mathrm{KW}$. Das Sensorsystem zeigt deutlich, dass Kraftstoff kurz nach dem Beginn an der Zündkerze vorhanden ist, da im Lambda-Verlauf das Gemisch kurzzeitig fetter wird. Nach dem Ende der Einspritzung wird der Kraftstoff durch nachströmende Luft wieder verdrängt und der Lambda-Wert steigt wieder. Ab $180^{\circ} \mathrm{KW}$ erfolgt die Kompression. Es zeigt sich, dass sich bereits bei $60^{\circ} \mathrm{KWvOT}\left(300^{\circ} \mathrm{KW}\right)$ die Luft und der Kraftstoff homogen gemischt haben, da der $\lambda$-Wert von diesem Zeitpunkt an bis zum OT nahezu konstant bleibt.

Das entwickelte Sensorsystem besitzt keine Möglichkeit das Gemisch zu zünden. Daher ist auch nach OT Kraftstoff im Brennraum vorhanden und das Gemisch wird sogar noch fetter, der Lambda-Wert nimmt ab. Dieser Effekt tritt auch auf, wenn der Motor gefeuert 


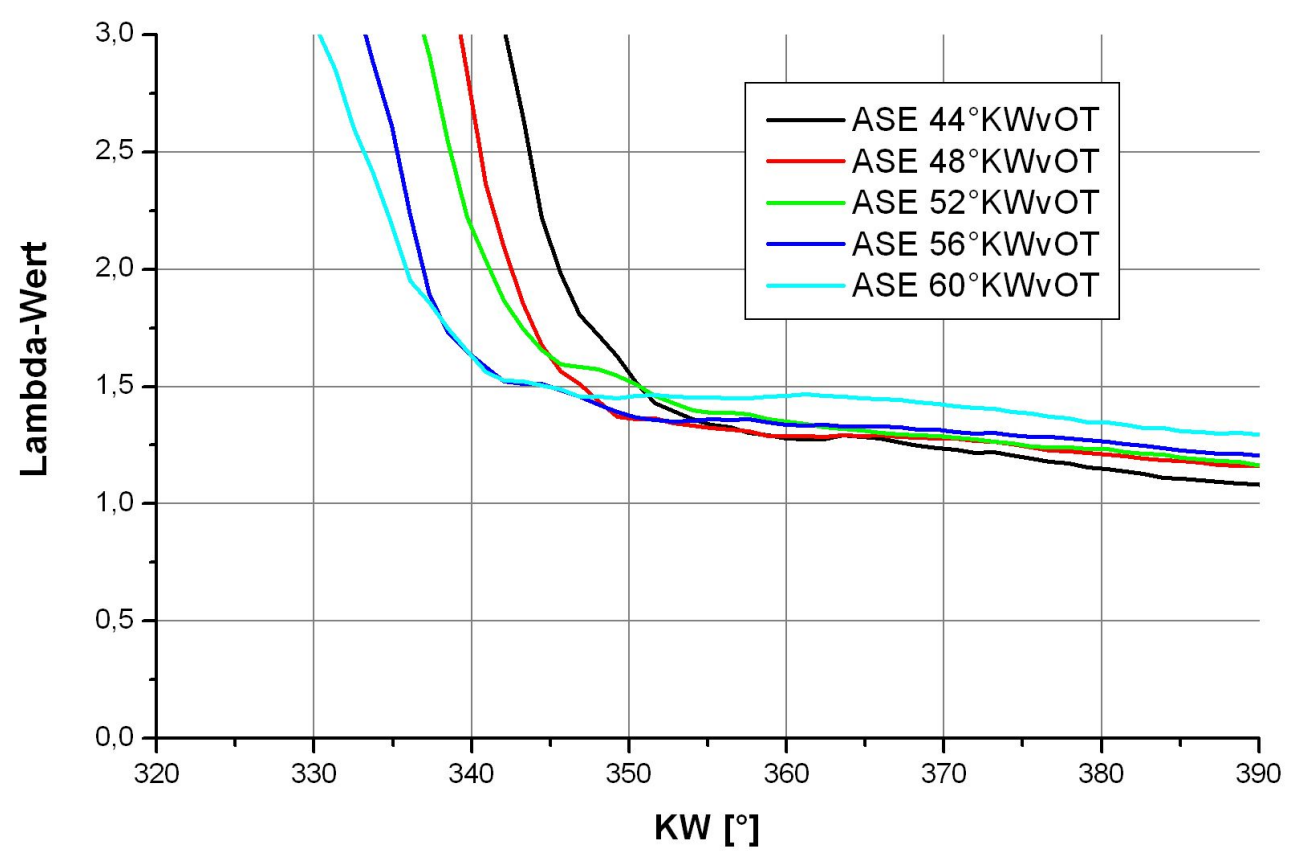

Abbildung 5.8.: Variation des Ansteuerendes der Einspritzung im geschichteten Betrieb

betrieben wird und rührt von den Flammstegen, der Kolbenmulde und den Brennraumwänden ausgasendem Kraftstoff her [33]. Da auch nach dem Auslass immer noch Restgas im Brennraum verbleibt, ist noch bis zum nächsten Zyklus ein Kraftstoffgemisch detektierbar.

Die Standardabweichung ist vor und nach der Einspritzung sehr groß und fluktuiert stark. Der Grund dafür ist, dass für diese Kurbelwinkel nahezu kein Kraftstoff im Messvolumen vorliegt und daher bei der Berechnung der absoluten Standardabweichung Rauschanteile überproportional eingehen. Bei den Messungen im Homogenbetrieb liegt die Standardabweichung in der Kompression um den OT unterhalb von 0,1. Sie enthält dabei tatsächliche Zyklusschwankungen sowie Messfehler, die durch das Detektorrauschen verursacht werden.

Die Messungen im geschichteten Betrieb des Motors zeigen wie erwartet, dass sich die Variation des Ansteuerendes (ASE) der Einspritzung im zeitlichen Verlauf des über 20 Zyklen gemittelten Lambda-Wertes bemerkbar macht (siehe Abbildung 5.8). Je früher die Einspritzung beendet ist, desto früher lässt sich auch ein fetter werdendes Gemisch detektieren.

Die tatsächlichen Zyklusschwankungen, welche sich in der Standardabweichung widerspiegeln, unterscheiden sich zwischen homogenen und geschichteten Betriebspunkten (Abbildung 5.9). Vergleicht man nur die Absorption von aufeinander folgenden Zyklen, so zeigt sich ein deutlicher Einfluss des Einspritzzeitpunktes auf die Gemischbildung. 

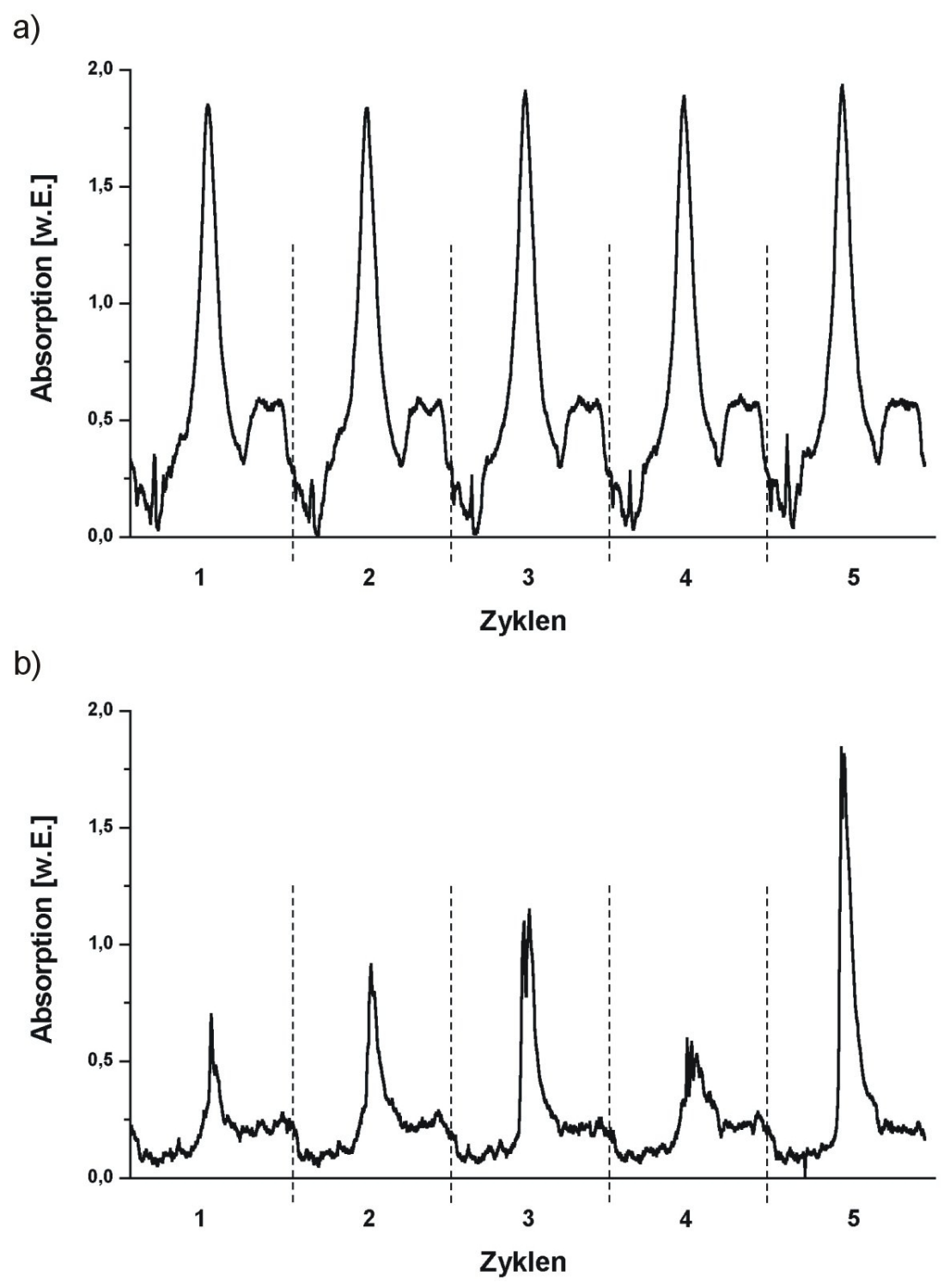

Abbildung 5.9.: Absorptionssignal in aufeinander folgenden Zyklen a) homogener Betrieb $(\lambda=1,0)$ b) geschichteter Betrieb $\left(\mathrm{ASE}=53^{\circ} \mathrm{KWvOT}\right)$

Durch die frühe Einspritzung des Kraftstoffs im homogenen Betrieb (um $270^{\circ} \mathrm{KWvOT}$, Lambda $=1,0$ ) besteht genügend Zeit für die Verdampfungsprozesse, so dass die Zyklus-zuZyklus-Schwankungen in der Kraftstoffkonzentration gering sind, wie in Abbildung 5.9a deutlich wird. Beim geschichteten Betrieb dagegen, beispielsweise mit ASE $=53^{\circ} \mathrm{KWvOT}$ (Abbildung 5.9b), sind die Verdampfungsprozesse des Kraftstoffs noch nicht beendet und die thermischen Bedingungen variieren. Es zeigen sich deutliche Zyklusschwankungen im Absorptionssignal und damit in der Kraftstoffkonzentration. 


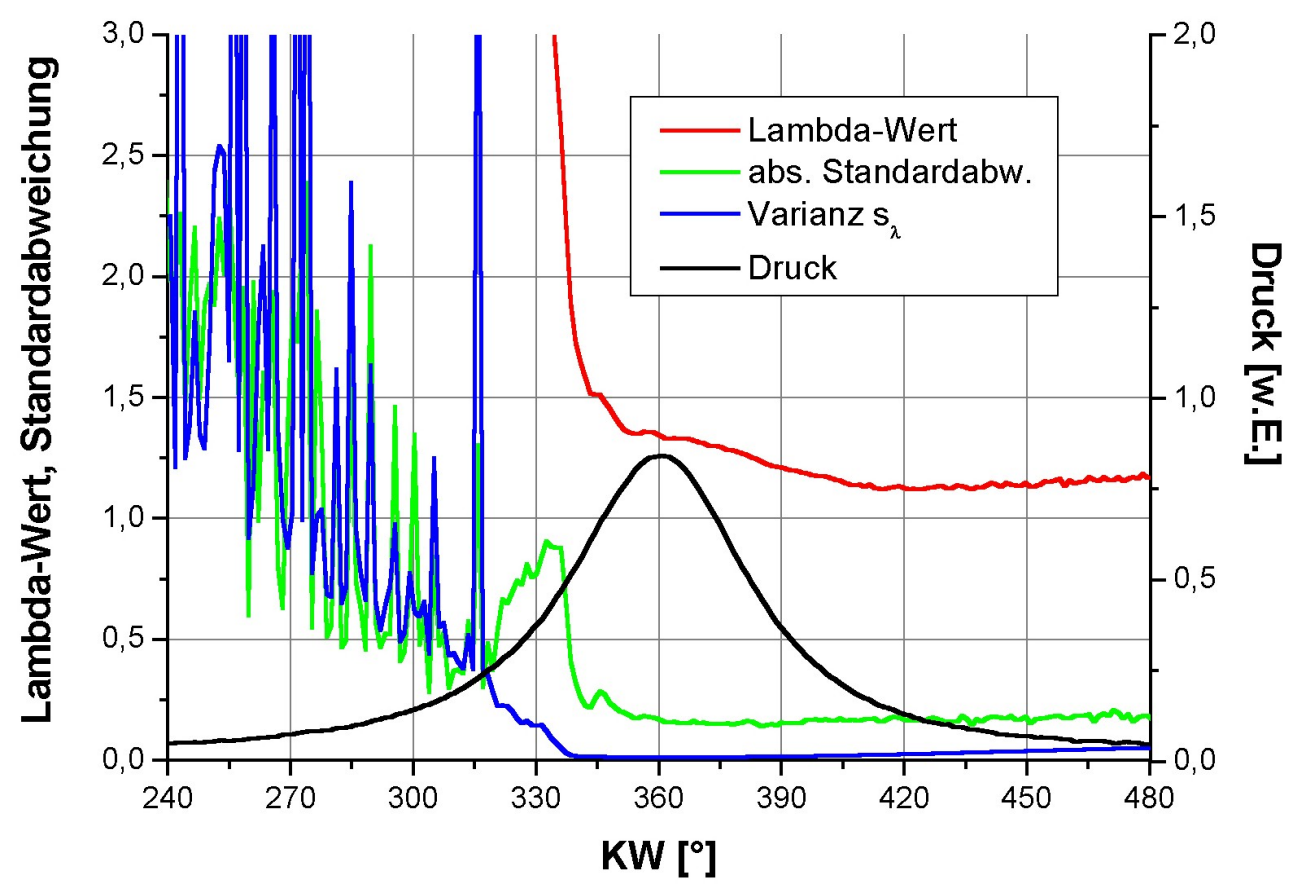

Abbildung 5.10.: Verlauf des Lambda-Wertes im geschichteten Motorbetrieb (ASE $\left.=56^{\circ} \mathrm{KW}\right)$ sowie die Gesamtstandardabweichung und der Fehler des Detektionssystems

\subsubsection{Fehlerbetrachtung}

Sehr geringe Kraftstoffmengen verursachen nur ein sehr kleines Absorptionssignal, welches u.U. im Signalrauschen untergeht. Dieses Rauschen setzt sich hauptsächlich zusammen aus thermischem Rauschen des HgCdTe-Detektors, der sehr empfindlich auf Wärmestrahlung reagiert, und dem elektronischen Rauschen des Verstärkersystems. Um den Einfluss des Rauschens der Detektorspannung $I$ auf die Berechnung der Lambda-Werte zu ermitteln, kann aus der Fehlerfortpflanzung die Varianz der Standardabweichung $s_{\lambda}$ des $\lambda$-Wertes in Abhängigkeit von der Detektorspannung berechnet werden:

$$
s_{\lambda}^{2}=\left(\frac{\partial s(I)}{\partial I}\right)^{2} \cdot s_{i}^{2}=\left(-\frac{b}{I \cdot \ln \left(\frac{I}{I_{0}}\right)}\right)^{2} \cdot s_{i}^{2}
$$

Hierbei ist $s_{i}$ die Standardabweichung der Spannung des Detektionssystems. In Abbildung 5.10 ist der Verlauf des Lambda-Wertes im geschichteten Betrieb zum Zeitpunkt der Kompression sowie die absolute Standardabweichung und deren Varianz dargestellt. Das Ende der Einspritzung liegt hier bei $\mathrm{ASE}=56^{\circ} \mathrm{KWvOT}$.

Es zeigt sich, dass die aus den Einzelzyklen ermittelte Standardabweichung bis etwa $315^{\circ} \mathrm{KW}$ durch die Spannungsschwankungen des Detektors bestimmt wird. Erst dann 


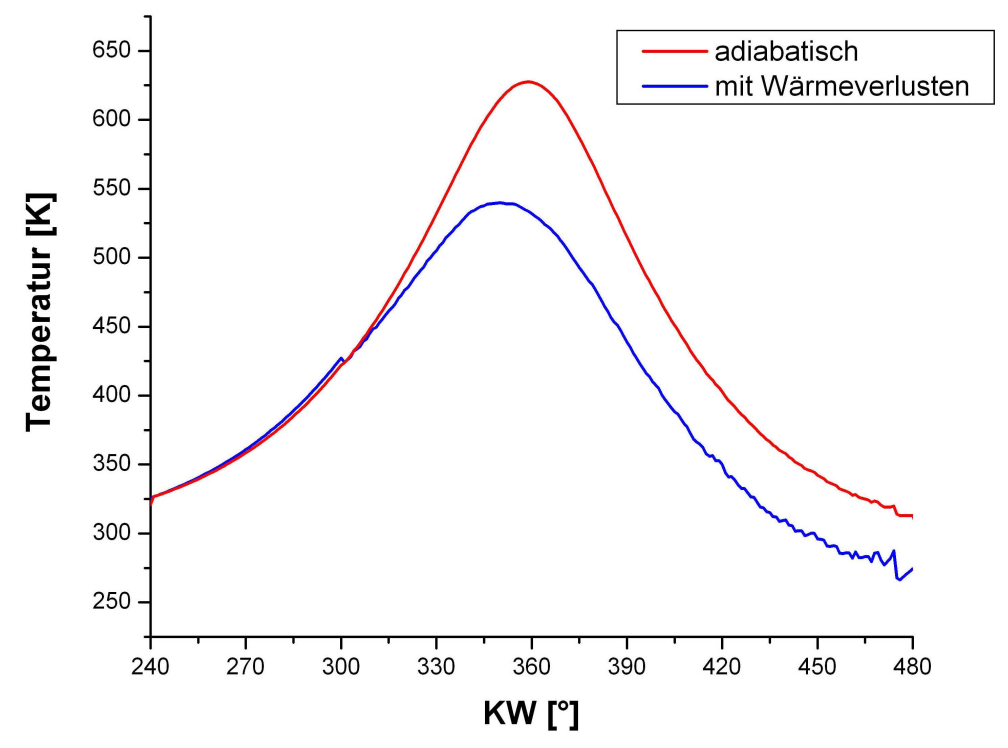

Abbildung 5.11.: Aus den gleichen Anfangsbedingungen berechnete Temperaturverläufe des Luft/Kraftstoffgemisches während der Kompression, einmal nach der Adiabatengleichung, das andere Mal unter Berücksichtigung von Wärmeverlusten

führen tatsächlich zyklische Schwankungen in der Gemischbildung bei etwa $330^{\circ} \mathrm{KW}$ zu einer erhöhten Standardabweichung. Liegt also Kraftstoff am Ort der Zündkerze vor, so beeinflusst das Rauschen des Detektionssystems die ermittelten Lambda-Werte nicht mehr wesentlich.

Eine weitere Ungenauigkeit bei der Bestimmung des Lambda-Wertes ergibt sich durch die nicht über den gesamten Motorzyklus gemessene Temperatur. Bei dem eingesetzten Näherungsverfahren wird diese nach der Adiabatengleichung 5.5 berechnet. Unberücksichtigt bleibt dabei allerdings der Einfluss verschiedener im Brennraum auftretender Effekte auf die tatsächliche lokale Temperatur, wie beispielsweise das Abkühlen des Gemisches durch den verdampfenden Kraftstoff oder Wandwärmeverluste an den auf etwa $80^{\circ} \mathrm{C}$ gekühlten Wänden des Brennraums.

Wie groß der Unterschied in der Temperatur durch diese Vorgänge ist, wird durch die in Abbildung 5.11 gezeigten Temperaturverläufe deutlich. Bei der ersten Berechnung der Temperatur wurde ein adiabatisches Verhalten des Gemisches angenommen. Die Spitzentemperatur am OT beträgt etwa 630K. Die zweite Temperaturkurve ist das Ergebnis von Simulationsrechnungen der Volkswagen AG. Dabei wurden die oben erwähnten Verlustkanäle mitberücksichtigt. In diesem Fall beträgt die höchste Temperatur nur etwa 540K. Beiden Berechnungen liegen die gleichen Anfangsbedingungen zu Grunde.

Diese Ungenauigkeit in der Bestimmung der Temperatur führt zu einer Ungenauigkeit in der Berechnung des absoluten $\lambda$-Wertes. Die in Abbildung 5.12 dargestellten Verläufe des Lambda-Wertes zeigen, dass schon kleine Temperaturvariationen einen Einfluss 


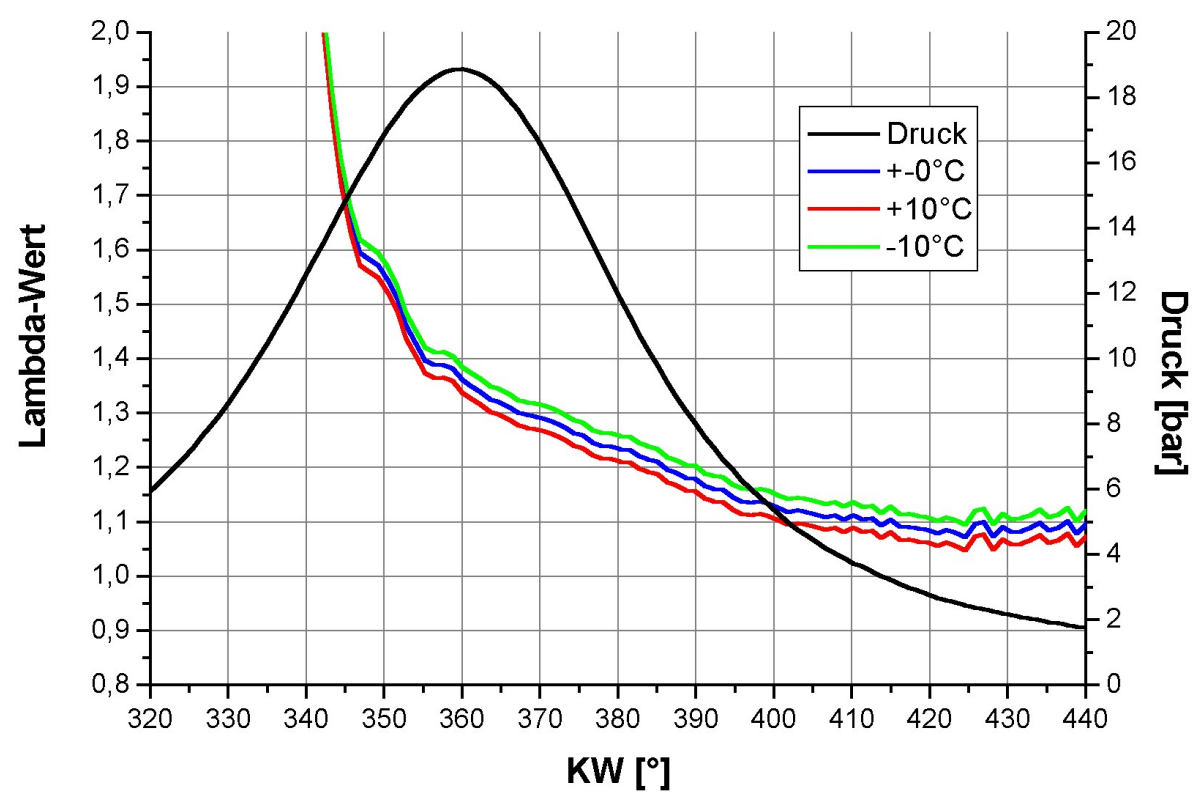

Abbildung 5.12.: Änderung des berechneten Lambda-Wertes mit der angenommenen Temperatur (geschichteter Betrieb, $\mathrm{ASE}=52^{\circ} \mathrm{KWvOT}$ )

auf den mit Hilfe des Näherungsverfahrens ermittelten Wert haben. Für den geschichteten Betriebspunkt mit $\mathrm{ASE}=52^{\circ} \mathrm{KWvOT}$ wurde für die Entwicklung der Temperatur ein adiabatisches Verhalten des Gemisches angenommen. Anschließend wurde bei der Berechnung des Lambda-Wertes die ermittelte Temperatur um $10^{\circ} \mathrm{C}$ variiert, woraus sich eine Änderung des Lambda-Wertes um etwa 0,02 ergibt.

Für eine exakte Lambda-Wert-Bestimmung wäre also eine sehr genaue Kenntnis der lokalen Temperatur oder der tatsächlichen Sauerstoffkonzentration notwendig, wie dies z.B. in den in Kapitel 4 beschriebenen Untersuchungen zur spontanen Ramanstreuung der Fall ist. 


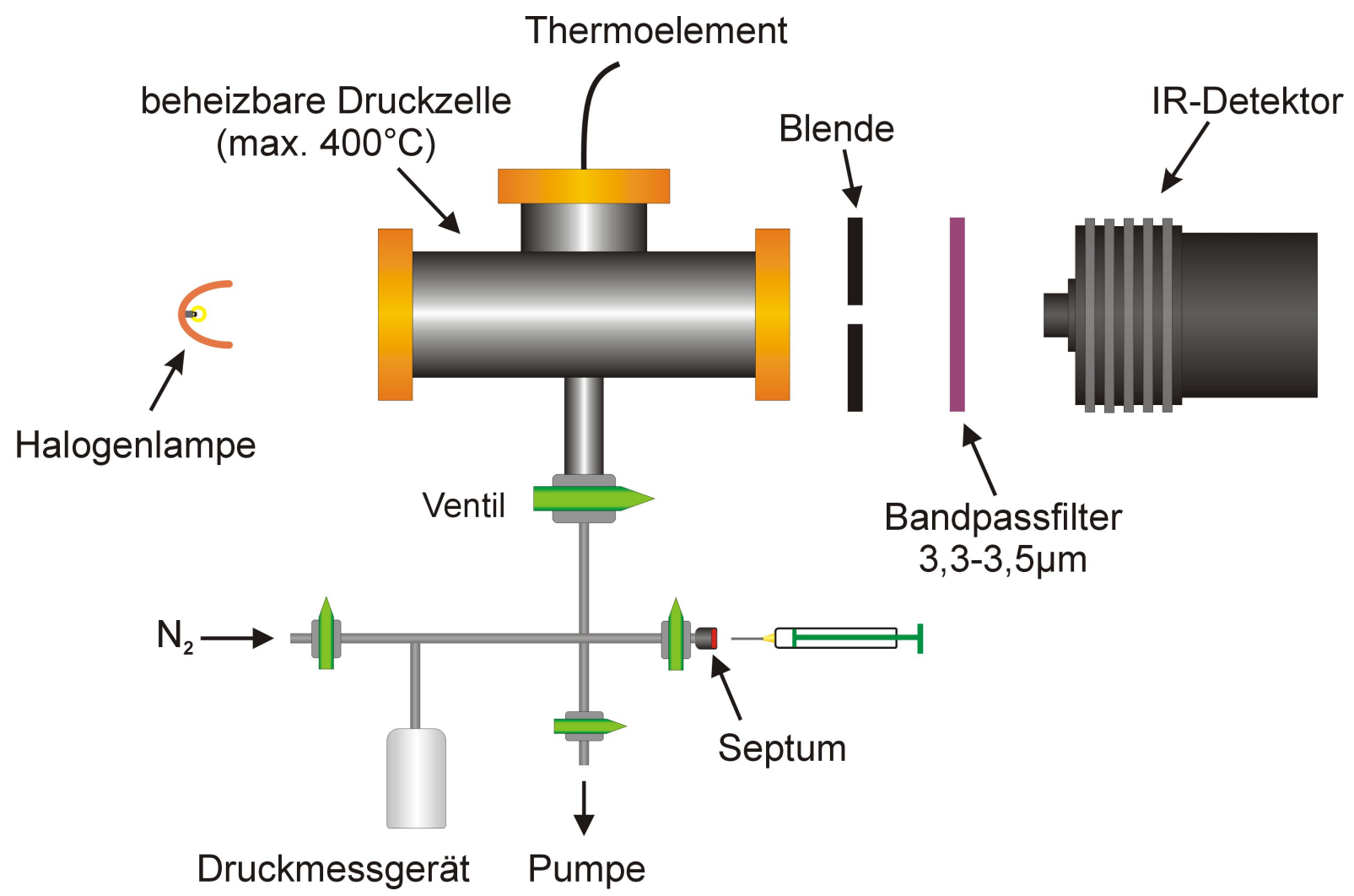

Abbildung 5.13.: Experimenteller Aufbau zur Untersuchung der Abhängigkeit der Absorption von der Temperatur des Gemisches

\subsubsection{Temperatureinfluss auf IR-Absorption}

Im vorherigen Abschnitt wurde der Einfluss der Temperatur auf die Genauigkeit der Lambda-Wert-Bestimmung durch das Näherungsverfahren erläutert. Da sich die Besetzung der Vibrations- und Rotationszustände der Kohlenwasserstoffmoleküle mit der Temperatur ändert (siehe Abschnitt 3.3.2), hängt der Absorptionskoeffizient einer Molekülspezie bei einer festen Wellenlänge zusätzlich von der Temperatur ab.

In Abbildung 5.14 sind die Transmissionskurven zweier für die Absorptionsmessungen verwendeter spektraler Filter zusammen mit der skalierten Absorptionskurve von Euro Super dargestellt. Dabei zeigt sich, dass der „schmale“ Filter (SF) mit einer spektralen Breite von etwa $150 \mathrm{~nm}$ die Absorptionsbande des Kraftstoffs nicht vollständig umfasst. Anders ist dies bei dem „breiten“ Filter (BF) mit einer Halbwertsbreite von etwa 300nm. Eine Umbesetzung der Rotationszustände innerhalb des Grundschwingungszustandes infolge einer Temperaturänderung wird sich also nur beim Einsatz des „schmalen“ Filters bemerkbar machen. 


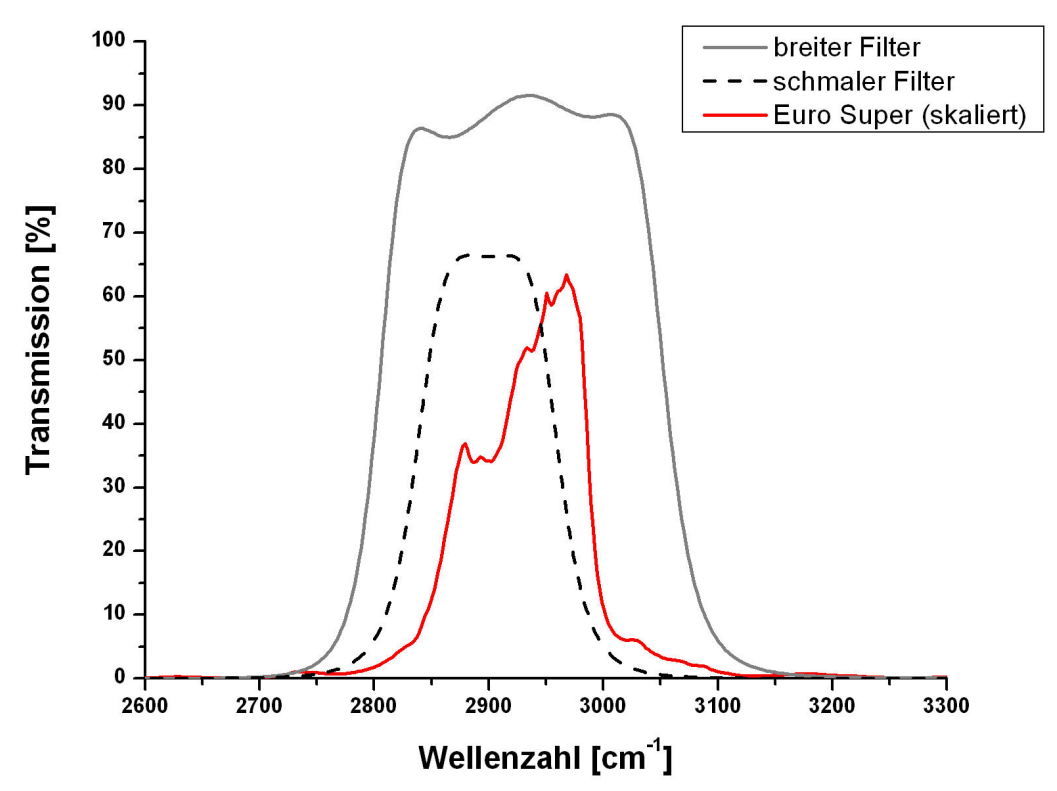

Abbildung 5.14.: Transmissionsspektrum der beiden verwendeten IR-Filter zusammen mit der (skalierten) Absorptionsbande von Euro Super

Um den Einfluss der Temperatur auf die durch das Transmissionsspektrum der Filter bestimmten Absorptionskoeffizienten zu untersuchen, wurde ein in Abbildung 5.13 skizzierter experimenteller Aufbau realisiert. Dieser bestand aus einer mit MagnesiumfluoridFenstern ausgestatteten Druckzelle, welche bis zu einer Temperatur von $400^{\circ} \mathrm{C}$ heizbar war. Als Lichtquelle wurde die bereits eingesetzte Halogenlampe verwendet. Eine Blende vor dem $\mathrm{HgCdTe}$-Detektor gewährleistete, dass keine Wärmestrahlung von den Wänden der Zelle detektiert wurde. Die Gemischtemperatur wurde mit Hilfe eines Thermoelements im Inneren der Zelle bestimmt. Die beiden Bandpassfilter ließen sich vor dem Detektor ohne weitere Justage positionieren.

Untersucht wurden die Absorptionseigenschaften von Euro Super bei verschiedenen Temperaturen. Dazu wurde die Druckzelle jeweils bei einer eingestellten Temperatur zunächst evakuiert. Mit jedem der beiden Filter konnte so erst das Gesamtsignal und anschließend das reine Hintergrundsignal gemessen werden. Letzteres entstand aus der thermischen Strahlung der Zelle und ließ sich durch Blocken der IR-Strahlung der Halogenlampe ermitteln. Die Differenz dieser beiden Signale ergibt jeweils die eingestrahlte Intensität $I_{0}$. Anschließend wurde die Zelle langsam mit Stickstoff befüllt. Bei diesen Messungen wurde aus Sicherheitsgründen Stickstoff verwendet, um keine zündfähigen Gemische zu erzeugen. Über ein Septum konnte gleichzeitig mit einer Spritze dem Stickstoffstrom in die Zelle Kraftstoff beigemengt werden. Die Menge betrug immer etwa 0,5ml Euro Super. Um zu gewährleisten, dass sich das Gemisch in einem thermischen Gleichgewicht befindet, wurde in mehreren Schritten über einige Minuten der Gesamtdruck langsam auf 1bar 


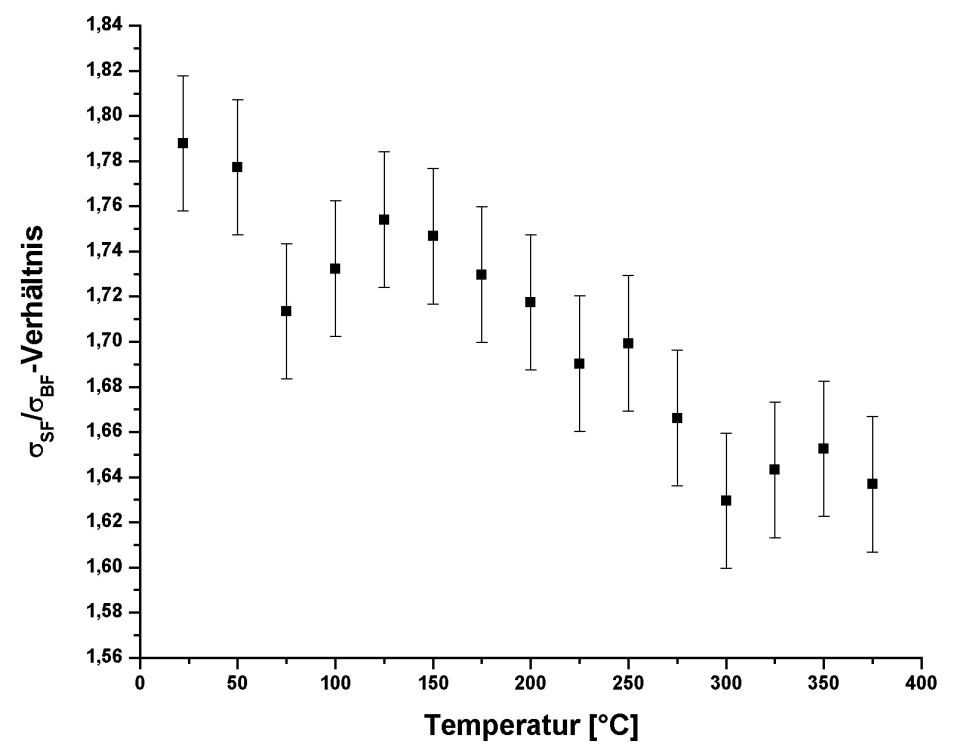

Abbildung 5.15.: Verhältnis $\sigma_{S F} / \sigma_{B F}$ der Absorptionskoeffizienten der verwendeten Filter bei verschiedenen Temperaturen

erhöht. Die Messungen wurden immer bei gleichem Druck durchgeführt, um einen reinen Temperatureinfluss nachweisen zu können.

Nachdem die Zelle bei einer festen Temperatur befüllt worden war, wurde wieder mit jedem Filter erst das Gesamt- und dann das Hintergrundsignal gemessen, um so das Absorptionsignal $I \mathrm{zu}$ ermitteln. Aus Gleichung 3.29 ergibt sich aus der Absorption der beiden Filter das Verhältnis der Absorptionskoeffizienten des „schmalen“ Filters $\sigma_{S F}$ zum Absorptionskoeffizienten des ,breiten“ Filters $\sigma_{B F}$ :

$$
\frac{\ln \left(\frac{I_{S F}}{I_{0, S F}}\right)}{\ln \left(\frac{I_{B F}}{I_{0, B F}}\right)}=\frac{\sigma_{S F}}{\sigma_{B F}}
$$

Dieses Verhältnis ist in Abbildung 5.15 für verschiedene Temperaturen dargestellt. Es zeigt sich dabei, dass das Verhältnis nicht konstant ist, sondern mit der Temperatur abnimmt. Benzin besitzt auf Grund der Vielzahl an Komponenten keinen eigentlichen Siedepunkt, sondern einen Siedebereich zwischen $30^{\circ} \mathrm{C}$ und etwa $180^{\circ} \mathrm{C}$ (siehe Anhang, Prüfbericht Euro Super). Das Siedeende liegt bei $184^{\circ} \mathrm{C}$. Höhersiedende Komponenten können daher ebenfalls Ursache für eine Änderung des Verhältnisses der Absorptionskoeffizienten bis zu dieser Temperatur sein. Beispielsweise hat der aromatische Kohlenwasserstoff Benzol, einer der Bestandteile von Euro Super, einen Siedepunkt von $80^{\circ} \mathrm{C}$. Bei dieser Temperatur ändert sich das Verhältnis deutlich (siehe Abbildung 5.15). Oberhalb der Siedeendetemperatur kann aber beim „schmalen“ Filter eine Änderung des Absorptionskoeffizenten $\sigma_{S F}$ nur durch eine Umbesetzung der Rotationszustände mit steigender Temperatur erfolgen. 
Diese Untersuchungen zeigen, dass es bei den Absorptionsmessungen notwendig ist, einen Bandpassfilter zu verwenden, der spektral die gesamte Absorptionsbande der Substanz umfasst. Andernfalls verfälschen Temperatureinflüsse eine genaue Konzentrationsbestimmung. Denn auch bei Substanzen mit vielen Komponenten und einer breiten Absorptionsbande, wie dies bei Benzin der Fall ist, ändert sich der Absorptionskoeffizient bei einer Wellenlänge mit der Temperatur.

Für die Bestimmung der Kraftstoffdichte im mittleren Infrarot um 3,4 $\mu \mathrm{m}$ ist es möglich auch andere Lichtquellen als die hier verwendete Halogenlampe einzusetzen. Beispielsweise haben Nishiyama et al. [42, 43] einen Helium-Neon-Laser bei einer Wellenlänge von $3,392 \mu \mathrm{m}$ in motorischen Untersuchungen verwendet. Allerdings ist dann eine Kalibration des Detektionssystems für den jeweils eingesetzten Kraftstoff notwendig, um Temperatureinflüsse auf eine Konzentrationsbestimmung zu eliminieren [59]. 


\subsection{Spektroskopische Bestimmung der Sauerstoffdichte}

Die im vorherigen Abschnitt beschriebenen Experimente zeigen, dass die Kenntnis der tatsächlichen Sauerstoffkonzentration am Ort der Messungen zur genaueren Bestimmung des Lambda-Wertes notwendig ist. Dieser lässt sich durch die nur schwer bestimmbare lokale Temperatur mit dem vorgestellten Näherungsverfahren auch nur ungenau ermitteln. Sauerstoff als homonukleares Molekül besitzt allerdings kein Dipolmoment und daher auch keine Absorptionslinien im mittleren infraroten Wellenlängenbereich.

Im nahen Infrarot allerdings besitzen $\mathrm{O}_{2}$ Moleküle schwache, elektronisch Dipol verbotene Übergänge (siehe Abschnitt 3.3.3). Die Linienstärke dieser Übergänge sind von der Größenordnung $10^{-23}-10^{-25} \mathrm{~cm} /$ Molekül [49]. In diesem Abschnitt soll daher eine Messtechnik vorgestellt werden, die mit Hilfe eines Vertical Cavity Surface-Emitting Lasers (VCSEL-Laser) bei 763nm eine Bestimmung der Sauerstoffkonzentration ermöglicht. Durch die Schmalbandigkeit des Lasers ist es mit Hilfe einer Wellenlängenmodulation möglich, einzelne Rotationslinien der $\mathrm{O}_{2}$-Moleküle zu detektieren.

Üblicherweise werden bei Laserabsorptionsmessungen mechanische Chopper und LockIn-Verstärker eingesetzt, um das Signal/Rausch-Verhältnis zu verbessern. Ein Nachteil solcher Systeme in motorischen Anwendungen ist die geringe Zeitauflösung von LockIn-Verstärkern. Das in diesen Experimenten eingesetzte Detektionssystem basiert daher auf einer besonders rauscharmen Version eines Differenzverstärkers, welches von Hobbs ursprünglich für Wellenlängenmultiplex-Kommunikationsanwendungen entwickelt wurde $[26,27]$ und eine hohe Zeitauflösung ermöglicht.

Leider konnten mit dem Messsystem nur einführende Messungen erfolgen, da der eingesetzte VSCEL-Laser der Firma Laser Components nach wenigen Stunden Betrieb ausfiel.

\subsubsection{VCSEL-Laser}

Bei den Untersuchungen zur Bestimmung der Sauerstoffkonzentration wurde ein VCSELLaser der Firma Laser Components (OXY760) mit einer Wellenlänge um 760nm eingesetzt. Bei diesem war die Laserdiode direkt an eine für diese Wellenlänge singlemodige Glasfaser gekoppelt. Die spektrale Bandbreite des Lasers betrug etwa 30MHz. Um eine hohe spektrale Auflösung zu gewährleisten, wurden in dem gesamten experimentellen Aufbau Singlemode-Glasfasern verwendet. Der Strom und die Temperatur der Diode wurden mittels eines Lasertreibers der Firma Tektronix (ITC502) geregelt, wobei an dem Gerät zusätzlich die Möglichkeit bestand, den Diodenstrom extern über eine Kontrollspannung zu verändern $(20 \mathrm{~mA} / \mathrm{V})$. Die emittierte Wellenlänge des VCSEL-Lasers ist abhängig sowohl von der Temperatur der Laserdiode als auch vom Strom. Die Temperatur betrug bei den Messungen $35^{\circ} \mathrm{C}$ und konnte auf $0,01^{\circ} \mathrm{C}$ genau stabilisiert werden. Der Strom 
wurde über die Kontrollspannung mit Hilfe eines Funktionsgenerators mit einer Frequenz von $1 \mathrm{kHz}$ zwischen $3,4 \mathrm{~mA}$ und 5,4mA moduliert. Dies entspricht einer Modulation im Wellenlängenbereich zwischen etwa 759,5nm und 760,2nm. Eine genaue Wellenlängenbestimmung war bei den Untersuchungen nicht möglich, so dass eine Bestimmung nur anhand der Spezifikationen der Diode erfolgen konnte.

\subsubsection{Signaldetektion}

Die Laserdetektion erfolgte bei den Experimenten mittels eines speziellen Differenzverstärkers, einem sog. Balanced Ratiometric Detection Systems (BRD-System) [26, 27]. Ein schematischer Schaltplan ist in Abbildung 5.16 dargestellt. Das System besitzt zwei Si-Photodioden, eine für das Absorptionssignal und eine für ein Referenzsignal. Es ist in der Lage, analog aus den Strömen der beiden Photodioden das Laserrauschen herauszurechnen und das elektronische Rauschen $\mathrm{zu}$ minimieren. Dabei werden die Ströme $I_{\text {sig }}$ und $I_{\text {comp }}$ der Signaldiode und der Referenzdiode am Operationsverstärker $A_{1}$ von einander abgezogen. Um einen DC Differenzstrom von Null zu erhalten, erfolgt eine Rückkopplung des Differenzstromes automatisch über den Operationsverstärker $A_{2}$. Wird der eingesetzte Laserstrahl also aufgeteilt und der Referenzstrahl direkt und der Signalstrahl nach einer Absorption auf den Detektor geleitet, so wird genau das auf beiden Signalen gleichermaßen vorhandene Rauschen des Lasers abgezogen. Am linearen Ausgang ist dann das Hochpass-gefilterte Absorptionssignal messbar.

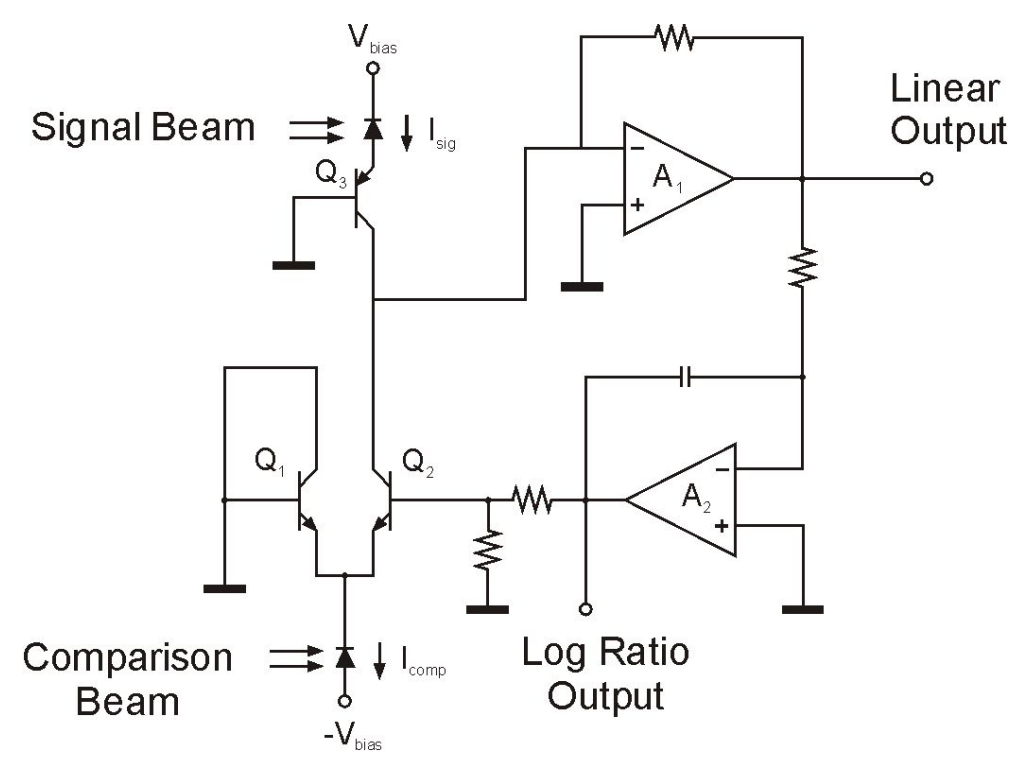

Abbildung 5.16.: Schematischer Schaltplan des BRD-Systems (Balanced Ratiometric Detection System) 


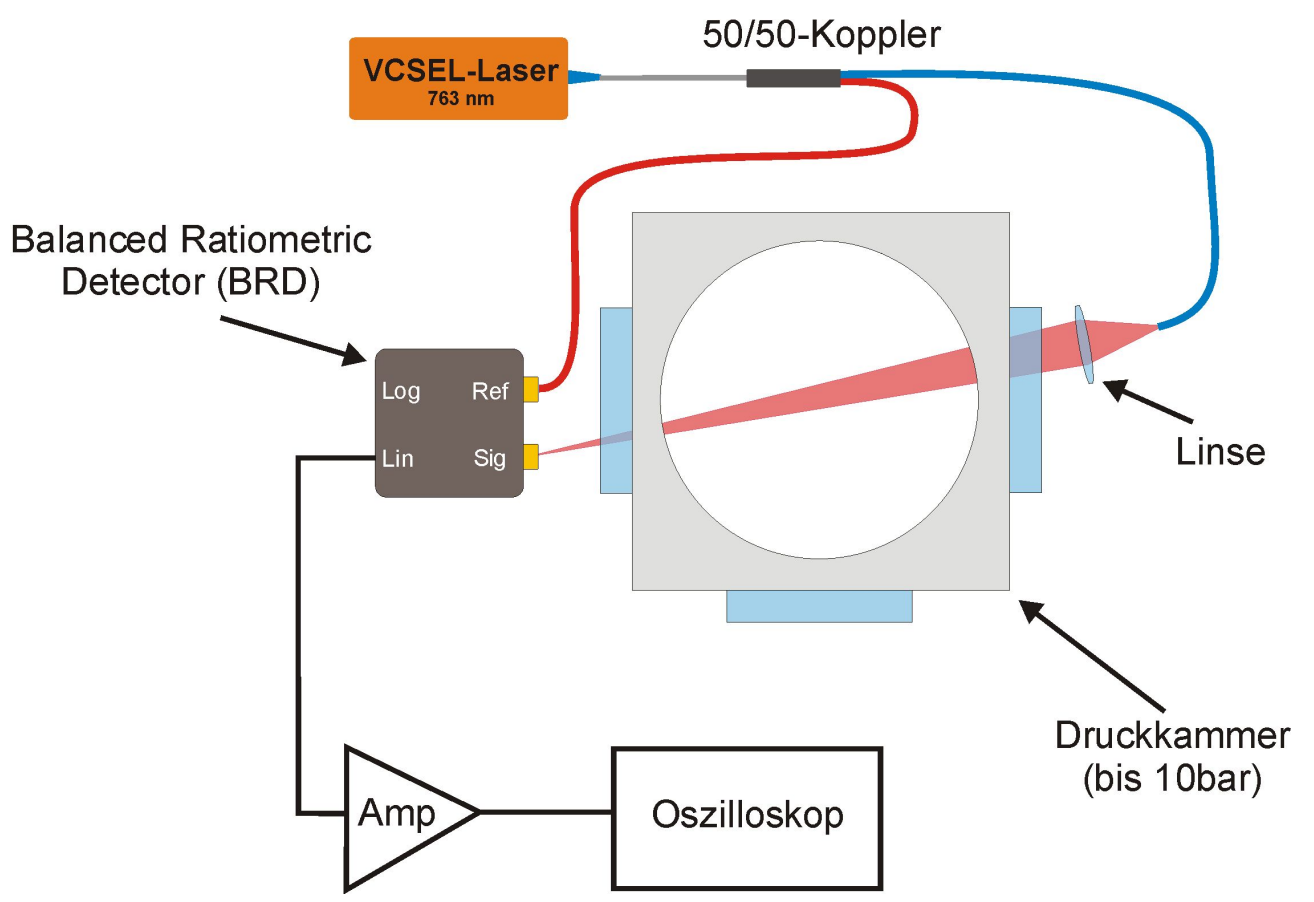

Abbildung 5.17.: Experimenteller Aufbau zur Bestimmung der Sauerstoffkonzentration

Durch die Rückkopplung ergibt sich die Tiefpass-gefilterte Spannung des logarithmischen Verhältnisses der beiden Strahlen am logarithmischen Ausgang zu

$$
V_{l o g}=-\ln \left(\frac{I_{\text {ref }}}{I_{\text {comp }}}-1\right)
$$

Eine Rauschunterdrückung von bis zu 50dB ist mit diesem System möglich. Die Frequenzbandbreite des BRD-Systems ist an die Bandbreiten der Si-Photodioden und der Operationsverstärker gebunden. Bei dem hier eingesetzten System liegt sie aber oberhalb von $100 \mathrm{kHz}$ für den linearen Ausgang (siehe Anhang für Liste der eingesetzten Komponenten). So ist eine hohe Zeitauflösung bei motorischen Anwendungen möglich. Daher wurde bei allen Untersuchungen das lineare Detektorausgangssignal ausgewertet. Der logarithmische Ausgang ist für die Detektion sehr kleiner Signale besser geeignet, allerdings ist er generell nur für Messungen bei niedrigen Modulationsfrequenzen bis etwa $100 \mathrm{~Hz}$ einsetzbar.

\subsubsection{Experimenteller Aufbau}

Der bei der Bestimmung der Sauerstoffkonzentration verwendete experimentelle Aufbau ist in Abbildung 5.17 skizziert. Das vom VCSEL-Laser kommende Licht wurde zunächst in einem 50/50-Faserkoppler aufgeteilt. Der eine Teil wurde direkt mittels einer Glasfaser auf 
die Referenz-Photodiode des BRD-Systems geleitet. Der andere Teil des Laserlichts wurde mit einer Linse durch eine Druckkammer hindurch auf die Signal-Photodiode abgebildet. Dabei durchlief der Laserstrahl die Druckkammer unter einem schrägen Einfallswinkel, um Etaloneffekte durch die Fenster der Kammer zu minimieren. Mit Hilfe eines Spannungsverstärkers (Femto - DLPVA-100) wurde die vom Detektionssystem erzeugte Spannung des linearen Ausgangs verstärkt und von einem Oszilloskop aufgezeichnet.

Die Hochdruckkammer war für Drücke bis 10bar ausgelegt. Für die Experimente bei Raumtemperatur wurde die Druckkammer nach dem Evakuieren mit synthetischer Luft befüllt.

\subsubsection{Druckvariation}

Da sich beim VCSEL-Laser mit steigendem Diodenstrom nicht nur die Wellenlänge des emittierten Lichts ändert, sondern auch die Leistung steigt, wird auch das detektierte Signal größer. In Abbildung 5.18 ist das aufgezeichnete Rohsignal des Detektors für eine ansteigende Modulationsflanke bei einem Kammerdruck von 1,2bar dargestellt. Dabei entspricht der niedrigste Spannungswert von etwa -2,7V der Leistung beim höchsten Diodenstrom, da der invertierte Eingang am Operationsverstärker $A_{1}$ verwendet wurde.

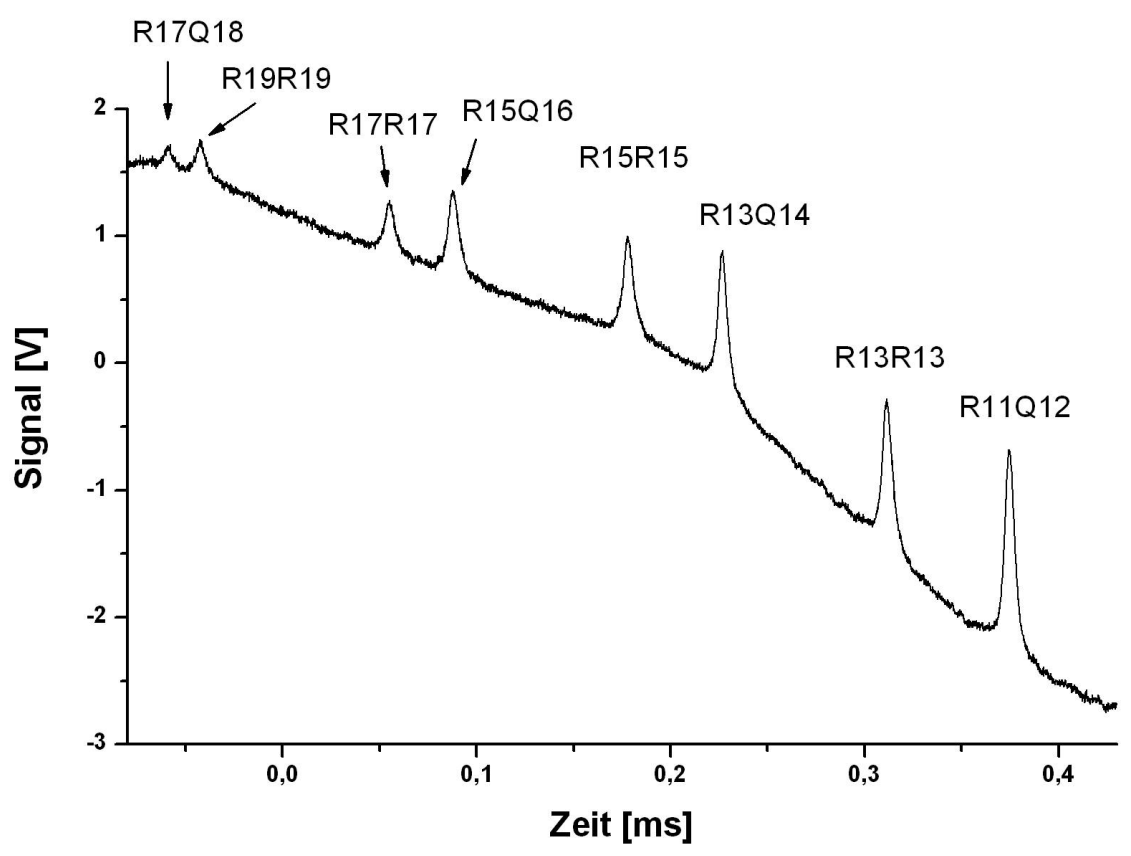

Abbildung 5.18.: Sauerstoff-Rohsignal des Detektors am Oszilloskop bei Wellenlängenmodulation (Kammerdruck=1,2bar) 


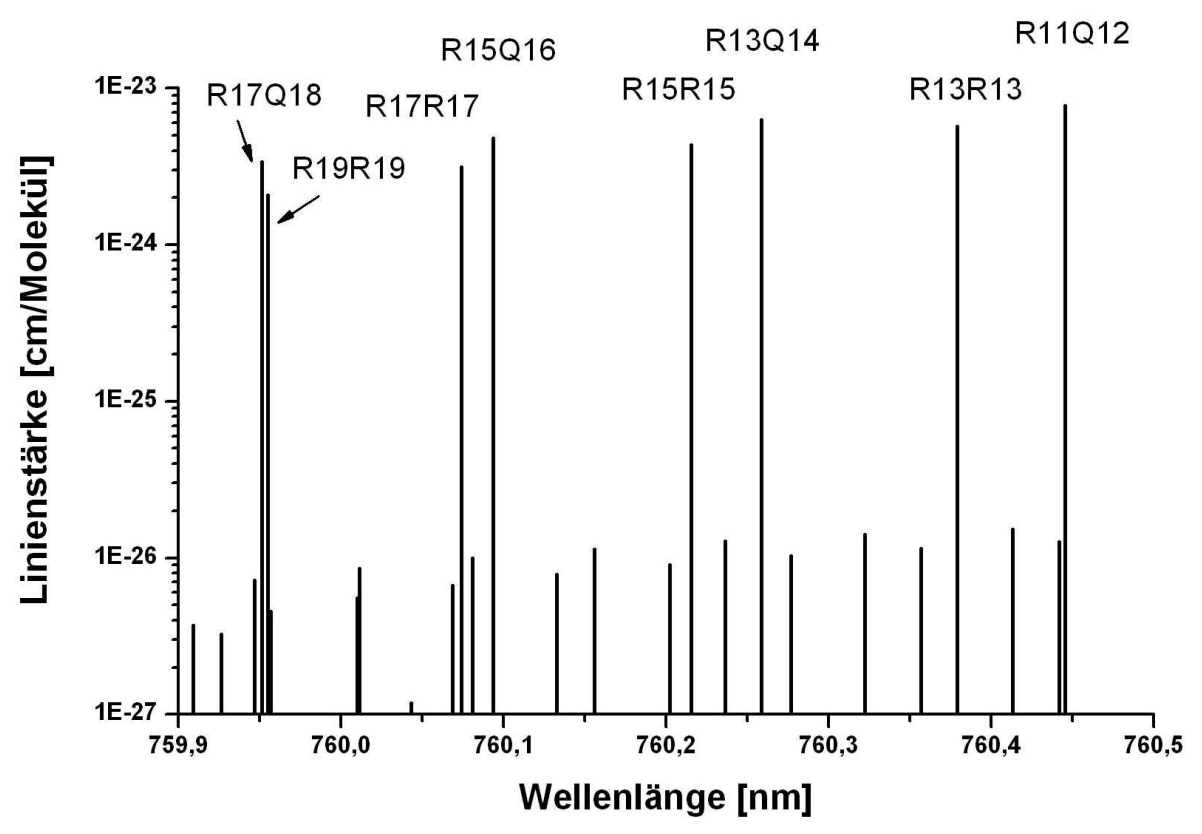

Abbildung 5.19.: Absorptionslinien von Sauerstoff im untersuchten Wellenlängenbereich (berechnet mit HITRAN database [49])

Das Detektorsignal ist in Abbildung 5.18 über der Modulationszeit aufgetragen. Diese Darstellung entspricht durch die ansteigende Modulationsflanke auch einer zunehmenden Wellenlänge. Deutlich zeigen sich verschiedene Rotationslinien des Sauerstoffs als positive Peaks. Da aber ein Teil des Absorptionsweges aufbaubedingt durch die Raumluft vor und nach der Druckkammer führt, sind die Absorptionssignale aus der Luft in der Druckkammer allerdings immer durch den Sauerstoff in der Raumluft außerhalb der Kammer überlagert. Um das reine Absorptionssignal vom Weg in der Zelle zu erhalten, kann das gemessene Signal bei evakuierter Zelle davon abgezogen werden. Ansonsten ließe sich diese von der Raumluft herrührende Absorption auch durch evakuierte oder Inertgasgespülte (beispielsweise $\mathrm{N}_{2}$ oder Ar) Druckkammervorbauten verhindern [60].

Bei den Experimenten zeigten sich einige Probleme mit dem eingesetzten VSCELLaser. Zum einen war die emittierte Leistung nicht liniear zum angelegten Diodenstrom. Dies zeigt sich auch in Abbildung 5.18 darin, dass die Signalintensitätsveränderung trotz einer linearen Strommodulation nicht linear ist. Damit verbunden ist eine nichtlineare Änderung der Wellenlänge, wodurch eine genaue Wellenlängenzuordnung erschwert wird. Das zweite Problem war, dass Rückreflexe von der Einkoppelfaser in die Laserdiode zu Störungen führten. Bemerkbar macht sich dies durch zeitliche Schwankungen in der relativen Position der Absorptionslinien zueinander (Jitter).

Das mit Hilfe der HITRAN database [49] berechnete Absorptionsspektrum von Sauerstoff im untersuchten Wellenlängenbereich, welcher aus den Spezifikationen des Lasers ermittelt werden kann, ist in Abbildung 5.19 dargestellt. Die relativen Positionen der Ab- 
sorptionslinien im Spektrum zeigen eine sehr gute Übereinstimmung zu den detektierten Linien, so dass diesen eine Wellenlänge zugeordnet werden kann.

Eine Änderung des Luftdrucks in der Kammer führt zu einer veränderten Linienform der detektierten Rotationslinien. Mit steigendem Druck wird die Halbwertsbreite größer (siehe Abschnitt 3.3.2). Dies ist in Abbildung 5.20 für die gemessenen Rotationslinien R15R15 und R13Q14 dargestellt. Der spektrale Abstand der Linien ist so gering, dass bei Drücken oberhalb von 2 bar die Druckverbreiterung zum Überlappen der Linien führt. Um die Spektren trotz der Schwankungen der Laserleistung vergleichen zu können, erfolgte deren Ausrichtung an der R13Q14-Linie. Insbesondere bei niedrigen Drücken ist zu beobachten, dass die Schwankungen zu Änderungen des relativen spektralen Abstandes zwischen den Linien führen.

Das Integral über das Spektrum dieser beiden Rotationslinien ergibt die Stärke der Absorption in diesem Spektralbereich und besitzt einen eindeutigen Zusammenhang zum Luftdruck in der Druckzelle (Abbildung 5.21). Mit dem Detektionssystem können geringe Sauerstoffkonzentrationen von weniger als 100ppm bei etwa 13cm Absorptionsstrecke bestimmt werden.

Mit dem vorgestellten Detektionssystem können geringe Sauerstoffkonzentrationen auch im Brennraum eines Ottomotors bestimmt werden. Die Empfindlichkeit des BRDSystems ermöglicht es, Sauerstoff unter atmosphärischen Bedingungen und einer Absorptionsstrecke von nur 1mm zu detektieren [4]. Damit ist der Einsatz eines solchen Systems auch unter motorischen Bedingungen möglich. Durch eine Kombination des in Abschnitt 5.1 vorgestellten Messsystems zur Bestimmung der Kraftstoffkonzentration mit dem Sauerstoffdetektionssystem könnte der exakte Lambda-Wert am Ort der Zündkerze bestimmt werden. Eine genaue Bestimmung der lokalen Temperatur wäre dafür nicht notwendig. Diese lässt sich aber zusätzlich durch die Detektion der Rotationslinien des Sauerstoffs auf zwei verschiedene Arten bestimmen. Zum einen kann die Temperatur ermittelt werden mit Hilfe einer Anpassung eines temperaturabhängigen Lorentz- oder Gauss-Profils (siehe Abschnitt 3.3.2) an eine der gemessenen Rotationslinien. Allerdings wird dies bei hohen Drücken durch die Überlappung der verbreiterten Rotationslinien erschwert. Eine zweite Möglichkeit besteht darin, die Temperatur über die Verhältnisse der Linienstärken verschiedener Rotationslinien zu bestimmen [46]. Denn die Besetzung der Rotationszustände ist temperaturabhängig und damit auch die Stärke ihrer Absorption. 


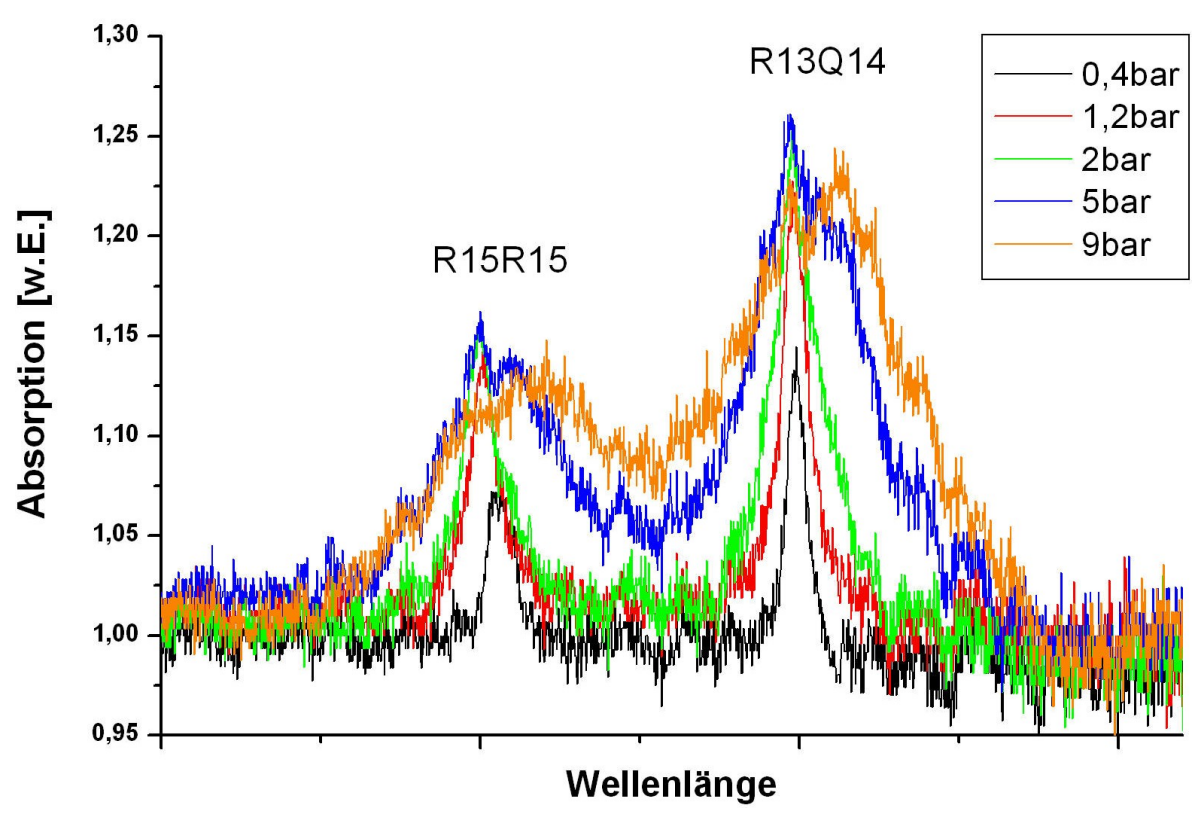

Abbildung 5.20.: Zunahme der Halbwertsbreite der Sauerstoff-Rotationslinien mit steigendem Druck

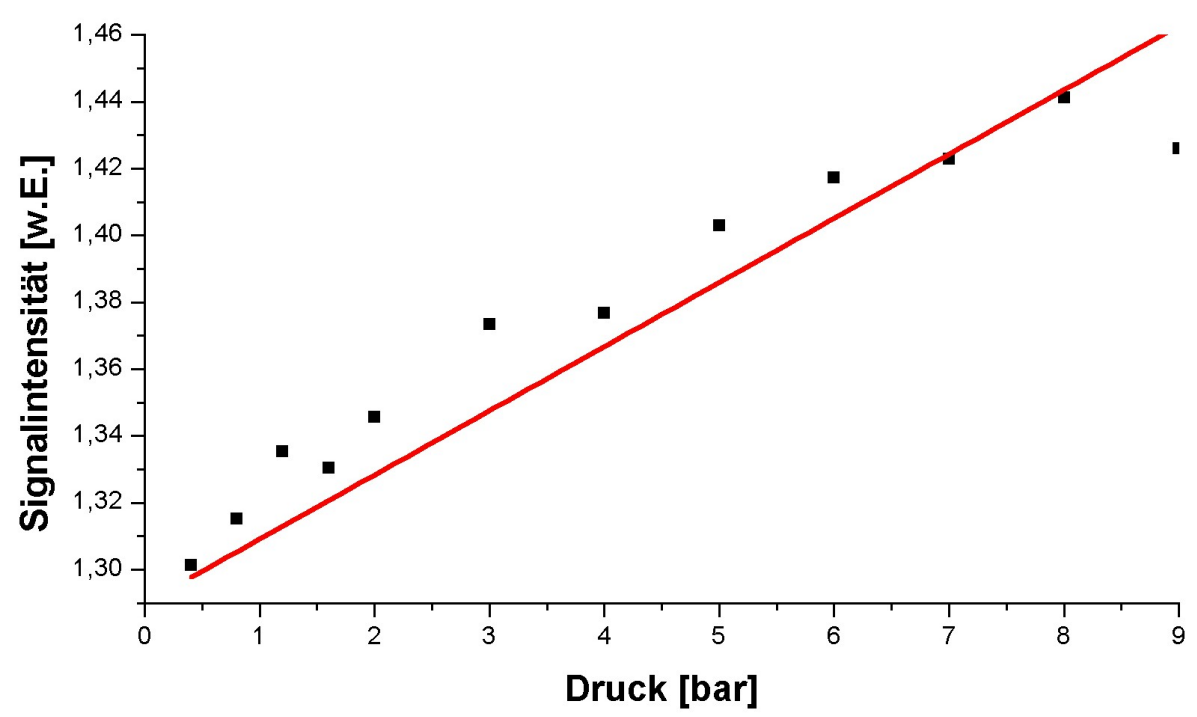

Abbildung 5.21.: Abhängigkeit der Linienstärke der beiden gemessenen Rotationslinien R15R15 und R13Q14 vom Luftdruck in der Druckzelle 


\subsection{Zusammenfassung und Ausblick}

Die genaue Kenntnis über die Gemischbildungsprozesse und deren zeitliche Entwicklung in einem Motorzyklus ist notwendig für eine Optimierung bezindirekteinspritzender Brennverfahren. Eine Messtechnik für eine zyklusaufgelöste Bestimmung der Kraftstoffkonzentration am Ort der Zündkerze wurde daher im vorliegenden Kapitel vorgestellt. Die in Kraftstoff enthaltenen Kohlenwasserstoffe absorbieren Licht im infraroten Wellenlängenbereich um 3,4 $\mu \mathrm{m}$ auf Grund von Vibrationsübergängen der CH-Schwingung. Bei dem im Rahmen dieser Arbeit entwickelten Messsystem wird das intensitätsmodulierte Licht einer Halogenlampe über Saphirfasern in einen Zündkerzenadapter geleitet. Eine kurze Absorptionsstrecke in diesem Adapter ermöglicht die Bestimmung der Kraftstoffkonzentration über die Änderung der Lichtintensität im spektralen Wellenlängenbereich um $3,4 \mu \mathrm{m}$. Es wurde ein Näherungsverfahren vorgestellt, mit dem der $\lambda$-Wert aus den Absorptionssignalen näherungsweise bestimmt werden kann. Untersuchungen an einem Serienmotor unter Verwendung von Euro Super als Kraftstoff zeigten die Einsatzfähigkeit dieses Systems unter motorischen Bedingungen und machten erstmals eine Bestimmung des Lambda-WertVerlaufs in einzelnen Motorzyklen möglich. Dabei zeigten sich deutliche Schwankungen des $\lambda$-Wertes in den geschichteten Betriebspunkten, welche eine Folge starker Variationen des Gemischbildungsprozesses durch den spät im Zyklus eingespritzten Kraftstoff sind. Es konnte gezeigt werden, dass für eine korrekte Bestimmung der Kraftstoffkonzentration eine spektral die gesamte Absorptionsbande des Kraftstoffs umfassende Detektion notwendig ist, da sonst die von der Temperatur abhängige Besetzung der Rotationsniveaus einen Einfluss auf das Messergebnis hat. Ein Vorteil dieser Messtechnik ist, dass einfache Lichtquellen, wie Halogenlampen, verwendet werden können anstelle von kostenintensiven Lasern.

Ein weiteres Detektionssystem für die Bestimmung der Sauerstoffkonzentration wurde in diesem Kapitel vorgestellt, welches durch den Einsatz eines schmalbandigen VCSELLasers bei einer Wellenlänge um 760nm in der Lage ist, Rotationslinien der $\mathrm{O}_{2}$-Moleküle spektral aufzulösen. Dabei werden sehr empfindliche Absorptionsmessungen erst durch eine besondere Form eines Differenzverstärkers, dem Balanced Ratiometric Detection System (BRD-System), möglich. In ersten Tests an einer Druckzelle konnte ein Zusammenhang zwischen der Stärke der Absorption und dem Luftdruck gezeigt werden. Zusätzlich wurden verschiedene Möglichkeiten zur Temperaturbestimmung eines Sauerstoffgases mit diesem Detektionssystem beschrieben.

Die Untersuchungen mit dem Kraftstoff-Detektionssystem am Serienmotor haben die Einsatzfähigkeit dieser Messtechnik bewiesen und gezeigt, dass zyklische Schwankungen in der Gemischbildung detektierbar sind und sich schon alleine mit der gemessenen Kraftstoffkonzentration Betriebszustände vergleichen lassen. Durch die Lichtmodulationsfrequenz von 5kHZ mit dem mechanischen Chopper kann die Kraftstoffkonzentration einzelner Motorzyklen allerdings nur bei niedriger Drehzahl $(<2000 \mathrm{U} / \mathrm{min})$ mit einer Auflösung von $1^{\circ} \mathrm{KW}$ bestimmt werden. Für eine Optimierung aktueller BDE-Motoren ist aber gerade die Kenntnis über die Gemischbildungsprozesse bei Drehzahlen > 3000U/min relevant, 
da bislang in diesen Betriebspunkten auf einen homogenen Betrieb umgestellt werden muss. Daher laufen derzeit Untersuchungen, die Halogenlampe und den mechanischen Chopper durch eine elektronisch bis zu einer Frequenz von $16 \mathrm{kHz}$ modulierbare IR-Diode zu ersetzen. Allerdings muss weiterhin ein Filter vor dem Detektor verwendet werden, da sonst die Hintergrundstrahlung zu groß ist. Um wirklich reale Gemischbildungsprozesse untersuchen zu können, ist aber ein gefeuerter Betrieb des Motors, also die Zündung des Luft/Kraftstoffgemisches, notwendig. Daher wird das Messsystem in diese Richtung weiterentwickelt. Erste Tests laufen bereits.

Das vorgestellte Messprinzip kann auch angewendet werden, um andere Molekülspezies zu detektieren. Beispielsweise ist es möglich unter Verwendung anderer Fasern und Filter $\mathrm{CO}_{2}$ im Brennraum zu detektieren $[22,23]$.

Für eine exakte Bestimmung des Lambda-Wertes wäre aber eine gleichzeitige Bestimmung der Sauerstoffkonzentration unerlässlich. Dafür bietet die Kombination der beiden, in diesem Kapitel vorgestellten Messtechniken eine sehr gute Möglichkeit. Allerdings sind aber andere schmalbandige Diodenlaser (z.B. VCSEL- oder DFB-Laser) in dem Wellenlängenbereich um 760nm derzeit schwer erhältlich und das vorgestellte Näherungsverfahren zur Lambda-Wert-Bestimmung ausschließlich aus der Kraftstoffkonzentration ist auf Grund der unbekannten lokalen Temperatur zu ungenau. Derzeit laufen daher Untersuchungen, die Sauerstoffkonzentration mit absorptionsspektroskopischen Messtechniken im ultravioletten Wellenlängenbereich zu bestimmen. 


\section{Literaturverzeichnis}

[1] Acker W.P., Serpengüzel A., Chang R.K. und Hill S.C.: Stimulated Raman Scattering of Fuel Droplets. Appl. Phys. B, 51:9-16, 1990.

[2] Adler, U.: Bosch - Automotive Handbook. VDI-Verlag GmbH, 3 Auflage, 1993.

[3] Agrawal, G.P.: Nonlinear Fiber Optics. Academic Press, New York, 1989.

[4] Allen M.G., Carleton K.L., Davis S.J. Kessler W.J. Otis C.E. PalomBO D.A. und Sonnenfroh D.M.: Ultrasensitive dual-beam absorption and gain spectroscopy: applications for near-infrared and visible diode laser sensors. Applied Optics, 34(18):3240-3249, 1995.

[5] Atkins, P.W.: Physikalische Chemie. VCH, 1996.

[6] Bünting U., Lewitzka F. und Karlitschek P.: Mathematical Model of a LaserInduced Fluorescence FiberOptic Sensor Head for Trace Detection of Pollutants in Soil. Appl. Spectrosc., 53(1):49-56, 1999.

[7] Borchers M.A., Esen C. und Schweiger G.: Cascade lasing with spherical microparticles. Opt. Lett., 26:346-348, 2001.

[8] Chen G., Mazumder Md.M., Chang R.K. Swindal J.C. und Acker W.P.: Laser Diagnostics for Droplet Characterization: Application of Morphology Dependent Resonances. Prog. Energy Combust. Sci., 22:163-188, 1996.

[9] Ching S.C., Lai H.M. und Young K.: Dielectric microspheres as optical cavities: Einstein $A$ and B coefficients and level shift. J.Opt.Soc.Am. B, 4(12):2004-2009, 1987.

[10] Ching S.C., Lai H.M. und Young K.: Dielectric microspheres as optical cavities: thermal spectrum and density of states. J.Opt.Soc.Am. B, 4(12):1995-2003, 1987.

[11] Cooney T.F., Skinner H.T. und Angel S.M.: Comparative Study of Some FiberOptic Remote Raman Probe Designs. Part I: Model for Liquids and Transparent Solids. Appl. Spectrosc., 50(7):836-848, 1996. 
[12] Dakin J., Culshaw B.: Optical Fiber Sensors. Artech House Publishers, 1997.

[13] DemtröDer, W.: Experimentalphysik 3. Springer-Verlag, 1996.

[14] D'Sa E.J., Lohrenz S.E.: Theoretical treatment of fluorescence detection by a dual-fiber-optic sensor with consideration of sampling variability and package effects associated with particles. Applied Optics, 38(12):2524-2534, 1999.

[15] Eckbreth, A.C.: Laser Diagnostics for Combustion Temperature and Species. Abacus Press, 1988.

[16] Esen C., Janetta F. und Borchers M.A.: Laseremission in sphärischen Mikropartikeln. LaserOpto, 32(6):41-46, 2000.

[17] Eversole J.D., Lin H.B., Huston A.L. Campillo A.J. Leung P.T. Liu S.Y. und Young K.: High-precision identification of morphology-dependent resonances in optical processes in microdroplets. J.Opt.Soc.Am. B, 10(10), 1993.

[18] Falbe, Regitz: RÖMPP Chemielexikon. Georg Thieme Verlag, 1995.

[19] Fujikawa T., Hattori Y. und Akinama K.: Quantitative 2-D Fuel Distribution Measurements in an S.I. Engine Using Laser-Induced Fluorescence with Suitable Combination of Fluorescence Tracer and Excitation Wavelength. SAE Technical Paper 972944, 1997.

[20] Ghandhi, J. B.: Single- and dual-fiber fluorescence probes: application to oil-film measurements in an internal combustion engine. Applied Optics, 39(30):5456-5464, 2000.

[21] GünZler, H. und HeISE H.M.: IR-Spektroskopie. VCH, Weinheim, 1996.

[22] Hall, M.J. und Zuzek P.: Fiber Optic Sensor for Time-Resolved Measurements of Exhaust Gas Recirculation in Engines. SAE Technical Paper 2000-01-2865, 2000.

[23] Hall M.J., Zuzek P. und Anderson R.W.: Fiber Optic Sensor for Crank Angle Resolved Measurements of Burned Gas Residual Fraction in the Cylinder of an SI Engine. SAE Technical Paper 2001-01-1921, 2001.

[24] HerzBerg, G.: Molecular Spectra and Molecular Structure, Band I: Spectra of Diatomic Molecules. Krieger Publishing Company, 1989.

[25] HerzBerg, G.: Molecular Spectra and Molecular Structure, Band II: Infrared and Raman Spectra of Polyatomic Molecules. Krieger Publishing Company, 1991.

[26] HobBs, PhiliP C.D.: Ultrasensitive laser measurements without tears. Applied Optics, 36(4):903-920, 1997.

[27] HobBs, PhiliP C.D.: Building Electro-Optical Systems: Making It All Work. WileyInterscience Publication, 2000. 
[28] Hosono H., Mizuguchi M., Skuja L.: Fluorine-doped $\mathrm{SiO}_{2}$ glasses for $\mathrm{F}_{2}$ excimer laser optics: fluorine content and color-center formation. Optics Letters, 24(22):15491551,1999 .

[29] Hultzsch, H.: Optische Telekommunikationssysteme. Damm-Verlag, Gelsenkirchen, 1996.

[30] Kannan S., Li J., Lehman R.L. Sigel G.H.: Excimer-laser-induced spatially variant luminescence in pure-silica core fibers with fluorine-doped silica cladding. Applied Optics, 32(33):6684-6689, 1993.

[31] Kazenwadel J., Koban W., Kunzelmann T. und Schulz C.: Fluorescence imaging of natural gas/air mixing without tracers added. Chemical Physics Letters, 345:259-264, 2001.

[32] Knapp M., Beushausen V., Hentschel W. Manz P. Grünefeld G. und AndResen P.: In-Cylinder Mixture Formation Analysis with Spontaneous Raman Scattering Applied to a Mass-Production SI Engine. SAE Technical Paper 970827, 1997.

[33] Koenig, M. und Hall M.J.: Measurements of Local In-Cylinder Fuel Concentration Fluctuations in a Firing SI Engine. SAE Technical Paper 971644, 1997.

[34] Koenig, M. und Hall M.J.: Cycle-Resolved Measurements of Pre-Combustion Fuel Concentration Near the Spark Plug in a Gasoline SI Engine. SAE Technical Paper 981053, 1998.

[35] Kornmesser, C.: Möglichkeiten und Grenzen planarer Lasermeßtechniken zur Analyse der Gemischbildung bei direkteinspritzenden Brennverfahren. Doktorarbeit, Universität Bielefeld, Cuvillier Verlag Göttingen, 2001.

[36] Lange S., Schweiger G.: Structural resonances in the total Ramanand fluorescence-scattering cross section: concentration-profile dependence. J.Opt.Soc.Am. B, 13(9):1864-1872, 1996.

[37] Lefebvre, A.: Atomisation and Spray. Taylor and Francis, first edition, 1989.

[38] Lenz, H.P.: Gemischbildung bei Ottomotoren. Springer-Verlag, 1990.

[39] Lin H.B., Huston A.L., Eversole J.D. Campillo A.J. und Chylek P.: Internal scattering effects on microdroplet resonant emission structure. Optics Letters, 17(14), 1992.

[40] MÜLLER, T.: Laserdiagnostische Verfahren zur Spray- und Gemischbildungsanalyse. Doktorarbeit, Universität Bielefeld, Cuvillier Verlag Göttingen, 2002.

[41] Müller T., Grünefeld G. und Beushausen V.: High-Precision Measurement of the Temperature of Methanol and Ethanol Droplets using Spontaneous Raman Scattering. Appl. Phys. B, 70:155-158, 2000. 
[42] Nishiyama A., Kawahara N. und Tomita E.: In-Situ Fuel Concentration Mea-

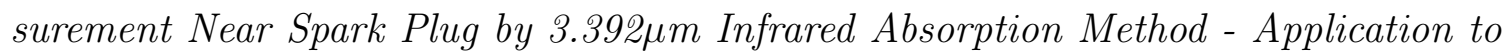
Spark Ignition Engine. SAE Technical Paper 2003-01-1109, 2003.

[43] Nishiyama A., Kawahara N., Tomita E. Fujiwara M. Ishikawa N. KaMei K. und NAGAShima K.: In-Situ Fuel Concentration Measurement Near Spark Plug by 3.392um Infrared Absorption Method - Application to a Port Injected LeanBurn Engine. SAE Technical Paper 2004-01-1353, 2004.

[44] Pinnick R.G., Biswas A., Pendelton J.D. und Armstrong R.L.: Aerosol Induced Laser Breakdown Thresholds: Effect of Resonant Particles. Applied Optics, 31:312-318, 1992.

[45] Pischinger R., Krassing G., Taucar G. und Sams Th.: Thermodynamik der Verbrennungskraftmaschine. Band 5, Springer-Verlag, 1989.

[46] REA, E.C. und R.K. HAnson: Rapid laser-wavelength modulation spectroscopy used as a fast temperature measurement technique in hydrocarbon combustion. Applied Optics, 27(21):4454-4464, 1988.

[47] Reboux J., Puechberty D. und Dionnet F.: A New Approach of Planar Laser Induced Fluorescence Applied to Fuel/Air Ratio Measurement in the Compression Stroke of an Optical S.I. Engine. SAE Technical Paper 941988, 1994.

[48] Reboux J., Puechberty D. und Dionnet F.: Study of Mixture Inhomogenities and Combustion Development in a S.I. Engine Using a New Approach of Laser Induced Fluorescence (FARLIF). SAE Technical Paper 961205, 1996.

[49] Rothman L.S., ET. AL.: The HITRAN Molecular Spectroscopic Database and HAWKS (HITRAN Atmospheric Workstation): 1996 edition.

[50] Schäfer, B.: Simulationsprogramm Pulsstrech. Optikgruppe - Laserlaboratorium Göttingen.

[51] Schmidt-Uhlig T., Kalitschek P., Marowsky G. und Sano Y.: New simplified coupling scheme for the delivery of $20 M W N d: Y A G$ laser pulses by large core optical fibers. Appl.Phys.B, 72:183-186, 2001.

[52] Schrader, B.: Infrared and Raman Spectroscopy. VCH, Weinheim, 1995.

[53] Schütte M., Grünefeld G., Nassif-Pugsley D. Andresen P. Hentschel W. und Homburg A.: Air/Fuel-Ratio Measurements in Direct-Injection Gasoline Sprays using 1-D Raman Scattering. SAE Technical Paper 2000-01-0244, 2000.

[54] Senior, J.M.: Optical Fiber Communications. Second Edition Prentice Hall, 1992.

[55] Serpengüzel A., Swindal J.C., Chang R.K. und Acker W.P.: Twodimensional imaging of sprays with fluorescence, lasing and stimulated Raman scattering. Applied Optics, 31(18):3543-3551, 1992. 
[56] Sharma A., Dokhanian M., Wu Z.Q. Posey R. Williams A. und VenkaTESWARLU P.: Stimulated Raman scattering in a multimode optical fiber with bendinduced loss. Optics Comm., 111:127-131, 1994.

[57] Shiraishi T., Nakayama Y., Nogi T. und Ohsuga M.: Effect of Spray Characteristics on Combustion in Direct Injection Spark Ignition Engine. SAE Technical Paper 980156, 1998.

[58] Sircar A., Dwivedi R.K. und Thareja R.K.: Laser induced Breakdown of Ar, $N$ and $O$-Gases using 1064, 532, 355, 266nm Radiation. Appl.Phys.B, 63:623-627, 1996.

[59] Tomita E., Kawahara N., Shigenaga M. Yoshiyama S. Hamamoto Y. KaKuho A. Itho T. und DibBle R.W.: An optical sensor instrumented in spark plug for in-situ fuel concentration measurement in an engine cylinder by $3.39 \mu m$ infrared absorption method. 11th International Symposium: Applications of Laser Techniques to Fluid Mechanics, July 2002, Lissabon, Portugal.

[60] Wang J., Sanders S.T., Jeffries J.B. Hanson R.K.: Oxygen measurements at high pressures with vertical cavity surface-emitting lasers. Appl. Phys. B, 2001.

[61] Warnatz J., Maas U. und Dibble R.W.: Verbrennung. Springer-Verlag, 1997.

[62] Wolfrum J., Dreier T., Ebert V. und Schulz C.: Laser-based Combustion Diagnostics, in Encyclopedia of Analytical Chemistry, pp.2118-2148. John Wiley \& Sons Ltd, Chichester, 2000.

[63] Zhao H., Ladommatos N.: Optical Diagnostics for In-Cylinder Mixture Formation Measurements in IC Engines. Prog. Energy Combust. Sci., 24:297-336, 1998.

[64] Zhao H., Ladommatos N.: Engine Combustion Instrumentation and Diagnostics. Society of Automotive Engineers, 2001. 


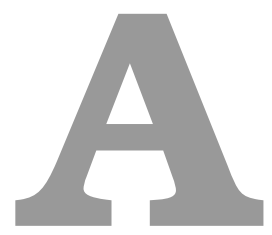

Anhang 


\section{Balanced Ratiometric Detection System (Bauteile)}

\begin{tabular}{|l|l|l|l|}
\hline Parts in fig & Device & Type no. & Manufacturer \\
\hline $\mathrm{A}_{1}, \mathrm{~A}_{2}$ & Operational amplifier & OP-470EY & Analog devices \\
\hline $\mathrm{Q}_{1}, \mathrm{Q}_{2}, \mathrm{Q}_{\mathrm{s}}, \mathrm{Q}_{\mathrm{h}}$ & NPN-bipolar transistor & MAT-04FP & Analog devices \\
\hline $\mathrm{Q}_{3}$ & PNP-darlington bipolar transistor & MPSA-64 & Motorola \\
\hline $\mathrm{D}_{1}, \mathrm{D}_{2}$ & InGaAs-photodiode & FGA10 & Thorlabs \\
\hline $\mathrm{Z}_{\mathrm{s}}$ & Precision reference (zener diode) & LM399 & National semiconductor \\
\hline $\mathrm{Q}_{4}$ & NPN-darlington bipolar transistor & MPSA14 & Motorola \\
\hline $\mathrm{B}$ & High speed buffer & LM6321 & National semiconductor \\
\hline Not shown in Fig & Voltage regulator & $\begin{array}{l}\text { LM317, } \\
\text { LM137 }\end{array}$ & National semiconductor \\
\hline
\end{tabular}




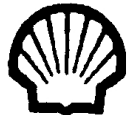

Deutsche Shell GmbH

PAE - Labor, OGMF/I

18. Januar 2002

Hohe-Schaar-Str. 36

21107 Hamburg

\section{Prüfbericht 02/X000060/1}

$\begin{array}{ll}\text { Prufgegenstand } & : \text { PR1632 } \\ \text { Licferstclle } & : \\ \text { Name d. Kunden } & : \\ \text { Probenalime am } & : 27.12 .01 \\ \text { Probenalime aus } & : \text { Faß } \\ \text { Probenahme von } & : \text { Sk } \\ \text { Laboreingang } & : 14.01 .02 \\ \text { Laborausgang } & : 18.01 .02\end{array}$

Auftraggeber : LaserLabor Göttingen

StraDe PLZ / Ort

Telefon

Fax-Nr. Weitere luformationen zum Prufgegenstand (z.B. Zustand): 40 L farbloser Kraftstoff fur Laser Labor in Göttingen,
Herrn Dr. Beushausen

Grund der Untersuchung:

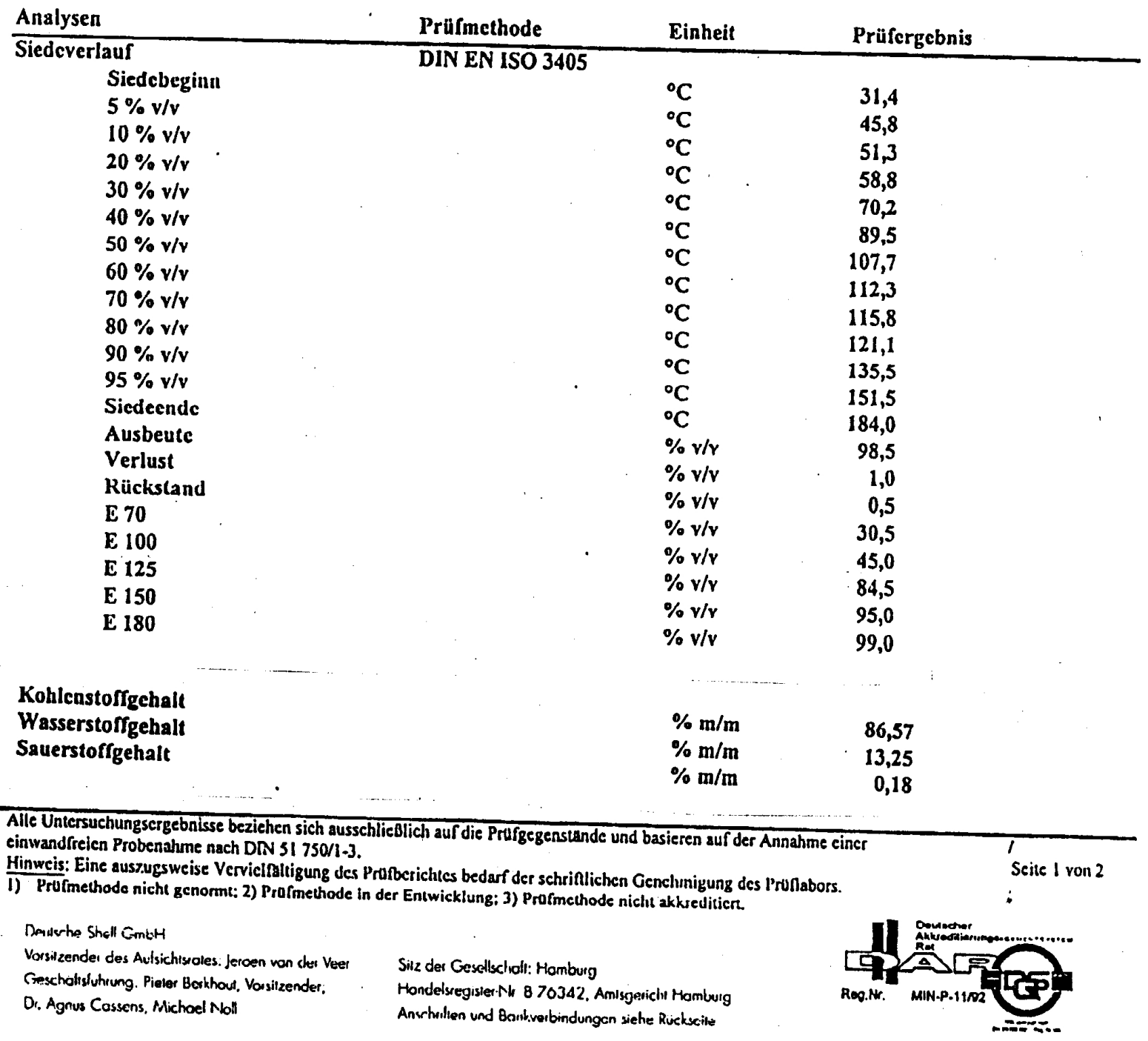


Tab. A1: Brennwerte und Elementaranalysedaten handelsüblicher Kraftstoffe *

\begin{tabular}{|c|c|c|c|c|c|c|c|c|}
\hline \multirow[t]{2}{*}{ Nr. } & \multirow[t]{2}{*}{ Sorte } & \multirow{2}{*}{$\begin{array}{c}\text { Dichte } 15^{\circ} \mathrm{C} \\
\mathrm{kg} / \mathrm{m}^{3}\end{array}$} & \multicolumn{3}{|c|}{ Elementaranalyse } & Ho & \multicolumn{2}{|c|}{$\mathrm{Hu}$} \\
\hline & & & $\mathrm{C}[\mathrm{m} \%]$ & $\mathrm{H}[\mathrm{m} \%]$ & $O[\mathrm{~m} \%]$ & $\mathrm{MJ} / \mathrm{kg}$ & $\mathrm{MJ} / \mathrm{kg}$ & $\mathrm{MJ} / \mathrm{Itr}$ \\
\hline 1 & Normal bleifrei & 730,6 & 86,85 & 12,51 & 0,23 & 44,62 & 41,89 & 30,61 \\
\hline \multirow[t]{2}{*}{3} & $"$ & 735,6 & 87,75 & 11,94 & 0,12 & 44,09 & 41,49 & 30,52 \\
\hline & Mittelwert & 735,1 & 87,47 & 12,14 & 0,13 & 44,17 & 41,52 & 30,52 \\
\hline 5 & " & 755,3 & 88,65 & 11,04 & 0,36 & 43,10 & 40,69 & 30,73 \\
\hline 6 & " & 746,7 & 88,65 & 11,40 & 0,04 & 43,42 & 40,93 & 30,56 \\
\hline \multirow[t]{2}{*}{7} & " & 756,9 & 88,06 & 11,32 & 0,44 & 44,11 & 41,64 & 31,52 \\
\hline & Mittelwert & 751,0 & 88,40 & 11,28 & 0,24 & 43,44 & 40,98 & 30,78 \\
\hline 8 & Super Plus bleifrei & 753,1 & 87,16 & 11,40 & 1,47 & 42,80 & 40,31 & 30,36 \\
\hline 11 & Mittelwert & 761,0 & 87,63 & 10,94 & 1,33 & 42,69 & 40,31 & 30,67 \\
\hline 12 & Dieselkraftstoff & 829,8 & 86,32 & 13,18 & - & 45,74 & 42,87 & 35,57 \\
\hline 13 & $"$ & 837,1 & 85,59 & 12,70 & - & 45,67 & 42,90 & 35,91 \\
\hline \multirow[t]{2}{*}{14} & $"$ & 828,3 & 86,05 & 13,70 & - & 46,11 & 43,12 & 35,72 \\
\hline & Mittelwert & 831,7 & 85,99 & 13,19 & - & 45,84 & 42,96 & 35,73 \\
\hline
\end{tabular}

* Die Zusammensetzung der untersuchten Kraftstoffe ist in Tab. A2 aufgeführt Quelle: DGMK, Hamburg

Tab. A2: Zusammensetzun der in Tab. A1 aufgeführten Kraftstoffe

\begin{tabular}{|c|c|c|c|c|c|c|c|}
\hline \multirow[t]{2}{*}{ Nr. } & \multirow[t]{2}{*}{ Sorte } & \multicolumn{3}{|c|}{ FIA - Analyse [ Vol \% ] } & \multirow{2}{*}{$\frac{\text { GC - Aromaten }}{[\mathrm{M} \%]}$} & \multirow{2}{*}{$\frac{\text { MTBE }}{[\mathrm{Vol} \%]}$} & \multirow{2}{*}{$\begin{array}{l}\mathrm{HPLC} \text { * } \\
{[\mathrm{M} \%]}\end{array}$} \\
\hline & & Aromaten & Olefine & Parafine & & & \\
\hline 1 & Normal bleifrei & 24,5 & 20,5 & 55 & 28,4 & - & - \\
\hline 2 & " & 32 & 19,5 & 48,5 & 36,2 & - & - \\
\hline 3 & $"$ & 29,5 & 23 & 47,5 & 33,9 & - & - \\
\hline 4 & Super bleifrei & 34 & 14,6 & 48,5 & 38,8 & - & - \\
\hline 5 & " & 41 & 8,3 & 48,4 & 47,8 & - & - \\
\hline 6 & $"$ & 36 & 12 & 52 & 40,3 & - & - \\
\hline 7 & $"$ & - & - & - & 43,3 & - & - \\
\hline 8 & Suber Plus bleifrei & 39,9 & 5,9 & 44,9 & 48,3 & 9,3 & - \\
\hline 9 & " & 51,6 & 5,1 & 35,5 & 56,8 & 7,7 & - \\
\hline 10 & $"$ & 38,2 & 7,4 & 47,5 & 43,3 & 6,8 & - \\
\hline 11 & $"$ & - & - & - & 56 & 9,8 & - \\
\hline 12 & Dieselkraftstoff & - & - & - & - & - & 23,9 \\
\hline 13 & " & - & - & - & - & - & 7,5 \\
\hline 14 & $"$ & - & - & - & - & - & 20,4 \\
\hline
\end{tabular}

* Hochdruckflüssigkeitschromatographie

Quelle: DGMK, Hamburg 
Tab. 33: Gemischneizwerte von Testkraftstoffen

\begin{tabular}{|c|c|c|c|c|c|c|c|c|}
\hline \multicolumn{2}{|l|}{ Krattstott- Nr. : } & \multirow{2}{*}{$\begin{array}{c}\text { Dichio } \\
\text { bai } \\
15^{-0} \mathrm{C} \\
0 / \mathrm{mi} \\
\end{array}$} & \multirow{2}{*}{$\begin{array}{l}\text { stoch. } \\
\text { Luttbedant } \\
\mathrm{kg} / \mathrm{mol}\end{array}$} & \multirow{2}{*}{$\begin{array}{l}\text { stocti. } \\
\text { Gamisch- } \\
\text { nelzwen } \\
\text { kJ/com }\end{array}$} & \multirow{2}{*}{$\begin{array}{c}\text { Molekutat - } \\
\text { Gewicht } \\
\text { a } / \mathrm{mgl}\end{array}$} & \multicolumn{3}{|c|}{ Elementaranalyse } \\
\hline & i & & & & & $\begin{array}{c}\mathrm{C} \\
\mathrm{Mol} / \mathrm{ko}\end{array}$ & $\begin{array}{c}\mathrm{H} \\
\text { Mol / ko }\end{array}$ & $\begin{array}{c}0 \\
\mathrm{Mol} / \mathrm{kg}\end{array}$ \\
\hline 1 & & 0.771 & 14.12 & - & $1 . \quad 1$ & 720 & 121.8 & 0.86 \\
\hline 2 & & 0.771 & 14.12 & - & - & 720 & 121.8 & 0.86 \\
\hline$\Xi$ & & 0.692 & 15.12 & 3485 & 114.2 & 70.2 & 158.0 & 0 \\
\hline 1 & & 0.879 & 13.27 & 3543 & 78.1 & 76.6 & 77.0 & 0 \\
\hline 5 & & 0.867 & 13.50 & 3532 & 921 & 76.0 & 88.0 & 0 \\
\hline 6 & & 0.862 & 13.67 & 3499 & 106.2 & 75.3 & 94.2 & 0 \\
\hline 7 & & 0.790 & 6.47 & 3234 & 32.9 & 31.1 & 125.0 & 39 \\
\hline 8 & $!$ & 0.695 & 15.08 & 3487 & 113.1 & 70.3 & 156.2 & 0 \\
\hline 9 & $i$ & 0.703 & 14.99 & 3488 & 110.6 & 70.6 & 1522 & 0 \\
\hline 10 & j & 0.714 & 14.85 & 3483 & 107.0 & 71.0 & 144.8 & 0 \\
\hline 11 & $!$ & 0.698 & 15.05 & 3488 & 113.1 & 70.4 & 155.0 & 0 \\
\hline 12 & $\vdots$ & 0.704 & 14.99 & 3488 & 1120 & 70.5 & 151.8 & 0 \\
\hline 13 & $!$ & 0.710 & 14.92 & 3490 & 110.9 & 70.9 & 149.1 & 0 \\
\hline 14 & 1 & 0.796 & 14.85 & 3482 & 109.8 & 71.0 & 145.8 & 0 \\
\hline 15 & ' & 0.698 & 15.05 & 3486 & 113.8 & 70.4 & 154.9 & 0 \\
\hline 16 & $i$ & 0.705 & 14.98 & 3487 & 113.4 & 70.5 & 1151.8 & 0 \\
\hline 17 & 1 & 0.712 & 14.92 & 3487 & 113.0 & 70.9 & 148.9 & 0 \\
\hline 18 & $!$ & 0.719 & 14.85 & 3487 & 112.6 & 71.0 & 145.8 & 0 \\
\hline 19 & & 0.700 & 15.05 & 3487 & 113.6 & 70.4 & 154.9 & 0 \\
\hline 20. & 1 & 0.705 & 14.98 & 3489 & 113.4 & 70.6 & 1520 & 0 \\
\hline 21 & 1 & 0.712 & 14.92 & 3481 & 113.0 & 70.8 & 148.8 & 0 \\
\hline 22 & $i$ & 0.720 & 14.85 & 3502 & 112.6 & 71.0 & 145.8 & 0 \\
\hline 23 & 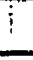 & 0.631 & 15.09 & 3492 & 111.2 & 70.3 & 156.8 & 0 \\
\hline 24 & 1 & 0.683 & 14,58 & - & 100.4 & 68.4 & 156.2 & 1.78 \\
\hline 25 & $\therefore$ & 0.704 & 13.59 & 3475 & 76.6 & 61,4 & 162.9 & 6.20 \\
\hline 26 & 1 & 0.731 & 14.67 & 3507 & 106.0 & 71.8 & 138.0 & 0 \\
\hline 27 & $i$ & 0.738 & 14.59 & 3510 & 104.2 & 72.1 & 134.6 & 0 \\
\hline 28 & 1 & $0.72 \mathrm{a}$ & 14.67 & 3507 & 106.0 & 71.8 & 138.0 & 0 \\
\hline 29 & 1 & 0.738 & 14.59 & 3510 & 104.2 & 72.1 & 134.6 & 0 \\
\hline 30 & $:$ & 0.749 & 14.06 & 3468 & 91.6 & 70.2 & 128.9 & 1.62 \\
\hline
\end{tabular}

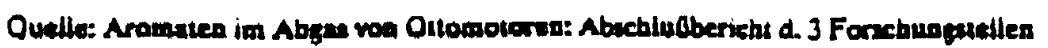

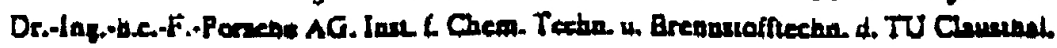




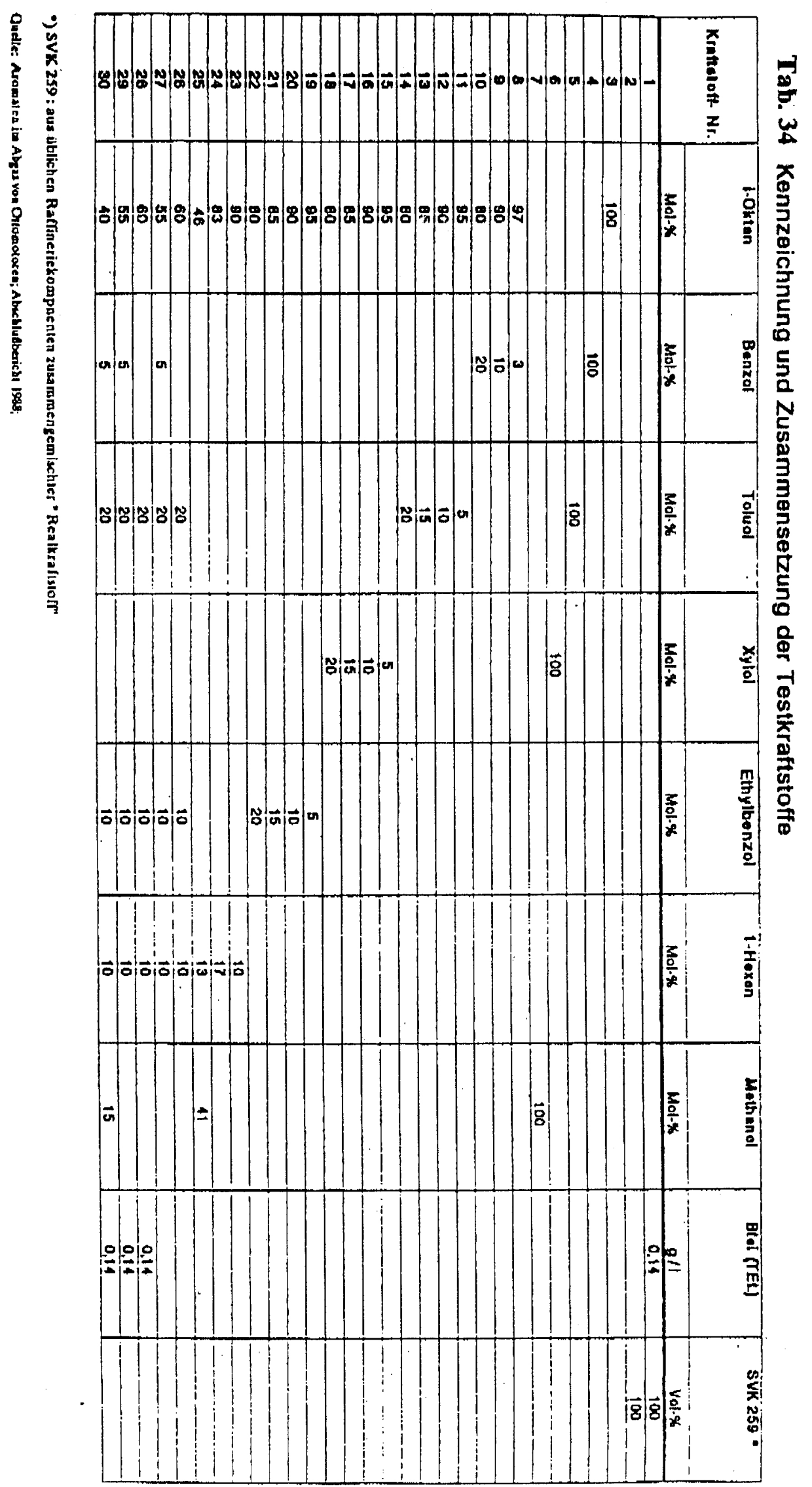




\section{Lebenslauf}

\section{Persönliche Angaben}

Nachname:

Thiele

Vorname

Olaf

Addresse:

Holbornweg 2

Geburtstag: 37077 Göttingen

Geburtsort: 20.03.1973

Familienstand:

Hannover

Staatsangehörigkeit: verheiratet deutsch

\section{Schulbildung}

$$
\begin{aligned}
& 1979-1983 \\
& 1983-1985 \\
& 1985-1992 \\
& 15.5 .1992
\end{aligned}
$$

\section{Zivildienst}

1.7.1992 - 30.9.1993

\section{Studium}

WS 93/94

5.10 .1995

Juli '98 - Juli '99

September 1999

Seit November 1999
Grundschule Grimsehlweg

Orientierungsstufe Gartenheimstraße

Leibniz-Gymnasium

Allgemeine Hochschulreife am Leibniz-Gymnasium

Landesbildungszentrum für Blinde in Hannover

Beginn des Studiums der Physik an der Universität Hannover

Vordiplom in Physik

Diplomarbeit am Laser Zentrum Hannover e.V. Diplom in Physik an der Universität Hannover Promotion in Physik am Laser-Laboratorium Göttingen e.V. 